Supporting Information

\title{
Bromine Radical Catalysis by Energy Transfer Photosensitization
}

\author{
Dian-Feng Chen, Cameron Chrisman, and Garret M. Miyake* \\ Department of Chemistry, Colorado State University, Ft Collins, Colorado 80523, United \\ States
}

\section{Table of Contents}

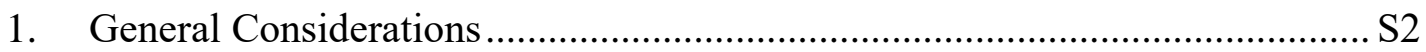

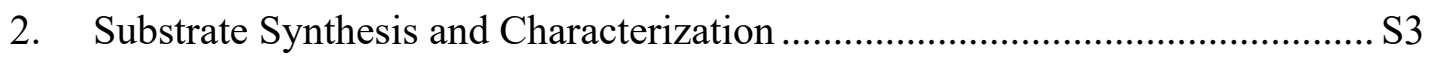

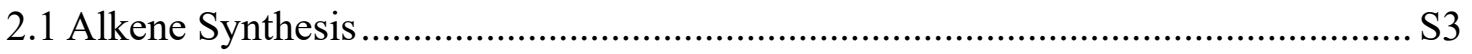

2.2 Vinylcyclopropane Synthesis ........................................................................... S5

3. Complementary Optimization of Conditions.................................................... S8

4. General Reaction Setup and Experimental Procedures ................................... S11

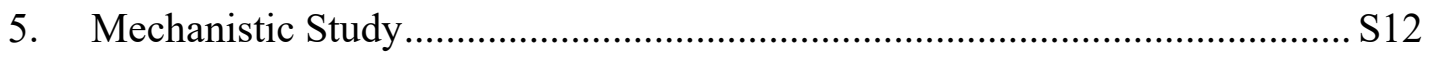

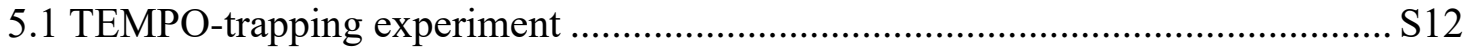

5.2 Investigation of alternative precatalysts............................................................... S13

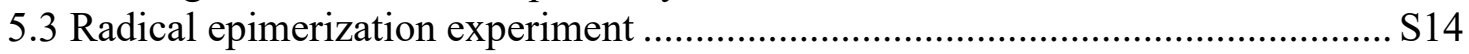

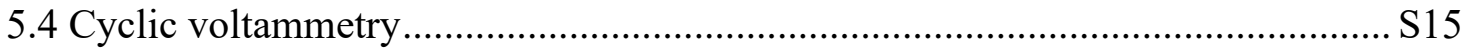

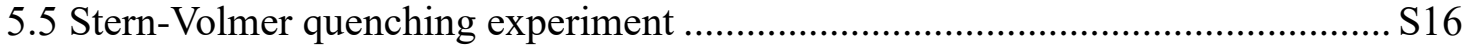

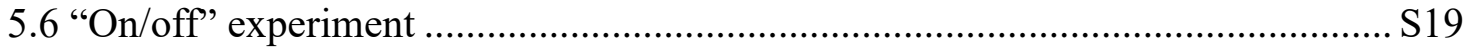

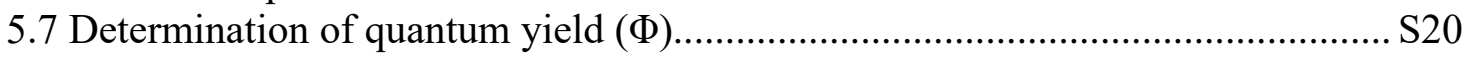

6. Characterization of Products.......................................................................... S22

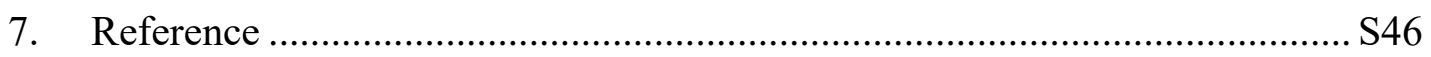

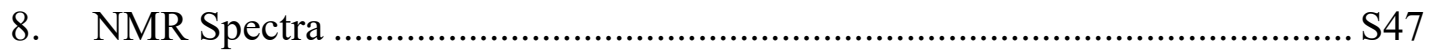




\section{General Considerations}

Commercial reagents and anhydrous solvents were purchased from Millipore-Sigma, Alfa and TCI, and were used as received unless otherwise indicated. All catalytic reactions were carried out under $\mathrm{N}_{2}$ with oven-dried vials. Thin layer chromatography was performed on SiliCycle ${ }^{\circledR} 250$ um, 60A plates. Column chromatography was performed on SiliCycle ${ }^{\circledR}$ SilicaFlash ${ }^{\circledR}$ P60, 40-63 um, 60A. Visualization was accomplished with I2-silica.

${ }^{1} \mathrm{H},{ }^{13} \mathrm{C}$, and ${ }^{19} \mathrm{~F}$ NMR spectra were recorded on a Bruker $400 \mathrm{~Hz}(101 \mathrm{~Hz}, 162 \mathrm{~Hz}$ and $376 \mathrm{~Hz}$ for ${ }^{13} \mathrm{C},{ }^{31} \mathrm{P}$ and ${ }^{19} \mathrm{~F}$, respectively) spectrometer at ambient temperature. All NMR spectra are referenced to the residual solvent $\left(\mathrm{CHCl}_{3}\right)$ signal. Data for ${ }^{1} \mathrm{H},{ }^{19} \mathrm{~F}$ and ${ }^{31} \mathrm{P} \mathrm{NMR}$ are reported as follows: chemical shift $(\delta \mathrm{ppm})$, multiplicity $(\mathrm{s}=$ singlet, $\mathrm{d}=$ doublet, $\mathrm{t}=$ triplet, $\mathrm{q}=$ quartet, $\mathrm{m}=$ multiplet, $\mathrm{br}=$ broad), coupling constant $(\mathrm{Hz})$, integration. Data for ${ }^{13} \mathrm{C}$ NMR are reported as follows: chemical shift ( $\left.\delta \mathrm{ppm}\right)$.

Mass spectra (MS) were obtained from Colorado State University Central Instrument Facility on an Agilent 6224 Time of flight (TOF) LC-MS spectrometer using ESI+/DART+ ionization model. 


\section{Substrate Synthesis and Characterization}

\subsection{Alkene Synthesis}
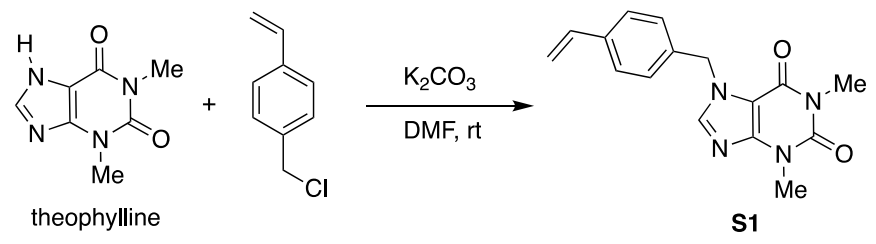

To a solution of theophylline $(0.90 \mathrm{~g}, 5.0 \mathrm{mmol}, 1.0$ equiv) in $15.0 \mathrm{~mL}$ of anhydrous DMF, 4-vinylbenzyl chloride (1.15 g, 7.5 mmol, 1.5 equiv) and $\mathrm{K}_{2} \mathrm{CO}_{3}$ (1.38g, 10.0 mmol, 2.0 equiv) were added sequentially. The slurry was vigorously stirred at room temperature for $12 \mathrm{~h}$. The white solid was filtered off, and the filtrate was diluted with EtOAc $(100 \mathrm{~mL})$, washed with $\mathrm{H}_{2} \mathrm{O}(50 \mathrm{~mL} \times 3)$, brine $(50 \mathrm{~mL})$, dried over anhydrous $\mathrm{Na}_{2} \mathrm{SO}_{4}$, and concentrated in vасио. The residue was subjected to silica gel chromatography using hexane/acetone (10:1 to 5:1) as the eluent, giving $\mathbf{S 1}$ as a white solid (1.31g, 89\% yield). ${ }^{1} \mathbf{H}$ NMR $\left(400 \mathrm{MHz}, \mathrm{CDCl}_{3}\right) \delta 7.56(\mathrm{~s}, 1 \mathrm{H}), 7.46-7.35(\mathrm{~m}, 2 \mathrm{H}), 7.30-7.26(\mathrm{~m}, 2 \mathrm{H})$, $6.68(\mathrm{dd}, J=17.6,10.9 \mathrm{~Hz}, 1 \mathrm{H}), 5.74(\mathrm{dd}, J=17.6,0.8 \mathrm{~Hz}, 1 \mathrm{H}), 5.48(\mathrm{~s}, 2 \mathrm{H}), 5.27(\mathrm{dd}, J$ $=10.9,0.8 \mathrm{~Hz}, 1 \mathrm{H}), 3.58(\mathrm{~s}, 3 \mathrm{H}), 3.40(\mathrm{~s}, 3 \mathrm{H}) .{ }^{13} \mathbf{C} \mathbf{N M R}\left(101 \mathrm{MHz}, \mathrm{CDCl}_{3}\right) \delta 155.3$, 151.7, 148.9, 140.8, 138.0, 136.0, 134.7, 128.2, 126.9, 114.8, 107.0, 50.1, 29.8, 28.0. DART-TOF-LC/MS m/z calcd. for $\mathrm{C}_{16} \mathrm{H}_{17} \mathrm{~N}_{4} \mathrm{O}_{2}\left[\mathrm{M}+\mathrm{H}^{+}\right]$297.1352, found 297.1351.

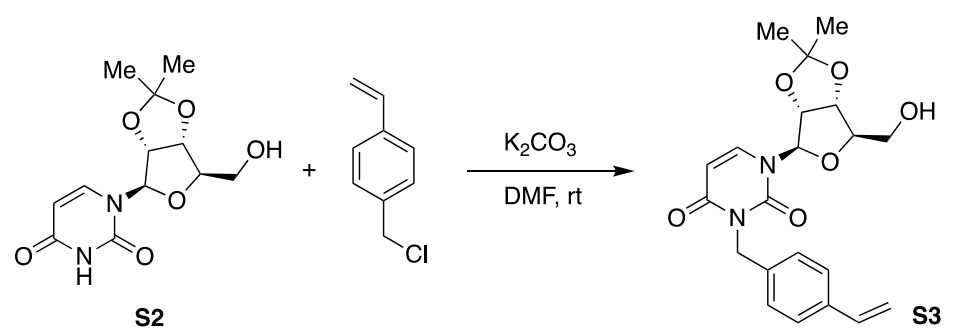

To a solution of protected uridine $\mathbf{S 2}^{1}$ (1.42 g, $5.0 \mathrm{mmol}, 1.0$ equiv) in $15.0 \mathrm{~mL}$ of anhydrous DMF, 4-vinylbenzyl chloride $(1.15 \mathrm{~g}, 7.5 \mathrm{mmol}, 1.5 \mathrm{gequiv})$ and $\mathrm{K}_{2} \mathrm{CO}_{3}(1.38 \mathrm{~g}$, $10.0 \mathrm{mmol}, 2.0$ equiv) were added sequentially. The slurry was vigorously stirred at room temperature. After $12 \mathrm{~h}$, the solid was filtered off. The filtrate was diluted with EtOAc (100 $\mathrm{mL})$, washed with $\mathrm{H}_{2} \mathrm{O}(50 \mathrm{~mL} \times 3)$, brine $(50 \mathrm{~mL})$, dried over anhydrous $\mathrm{Na}_{2} \mathrm{SO}_{4}$, and concentrated in vacuo. The residue was subjected to silica gel chromatography using hexane/acetone (10:1 to 5:1) as the eluent, giving $\mathbf{S 3}$ as a colorless oil (1.41g, $78 \%$ yield). ${ }^{1} \mathbf{H}$ NMR $\left(400 \mathrm{MHz}, \mathrm{CDCl}_{3}\right) \delta 7.37(\mathrm{~d}, J=8.2 \mathrm{~Hz}, 2 \mathrm{H}), 7.29(\mathrm{~d}, J=8.3 \mathrm{~Hz}, 2 \mathrm{H}), 7.22(\mathrm{~d}$, $J=13.3 \mathrm{~Hz}, 1 \mathrm{H}), 6.63(\mathrm{dd}, J=17.6,10.9 \mathrm{~Hz}, 1 \mathrm{H}), 5.73(\mathrm{~d}, J=8.0 \mathrm{~Hz}, 1 \mathrm{H}), 5.66(\mathrm{dd}, J=$ 17.6, $1.0 \mathrm{~Hz}, 1 \mathrm{H}), 5.48$ (d, $J=2.9 \mathrm{~Hz}, 1 \mathrm{H}), 5.17(\mathrm{dd}, J=10.9,0.9 \mathrm{~Hz}, 1 \mathrm{H}), 5.08-4.88$ $(\mathrm{m}, 4 \mathrm{H}), 4.23(\mathrm{q}, J=3.1 \mathrm{~Hz}, 1 \mathrm{H}), 3.89-3.81(\mathrm{~m}, 1 \mathrm{H}), 3.74(\mathrm{ddd}, J=12.0,7.5,3.3 \mathrm{~Hz}$, $1 \mathrm{H}), 2.70(\mathrm{dd}, J=7.5,3.3 \mathrm{~Hz}, 1 \mathrm{H}), 1.52(\mathrm{~s}, 3 \mathrm{H}), 1.30(\mathrm{~s}, 3 \mathrm{H}) .{ }^{13} \mathbf{C}$ NMR $(101 \mathrm{MHz}$, $\left.\mathrm{CDCl}_{3}\right) \delta 162.4,151.0,140.7,137.1,136.4,135.9,129.5,126.2,114.3,114.0,102.2,97.0$, 86.9, 83.8, 80.3, 62.7, 43.9, 27.3, 25.3. ESI-TOF-LC/MS m/z calcd. for $\mathrm{C}_{21} \mathrm{H}_{25} \mathrm{~N}_{2} \mathrm{O}_{6}$ $\left[\mathrm{M}+\mathrm{H}^{+}\right]$401.1713, found 401.1710. 


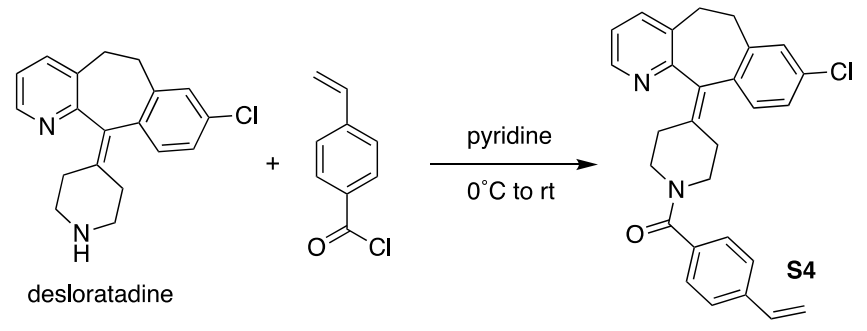

To a solution of desloratadine $(1.0 \mathrm{~g}, 3.2 \mathrm{mmol}, 1.0$ equiv) in $5.0 \mathrm{~mL}$ of anhydrous pyridine at $0{ }^{\circ} \mathrm{C}$, 4-vinylbenzoyl chloride $(0.54 \mathrm{~g}, 3.2 \mathrm{mmol}, 1.0$ equiv) was added dropwise. The mixture was stirred at $0{ }^{\circ} \mathrm{C}$ for $30 \mathrm{~min}$, then was warmed up to room temperature and stirred for $12 \mathrm{~h}$. The reaction was quenched by addition of sat. $\mathrm{NH}_{4} \mathrm{Cl}(20 \mathrm{~mL})$, then diluted with $\mathrm{CH}_{2} \mathrm{Cl}_{2}(100 \mathrm{~mL})$. The layers were separated, and the aqueous layer was extracted with $\mathrm{CH}_{2} \mathrm{Cl}_{2}(10 \mathrm{~mL} \times 2)$. The combined organic phase was washed with $\mathrm{H}_{2} \mathrm{O}(50 \mathrm{~mL} \times 3)$, brine $(50 \mathrm{~mL})$, dried over anhydrous $\mathrm{Na}_{2} \mathrm{SO}_{4}$, and concentrated in vacuo. The residue was subjected to silica gel chromatography using hexane/acetone (5:1 to 3:1) as the eluent, giving the final product as a while solid $(1.12 \mathrm{~g}, 79 \%$ yield $) .{ }^{1} \mathbf{H} \mathbf{~ N M R}\left(400 \mathrm{MHz}, \mathrm{CDCl}_{3}\right) \delta$ 8.38 (brs, 1H), $7.48-7.33(\mathrm{~m}, 5 \mathrm{H}), 7.22-7.01(\mathrm{~m}, 4 \mathrm{H}), 6.70(\mathrm{dd}, J=17.6,10.9 \mathrm{~Hz}, 1 \mathrm{H})$, $5.77(\mathrm{dd}, J=17.6,0.8 \mathrm{~Hz}, 1 \mathrm{H}), 5.29(\mathrm{~J}=10.9,0.8 \mathrm{~Hz}, 1 \mathrm{H}), 4.18$ (brs, $1 \mathrm{H}), 3.64$ (brs, $1 \mathrm{H})$, $3.45-3.13(\mathrm{~m}, 4 \mathrm{H}), 2.92-2.73(\mathrm{~m}, 4 \mathrm{H}), 2.70-2.15(\mathrm{~m}, 4 \mathrm{H}) .{ }^{13} \mathbf{C}$ NMR $(101 \mathrm{MHz}$, $\left.\mathrm{CDCl}_{3}\right) \delta 170.2,156.8,146.7,139.6,138.9,137.6,136.9,136.1,135.2,134.7,133.4,133.0$, 130.5, 129.0, 127.3, 126.2, 122.4, 115.2, 31.7, 31.5. ESI-TOF-LC/MS m/z calcd. for $\mathrm{C}_{28} \mathrm{H}_{26} \mathrm{ClN}_{2} \mathrm{O}\left[\mathrm{M}+\mathrm{H}^{+}\right] 441.1734$, found 441.1735 .

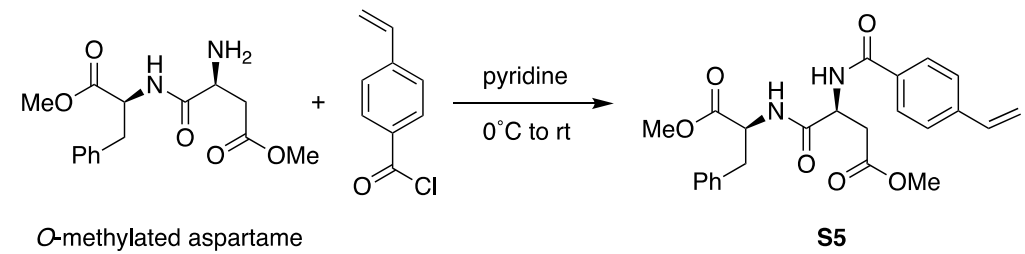

To a solution of O-methylated aspartame ${ }^{2}(0.72 \mathrm{~g}, 2.0 \mathrm{mmol}, 1.0$ equiv) in $4.0 \mathrm{~mL}$ of anhydrous pyridine at $0{ }^{\circ} \mathrm{C}$, 4-vinylbenzoyl chloride $(0.33 \mathrm{~g}, 2.0 \mathrm{mmol}, 1.0$ equiv) was added dropwise. The mixture was stirred at $0{ }^{\circ} \mathrm{C}$ for $30 \mathrm{~min}$, then was warmed up to room temperature and stirred for $12 \mathrm{~h}$. The layers were separated, and the aqueous layer was extracted with $\mathrm{CH}_{2} \mathrm{Cl}_{2}(10 \mathrm{~mL} \times 2)$. The combined organic phase was washed with $\mathrm{H}_{2} \mathrm{O}$ $(50 \mathrm{~mL} \times 3)$, brine $(50 \mathrm{~mL})$, dried over anhydrous $\mathrm{Na}_{2} \mathrm{SO}_{4}$, and concentrated in vacuo. The residue was subjected to silica gel chromatography using $\mathrm{CH}_{2} \mathrm{Cl}_{2} / \mathrm{MeOH}$ (20:1 to 1:1) as the eluent, giving the final product as a colorless oil $\left(0.73 \mathrm{~g}, 83 \%\right.$ yield). ${ }^{1} \mathbf{H}$ NMR (400 $\left.\mathrm{MHz}, \mathrm{CDCl}_{3}\right) \delta 7.70(\mathrm{~d}, J=8.3 \mathrm{~Hz}, 2 \mathrm{H}), 7.64(\mathrm{~d}, J=8.0 \mathrm{~Hz}, 1 \mathrm{H}), 7.48(\mathrm{~d}, J=8.3 \mathrm{~Hz}$, $2 \mathrm{H}), 7.18-7.02(\mathrm{~m}, 6 \mathrm{H}), 6.76(\mathrm{dd}, J=17.6,10.9 \mathrm{~Hz}, 1 \mathrm{H}), 5.87(\mathrm{~d}, J=17.5 \mathrm{~Hz}, 1 \mathrm{H})$, $5.39(\mathrm{~d}, J=10.9 \mathrm{~Hz}, 1 \mathrm{H}), 5.00(\mathrm{ddd}, J=7.9,6.4,3.8 \mathrm{~Hz}, 1 \mathrm{H}), 4.80(\mathrm{td}, J=7.3,5.3 \mathrm{~Hz}$, $1 \mathrm{H}), 3.73(\mathrm{~s}, 3 \mathrm{H}), 3.72(\mathrm{~s}, 3 \mathrm{H}), 3.22-2.98(\mathrm{~m}, 3 \mathrm{H}), 2.67(\mathrm{dd}, J=17.0,6.4 \mathrm{~Hz}, 1 \mathrm{H}) .{ }^{13} \mathrm{C}$ NMR $\left(101 \mathrm{MHz}, \mathrm{CDCl}_{3}\right) \delta 173.1,171.4,170.1,166.7,141.2,135.9,135.6,132.2,129.2$, $128.5,127.5,127.1,126.4,116.3,53.5,52.4,52.2,49.3,37.7,34.9$. DART-TOF-LC/MS $\mathrm{m} / \mathrm{z}$ calcd. for $\mathrm{C}_{24} \mathrm{H}_{30} \mathrm{~N}_{3} \mathrm{O}_{6}\left[\mathrm{M}+\mathrm{NH}_{4}{ }^{+}\right]$456.2135, found 456.2133 . 


\subsection{Vinylcyclopropane Synthesis}

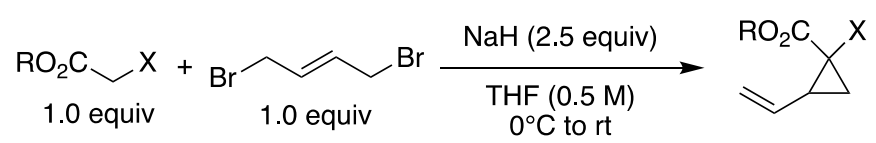

Procedure A: To a suspension of $60 \% \mathrm{NaH}(1.0 \mathrm{~g}, 25.0 \mathrm{mmol}, 2.5 \mathrm{equiv})$ in $25.0 \mathrm{~mL}$ of anhydrous THF at $0{ }^{\circ} \mathrm{C}$, trans-1,4-dibromo-2-butene ( $2.1 \mathrm{~g}, 10.0 \mathrm{mmol}, 1.0$ equiv) was added in one portion. Appropriate activated methylene compounds ( $10.0 \mathrm{mmol}, 1.0$ equiv) was added dropwise over $10 \mathrm{~min}$. The reaction was then vigorously stirred at room temperature. Conversion was assessed by TLC (10\% EtOAc in hexanes). Upon completion, the reaction was cooled to $0{ }^{\circ} \mathrm{C}$ with an ice bath and carefully quenched by slow addition of sat. $\mathrm{NH}_{4} \mathrm{Cl}(10 \mathrm{~mL})$. The mixture was then diluted with $\mathrm{H}_{2} \mathrm{O}(30 \mathrm{~mL})$ and ether $(100 \mathrm{~mL})$. The layers were separated, and the aqueous phase was extracted with ether $(30 \mathrm{~mL} \times 3)$. The combined organic phasewas washed with $\mathrm{H}_{2} \mathrm{O}(50 \mathrm{~mL} \times 2)$, brine $(50 \mathrm{~mL})$, dried over anhydrous $\mathrm{Na}_{2} \mathrm{SO}_{4}$, and concentrated in vacuo. The residue was purified by silica gel chromatography using hexane/EtOAc as the eluent.

(a): Procedure A
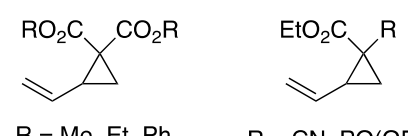
$\mathrm{R}=\mathrm{CN}, \mathrm{PO}(\mathrm{OEt})_{2}$

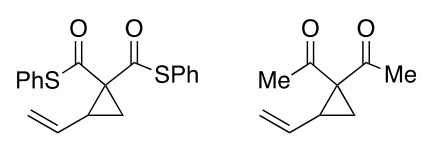

(b): Known in the Literature
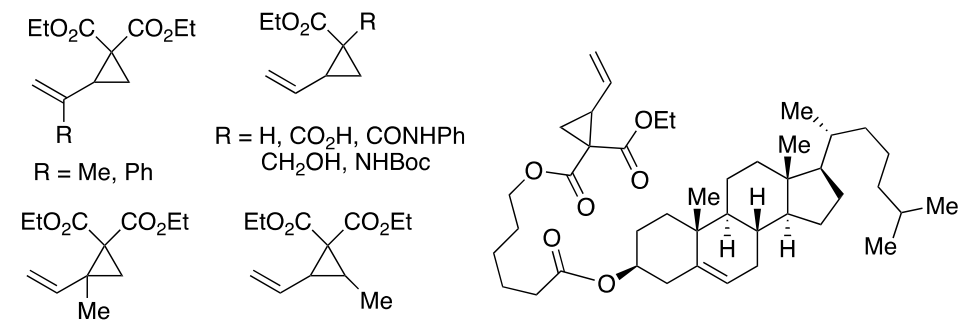

Figure S1. Known vinylcyclopropanes synthesized according to Procedure A or methods in literature. ${ }^{3}$

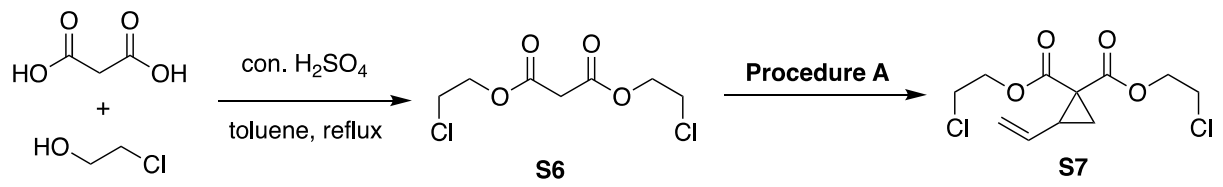

A solution of malonic acid (4.0 g, $38.5 \mathrm{mmol})$, 2-chloroethanol (13.0 mL, $206 \mathrm{mmol})$ and concentrated $\mathrm{H}_{2} \mathrm{SO}_{4}(0.5 \mathrm{~mL})$ in anhydrous toluene $(20.0 \mathrm{~mL})$ was heated to reflux for $3 \mathrm{~h}$. After cooling to room temperature, the reaction was carefully quenched by slow addition of $10 \% \mathrm{NaHCO}_{3}$. The layers were separated, and the aqueous phase was extracted with ether $(50 \mathrm{~mL} \times 3)$. The combined organic phase was washed with $5 \% \mathrm{NaHCO}_{3}(50$ $\mathrm{mL} \times 2), \mathrm{H}_{2} \mathrm{O}(50 \mathrm{~mL} \times 2)$ and brine $(50 \mathrm{~mL})$, dried over anhydrous $\mathrm{Na}_{2} \mathrm{SO}_{4}$, concentrated in vacuo to give crude $\mathbf{S 6}$ as a colorless oil $(5.2 \mathrm{~g})$, which was used without further purification. S7 was then synthesized according to Procedure A. Colorless oil, $4.34 \mathrm{~g}, 40 \%$ yield for two steps. ${ }^{1} \mathbf{H}$ NMR $\left(400 \mathrm{MHz}, \mathrm{CDCl}_{3}\right) \delta 5.48$ (ddd, $\left.J=17.0,10.2,8.1 \mathrm{~Hz}, 1 \mathrm{H}\right)$, $5.32(\mathrm{dd}, J=17.0,1.5 \mathrm{~Hz}, 1 \mathrm{H}), 5.17(\mathrm{dd}, J=10.2,1.5 \mathrm{~Hz}, 1 \mathrm{H}), 4.50-4.28(\mathrm{~m}, 4 \mathrm{H}), 3.90$ $-3.40(\mathrm{~m}, 4 \mathrm{H}), 2.65(\mathrm{dd}, J=9.0,7.7 \mathrm{~Hz}, 1 \mathrm{H}), 1.79(\mathrm{dd}, J=7.7,5.0 \mathrm{~Hz}, 1 \mathrm{H}), 1.64$ (dd, $J$ 
$=9.0,5.0 \mathrm{~Hz}, 1 \mathrm{H}) .{ }^{13} \mathbf{C} \mathbf{N M R}\left(101 \mathrm{MHz}, \mathrm{CDCl}_{3}\right) \delta 169.0,166.7,132.4,119.2,65.1,65.0$, $41.3,41.2,35.6,31.8,20.8$. DART-TOF-LC/MS m/z calcd. for $\mathrm{C}_{11} \mathrm{H}_{18} \mathrm{Cl}_{2} \mathrm{NO}_{4}\left[\mathrm{M}+\mathrm{NH}_{4}{ }^{+}\right]$ 298.0613, found 298.0611.

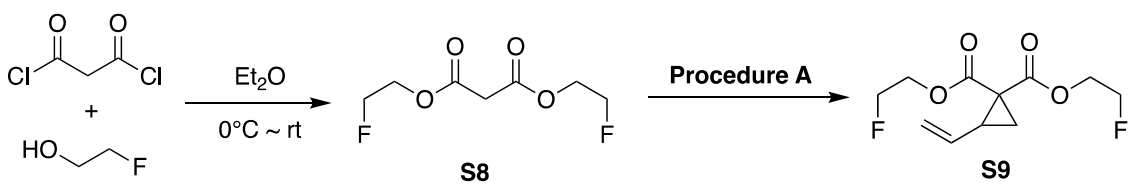

To a solution of 2-fluoroethanol $(1.41 \mathrm{~mL}, 24.0 \mathrm{mmol})$ in anhydrous $\mathrm{Et}_{2} \mathrm{O}(100 \mathrm{~mL})$ at $0{ }^{\circ} \mathrm{C}$, malonyl chloride $(2.82 \mathrm{~g}, 38.5 \mathrm{mmol})$ was added dropwise. The reaction was vigorously stirred at room temperature. After $12 \mathrm{~h}$, the reaction was carefully quenched by slow addition of $10 \% \mathrm{NaHCO}_{3}$. The layers were separated, and the aqueous phase was extracted with ether $(50 \mathrm{~mL} \times 2)$. The combined organic layer was washed with $5 \%$ $\mathrm{NaHCO}_{3}(100 \mathrm{~mL}), \mathrm{H}_{2} \mathrm{O}(100 \mathrm{~mL})$ and brine $(100 \mathrm{~mL})$, dried over anhydrous $\mathrm{Na}_{2} \mathrm{SO}_{4}$, concentrated in vacuo to give crude $\mathbf{S 8}$ as a colorless oil, which was used without further purification. S9 was then synthesized according to Procedure A. Colorless oil, $2.89 \mathrm{~g}, 57 \%$ yield for two steps. ${ }^{1} \mathbf{H}$ NMR $\left(400 \mathrm{MHz}, \mathrm{CDCl}_{3}\right) \delta 5.47$ (ddd, $\left.J=17.0,10.1,8.1 \mathrm{~Hz}, 1 \mathrm{H}\right)$, $5.36-5.28(\mathrm{~m}, 1 \mathrm{H}), 5.19-5.15(\mathrm{~m}, 1 \mathrm{H}), 4.68-4.62(\mathrm{~m}, 2 \mathrm{H}), 4.56-4.52(\mathrm{~m}, 2 \mathrm{H}), 4.51$ $-4.26(\mathrm{~m}, 4 \mathrm{H}), 2.65(\mathrm{dd}, J=9.0,7.9 \mathrm{~Hz}, 1 \mathrm{H}), 1.79(\mathrm{dd}, J=7.7,5.0 \mathrm{~Hz}, 1 \mathrm{H}), 1.64$ (dd, $J$ $=9.0,5.0 \mathrm{~Hz}, 1 \mathrm{H}) .{ }^{13} \mathbf{C ~ N M R}\left(101 \mathrm{MHz}, \mathrm{CDCl}_{3}\right) \delta 169.2,166.9,132.4,119.2,81.9(\mathrm{~d}, J=$ $12.2 \mathrm{~Hz}), 80.2(\mathrm{~d}, J=12.2 \mathrm{~Hz}), 64.5(\mathrm{~d}, J=14.7 \mathrm{~Hz}), 64.2(\mathrm{~d}, J=14.7 \mathrm{~Hz}), 35.6,31.8$, 20.8. ${ }^{19} \mathbf{F}$ NMR $\left(376 \mathrm{MHz}, \mathrm{CDCl}_{3}\right) \delta-224.7,-224.9$. DART-TOF-LC/MS m/z calcd. for $\mathrm{C}_{11} \mathrm{H}_{18} \mathrm{~F}_{2} \mathrm{NO}_{4}\left[\mathrm{M}+\mathrm{NH}_{4}{ }^{+}\right]$266.1204, found 266.1207.

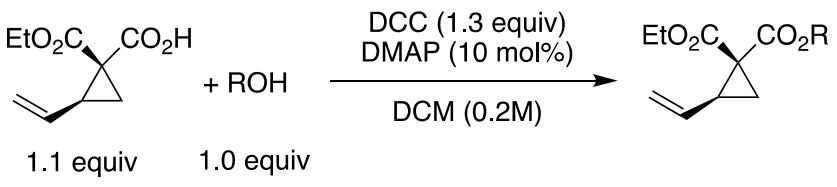

Procedure B: To a solution of 1-(ethoxycarbonyl)-2-vinylcyclopropane-1-carboxylic $\operatorname{acid}^{3 \mathrm{~b}}$ (1.1 equiv.) in anhydrous DCM $(0.2 \mathrm{M})$ at $0{ }^{\circ} \mathrm{C}$, appropriate alcohol (1.0 equiv), DMAP ( 0.10 equiv.), and DCC (1.3 equiv.) was sequentially added. The mixture was then vigorously stirred at room temperature for $12 \mathrm{~h}$. The white precipitate was filtered off and the filtrate was concentrated in vacuo. The residue was purified by silica gel chromatography using hexane/EtOAc as the eluent.

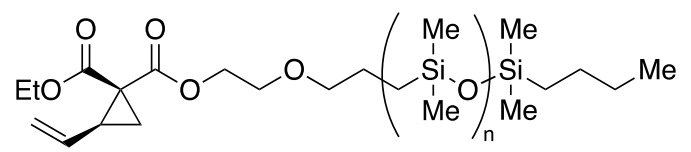

Synthesized from 1-(ethoxycarbonyl)-2-vinylcyclopropane-1-carboxylic acid and 1 $\mathrm{kDa}$ monocarbinol terminated polydimethylsiloxane $(5.0 \mathrm{mmol}$ scale). Colorless oil, $5.23 \mathrm{~g}, 88 \%$ yield. ${ }^{1} \mathbf{H}$ NMR $\left(400 \mathrm{MHz}, \mathrm{CDCl}_{3}\right) \delta 5.44(\mathrm{ddd}, J=17.0,10.2,8.3 \mathrm{~Hz}, 1 \mathrm{H})$, $5.29(\mathrm{dd}, J=17.2,1.6 \mathrm{~Hz}, 1 \mathrm{H}), 5.13(\mathrm{dd}, J=10.1,1.7 \mathrm{~Hz}, 1 \mathrm{H}), 4.35-4.07(\mathrm{~m}, 4 \mathrm{H}), 3.62$ (t, $J=5.0 \mathrm{~Hz}, 2 \mathrm{H}), 3.41(\mathrm{t}, J=7.0 \mathrm{~Hz}, 2 \mathrm{H}), 2.60(\mathrm{q}, J=8.3 \mathrm{~Hz}, 1 \mathrm{H}), 1.71(\mathrm{dd}, J=7.6$, $4.9 \mathrm{~Hz}, 1 \mathrm{H}), 1.64-1.50(\mathrm{~m}, 3 \mathrm{H}), 1.36-1.16(\mathrm{~m}, 7 \mathrm{H}), 0.92-0.78(\mathrm{~m}, 3 \mathrm{H}), 0.59-0.46$ (m, 4H), $0.09-0.03(\mathrm{~m}, 89 \mathrm{H}) .{ }^{13} \mathbf{C}$ NMR $\left(101 \mathrm{MHz}, \mathrm{CDCl}_{3}\right) \delta$ 168.6, 166.2, 132.0, 117.5, 
73.1, 67.2, 63.7, 60.4, 34.8, 30.3, 25.3, 24.4, 22.4, 19.5, 16.9, 13.1, 13.0, 12.8, 0.4, 0.1, 0.0, $-0.4,-0.9,-1.0 . M_{\mathrm{n}}(\mathrm{NMR})=1.4 \mathrm{kDa}$.

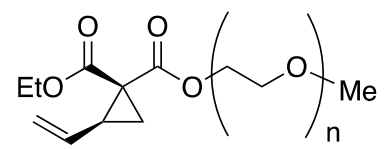

Synthesized from 1-(ethoxycarbonyl)-2-vinylcyclopropane-1-carboxylic acid and PEG 550 (5.0 mmol scale). Colorless oil, 5.23g, 88\% yield. ${ }^{1} \mathbf{H}$ NMR $\left(400 \mathrm{MHz}, \mathrm{CDCl}_{3}\right) \delta$ ${ }^{1} \mathrm{H}$ NMR (400 MHz, Chloroform- $d$ ) $\delta 5.43$ (ddd, $\left.J=17.0,10.1,8.2 \mathrm{~Hz}, 1 \mathrm{H}\right), 5.28$ (dd, $J=$ $17.1,1.6 \mathrm{~Hz}, 1 \mathrm{H}), 5.13(\mathrm{dd}, J=10.0,1.7 \mathrm{~Hz}, 1 \mathrm{H}), 4.33-4.13(\mathrm{~m}, 4 \mathrm{H}), 3.68(\mathrm{t}, J=4.9 \mathrm{~Hz}$, $2 \mathrm{H}), 3.65-3.61(\mathrm{~m}, 34 \mathrm{H}), 3.55-3.52(\mathrm{~m}, 2 \mathrm{H}), 3.37(\mathrm{~s}, 3 \mathrm{H}), 2.58(\mathrm{q}, J=8.3 \mathrm{~Hz}, 1 \mathrm{H})$, $1.70(\mathrm{dd}, J=7.6,4.9 \mathrm{~Hz}, 1 \mathrm{H}), 1.56(\mathrm{dd}, J=9.0,4.9 \mathrm{~Hz}, 1 \mathrm{H}), 1.25(\mathrm{t}, J=7.1 \mathrm{~Hz}, 3 \mathrm{H}) .{ }^{13} \mathrm{C}$ NMR $\left(101 \mathrm{MHz}, \mathrm{CDCl}_{3}\right) \delta 1169.6,167.2,133.0,118.6,71.9,70.63,70.60,70.57,70.51$, $68.8,64.6,61.5,59.0,35.8,31.3,20.5,14.2 . M_{\mathrm{n}}(\mathrm{NMR})=625$.

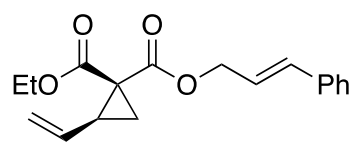

Synthesized from 1-(ethoxycarbonyl)-2-vinylcyclopropane-1-carboxylic acid and cinnamyl alcohol (2.0 mmol scale). Colorless oil, $0.49 \mathrm{~g}, 82 \%$ yield. ${ }^{1} \mathbf{H}$ NMR $(400 \mathrm{MHz}$, $\left.\mathrm{CDCl}_{3}\right) \delta 7.35-7.14(\mathrm{~m}, 5 \mathrm{H}), 6.59(\mathrm{~d}, J=15.8 \mathrm{~Hz}, 1 \mathrm{H}), 6.24-6.13(\mathrm{~m}, 1 \mathrm{H}), 5.36(\mathrm{dd}, J$ $=10.0,8.1 \mathrm{~Hz}, 1 \mathrm{H}), 5.23(\mathrm{dd}, J=17.0,1.6 \mathrm{~Hz}, 1 \mathrm{H}), 5.07(\mathrm{dd}, J=10.0,1.6 \mathrm{~Hz}, 1 \mathrm{H}), 4.80$ $-4.65(\mathrm{~m}, 2 \mathrm{H}), 4.24-4.05(\mathrm{~m}, 2 \mathrm{H}), 2.54(\mathrm{dd}, J=9.0,7.6 \mathrm{~Hz}, 1 \mathrm{H}), 1.66(\mathrm{dd}, J=7.6,4.9$ $\mathrm{Hz}, 1 \mathrm{H}), 1.52(\mathrm{dd}, J=9.0,4.9 \mathrm{~Hz}, 1 \mathrm{H}), 1.18(\mathrm{t}, J=7.1 \mathrm{~Hz}, 3 \mathrm{H}) .{ }^{13} \mathbf{C}$ NMR $(100 \mathrm{MHz}$, $\left.\mathrm{CDCl}_{3}\right) \delta 169.5,167.3,136.1,134.3,133.0,128.6,128.1,126.6,122.7,118.6,66.1,61.5$, 35.9, 31.3, 20.5, 14.2. DART-TOF-LC/MS m/z calcd. for $\mathrm{C}_{18} \mathrm{H}_{24} \mathrm{NO}_{4}\left[\mathrm{M}+\mathrm{NH}_{4}{ }^{+}\right]$ 318.1705 , found 318.1705 .

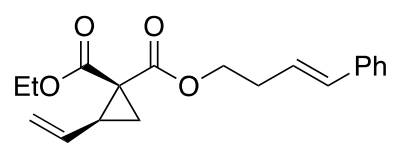

Synthesized from 1-(ethoxycarbonyl)-2-vinylcyclopropane-1-carboxylic acid and trans-4-phenylbut-3-en-1-ol (2.0 mmol scale). Colorless oil, $0.44 \mathrm{~g}, 70 \%$ yield. ${ }^{\mathbf{1}} \mathbf{H}$ NMR $\left(400 \mathrm{MHz}, \mathrm{CDCl}_{3}\right) \delta 7.30-7.09(\mathrm{~m}, 5 \mathrm{H}), 6.39(\mathrm{~d}, J=15.9 \mathrm{~Hz}, 1 \mathrm{H}), 6.07(\mathrm{dt}, J=15.7$, $7.0 \mathrm{~Hz}, 1 \mathrm{H}), 5.35$ (ddd, $J=16.6,10.1,8.3 \mathrm{~Hz}, 1 \mathrm{H}), 5.19(\mathrm{dd}, J=17.0,1.6 \mathrm{~Hz}, 1 \mathrm{H}), 5.04$ $(\mathrm{dd}, J=10.0,1.6 \mathrm{~Hz}, 1 \mathrm{H}), 4.31-4.02(\mathrm{~m}, 4 \mathrm{H}), 2.56-2.40(\mathrm{~m}, 3 \mathrm{H}), 1.61(\mathrm{dd}, \mathrm{J}=7.5,4.9$ $\mathrm{Hz}, 1 \mathrm{H}), 1.47(\mathrm{dd}, \mathrm{J}=9.0,4.9 \mathrm{~Hz}, 1 \mathrm{H}), 1.14(\mathrm{t}, \mathrm{J}=7.1 \mathrm{~Hz}, 3 \mathrm{H}) .{ }^{13} \mathbf{C}$ NMR $(100 \mathrm{MHz}$, $\left.\mathrm{CDCl}_{3}\right) \delta 169.6,167.3,137.2,133.1,132.6,128.5,127.3,126.1,125.3,118.5,64.7,61.5$, $35.9,32.3,31.2,20.5,14.2$. DART-TOF-LC/MS m/z calcd. for $\mathrm{C}_{19} \mathrm{H}_{26} \mathrm{NO}_{4}\left[\mathrm{M}+\mathrm{NH}_{4}{ }^{+}\right]$ 332.1862 , found 332.1860 . 


\section{Complementary Optimization of Conditions}

Table S1. Single-electron oxidation of bromide salts to generate bromine radicals for the [3+2] cycloaddition of $\mathbf{1}$ and styrene. ${ }^{a}$

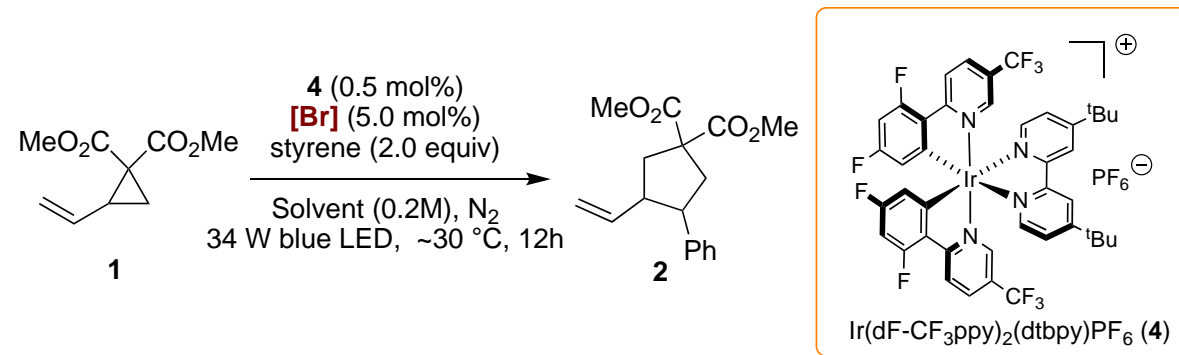

\begin{tabular}{cccc}
\hline Entry & {$[\mathrm{Br}]$} & Solvent & NMR Yield $(\%)^{b}$ \\
1 & $\mathrm{NaBr}$ & MeCN & 0 \\
2 & $\mathrm{KBr}$ & $\mathrm{MeCN}$ & 0 \\
3 & $\mathrm{LiBr}$ & $\mathrm{MeCN}$ & 0 \\
4 & $\mathrm{TBAB}$ & $\mathrm{MeCN}$ & 0 \\
5 & $\mathrm{NaBr}$ & $\mathrm{DMSO}$ & 0 \\
6 & $\mathrm{KBr}$ & $\mathrm{DMSO}$ & 0 \\
7 & $\mathrm{LiBr}$ & $\mathrm{DMSO}$ & 0 \\
8 & $\mathrm{TBAB}$ & $\mathrm{DMSO}$ & 0 \\
9 & $\mathrm{NaBr}$ & $\mathrm{DMF}$ & 0 \\
10 & $\mathrm{KBr}$ & $\mathrm{DMF}$ & 0 \\
11 & $\mathrm{LiBr}$ & $\mathrm{DMF}$ & 0 \\
12 & $\mathrm{TBAB}$ & $\mathrm{DMF}$ & 0 \\
13 & $\mathrm{LiBr}$ & $\mathrm{acetone}$ & 0 \\
14 & $\mathrm{TBAB}$ & acetone & 0 \\
15 & $\mathrm{LiBr}$ & $\mathrm{THF}$ & 0 \\
16 & $\mathrm{TBAB}$ & $\mathrm{THF}$ & 0 \\
17 & $\mathrm{LiBr}$ & $\mathrm{MeOH}$ & 0 \\
18 & $\mathrm{TBAB}$ & $\mathrm{MeOH}$ & 0 \\
\hline
\end{tabular}

${ }^{a}$ General reaction conditions: $1(0.2 \mathrm{mmol})$, styrene $(0.4 \mathrm{mmol}), 4(0.5 \%)$, bromide salt $(5.0 \%)$, anhydrous solvent $(1.0 \mathrm{~mL}), 34 \mathrm{~W}$ Kessil blue LED. ${ }^{b}$ Trimethoxybenzene was used as an internal standard. TBAB $=$ tetrabutylammonium bromide 
Table S2. Solvent effect in the [3+2] cycloaddition of $\mathbf{1}$ and styrene using cinnamyl bromide as the precatalyst. ${ }^{a}$

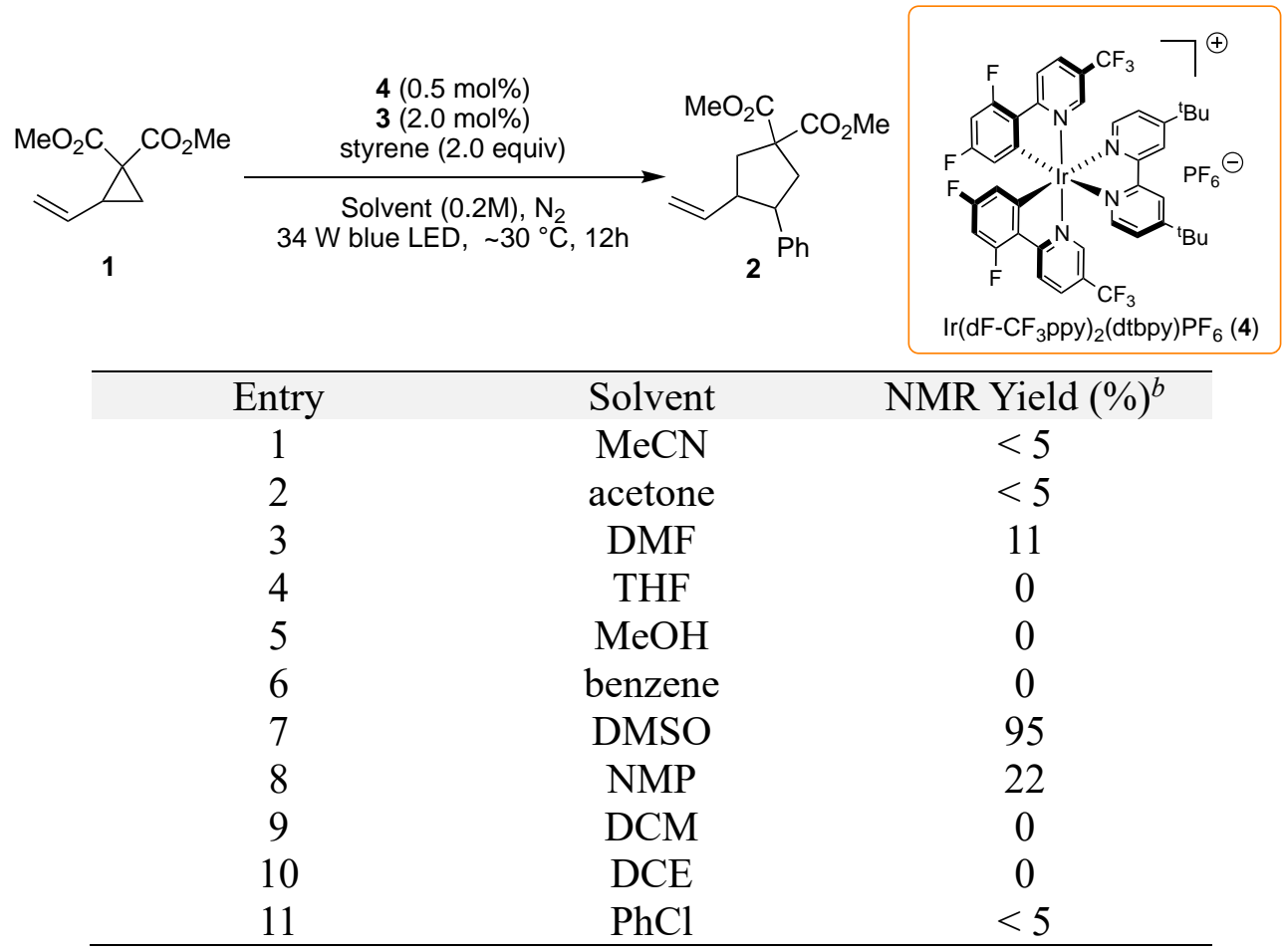

${ }^{a}$ Reaction conditions: $1(0.2 \mathrm{mmol})$, styrene $(0.4 \mathrm{mmol}), 4(0.5 \%)$, cinnamyl bromide 3 $(2.0 \%)$, anhydrous solvent $(1.0 \mathrm{~mL}), 34 \mathrm{~W}$ Kessil blue LED. ${ }^{b}$ Trimethoxylbenzene was used as an internal standard. 
Table S3. Examination of precatalyst (3) loading in the [3+2] cycloaddition of $\mathbf{1}$ and styrene. $^{a}$

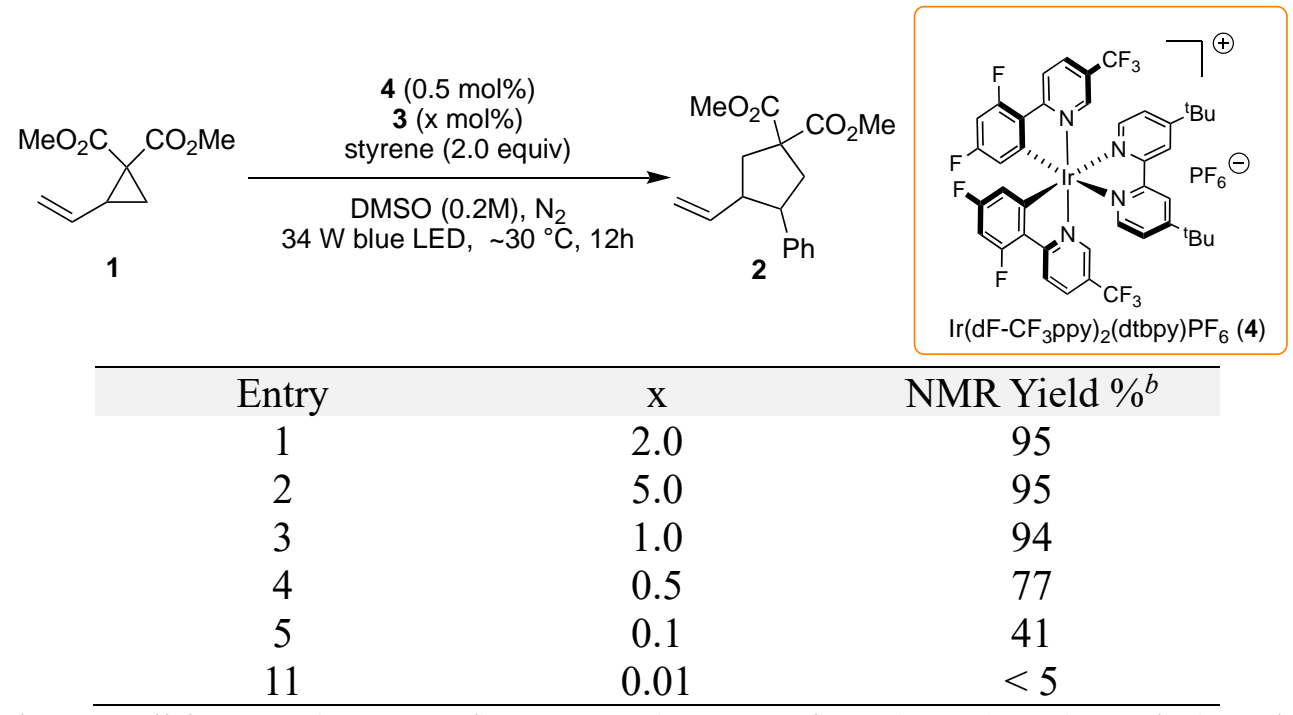

${ }^{a}$ Reaction conditions: 1 (0.2 mmol), styrene $(0.4 \mathrm{mmol}), 4(0.5 \%), 3(\mathrm{x} \mathrm{mol} \%)$, anhydrous DMSO $(1.0 \mathrm{~mL}), 34 \mathrm{~W}$ Kessil blue LED. ${ }^{b}$ Trimethoxylbenzene was used as an internal standard. 


\section{General Reaction Setup and Experimental Procedures}

Procedure $C$ : In a $\mathrm{N}_{2}$-filled glovebox, an oven-dried vial ( $\left.0.5 \mathrm{dram}\right)$ equipped with a magnet stir bar was charged with vinyl- or ethynylcycloproane ( $0.2 \mathrm{mmol}, 1.0$ equiv.), appropriate alkene (2.0 equiv.), $50 \mathrm{uL}$ stock solution of cinnamyl bromide 3 in DMSO (40 $\mathrm{mM}$ for $1.0 \mathrm{~mol} \%$; $80 \mathrm{mM}$ for $2.0 \mathrm{~mol} \%)$, anhydrous DMSO (1.90 mL for $0.1 \mathrm{M} ; 0.90 \mathrm{~mL}$ for $0.2 \mathrm{M} ; 0.40 \mathrm{~mL}$ for $0.4 \mathrm{M})$, and $50 \mathrm{uL}$ stock solution of 4 CzIPN 7 in DMSO $(0.2 \mathrm{mM}$ for $50 \mathrm{ppm} ; 2.0 \mathrm{mM}$ for $500 \mathrm{ppm}$ ). The vial was then tightly capped and removed out of glovebox. The reaction was placed into a photoreactor (HeptatoChem HCK 1006-01-004) equipped with a blue LED (Kessil, $34 \mathrm{~W}$ ). The reactor was cooling by air flow that the temperature was $\left.\sim 30^{\circ} \mathrm{C}\right)$. After $12 \mathrm{~h}$, the reaction mixture was diluted with water $(10 \mathrm{~mL})$ and extracted with ether $(20 \mathrm{~mL} \times 3)$. The combined organic phase was washed with $\mathrm{H}_{2} \mathrm{O}$ $(20 \mathrm{~mL} \times 3)$, brine $(30 \mathrm{~mL})$, dried over anhydrous $\mathrm{Na}_{2} \mathrm{SO}_{4}$, concentrated in vacuo. The residue was then subjected to silica gel chromatography for purification.
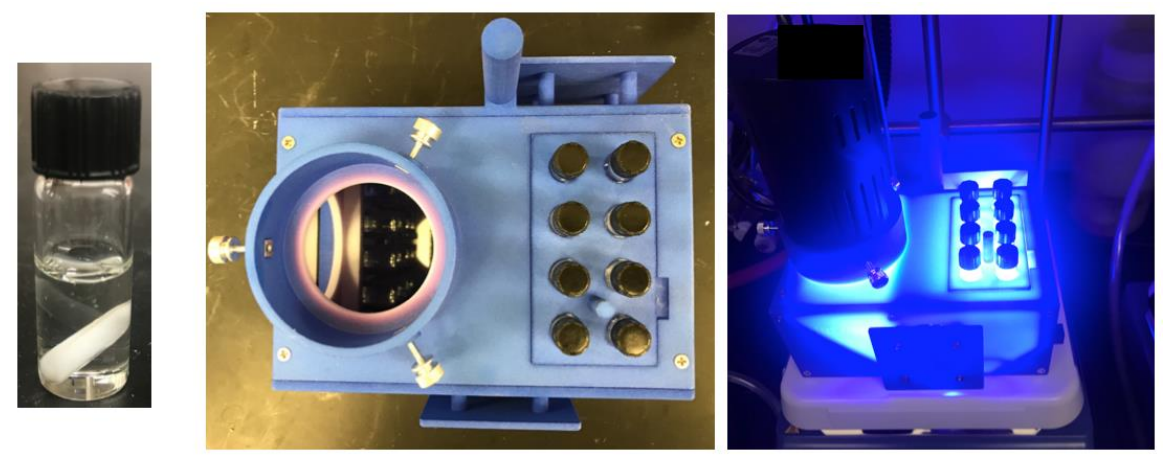

Figure S2. Reaction setup (HeptatoChem HCK 1006-01-004 with Kessil 34 W H-150 blue LED). 


\section{Mechanistic Study}

\subsection{TEMPO-trapping experiment}

In a $\mathrm{N}_{2}$-filled glovebox, an oven-dried vial ( 0.5 dram) equipped with a magnet stir bar was charged with cinnamyl bromide 3 (39.4 mg, $0.2 \mathrm{mmol}$ ), TEMPO (93.8 mg, $0.6 \mathrm{mmol}$ ), anhydrous DMSO (1.0 mL) and 4CzIPN $7(1.6 \mathrm{mg}, 0.002 \mathrm{mmol})$. The vial was then tightly capped and removed out of glovebox. The reaction was placed into a photoreactor (HeptatoChem HCK 1006-01-004) equipped with a blue LED (Kessil, 34 W). The reactor was cooling by air flow that the temperature was $\sim 30{ }^{\circ} \mathrm{C}$ ). After $2 \mathrm{~h}$, an aliquot of $10 \mathrm{uL}$ was taken for ESI-TOF LC/MS analysis. The reaction mixture was diluted with water (10 $\mathrm{mL})$ and extracted with ether $(20 \mathrm{~mL} \times 3)$. The combined organic phase was washed with $\mathrm{H}_{2} \mathrm{O}(20.0 \mathrm{~mL} \times 2)$, brine $(30 \mathrm{~mL})$, dried over anhydrous $\mathrm{Na}_{2} \mathrm{SO}_{4}$, concentrated in vacuo. $32.0 \mathrm{mg}$ (81\% yield) cinnamyl bromide was recovered through silica gel chromatography.

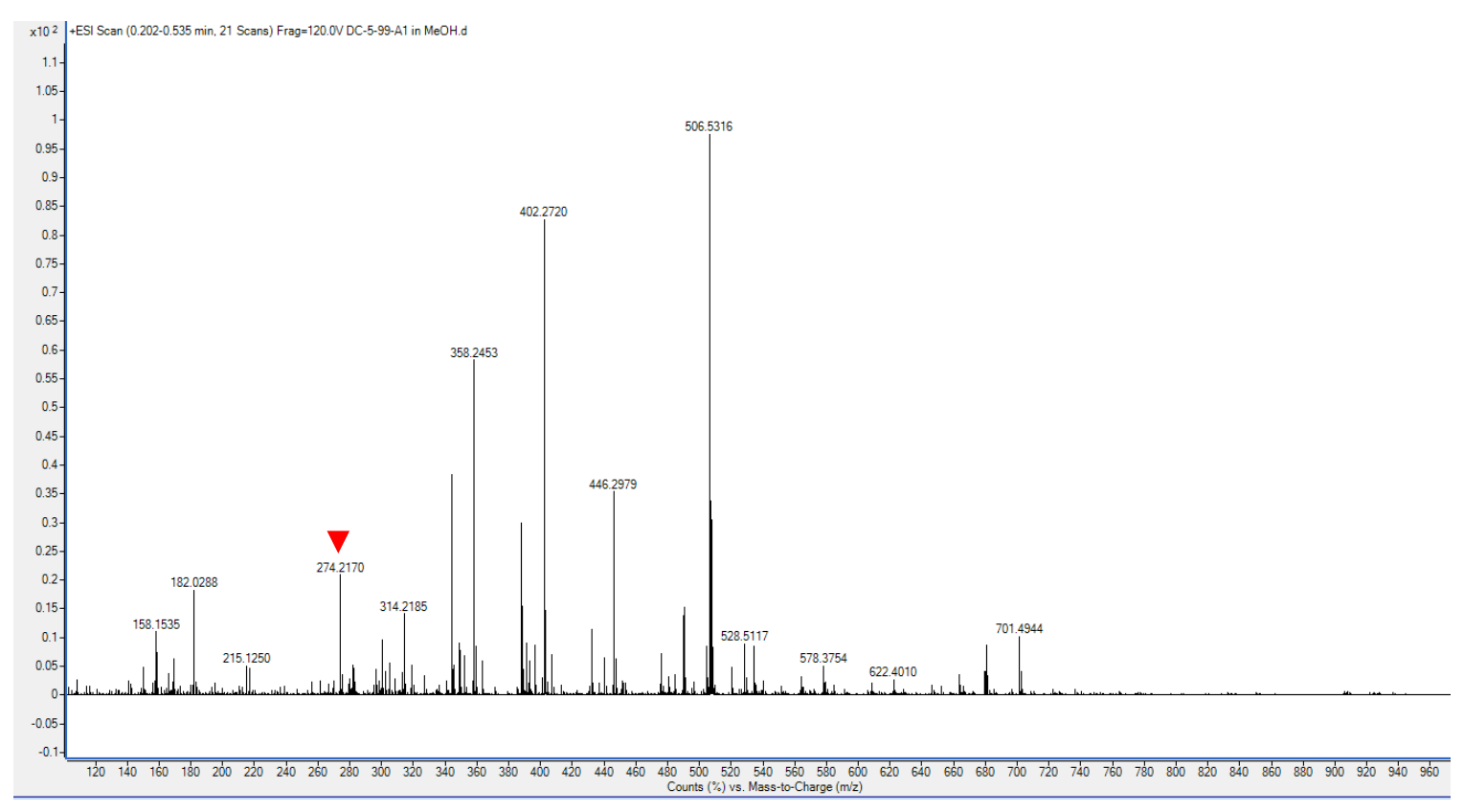

Figure S3. ESI-TOF LC/MS spectrum of aliquot taken from TEMPO-trapping experiment 


\subsection{Investigation of alternative precatalysts}

$\mathrm{n}$ a $\mathrm{N}_{2}$-filled glovebox, an oven-dried vial ( $\left.0.5 \mathrm{dram}\right)$ equipped with a magnet stir bar was charged with vinylcycloproane $1(0.2 \mathrm{mmol}, 1.0$ equiv.), styrene ( $46.0 \mathrm{uL}, 0.4 \mathrm{mmol})$, $50 \mathrm{uL}$ stock solution of 7 in DMSO $(0.2 \mathrm{mM})$, anhydrous DMSO $(0.90 \mathrm{~mL})$, and $50 \mathrm{uL}$ stock solution of precatalyst in DMSO $(40 \mathrm{mM})$. The vial was then tightly capped and removed out of glovebox. The reaction was placed into a photoreactor (HeptatoChem HCK 1006-01-004) equipped with a blue LED (Kessil, $34 \mathrm{~W}$ ). The reactor was cooling by air flow that the temperature was $\sim 30{ }^{\circ} \mathrm{C}$ ). After $12 \mathrm{~h}$, the vial was opened to the air and 1,3,5-trimethoxylbenzene $(16.8 \mathrm{mg}, 0.1 \mathrm{mmol})$ was added as an internal standard. An aliquot of $0.10 \mathrm{~mL}$ was taken for direct ${ }^{1} \mathrm{H}$ NMR analysis to determine the yield.

Table S4. Results of [3+2] cycloaddition of 1 and styrene using alternative precatalyst. $^{a}$

\begin{tabular}{ccccc}
\hline Entry & precatalyst & 7 & light & NMR Yield $(\%)^{b}$ \\
1 & allyl bromide & $\sqrt{ }$ & $\sqrt{ }$ & 0 \\
2 & cinnamyl chloride & $\sqrt{ }$ & $\sqrt{ }$ & 0 \\
3 & NBS & $\sqrt{ }$ & $\sqrt{ }$ & 41 \\
4 & $\mathrm{NBS}$ & $\sqrt{ }$ & $\times$ & 0 \\
5 & $\mathrm{NBS}$ & $\times$ & $\sqrt{ }$ & 0 \\
6 & $\mathrm{NBS}$ & $\times$ & $\times$ & 0 \\
7 & $\mathrm{Br} 2$ & $\sqrt{ }$ & $\sqrt{ }$ & 26 \\
8 & $\mathrm{Br}_{2}$ & $\sqrt{ }$ & $\times$ & 0 \\
9 & $\mathrm{Br}_{2}$ & $\times$ & $\sqrt{ }$ & 16 \\
10 & $\mathrm{Br} 2$ & $\times$ & $\times$ & 0 \\
\hline
\end{tabular}

${ }^{a}$ Reaction conditions: $1(0.2 \mathrm{mmol})$, styrene $(0.4 \mathrm{mmol}), 7(50 \mathrm{ppm})$, precatalyst $(1.0 \%)$, anhydrous DMSO $(1.0 \mathrm{~mL}), 34 \mathrm{~W}$ Kessil blue LED, $12 \mathrm{~h} .{ }^{b}$ Trimethoxylbenzene was used as an internal standard. 


\subsection{Radical epimerization experiment}

In a $\mathrm{N}_{2}$-filled glovebox, an oven-dried vial ( $\left.0.5 \mathrm{dram}\right)$ equipped with a magnet stir bar was charged with cis-1-(ethoxycarbonyl)-2-vinylcyclopropane-1-carboxylic acid (36.8 mg, $0.2 \mathrm{mmol}), 50 \mathrm{uL}$ stock solution of cinnamyl bromide 3 in DMSO $(40 \mathrm{mM})$, anhydrous DMSO $(0.90 \mathrm{~mL})$, and $50 \mathrm{uL}$ stock solution of 4CzIPN 7 in DMSO $(0.2 \mathrm{mM})$. The vial was then tightly capped and removed out of glovebox. The reaction was placed into a photoreactor (HeptatoChem HCK 1006-01-004) equipped with a blue LED (Kessil, 34W). The reactor was cooling by air flow that the temperature was $\left.\sim 30^{\circ} \mathrm{C}\right)$. After $12 \mathrm{~h}$, the reaction mixture was diluted with water $(10 \mathrm{~mL})$ and extracted with $\mathrm{CH}_{2} \mathrm{Cl}_{2}(20 \mathrm{~mL} \times 3)$. The combined organic phase was washed with brine $(30.0 \mathrm{~mL})$, dried over anhydrous $\mathrm{Na}_{2} \mathrm{SO}_{4}$, and concentrated in vacuo. Crude ${ }^{1} \mathrm{H}$ NMR was taken to determine the cis/trans ratio.
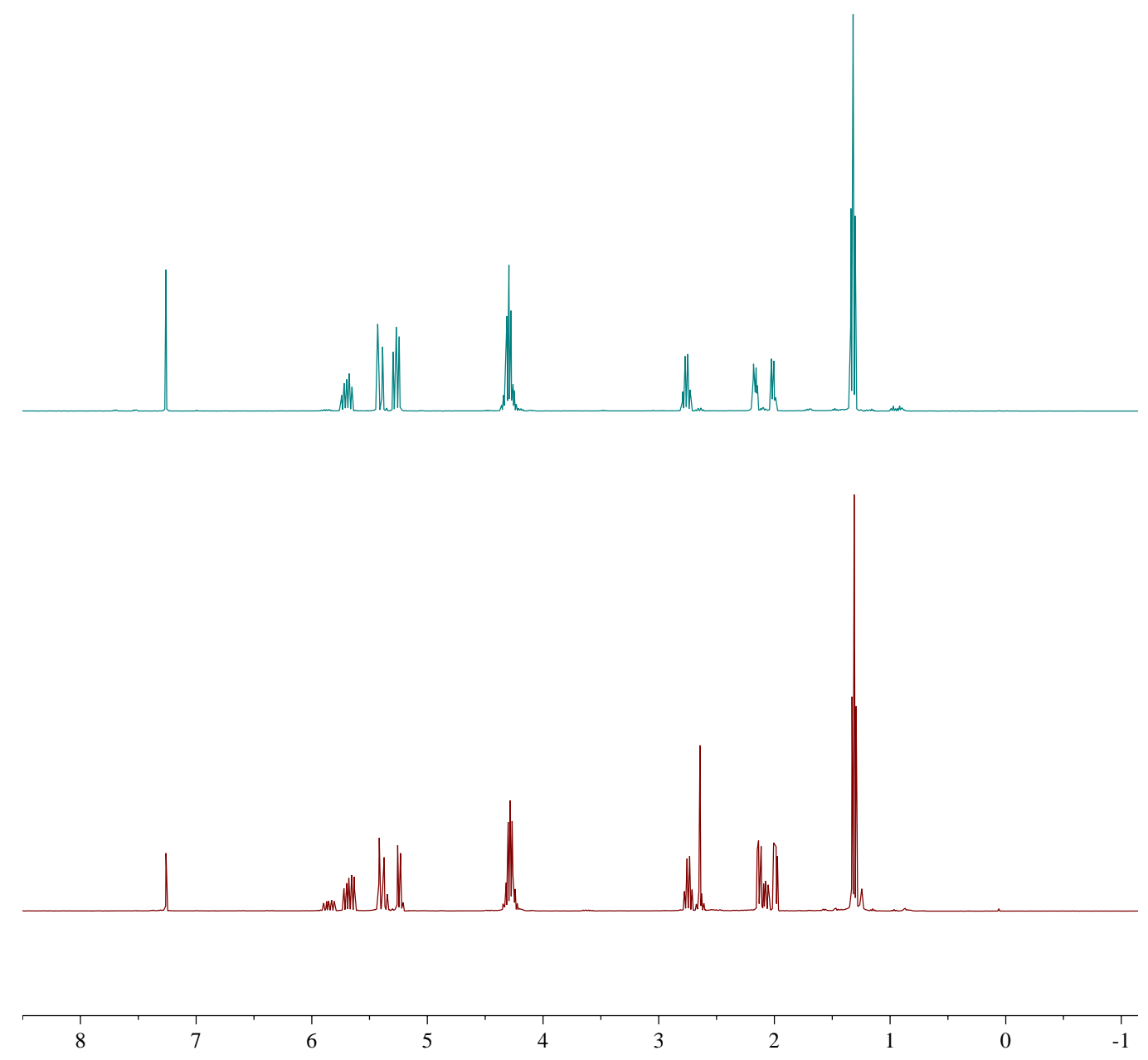

Figure S4. Comparison of ${ }^{1} \mathrm{H}$ NMR spectra before (teal, top) and after (bottom) radical epimerization 


\subsection{Cyclic voltammetry}

Cyclic voltammetry (CV) was performed on an Interface 1010B Potentiostat (Gamry Instruments) with a scan rate of $0.10 \mathrm{~V} / \mathrm{s}$. A solution of $\mathrm{Ag} / \mathrm{AgNO}_{3}(0.01 \mathrm{M})$ and $0.1 \mathrm{M}$ $\mathrm{Bu}_{4} \mathrm{NPF}_{6}$ in $\mathrm{MeCN}$ was used as the reference electrode (Gamry Instruments, part no. 930-00059). A 0.1 M Bu4NPF 6 in $\mathrm{MeCN}_{4}$ was used as the electrolyte for cinnamyl bromide solution $(0.05 \mathrm{M})$. A platinum disk was used for the working electrode (Gamry Instruments, part no. 932-00024) and a platinum wire was used as the counter electrode (Gamry Instruments, part no. 990-00193).

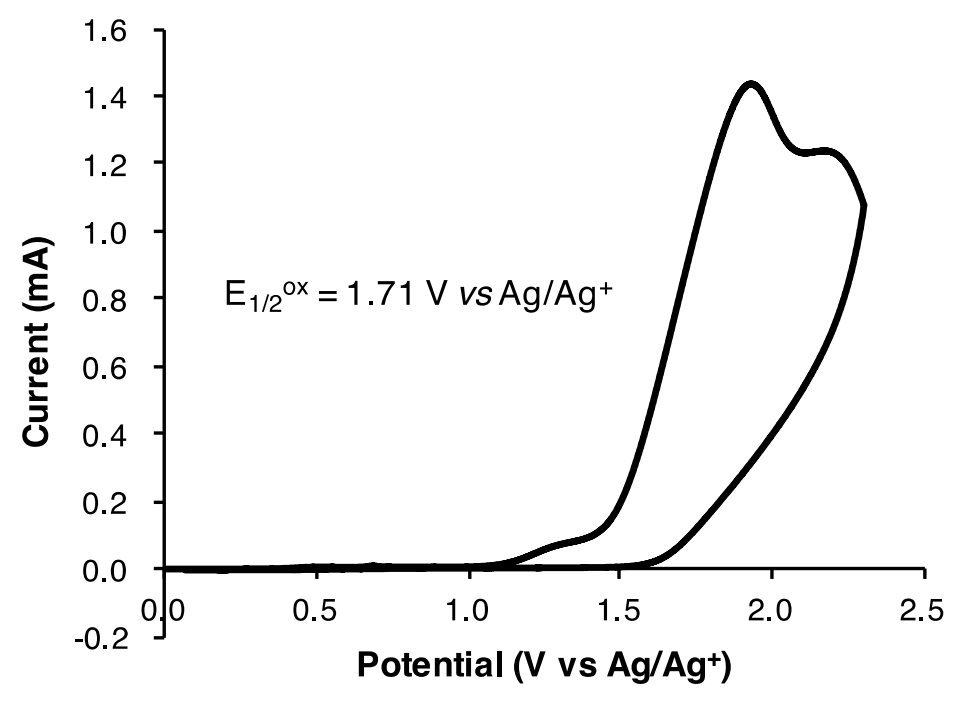

Figure S5. CV spectrum of cinnamyl bromide $\left(\mathrm{E}_{1 / 2}{ }^{\mathrm{ox}}=1.71 \mathrm{vs} \mathrm{Ag} / \mathrm{Ag}^{+}=2.01 \mathrm{~V}\right.$ vs SCE$)$ 


\subsection{Stern-Volmer quenching experiment}

Stern-Volmer experiments were operated on pectrofluorometer FS5 (Edinburgh Instruments) and with different solutions containing $10.0 \mathrm{uM} \mathrm{4CzIPN} 7$ in DMSO and $\mathrm{x}$ $\mathrm{mM} 3((\mathrm{x}=1.5,10,20,40))$, or vinylcyclopropane $\mathbf{1}(\mathrm{x}=10,20,30,50)$, or styrene $(\mathrm{x}=10$, $20,30,50)$ in DMSO under irradiation at $380 \mathrm{~nm}$. The luminescence was measured at 560 $\mathrm{nm}$. Averaged data from three different runs were used for graphical representation.

Table S5. Luminescence quenching of 4CzIPN 7 at variable concentration of $\mathbf{3}$

\begin{tabular}{|c|c|c|c|c|c|c|}
\hline $\mathbf{3 a}(\mathrm{mM})$ & 0 & 1 & 5 & 10 & 20 & 40 \\
\hline $\mathrm{I}_{0} / \mathrm{I}$ & 1.0000 & 1.0344 & 1.1250 & 1.2273 & 1.4040 & 1.6488 \\
\hline
\end{tabular}

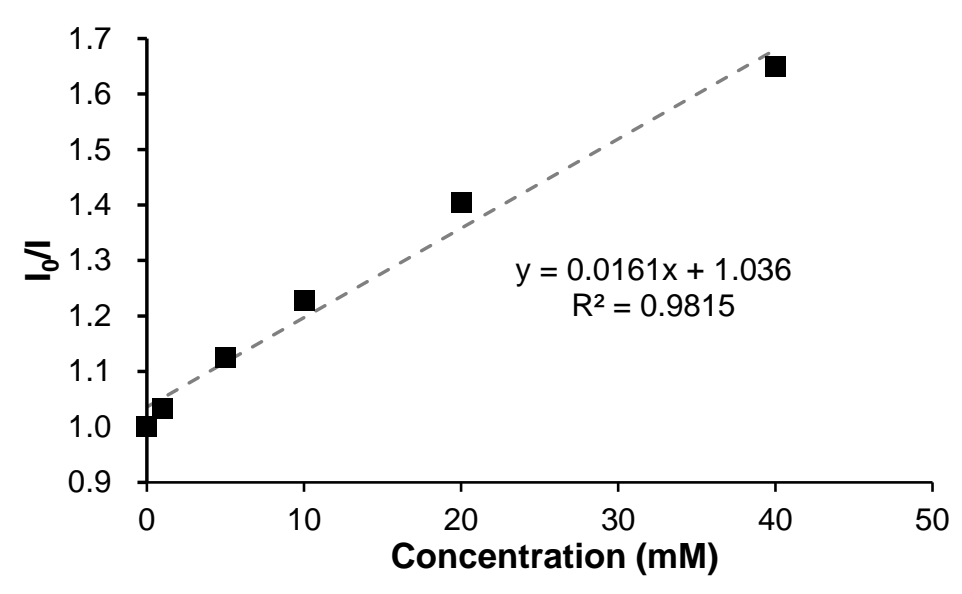

Figure S6. Stern-Volmer plots of 4CzIPN 7 at variable concentration of 3. 
Table S6. Luminescence quenching of $4 \mathrm{CzIPN} 7$ at variable concentration of vinylcyclopropane $\mathbf{1}$

\begin{tabular}{|c|c|c|c|c|c|}
\hline $\mathbf{1}(\mathrm{mM})$ & 0 & 10 & 20 & 30 & 50 \\
\hline $\mathrm{I}_{0} / \mathrm{I}$ & 1.0000 & 1.0076 & 1.0013 & 1.0036 & 0.9949 \\
\hline
\end{tabular}

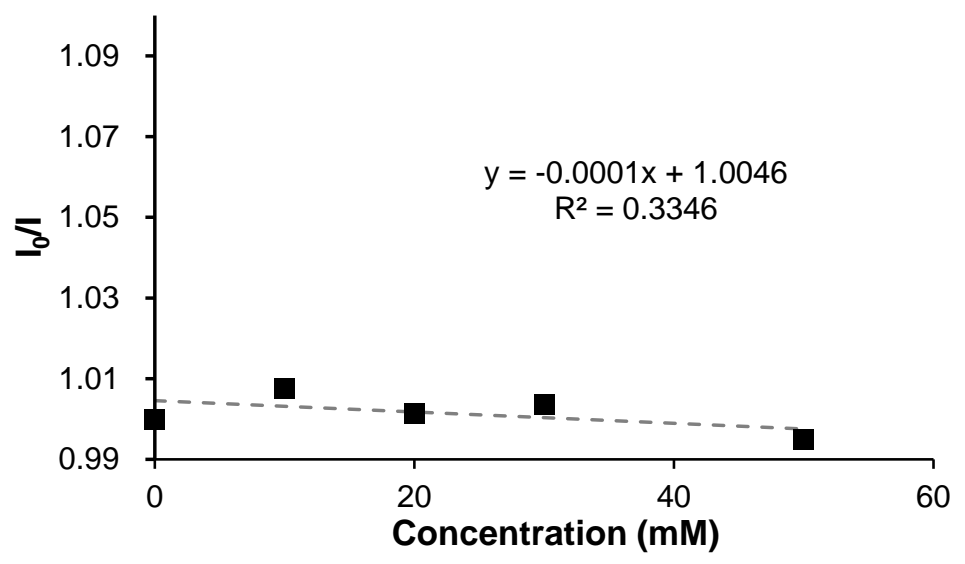

Figure S7. Stern-Volmer plots of 4CzIPN 7 at variable concentration of vinylcyclopropane 1. 
Table S7. Luminescence quenching of 4CzIPN 7 at variable concentration of styrene

\begin{tabular}{|c|c|c|c|c|c|}
\hline styrene $(\mathrm{mM})$ & 0 & 10 & 20 & 30 & 50 \\
\hline $\mathrm{I}_{0} / \mathrm{I}$ & 1.0000 & 1.0076 & 1.0013 & 1.0036 & 0.9949 \\
\hline
\end{tabular}

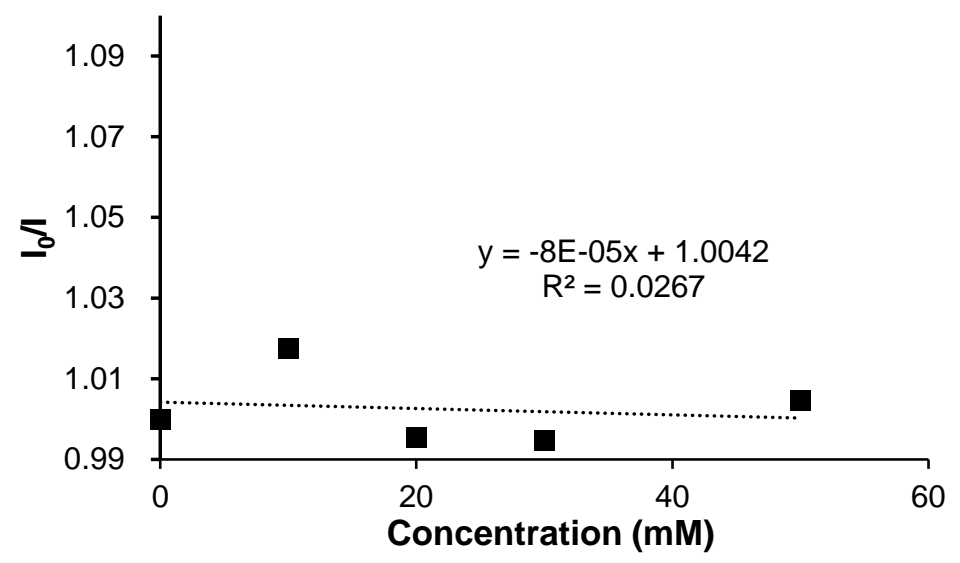

Figure S8. Stern-Volmer plots of $4 \mathrm{CzIPN} 7$ at variable concentration of styrene. 


\section{6 “On/off” experiment}

The reaction was set up in a $\mathrm{N}_{2}$-filled glovebox according to the Procedure $\mathbf{C}$. The reaction mixture was irradiated for an hour. An aliquot of $0.10 \mathrm{~mL}$ was taken from the reaction mixture and injected into a vial containing $0.50 \mathrm{~mL}$ of $\mathrm{CDCl}_{3}$ containing trimethoxylbenzene $(4.2 \mathrm{mg}$ ) as the internal standard. The yield of product was determined by crude ${ }^{1} \mathrm{H}$ NMR analysis. The reaction mixture was then wrapped by aluminum foil and re-subject to $34 \mathrm{~W}$ Kessil Blue LED. The yields later on were determined in the same way after some time light on or off.

Table S8. Results of “on/off” experiment

\begin{tabular}{ccc}
\hline Entry & Time $(\mathrm{h})$ & ${\text { NMR Yield }(\%)^{a}}^{a}$ \\
1 & 0 & 0 \\
2 & 1 & 15 \\
3 & 2 (off) & 15 \\
4 & 3 & 27 \\
5 & 4 (off) & 27 \\
6 & 6 & 46 \\
7 & 7 (off) & 46 \\
8 & 9 & 60 \\
\hline
\end{tabular}

${ }^{a}$ Trimethoxybenzene was used as an internal standard. 


\subsection{Determination of quantum yield $(\Phi)$}

In a $\mathrm{N}_{2}$-filled glovebox, an oven-dried J-Young NMR tube equipped with a micro stir bar was charged with vinylcyclopropane 1 (18.4 $\mathrm{mg}, 0.10 \mathrm{mmol})$, styrene (23.0 uL, 0.20 $\mathrm{mmol}), 25 \mathrm{uL}$ stock solution of cinnamyl bromide 3 in DMSO (40 mM), anhydrous DMSO $(0.45 \mathrm{~mL})$, and $25 \mathrm{uL}$ stock solution of 4CzIPN 7 in DMSO $(0.2 \mathrm{mM})$. The sealed NMR tube was removed out of the glovebox and irradiated at $419 \mathrm{~nm}$. After $8 \mathrm{~h}(\mathrm{t}=28800 \mathrm{~s}), 8.0$ $\mathrm{mg}$ trimethoxylbenzene was added to the NMR tube. An aliquot $(0.050 \mathrm{~mL})$ was then taken and injected into a HPLC vial containing $0.55 \mathrm{~mL}$ of $\mathrm{CDCl}_{3}$ for direct ${ }^{1} \mathrm{H}-\mathrm{NMR}$ analysis. The NMR yield was determined to be $5.2 \%$ (Fig. S8).

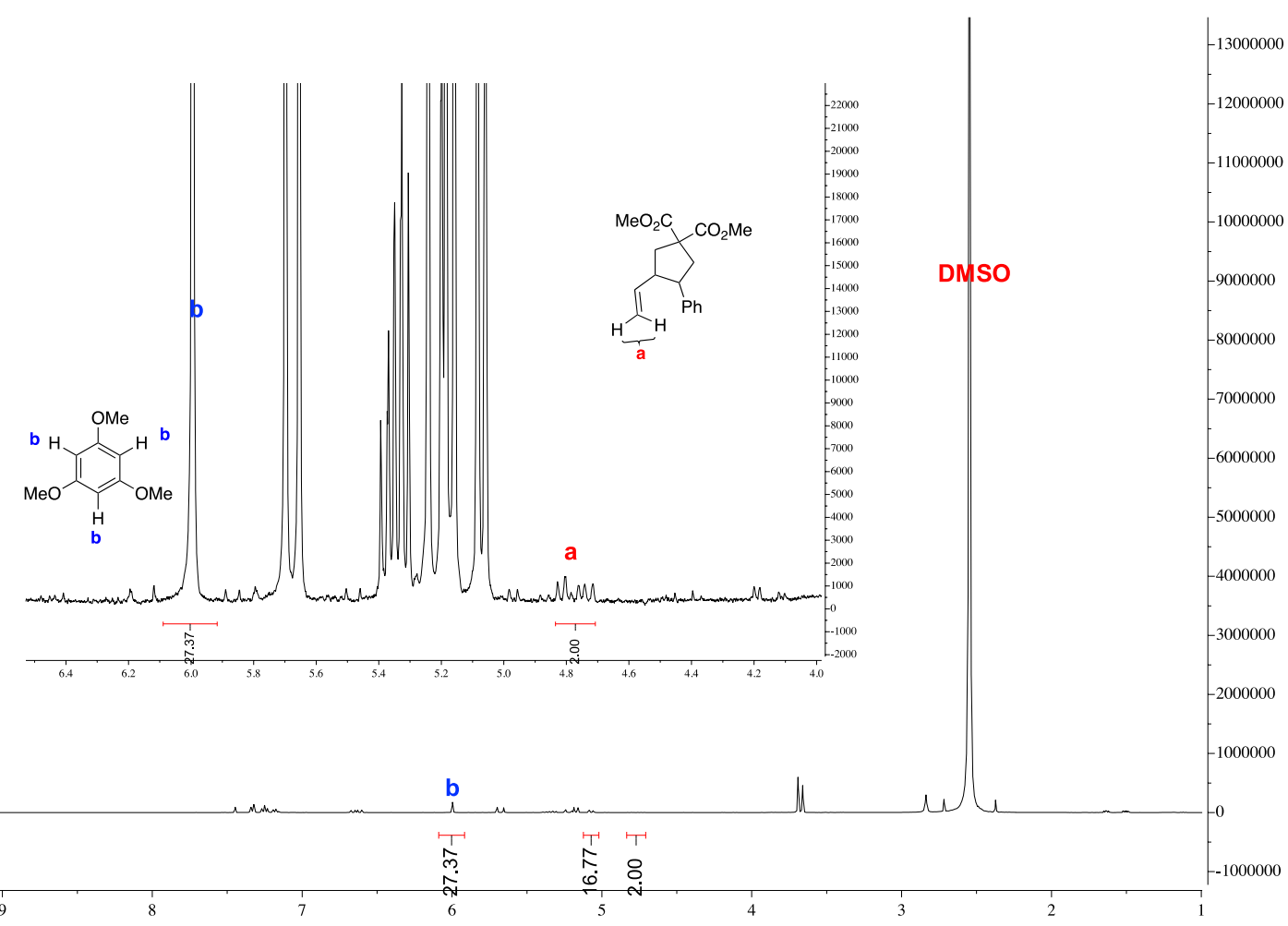

Figure S9. Crude ${ }^{1} \mathrm{H}$ NMR analysis.

The quantum yield $(\Phi)$ can be calculated by using the equation as shown below:

$$
\Phi=\frac{\text { mol product }}{\text { photon flux } \cdot \mathrm{f} \cdot \mathrm{t}}
$$

The photon flux at $419 \mathrm{~nm}$ for the aforementioned setup was previously determined to be $5.2 \times 10^{-9}$ einstein/s.

Fraction of light absorbed (f) was calculated by $\mathrm{f}=1-10^{-\mathrm{A}}$. A is the absorbance of $\mathrm{PC}$ in a specific solvent and concentration. In this experiment, the concentration of 4CzIPN was $10 \mathrm{uM}$. A was determined to be 0.09146 at $419 \mathrm{~nm}$ for a $10 \mathrm{uM}$ solution of 4CzIPN in DMSO (Fig. S10), therefore, $\mathrm{f}=0.1899$.

The quantum yield was calculated by: 


$$
\begin{aligned}
\Phi & =\frac{\text { mol product }}{\text { photon flux } \cdot \mathrm{f} \cdot \mathrm{t}} \\
& =\frac{5.2 \% \times 0.1 / 1000}{5.2 \times 10^{-9} \times 0.1899 \times 28800}=18.3 \%
\end{aligned}
$$

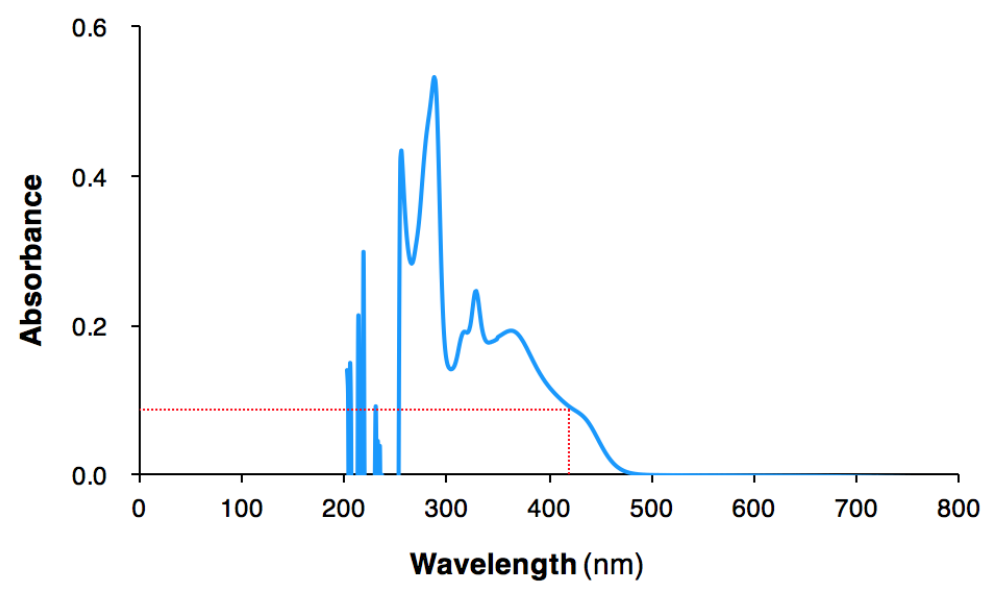

Figure S10. UV-Vis Spectrum of 4CZIPN in DMSO (10 uM) 


\section{Characterization of Products}

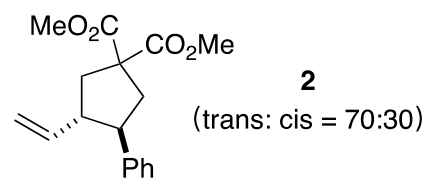

Prepared according to Procedure $\boldsymbol{C}$. Colorless oil (50.4 $\mathrm{mg}, 88 \%$ yield) as a mixture of diastereomers (trans : $\operatorname{cis}=70: 30) . \mathrm{R}_{\mathrm{f}}=0.35$ (10\% EtOAc in hexane).

${ }^{1} \mathbf{H}$ NMR (400 MHz, $\left.\mathrm{CDCl}_{3}\right) \delta 7.27-6.95(\mathrm{~m}, 5 \mathrm{H}), 5.57$ (ddd, $J=16.8,10.8,7.0 \mathrm{~Hz}$, $0.30 \mathrm{H}), 5.29$ (ddd, $J=17.1,10.3,8.0 \mathrm{~Hz}, 0.70 \mathrm{H}), 4.90-4.58(\mathrm{~m}, 2.0 \mathrm{H}), 3.80-3.55(\mathrm{~m}$, $6.0 \mathrm{H}), 3.37(\mathrm{dt}, J=10.7,7.5 \mathrm{~Hz}, 0.70 \mathrm{H}), 2.94(\mathrm{p}, J=7.3 \mathrm{~Hz}, 0.70 \mathrm{H}), 2.84-2.43(\mathrm{~m}$, $3.30 \mathrm{H}), 2.38-2.21(\mathrm{~m}, 1.0 \mathrm{H}), 2.15-2.03(\mathrm{~m}, 0.30 \mathrm{H})$.

${ }^{13}$ C NMR $\left(101 \mathrm{MHz}, \mathrm{CDCl}_{3}\right) \delta 173.1,173.0,172.9,172.7,141.5,140.8,138.7,138.2$, $128.4,128.3,128.1,127.6,126.6,126.3,115.6,115.4,59.0,58.1,52.9,52.8,51.5,50.9$, 48.0, 47.3, 42.5, 40.3, 39.0, 38.1.

DART-TOF LC/MS m/z calcd. for $\mathrm{C}_{17} \mathrm{H}_{21} \mathrm{O}_{4}\left[\mathrm{M}+\mathrm{H}^{+}\right] 289.1440$, found 289.1442.

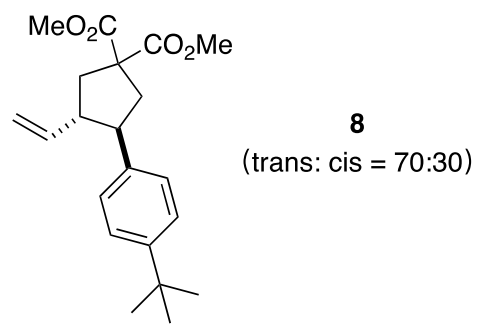

Prepared according to Procedure $\boldsymbol{C}$. Colorless oil (60.1 $\mathrm{mg}, 87 \%$ yield) as a mixture of diastereomers (trans : cis $=70: 30) . \mathrm{R}_{\mathrm{f}}=0.45(10 \%$ EtOAc in hexane).

${ }^{1} \mathbf{H}$ NMR $\left(400 \mathrm{MHz}, \mathrm{CDCl}_{3}\right) \delta 7.34-7.25(\mathrm{~m}, 2.0 \mathrm{H}), 7.15(\mathrm{~d}, J=8.3 \mathrm{~Hz}, 0.60 \mathrm{H}), 7.08(\mathrm{~d}$, $J=8.3 \mathrm{~Hz}, 1.40 \mathrm{H}), 5.73-5.60(\mathrm{~m}, 0.30 \mathrm{H}), 5.39(\mathrm{ddd}, J=17.1,10.3,7.9 \mathrm{~Hz}, 0.70 \mathrm{H})$, $4.99-4.80(\mathrm{~m}, 2.0 \mathrm{H}), 3.82-3.70(\mathrm{~m}, 6.0 \mathrm{H}), 3.41(\mathrm{dt}, J=10.5,7.4 \mathrm{~Hz}, 0.70 \mathrm{H}), 3.05-$ $2.96(\mathrm{~m}, 0.70 \mathrm{H}), 2.92-2.52(\mathrm{~m}, 3.30 \mathrm{H}), 2.46-2.27(\mathrm{~m}, 1.0 \mathrm{H}), 2.21-2.11(\mathrm{~m}, 0.30 \mathrm{H})$.

${ }^{13}$ C NMR $\left(101 \mathrm{MHz}, \mathrm{CDCl}_{3}\right) \delta 173.2,173.0,173.0,172.7,149.3,149.0,139.0,138.4$, $138.3,137.6,128.0,127.2,125.3,124.9,115.5,115.3,59.0,58.1,52.9,52.8,50.8,50.4$, 47.6, 47.2, 42.6, 40.3, 38.9, 38.2, 34.4, 34.4, 31.4 .

DART-TOF LC/MS m/z calcd. for $\mathrm{C}_{21} \mathrm{H}_{29} \mathrm{O}_{4}\left[\mathrm{M}+\mathrm{H}^{+}\right] 345.2066$, found 345.2067.

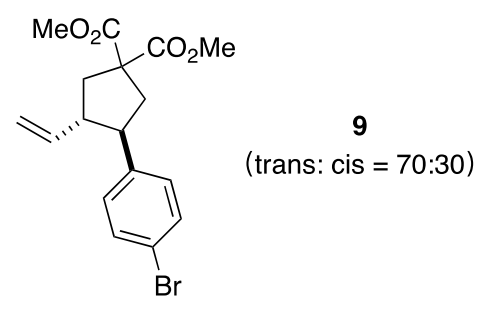

Prepared according to Procedure $\boldsymbol{C}$. Colorless oil (67.3 $\mathrm{mg}, 92 \%$ yield) as a mixture of diastereomers (trans : $\operatorname{cis}=70: 30) . \mathrm{R}_{\mathrm{f}}=0.40(10 \%$ EtOAc in hexane). 
${ }^{1} \mathbf{H}$ NMR $\left(400 \mathrm{MHz}, \mathrm{CDCl}_{3}\right) \delta 7.39(\mathrm{dd}, J=8.4,6.5 \mathrm{~Hz}, 2.0 \mathrm{H}), 7.09(\mathrm{~d}, J=8.5 \mathrm{~Hz}$, $0.60 \mathrm{H}), 7.04-6.98(\mathrm{~m}, 1.40 \mathrm{H}), 5.60$ (ddd, $J=17.3,10.3,7.1 \mathrm{~Hz}, 0.30 \mathrm{H}), 5.33$ (ddd, $J=$ $17.1,10.3,8.1 \mathrm{~Hz}, 0.70 \mathrm{H}), 4.94-4.79(\mathrm{~m}, 2.0 \mathrm{H}), 3.90-3.70(\mathrm{~m}, 6.0 \mathrm{H}), 3.39$ (dt, $J=$ 10.7, $7.5 \mathrm{~Hz}, 0.70 \mathrm{H}), 3.04-2.93(\mathrm{~m}, 0.70 \mathrm{H}), 2.87-2.47(\mathrm{~m}, 3.30 \mathrm{H}), 2.40-2.26(\mathrm{~m}$, $1.0 \mathrm{H}), 2.22-2.09(\mathrm{~m}, 0.30 \mathrm{H})$.

${ }^{13}$ C NMR $\left(101 \mathrm{MHz}, \mathrm{CDCl}_{3}\right) \delta 173.0,172.9,172.8,172.6,140.6,139.8,138.3,137.8$, $131.5,131.1,130.1,129.4,120.3,120.1,116.0,115.8,58.9,58.1,53.0,52.9,52.9,52.8$, 51.0, 50.9, 47.5, 47.3, 42.2, 40.3, 39.0, 38.1.

DART-TOF LC/MS m/z calcd. for $\mathrm{C}_{17} \mathrm{H}_{19} \mathrm{BrO}_{4}\left[\mathrm{M}+\mathrm{H}^{+}\right] 367.0545$, found 367.0541 .

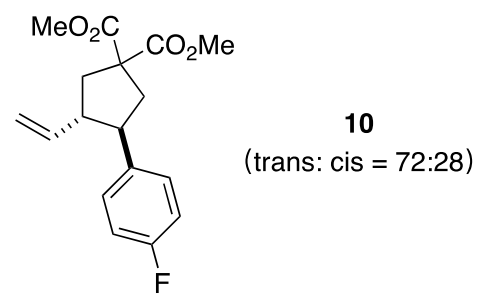

Prepared according to Procedure $\boldsymbol{C}$. Colorless oil (52.7 $\mathrm{mg}, 86 \%$ yield) as a mixture of diastereomers (trans : $\operatorname{cis}=72: 28) . \mathrm{R}_{\mathrm{f}}=0.35(10 \%$ EtOAc in hexane).

${ }^{1} \mathbf{H}$ NMR $\left(400 \mathrm{MHz}, \mathrm{CDCl}_{3}\right) \delta 7.21-6.90(\mathrm{~m}, 4.0 \mathrm{H}), 5.59(\mathrm{~d}, J=17.3 \mathrm{~Hz}, 0.28 \mathrm{H}), 5.34$ (ddd, $J=17.1,10.3,8.1 \mathrm{~Hz}, 0.72 \mathrm{H}), 4.94-4.77(\mathrm{~m}, 2.0 \mathrm{H}), 3.90-3.65(\mathrm{~m}, 6.0 \mathrm{H}), 3.41$ $(\mathrm{dt}, J=10.8,7.5 \mathrm{~Hz}, 0.72 \mathrm{H}), 3.05-2.93(\mathrm{~m}, 0.72 \mathrm{H}), 2.89-2.47(\mathrm{~m}, 2.28 \mathrm{H}), 2.40-2.26$ (m, $1.0 \mathrm{H}), 2.22-2.10(\mathrm{~m}, 0.28 \mathrm{H})$.

${ }^{13} \mathrm{C}$ NMR $\left(101 \mathrm{MHz}, \mathrm{CDCl}_{3}\right) \delta 173.0,172.9,172.8,172.6,162.8,162.6,160.4,160.2$, $138.5,138.0,137.2,137.1,136.4,136.4,129.7,129.6,129.0,128.9,115.8,115.6,115.3$, $115.1,114.9,114.7,58.9,58.0,52.9,52.8,52.8,51.1,50.7,47.4,47.3,42.4,40.2,38.9$, 38.3 .

${ }^{19}$ F NMR $\left(376 \mathrm{MHz}, \mathrm{CDCl}_{3}\right) \delta-116.6,-117.0$.

DART-TOF LC/MS m/z calcd. for $\mathrm{C}_{17} \mathrm{H}_{19} \mathrm{FO}_{4}\left[\mathrm{M}+\mathrm{H}^{+}\right] 307.1346$, found 307.1349.

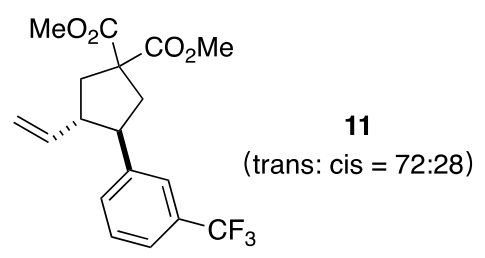

Prepared according to Procedure $\boldsymbol{C}$. Colorless oil (49.6 $\mathrm{mg}, 70 \%$ yield) as a mixture of diastereomers (trans : $\operatorname{cis}=72: 28) . \mathrm{R}_{\mathrm{f}}=0.40(10 \%$ EtOAc in hexane).

${ }^{1} \mathbf{H}$ NMR $\left(400 \mathrm{MHz} \mathrm{CDCl}_{3}\right) \delta 7.49-7.30(\mathrm{~m}, 4 \mathrm{H}), 5.62(\mathrm{ddd}, J=17.4,10.4,7.2 \mathrm{~Hz}$, $0.28 \mathrm{H}), 5.32(\mathrm{ddd}, J=17.1,10.3,8.2 \mathrm{~Hz}, 0.72 \mathrm{H}), 4.96-4.76(\mathrm{~m}, 2.0 \mathrm{H}), 3.90-3.65(\mathrm{~m}$, $6.0 \mathrm{H}), 3.55-3.42(\mathrm{~m}, 0.72 \mathrm{H}), 3.17-3.00(\mathrm{~m}, 0.72 \mathrm{H}), 2.98-2.86(\mathrm{~m}, 0.28 \mathrm{H}), 2.85-$ $2.52(\mathrm{~m}, 3.0 \mathrm{H}), 2.45-2.30(\mathrm{~m}, 1.0 \mathrm{H}), 2.25-2.12(\mathrm{~m}, 0.28 \mathrm{H})$.

${ }^{13} \mathrm{C}$ NMR $\left(101 \mathrm{MHz}, \mathrm{CDCl}_{3}\right) \delta 172.9,172.8,172.7,172.5,142.6,141.7,138.2,137.5$, $131.7,131.7,131.7,131.0,130.9,130.8,130.5,130.5,130.2,129.9,128.8,128.5,125.6$, $125.1,125.1,125.0,125.0,124.4,124.4,124.3,123.5,123.4,123.4,123.2,123.2,123.1$, 
123.1, 122.9, 116.2, 116.1, 58.9, 58.1, 53.0, 52.9, 52.8, 51.1, 51.0, 47.9, 47.3, 42.2, 40.3, $38.9,37.9$.

${ }^{19}$ F NMR $\left(376 \mathrm{MHz}, \mathrm{CDCl}_{3}\right) \delta-62.60,-62.61$.

DART-TOF LC/MS m/z calcd. for $\mathrm{C}_{18} \mathrm{H}_{19} \mathrm{~F}_{3} \mathrm{O}_{4}\left[\mathrm{M}+\mathrm{H}^{+}\right] 357.1314$, found 357.1314 .

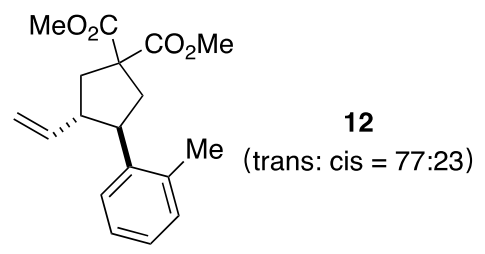

Prepared according to Procedure $\boldsymbol{C}$. Colorless oil (53.9 mg, $89 \%$ yield) as a mixture of diastereomers (trans : cis $=72: 28) . \mathrm{R}_{\mathrm{f}}=0.40(10 \%$ EtOAc in hexane $)$.

${ }^{1} \mathbf{H}$ NMR $\left(400 \mathrm{MHz}, \mathrm{CDCl}_{3}\right) \delta 7.24-7.06(\mathrm{~m}, 4.0 \mathrm{H}), 5.63(\mathrm{ddd}, J=17.4,10.4,7.2 \mathrm{~Hz}$, $0.23 \mathrm{H}), 5.31(\mathrm{ddd}, J=17.0,10.3,8.3 \mathrm{~Hz}, 0.77 \mathrm{H}), 4.97-4.70(\mathrm{~m}, 2.0 \mathrm{H}), 3.83-3.72(\mathrm{~m}$, $6.0 \mathrm{H}), 3.58(\mathrm{ddd}, J=11.0,8.3,7.1 \mathrm{~Hz}, 0.77 \mathrm{H}), 3.23-3.03(\mathrm{~m}, 1.0 \mathrm{H}), 2.95-2.55(\mathrm{~m}$, $3.0 \mathrm{H}), 2.39-2.27(\mathrm{~m}, 3.77 \mathrm{H}), 2.13(\mathrm{ddd}, J=13.8,11.3,2.4 \mathrm{~Hz}, 0.46 \mathrm{H})$.

${ }^{13} \mathrm{C}$ NMR $\left(101 \mathrm{MHz}, \mathrm{CDCl}_{3}\right) \delta 173.1,173.0,173.0,172.7,139.6,138.6,138.5,138.3$, $136.5,136.4,130.2,130.1,126.7,126.3,126.2,126.1,125.7,125.6,115.6,114.9,58.8$, 58.0, 52.9, 52.8, 50.0, 46.6, 45.0, 43.9, 42.1, 40.0, 39.1, 38.1, 20.1, 19.8 .

DART-TOF LC/MS m/z calcd. for $\mathrm{C}_{18} \mathrm{H}_{23} \mathrm{O}_{4}\left[\mathrm{M}+\mathrm{H}^{+}\right] 303.1596$, found 303.1595 .
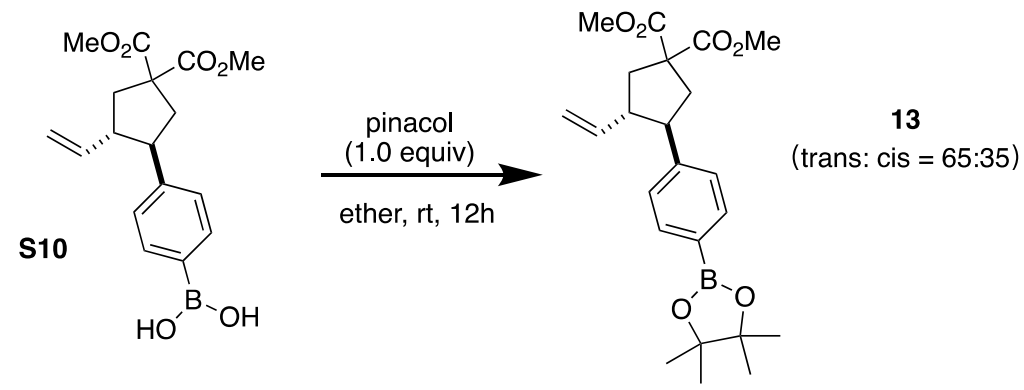

The boric acid product S10 was prepared according to Procedure $\boldsymbol{C}$. To a solution of crude S10 in anhydrous ether $(10.0 \mathrm{~mL})$, pinacol $(23.6 \mathrm{mg}, 0.2 \mathrm{mmol}, 1.0$ equiv) was added. The reaction was stirred at room temperature for $12 \mathrm{~h}$. The solvent was then removed under vacuum, and the residue was purified by silica gel chromatography to give $\mathbf{1 3}$ as a colorless oil $(69.7 \mathrm{mg}, 84 \%$ yield, trans $:$ cis $=65: 35) . \mathrm{R}_{\mathrm{f}}=0.45(10 \%$ EtOAc in hexane $)$.

${ }^{1} \mathbf{H}$ NMR $\left(400 \mathrm{MHz}, \mathrm{CDCl}_{3}\right) \delta 7.78-7.66(\mathrm{~m}, 2.0 \mathrm{H}), 7.24-7.20(\mathrm{~m}, 0.70 \mathrm{H}), 7.17-7.12$ $(\mathrm{m}, 1.30 \mathrm{H}), 5.62(\mathrm{ddd}, J=16.7,10.7,7.1 \mathrm{~Hz}, 0.35 \mathrm{H}), 5.34(\mathrm{ddd}, J=17.1,10.3,8.0 \mathrm{~Hz}$, $0.65 \mathrm{H}), 4.92-4.74(\mathrm{~m}, 2.0 \mathrm{H}), 3.95-3.65(\mathrm{~m}, 6.0 \mathrm{H}), 3.50-3.40(\mathrm{~m}, 0.65 \mathrm{H}), 3.10-2.98$ $(\mathrm{m}, 0.65 \mathrm{H}), 2.88(\mathrm{td}, J=11.1,7.6 \mathrm{~Hz}, 0.35 \mathrm{H}), 2.81-2.53(\mathrm{~m}, 3.0 \mathrm{H}), 2.44-2.30(\mathrm{~m}$, $1.0 \mathrm{H}), 2.22-2.12(\mathrm{~m}, 0.35 \mathrm{H}), 1.32(\mathrm{~s}, 12.0 \mathrm{H})$.

${ }^{13}$ C NMR $\left(101 \mathrm{MHz}, \mathrm{CDCl}_{3}\right) \delta 173.1,172.9,172.8,172.6,144.8,144.0,138.6,138.0$, 134.9 , 134.6, 127.8, 127.1, 115.7, 115.5, 83.7, 59.0, 58.1, 52.9, 52.8, 52.8, 51.7, 50.9, $48.2,47.3,42.3,40.3,39.0,37.9,24.9,24.9,24.8$.

DART-TOF LC/MS m/z calcd. for $\mathrm{C}_{23} \mathrm{H}_{32} \mathrm{BO}_{6}\left[\mathrm{M}+\mathrm{H}^{+}\right] 415.2292$, found 415.2292. 


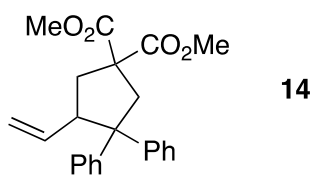

Prepared according to Procedure $\boldsymbol{C}$. Colorless oil ( $70.1 \mathrm{mg}, 96 \%$ yield). $\mathrm{R}_{\mathrm{f}}=0.40$ (10\% EtOAc in hexane).

${ }^{1} \mathbf{H}$ NMR $\left(400 \mathrm{MHz}, \mathrm{CDCl}_{3}\right) \delta 7.36-6.75(\mathrm{~m}, 10 \mathrm{H}), 5.37(\mathrm{ddd}, J=17.1,10.3,8.1 \mathrm{~Hz}$, $1 \mathrm{H}), 5.03(\mathrm{dd}, J=17.2,1.4 \mathrm{~Hz}, 1 \mathrm{H}), 4.92-4.81(\mathrm{~m}, 1 \mathrm{H}), 3.69(\mathrm{~s}, 3 \mathrm{H}), 3.56(\mathrm{dd}, J=8.6$, $6.4 \mathrm{~Hz}, 1 \mathrm{H}), 3.47$ (s, 3H), $3.44(\mathrm{~d}, J=14.7 \mathrm{~Hz}, 1 \mathrm{H}), 2.89$ (d, $J=14.7 \mathrm{~Hz}, 1 \mathrm{H}), 2.59$ (dd, $J$ $=13.8,6.4 \mathrm{~Hz}, 1 \mathrm{H}), 2.14(\mathrm{dd}, J=13.8,9.2 \mathrm{~Hz}, 1 \mathrm{H})$.

${ }^{13}$ C NMR $\left(101 \mathrm{MHz}, \mathrm{CDCl}_{3}\right) \delta 172.7,172.6,148.2,144.9,138.5,129.0,128.1,127.6$, $127.4,126.1,126.0,116.1,58.1,57.8,53.0,52.8,50.2,46.5,38.7$.

DART-TOF LC/MS m/z calcd. for $\mathrm{C}_{23} \mathrm{H}_{25} \mathrm{O}_{4}[\mathrm{M}+\mathrm{H}]^{+} 365.1753$, found 365.1758 .

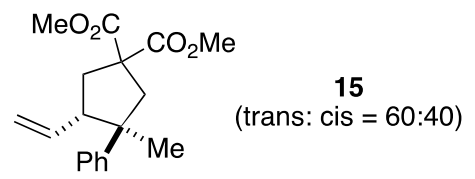

Prepared according to Procedure $\boldsymbol{C}$. Colorless oil (50.0 mg, $83 \%$ yield) as a mixture of diastereomers (trans : $c$ is $=60: 40) . \mathrm{R}_{\mathrm{f}}=0.40(10 \%$ EtOAc in hexane).

${ }^{1} \mathbf{H}$ NMR $\left(400 \mathrm{MHz}, \mathrm{CDCl}_{3}\right) \delta 7.39-7.01(\mathrm{~m}, 5.0 \mathrm{H}), 5.64(\mathrm{ddd}, J=17.4,10.5,7.0 \mathrm{~Hz}$, $0.60 \mathrm{H}), 5.11$ (ddd, $J=17.1,10.1,8.5 \mathrm{~Hz}, 0.40 \mathrm{H}), 4.99-4.72(\mathrm{~m}, 2.0 \mathrm{H}), 3.75-3.61(\mathrm{~m}$, $6.0 \mathrm{H}), 3.04-2.89(\mathrm{~m}, 1.0 \mathrm{H}), 2.72-2.58(\mathrm{~m}, 1.0 \mathrm{H}), 2.58-2.33(\mathrm{~m}, 2.60 \mathrm{H}), 2.22(\mathrm{dd}, J=$ $13.8,8.6 \mathrm{~Hz}, 0.40 \mathrm{H}), 1.33(\mathrm{~s}, 1.20 \mathrm{H}), 1.14(\mathrm{~d}, J=0.8 \mathrm{~Hz}, 1.80 \mathrm{H})$.

${ }^{13}$ C NMR $\left(101 \mathrm{MHz}, \mathrm{CDCl}_{3}\right) \delta 173.4,173.4,173.0,172.8,147.7,146.0,138.6,136.6$, $128.2,127.8,127.3,126.1,126.0,125.8,116.5,115.6,58.7,57.5,55.0,53.0,53.0,52.9$, $52.9,52.8,49.9,49.5,48.8,45.8,39.2,38.3,29.4,21.8$.

DART-TOF LC/MS m/z calcd. for $\mathrm{C}_{18} \mathrm{H}_{23} \mathrm{O}_{4}\left[\mathrm{M}+\mathrm{H}^{+}\right] 303.1596$, found 303.1602 .

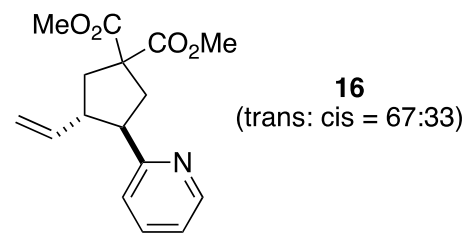

Prepared according to Procedure $\boldsymbol{C}$. Colorless oil (30.2 mg, 52\% yield) as a mixture of diastereomers (trans : $c$ is $=67: 33) . \mathrm{R}_{\mathrm{f}}=0.25(30 \%$ EtOAc in hexane).

${ }^{1} \mathbf{H}$ NMR $\left(400 \mathrm{MHz}, \mathrm{CDCl}_{3}\right) \delta 8.58-8.44(\mathrm{~m}, 1.0 \mathrm{H}), 7.63-7.48(\mathrm{~m}, 1.0 \mathrm{H}), 7.17-7.01$ (m, 2.0H), 5.67 (ddd, $J=17.1,10.4,7.5 \mathrm{~Hz}, 0.33 \mathrm{H}), 5.33(\mathrm{ddd}, J=17.1,10.2,8.3 \mathrm{~Hz}$, $0.67 \mathrm{H}), 4.94-4.69(\mathrm{~m}, 2.0 \mathrm{H}), 3.85-3.69(\mathrm{~m}, 6.0 \mathrm{H}), 3.56(\mathrm{dd}, J=8.0,7.9 \mathrm{~Hz}, 0.67 \mathrm{H})$, $3.18-2.68(\mathrm{~m}, 3.33 \mathrm{H}), 2.62-2.43(\mathrm{~m}, 1.67 \mathrm{H}), 2.13(\mathrm{dd}, J=13.7,10.7 \mathrm{~Hz}, 0.33 \mathrm{H})$.

${ }^{13}$ C NMR $\left(101 \mathrm{MHz}, \mathrm{CDCl}_{3}\right) \delta 173.5,173.1,172.6,172.6,160.8,160.8,149.4,148.9$, $138.9,138.1,136.2,135.7,123.3,123.0,121.7,121.3,115.5,115.4,59.6,58.4,53.3,52.9$, $52.8,52.8,52.7,50.3,50.2,47.7,40.9,40.1,39.1,37.8$.

DART-TOF LC/MS m/z calcd. for $\mathrm{C}_{16} \mathrm{H}_{20} \mathrm{NO}_{4}\left[\mathrm{M}+\mathrm{H}^{+}\right] 290.1392$, found 290.1393 . 


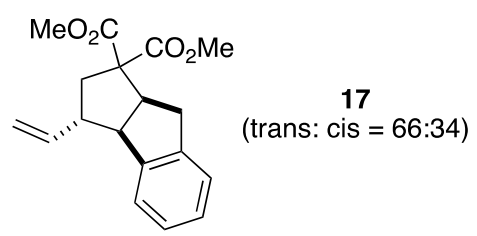

Prepared according to Procedure $\boldsymbol{C}$. Colorless oil (54.8 $\mathrm{mg}, 91 \%$ yield) as a mixture of diastereomers (trans : $\operatorname{cis}=66: 34) . \mathrm{R}_{\mathrm{f}}=0.40(10 \%$ EtOAc in hexane).

${ }^{1}$ H NMR $\left(400 \mathrm{MHz}, \mathrm{CDCl}_{3}\right) \delta 7.20-7.05(\mathrm{~m}, 4.0 \mathrm{H}), 5.95-5.79(\mathrm{~m}, 0.34 \mathrm{H}), 5.35$ (ddd, $J$ $=17.1,10.1,8.6 \mathrm{~Hz}, 0.66 \mathrm{H}), 5.12-4.88(\mathrm{~m}, 2.0 \mathrm{H}), 4.01-3.71(\mathrm{~m}, 6.68 \mathrm{H}), 3.66(\mathrm{~s}$, $1.0 \mathrm{H}), 3.49(\mathrm{dd}, J=8.5,7.1 \mathrm{~Hz}, 0.34 \mathrm{H}), 3.08(\mathrm{ddd}, J=16.7,9.7,7.9 \mathrm{~Hz}, 1.0 \mathrm{H}), 2.91-$ $2.72(\mathrm{~m}, 1.66 \mathrm{H}), 2.67-2.55(\mathrm{~m}, 0.66 \mathrm{H}), 2.28-1.94(\mathrm{~m}, 1.66 \mathrm{H})$.

${ }^{13} \mathrm{C}$ NMR $\left(101 \mathrm{MHz}, \mathrm{CDCl}_{3}\right) \delta 172.8,172.3,171.2,170.8,145.2,142.7,142.0,141.7$, $140.6,138.9,126.9,126.9,126.8,126.7,125.9$, 124.4, 124.1, 123.8, 115.2, 114.7, 64.1, $62.9,56.5,52.9,52.9,52.7,52.5,52.3,50.2,50.0,47.8,45.5,41.8,37.9,35.9,34.9$.

DART-TOF LC/MS m/z calcd. for $\mathrm{C}_{18} \mathrm{H}_{21} \mathrm{O}_{4}\left[\mathrm{M}+\mathrm{H}^{+}\right] 301.1440$, found 301.1442.

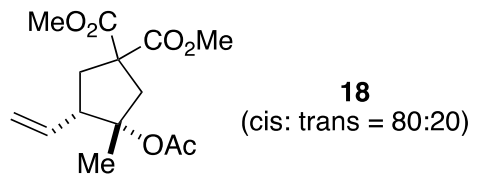

Prepared according to Procedure $\boldsymbol{C}$. Colorless oil (19.0 mg, 32\% yield) as a mixture of diastereomers (cis : trans $=80: 20) . \mathrm{R}_{\mathrm{f}}=0.35$ (30\% EtOAc in hexane).

${ }^{1} \mathbf{H}$ NMR $\left(400 \mathrm{MHz}, \mathrm{CDCl}_{3}\right) \delta 5.87-5.60(\mathrm{~m}, 1.0 \mathrm{H}), 5.17-5.05(\mathrm{~m}, 2.0 \mathrm{H}), 3.93-3.52$ $(\mathrm{m}, 6.0 \mathrm{H}), 3.40(\mathrm{~d}, J=15.4 \mathrm{~Hz}, 0.20 \mathrm{H}), 2.99-2.87(\mathrm{~m}, 0.80 \mathrm{H}), 2.83-2.57(\mathrm{~m}, 2.40 \mathrm{H})$, $2.52-2.18(\mathrm{~m}, 0.80 \mathrm{H}), 2.04(\mathrm{dd}, J=14.0,9.4 \mathrm{~Hz}, 0.80 \mathrm{H}), 1.95(\mathrm{~s}, 2.40 \mathrm{H}), 1.92$ (s, $0.60 \mathrm{H}), 1.52(\mathrm{~s}, 0.60 \mathrm{H}), 1.37(\mathrm{~s}, 2.40 \mathrm{H})$.

${ }^{13}$ C NMR (101 MHz, $\left.\mathrm{CDCl}_{3}\right) \delta 173.0,172.4,172.4,172.3,170.5,170.3,136.0,134.9$, $118.1,117.1,89.2,88.6,57.3,57.1,55.5,52.9,52.8,52.7,51.9,45.1,43.5,38.2,36.0$, 22.0, 22.0, 21.2, 20.0.

DART-TOF LC/MS m/z calcd. for $\mathrm{C}_{14} \mathrm{H}_{24} \mathrm{NO}_{6}\left[\mathrm{M}+\mathrm{NH}_{4}{ }^{+}\right] 302.1604$, found 302.1604 .

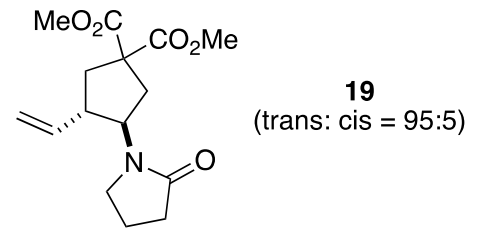

Prepared according to Procedure $\boldsymbol{C}$. Colorless oil (45.4 mg, 77\% yield) as a mixture of diastereomers (trans : $\operatorname{cis}=95: 5) . \mathrm{R}_{\mathrm{f}}=0.30$ (50\% EtOAc in hexane).

${ }^{1} \mathbf{H}$ NMR $\left(400 \mathrm{MHz}, \mathrm{CDCl}_{3}\right) \delta 5.77-5.62(\mathrm{~m}, 1 \mathrm{H}), 5.07-4.91(\mathrm{~m}, 2 \mathrm{H}), 4.59(\mathrm{q}, J=8.0$ $\mathrm{Hz}, 1 \mathrm{H}), 3.68(\mathrm{~d}, J=6.3 \mathrm{~Hz}, 6 \mathrm{H}), 3.32(\mathrm{dt}, J=9.3,7.0 \mathrm{~Hz}, 1 \mathrm{H}), 3.24(\mathrm{dt}, J=9.3,6.8 \mathrm{~Hz}$, $1 \mathrm{H}), 2.90$ (dddd, $J=9.7,6.9,2.8,1.5 \mathrm{~Hz}, 1 \mathrm{H}), 2.60$ (ddd, $J=14.4,8.1,1.0 \mathrm{~Hz}, 1 \mathrm{H}$ ), 2.49 $(\mathrm{ddd}, J=13.8,7.1,0.9 \mathrm{~Hz}, 1 \mathrm{H}), 2.36-2.23(\mathrm{~m}, 3 \mathrm{H}), 2.16(\mathrm{dd}, J=13.7,9.6 \mathrm{~Hz}, 1 \mathrm{H})$, $1.93-1.78(\mathrm{~m}, 2 \mathrm{H})$. 
${ }^{13} \mathrm{C}$ NMR $\left(101 \mathrm{MHz}, \mathrm{CDCl}_{3}\right) \delta 175.2,172.1,172.0,136.0,116.5,58.2,53.7,53.0,52.9$, 45.7, 45.3, 37.7, 35.5, 31.1, 18.3.

DART-TOF LC/MS m/z calcd. for $\mathrm{C}_{15} \mathrm{H}_{23} \mathrm{NO}_{5}[\mathrm{M}+\mathrm{H}]^{+} 296.1498$, found 296.1495 .

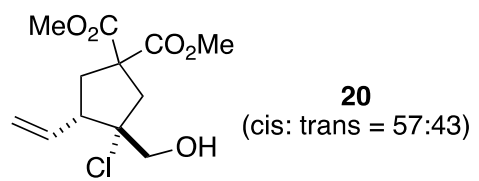

Prepared according to Procedure $\boldsymbol{C}$. Colorless oil (28.3 mg, $51 \%$ yield) as a mixture of diastereomers (cis : trans $=57: 43)$. $\mathrm{R}_{\mathrm{f}}=0.35(50 \%$ EtOAc in hexane).

${ }^{1} \mathbf{H}$ NMR $\left(400 \mathrm{MHz}, \mathrm{CDCl}_{3}\right) \delta 5.82-5.66(\mathrm{~m}, 1.0 \mathrm{H}), 5.23-5.00(\mathrm{~m}, 2.0 \mathrm{H}), 3.79-3.48$ (m, $8.0 \mathrm{H}), 3.14-2.53(\mathrm{~m}, 4.0 \mathrm{H}), 2.38(\mathrm{dd}, J=13.5,7.0 \mathrm{~Hz}, 0.43 \mathrm{H}), 2.22-2.06(\mathrm{~m}$, $1.57 \mathrm{H})$.

${ }^{13} \mathrm{C}$ NMR $\left(101 \mathrm{MHz}, \mathrm{CDCl}_{3}\right) \delta$ 172.7, 172.5, 171.9, 171.6, 135.4, 134.2, 118.6, 118.2, 77.4, 77.0, 76.7, 67.8, 67.0, 57.4, 57.0, 54.9, 53.2, 53.2, 53.1, 53.0, 50.6, 46.5, 44.9, 38.2, 37.6.

DART-TOF LC/MS m/z calcd. for $\mathrm{C}_{12} \mathrm{H}_{18} \mathrm{ClO}_{5}\left[\mathrm{M}+\mathrm{H}^{+}\right] 277.0843$, found 277.0846.

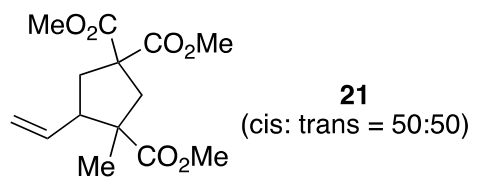

Prepared according to Procedure $\boldsymbol{C}$. Colorless oil ( $48.6 \mathrm{mg}, 85 \%$ yield) as a mixture of diastereomers (cis : trans $=50: 50) . \mathrm{R}_{\mathrm{f}}=0.40(20 \%$ EtOAc in hexane $)$.

${ }^{1} \mathbf{H}$ NMR $\left(400 \mathrm{MHz}, \mathrm{CDCl}_{3}\right) \delta 5.85-5.66(\mathrm{~m}, 0.50 \mathrm{H}), 5.66-5.55(\mathrm{~m}, 0.50 \mathrm{H}), 5.12-$ $4.94(\mathrm{~m}, 2 \mathrm{H}), 3.82-3.48(\mathrm{~m}, 9.0 \mathrm{H}), 3.12-2.98(\mathrm{~m}, 0.50 \mathrm{H}), 2.91(\mathrm{~d}, J=14.4 \mathrm{~Hz}, 0.50 \mathrm{H})$, $2.82(\mathrm{~d}, J=14.3 \mathrm{~Hz}, 0.50 \mathrm{H}), 2.57-2.44(\mathrm{~m}, 1.50 \mathrm{H}), 2.40-2.29(\mathrm{~m}, 1.0 \mathrm{H}), 2.28-2.11$ $(\mathrm{m}, 1.0 \mathrm{H}), 1.26(\mathrm{~s}, 1.50 \mathrm{H}), 1.07(\mathrm{~s}, 1.50 \mathrm{H})$.

${ }^{13} \mathrm{C}$ NMR $\left(101 \mathrm{MHz}, \mathrm{CDCl}_{3}\right) \delta 176.5,175.6,173.5,172.5,172.1,136.0,135.7,117.1$, $116.8,58.7,57.8,54.6,53.0,53.0,52.9,52.9,52.7,52.1,51.9,51.5,49.7,44.8,44.7,38.9$, 37.5, 22.7, 18.9.

DART-TOF LC/MS m/z calcd. for $\mathrm{C}_{14} \mathrm{H}_{21} \mathrm{O}_{6}\left[\mathrm{M}+\mathrm{H}^{+}\right] 285.1338$, found 285.1334 .

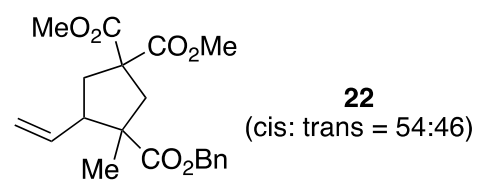

Prepared according to Procedure $\boldsymbol{C}$. Colorless oil (64.7 mg, 90\% yield) as a mixture of diastereomers (cis : trans $=54: 46) . \mathrm{R}_{\mathrm{f}}=0.40(50 \%$ EtOAc in hexane $)$.

${ }^{1} \mathbf{H}$ NMR $\left(400 \mathrm{MHz}, \mathrm{CDCl}_{3}\right) \delta 7.39-7.28(\mathrm{~m}, 5 \mathrm{H}), 5.74(\mathrm{ddd}, J=17.4,10.5,7.2 \mathrm{~Hz}$, $0.46 \mathrm{H}), 5.65-5.53(\mathrm{~m}, 0.54 \mathrm{H}), 5.16-4.94(\mathrm{~m}, 4.0 \mathrm{H}), 3.81-3.55(\mathrm{~m}, 6.0 \mathrm{H}), 3.15-3.04$ $(\mathrm{m}, 0.46 \mathrm{H}), 2.94(\mathrm{dd}, J=32.8,14.4 \mathrm{~Hz}, 1.0 \mathrm{H}), 2.62-2.48(\mathrm{~m}, 1.54 \mathrm{H}), 2.44-2.14(\mathrm{~m}$, $2.0 \mathrm{H}), 1.32(\mathrm{~s}, 1.62 \mathrm{H}), 1.13(\mathrm{~s}, 1.38 \mathrm{H})$. 
${ }^{13} \mathrm{C}$ NMR $\left(101 \mathrm{MHz}, \mathrm{CDCl}_{3}\right) \delta 175.9,175.1,173.5,172.5,172.4,171.9,135.9,135.8$, 135.6, 128.5, 128.5, 128.4, 128.2, 128.1, 127.9, 117.2, 117.0, 66.6, 66.4, 58.7, 57.9, 54.7, 53.0, 53.0, 52.9, 52.9, 52.7, 51.9, 49.8, 44.8, 44.7, 38.9, 37.6, 22.9, 19.1.

DART-TOF LC/MS m/z calcd. for $\mathrm{C}_{20} \mathrm{H}_{25} \mathrm{O}_{6}\left[\mathrm{M}+\mathrm{H}^{+}\right] 361.1651$, found 361.1652.

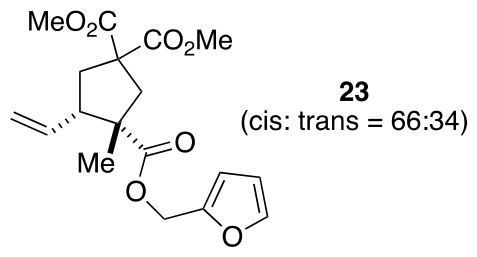

Prepared according to Procedure $\boldsymbol{C}$. Colorless oil (59.6 mg, 85\% yield) as a mixture of diastereomers (cis : trans $=66: 34) . \mathrm{R}_{\mathrm{f}}=0.40$ (20\% EtOAc in hexane).

${ }^{1} \mathbf{H}$ NMR $\left(400 \mathrm{MHz}, \mathrm{CDCl}_{3}\right) \delta 7.39(\mathrm{dd}, J=1.9,0.8 \mathrm{~Hz}, 1.0 \mathrm{H}), 6.39-6.29(\mathrm{~m}, 2.0 \mathrm{H})$, $5.70(\mathrm{ddd}, J=17.4,10.5,7.2 \mathrm{~Hz}, 0.34 \mathrm{H}), 5.60-5.48(\mathrm{~m}, 0.66 \mathrm{H}), 5.10-4.91(\mathrm{~m}, 4.0 \mathrm{H})$, $3.70(\mathrm{dd}, J=8.2,1.9 \mathrm{~Hz}, 6.0 \mathrm{H}), 3.10-3.00(\mathrm{~m}, 0.34 \mathrm{H}), 2.95(\mathrm{~d}, J=14.4 \mathrm{~Hz}, 0.66 \mathrm{H})$, $2.84(\mathrm{~d}, J=14.3 \mathrm{~Hz}, 0.34 \mathrm{H}), 2.60-2.44(\mathrm{~m}, 1.66 \mathrm{H}), 2.39-2.30(\mathrm{~m}, 1.0 \mathrm{H}), 2.27-2.12$ $(\mathrm{m}, 1.0 \mathrm{H}), 1.27(\mathrm{~s}, 1.98 \mathrm{H}), 1.08(\mathrm{~s}, 1.02 \mathrm{H})$.

${ }^{13} \mathrm{C}$ NMR $\left(101 \mathrm{MHz} \mathrm{CDCl}_{3}\right) \delta 175.7,174.8,173.5,172.4,172.4,171.9,149.5,149.4$, 143.1, 143.0, 135.8, 135.5, 117.1, 117.0, 110.6, 110.5, 110.4, 58.7, 58.4, 58.0, 57.9, 54.7, 53.0, 53.0, 52.9, 52.7, 52.0, 49.8, 44.7, 44.6, 38.9, 37.5, 22.8, 18.9.

DART-TOF LC/MS m/z calcd. for $\mathrm{C}_{18} \mathrm{H}_{23} \mathrm{O}_{7}\left[\mathrm{M}+\mathrm{H}^{+}\right] 351.1444$, found 351.1449.

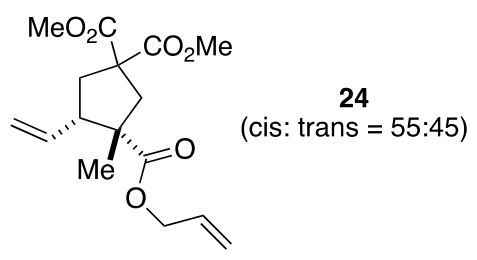

Prepared according to Procedure $\boldsymbol{C}$. Colorless oil (50.7 $\mathrm{mg}, 82 \%$ yield) as a mixture of diastereomers (cis : trans $=55: 45) . \mathrm{R}_{\mathrm{f}}=0.45$ (20\% EtOAc in hexane).

${ }^{1} \mathbf{H}$ NMR $\left(400 \mathrm{MHz}, \mathrm{CDCl}_{3}\right) \delta 6.12-5.48(\mathrm{~m}, 1.0 \mathrm{H}), 5.40-4.94(\mathrm{~m}, 4.0 \mathrm{H}), 4.59-4.45$ $(\mathrm{m}, 2.0 \mathrm{H}), 3.78-3.63(\mathrm{~m}, 6.0 \mathrm{H}), 3.14-3.02(\mathrm{~m}, 0.45 \mathrm{H}), 2.94(\mathrm{~d}, J=14.4 \mathrm{~Hz}, 0.55 \mathrm{H})$, $2.86(\mathrm{~d}, J=14.3 \mathrm{~Hz}, 0.45 \mathrm{H}), 2.61-2.46(\mathrm{~m}, 1.55 \mathrm{H}), 2.42-2.30(\mathrm{~m}, 1.0 \mathrm{H}), 2.29-2.11$ $(\mathrm{m}, 1.0 \mathrm{H}), 1.29(\mathrm{~s}, 1.65 \mathrm{H}), 1.10(\mathrm{~s}, 1.35 \mathrm{H})$.

${ }^{13} \mathrm{C}$ NMR $\left(101 \mathrm{MHz}, \mathrm{CDCl}_{3}\right) \delta 175.7,174.8,173.5,172.4,172.4,172.0,136.0,135.7$, 132.1, 118.2, 118.0, 117.2, 116.9, 65.4, 65.2, 58.7, 57.9, 54.6, 53.0, 52.9, 52.9, 52.7, 51.9, 49.7, 44.8, 44.7, 39.0, 37.5, 22.8, 19.0 .

DART-TOF LC/MS m/z calcd. for $\mathrm{C}_{16} \mathrm{H}_{23} \mathrm{O}_{6}\left[\mathrm{M}+\mathrm{H}^{+}\right] 311.1495$, found 311.1491 .

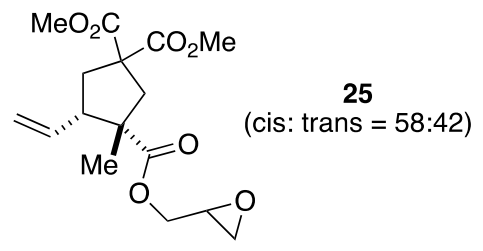


Prepared according to Procedure $\boldsymbol{C}$. Colorless oil (58.6 mg, 90\% yield) as a mixture of diastereomers (cis : trans $=58: 42) . \mathrm{R}_{\mathrm{f}}=0.30$ (20\% EtOAc in hexane).

${ }^{1} \mathbf{H}$ NMR $\left(400 \mathrm{MHz}, \mathrm{CDCl}_{3}\right) \delta 5.81-5.55$ (m, 1.0H), $5.13-4.99$ (m, 2.0H), 4.39 (ddd, $J$ $=12.3,4.6,3.0 \mathrm{~Hz}, 0.42 \mathrm{H}), 4.31(\mathrm{dd}, J=12.2,3.1 \mathrm{~Hz}, 0.58 \mathrm{H}), 3.90(\mathrm{ddd}, J=12.3,6.1$, $1.5 \mathrm{~Hz}, 0.42 \mathrm{H}), 3.81(\mathrm{ddd}, J=12.3,6.4,0.9 \mathrm{~Hz}, 0.58 \mathrm{H}), 3.74-3.67(\mathrm{~m}, 6.0 \mathrm{H}), 3.21-$ $2.73(\mathrm{~m}, 3.40 \mathrm{H}), 2.67-2.46(\mathrm{~m}, 2.60 \mathrm{H}), 2.42-2.13(\mathrm{~m}, 2.0 \mathrm{H}), 1.28(\mathrm{~s}, 1.74 \mathrm{H}), 1.09$ (s, $1.26 \mathrm{H})$.

${ }^{13} \mathrm{C}$ NMR $\left(101 \mathrm{MHz}, \mathrm{CDCl}_{3}\right) \delta 175.8,175.7,174.9,174.8,173.4,172.4,172.0,171.9$, 135.8, 135.6, 117.3, 117.1, 117.0, 65.3, 65.2, 65.1, 65.1, 58.6, 57.9, 54.5, 54.5, 53.1, 53.0, $52.9,52.9,52.8,52.7,52.0,52.0,49.7,49.2,49.1,49.1,44.8,44.7,44.6,44.6,44.4,44.4$, $38.8,38.8,37.5,22.8,22.8,19.1,19.0$.

DART-TOF LC/MS m/z calcd. for $\mathrm{C}_{16} \mathrm{H}_{26} \mathrm{NO}_{7}\left[\mathrm{M}+\mathrm{NH}_{4}{ }^{+}\right] 344.1709$, found 344.1705.

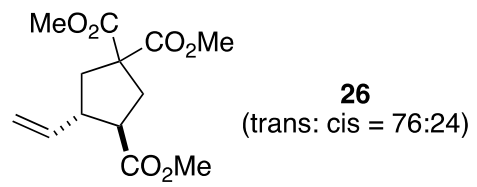

Prepared according to Procedure $\boldsymbol{C}$. Colorless oil (39.8 $\mathrm{mg}, 74 \%$ yield) as a mixture of diastereomers (trans : $\operatorname{cis}=76: 24) . \mathrm{R}_{\mathrm{f}}=0.30$ (30\% EtOAc in hexane).

${ }^{1}$ H NMR (400 MHz, $\left.\mathrm{CDCl}_{3}\right) \delta 5.82-5.56(\mathrm{~m}, 1.0 \mathrm{H}), 5.16-4.90(\mathrm{~m}, 2.0 \mathrm{H}), 3.89-3.52$ $(\mathrm{m}, 9.0 \mathrm{H}), 3.17-2.79(\mathrm{~m}, 1.76 \mathrm{H}), 2.74-2.31(\mathrm{~m}, 4.0 \mathrm{H}), 1.97(\mathrm{dd}, J=13.6,10.8 \mathrm{~Hz}$, $0.24 \mathrm{H})$.

${ }^{13} \mathrm{C}$ NMR $\left(101 \mathrm{MHz}, \mathrm{CDCl}_{3}\right) \delta 174.0,173.6,172.9,172.3,172.0,171.8,138.3,136.6$, $116.5,115.9,59.3,58.7,52.9,52.9,52.8,51.8,51.5,49.5,48.0,47.5,45.8,39.9,38.6$, 37.4, 35.9 .

DART-TOF LC/MS m/z calcd. for $\mathrm{C}_{13} \mathrm{H}_{19} \mathrm{O}_{6}\left[\mathrm{M}+\mathrm{H}^{+}\right] 271.1182$, found 271.1180.

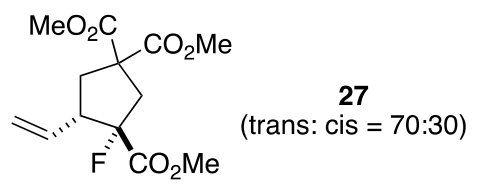

Prepared according to Procedure $\boldsymbol{C}$. Colorless oil (33.3 $\mathrm{mg}, 58 \%$ yield) as a mixture of diastereomers (trans : cis $=70: 30) . \mathrm{R}_{\mathrm{f}}=0.35$ (30\% EtOAc in hexane).

${ }^{1} \mathbf{H}$ NMR $\left(400 \mathrm{MHz}, \mathrm{CDCl}_{3}\right) \delta 5.84-5.49(\mathrm{~m}, 1.0 \mathrm{H}), 5.24-4.96(\mathrm{~m}, 2.0 \mathrm{H}), 4.01-3.55$ $(\mathrm{m}, 9.0 \mathrm{H}), 3.28-2.69(\mathrm{~m}, 3.70 \mathrm{H}), 2.57(\mathrm{dd}, J=13.6,7.7 \mathrm{~Hz}, 0.30 \mathrm{H}), 2.51-2.42(\mathrm{~m}$, $0.30 \mathrm{H}), 2.24(\mathrm{dd}, J=13.7,10.0 \mathrm{~Hz}, 0.70 \mathrm{H})$.

${ }^{13} \mathrm{C}$ NMR $\left(101 \mathrm{MHz} \mathrm{CDCl}_{3}\right) \delta 171.9,171.5,171.1,171.0,169.9,169.8,169.6,169.6$, 133.8, 133.7, 132.2, 132.1, 119.1, 118.6, 103.7, 103.1, 101.8, 101.1, 57.9, 57.9, 57.8, 53.2, 53.2, 53.1, 53.0, 52.8, 52.7, 52.4, 52.2, 52.0, 44.1, 43.9, 42.8, 42.6, 37.6, 37.6, 37.5.

${ }^{19}$ F NMR $\left(376 \mathrm{MHz}, \mathrm{CDCl}_{3}\right) \delta-149.0,-170.0$.

DART-TOF LC/MS m/z calcd. for $\mathrm{C}_{13} \mathrm{H}_{18} \mathrm{FO}_{6}\left[\mathrm{M}+\mathrm{H}^{+}\right] 289.1087$, found 289.1086. 


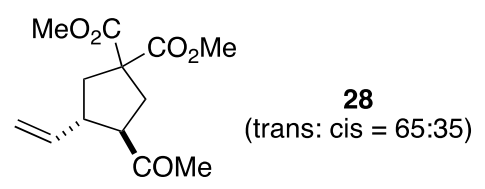

Prepared according to Procedure $\boldsymbol{C}$. Colorless oil (37.0 mg, 73\% yield) as a mixture of diastereomers (trans : $\operatorname{cis}=65: 35) . \mathrm{R}_{\mathrm{f}}=0.30$ (30\% EtOAc in hexane).

${ }^{1} \mathbf{H}$ NMR $\left(400 \mathrm{MHz}, \mathrm{CDCl}_{3}\right) \delta 5.71(\mathrm{ddd}, J=17.0,10.2,7.6 \mathrm{~Hz}, 0.35 \mathrm{H}), 5.59$ (ddd, $J=$ $17.0,10.1,9.2 \mathrm{~Hz}, 0.65 \mathrm{H}), 5.15-4.96(\mathrm{~m}, 2.0 \mathrm{H}), 3.94-3.50(\mathrm{~m}, 6.0 \mathrm{H}), 3.22(\mathrm{td}, J=8.6$, $7.1 \mathrm{~Hz}, 0.65 \mathrm{H}), 3.08-2.96(\mathrm{~m}, 0.65 \mathrm{H}), 2.86-2.72(\mathrm{~m}, 0.65 \mathrm{H}), 2.67-2.50(\mathrm{~m}, 1.35 \mathrm{H})$, $2.48-2.22(\mathrm{~m}, 2.35 \mathrm{H}), 2.12(\mathrm{~s}, 1.05 \mathrm{H}), 2.08(\mathrm{~s}, 1.95 \mathrm{H}), 2.02-1.89(\mathrm{~m}, 0.35 \mathrm{H})$.

${ }^{13}$ C NMR $\left(101 \mathrm{MHz} \mathrm{CDCl}_{3}\right) \delta$ 208.3, 208.2, 172.9, 172.4, 172.0, 171.9, 139.1, 137.0, $116.6,116.1,59.0,58.7,57.2,55.4,52.9,52.9$, 52.8, 47.0, 45.8, 40.3, 39.4, 36.4, 34.8, $31.0,30.0$.

DART-TOF LC/MS m/z calcd. for $\mathrm{C}_{34} \mathrm{H}_{19} \mathrm{O}_{5}\left[\mathrm{M}+\mathrm{H}^{+}\right] 255.1232$, found 255.1233.

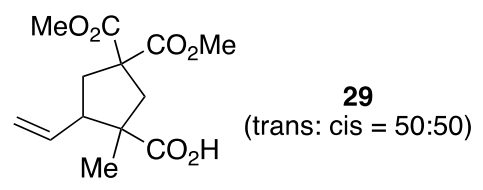

Prepared according to Procedure $\boldsymbol{C}$. Colorless oil (48.7 $\mathrm{mg}, 90 \%$ yield) as a mixture of diastereomers (trans : cis $=50: 50) . \mathrm{R}_{\mathrm{f}}=0.40(10 \% \mathrm{MeOH}$ in $\mathrm{DCM})$.

${ }^{1} \mathbf{H}$ NMR $\left(400 \mathrm{MHz}, \mathrm{CDCl}_{3}\right) \delta 11.63$ (brs, 1.0H), $5.93-5.54(\mathrm{~m}, 1.0 \mathrm{H}), 5.19-4.98$ (m, $2.0 \mathrm{H}), 3.88-3.60(\mathrm{~m}, 6.0 \mathrm{H}), 3.13-3.03(\mathrm{~m}, 0.50 \mathrm{H}), 2.95(\mathrm{~d}, J=14.4 \mathrm{~Hz}, 0.50 \mathrm{H}), 2.88$ $(\mathrm{d}, J=14.4 \mathrm{~Hz}, 0.50 \mathrm{H}), 2.55(\mathrm{ddd}, J=12.4,9.2,4.8 \mathrm{~Hz}, 1.50 \mathrm{H}), 2.44-2.33(\mathrm{~m}, 1.0 \mathrm{H})$, $2.31-2.13(\mathrm{~m}, 1.0 \mathrm{H}), 1.28(\mathrm{~s}, 1.50 \mathrm{H}), 1.10(\mathrm{~s}, 1.50 \mathrm{H})$.

${ }^{13}$ C NMR $\left(101 \mathrm{MHz}, \mathrm{CDCl}_{3}\right) \delta 182.7,181.9,173.4,172.4,172.4,171.9,135.6,135.4$, 117.7, 117.2, 58.8, 57.9, 54.7, 53.0, 53.0, 53.0, 52.8, 52.8, 51.7, 49.6, 44.8, 44.6, 39.0, 37.6, 22.6, 18.8 .

DART-TOF LC/MS m/z calcd. for $\mathrm{C}_{13} \mathrm{H}_{20} \mathrm{O}_{6}\left[\mathrm{M}+\mathrm{H}^{+}\right] 271.1182$, found 271.1185.

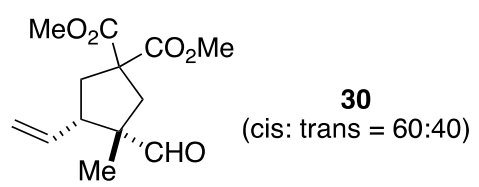

Prepared according to Procedure $\boldsymbol{C}$. Colorless oil (43.8 $\mathrm{mg}, 86 \%$ yield) as a mixture of diastereomers (cis : trans $=60: 40) . \mathrm{R}_{\mathrm{f}}=0.40(20 \%$ EtOAc in hexane).

${ }^{1} \mathbf{H}$ NMR $\left(400 \mathrm{MHz}, \mathrm{CDCl}_{3}\right) \delta 9.48(\mathrm{~s}, 0.40 \mathrm{H}), 9.43(\mathrm{~s}, 0.60 \mathrm{H}), 5.76(\mathrm{ddd}, J=17.1,10.3$, $8.0 \mathrm{~Hz}, 0.40 \mathrm{H}), 5.60$ (ddd, $J=17.1,10.5,7.5 \mathrm{~Hz}, 0.60 \mathrm{H}), 5.22-4.97$ (m, 2.0H), $3.93-$ $3.55(\mathrm{~m}, 6.0 \mathrm{H}), 2.89(\mathrm{dd}, J=11.7,0.9 \mathrm{~Hz}, 0.60 \mathrm{H}), 2.78(\mathrm{dd}, J=25.8,14.5 \mathrm{~Hz}, 1.0 \mathrm{H})$, $2.52(\mathrm{dd}, J=13.6,6.9 \mathrm{~Hz}, 1.0 \mathrm{H}), 2.45-2.37(\mathrm{~m}, 0.40 \mathrm{H}), 2.36-2.10(\mathrm{~m}, 2.0 \mathrm{H}), 1.24(\mathrm{~s}$, $1.20 \mathrm{H}), 1.00(\mathrm{~s}, 1.80 \mathrm{H})$.

${ }^{13} \mathrm{C}$ NMR (101 MHz, $\left.\mathrm{CDCl}_{3}\right) \delta$ 204.7, 203.1, 172.9, 172.4, 172.1, 171.9, 135.0, 134.4, $118.0,117.6,58.4,58.1,56.2$, 56.1, 53.8, 53.1, 53.0, 53.0, 52.9, 47.5, 41.8, 40.5, 38.9, $38.0,19.8,15.8$. 
DART-TOF LC/MS m/z calcd. for $\mathrm{C}_{13} \mathrm{H}_{19} \mathrm{O}_{5}\left[\mathrm{M}+\mathrm{H}^{+}\right] 255.1232$, found 255.1231.

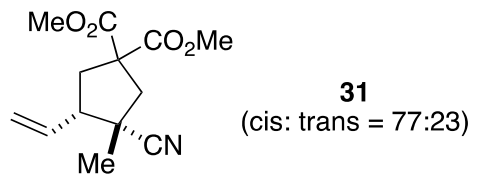

Prepared according to Procedure $\boldsymbol{C}$. Colorless oil (37.8 $\mathrm{mg}, 75 \%$ yield) as a mixture of diastereomers (trans : $\operatorname{cis}=77: 23) . \mathrm{R}_{\mathrm{f}}=0.30$ (30\% EtOAc in hexane).

${ }^{1} \mathbf{H}$ NMR $\left(400 \mathrm{MHz}, \mathrm{CDCl}_{3}\right) \delta 5.90-5.62(\mathrm{~m}, 1.0 \mathrm{H}), 5.31-5.14(\mathrm{~m}, 2.0 \mathrm{H}), 3.96-3.53$ $(\mathrm{m}, 6.0 \mathrm{H}), 3.05-2.90(\mathrm{~m}, 1.0 \mathrm{H}), 2.83(\mathrm{~d}, J=14.4 \mathrm{~Hz}, 0.77 \mathrm{H}), 2.62-2.48(\mathrm{~m}, 1.77 \mathrm{H})$, $2.48-2.31(\mathrm{~m}, 0.46 \mathrm{H}), 2.30-2.14(\mathrm{~m}, 1.0 \mathrm{H}), 1.38(\mathrm{~s}, 0.69 \mathrm{H}), 1.20(\mathrm{~s}, 2.31 \mathrm{H})$.

${ }^{13} \mathrm{C}$ NMR $\left(101 \mathrm{MHz}, \mathrm{CDCl}_{3}\right) \delta 172.2,171.7,171.6,171.0,134.4,133.3,123.8,122.0$, $119.7,119.2$, 57.7, 57.5, 53.9, 53.3, 53.2, 53.2, 53.2, 51.5, 45.7, 45.2, 43.4, 40.3, 39.2, 36.6, 22.8, 19.7.

DART-TOF LC/MS m/z calcd. for $\mathrm{C}_{13} \mathrm{H}_{18} \mathrm{NO}_{4}\left[\mathrm{M}+\mathrm{H}^{+}\right] 252.1236$, found 252.1232.

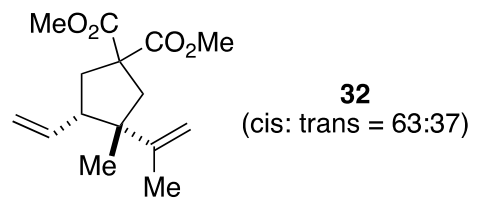

Prepared according to Procedure $\boldsymbol{C}$. Colorless oil (27.1 mg, 51\% yield) as a mixture of diastereomers (cis : trans $=63: 37) . \mathrm{R}_{\mathrm{f}}=0.40(10 \%$ EtOAc in hexane).

${ }^{1} \mathbf{H}$ NMR $\left(400 \mathrm{MHz}, \mathrm{CDCl}_{3}\right) \delta 5.78-5.57(\mathrm{~m}, 1.0 \mathrm{H}), 5.08-4.89(\mathrm{~m}, 2.0 \mathrm{H}), 4.85-4.69$ $(\mathrm{m}, 2.0 \mathrm{H}), 4.02-3.52(\mathrm{~m}, 6.0 \mathrm{H}), 2.88-2.76(\mathrm{~m}, 1.0 \mathrm{H}), 2.71(\mathrm{dd}, J=14.1,6.8 \mathrm{~Hz}$, $0.37 \mathrm{H}), 2.51-2.20(\mathrm{~m}, 3.63 \mathrm{H}), 1.77(\mathrm{~d}, J=1.0 \mathrm{~Hz}, 1.89 \mathrm{H}), 1.69(\mathrm{~d}, J=1.4 \mathrm{~Hz}, 1.11 \mathrm{H})$, $1.11(\mathrm{~s}, 1.11 \mathrm{H}), 0.96(\mathrm{~s}, 1.89 \mathrm{H})$.

${ }^{13} \mathrm{C}$ NMR $\left(101 \mathrm{MHz}, \mathrm{CDCl}_{3}\right) \delta 173.5,173.4,173.1,173.0,149.7,149.6,139.0,137.0$, 116.0, 114.7, 111.0, 110.5, 58.5, 57.4, 53.3, 52.9, 52.9, 52.8, 51.3, 49.9, 49.0, 46.2, 44.0, 38.9, 38.0, 26.2, 21.0, 20.1, 20.0.

DART-TOF LC/MS m/z calcd. for $\mathrm{C}_{15} \mathrm{H}_{26} \mathrm{NO}_{4}\left[\mathrm{M}+\mathrm{NH}_{4}{ }^{+}\right] 284.1862$, found 284.1868.

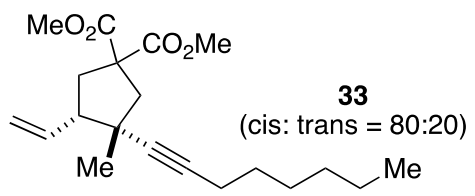

Prepared according to Procedure $\boldsymbol{C}$. Colorless oil (33.6 $\mathrm{mg}, 50 \%$ yield) as a mixture of diastereomers (cis : trans $=80: 20) . \mathrm{R}_{\mathrm{f}}=0.45(10 \%$ EtOAc in hexane).

${ }^{1} \mathbf{H}$ NMR $\left(400 \mathrm{MHz}, \mathrm{CDCl}_{3}\right) \delta 5.88-2.42(\mathrm{~m}, 1.0 \mathrm{H}), 5.24-4.88(\mathrm{~m}, 2.0 \mathrm{H}), 3.93-3.43$ $(\mathrm{m}, 6.0 \mathrm{H}), 2.81-2.63(\mathrm{~m}, 1.0 \mathrm{H}), 2.60-2.42(\mathrm{~m}, 2.60 \mathrm{H}), 2.33-2.02(\mathrm{~m}, 3.40 \mathrm{H}), 1.50-$ $1.21(\mathrm{~m}, 8.0 \mathrm{H}), 1.19(\mathrm{~s}, 0.60 \mathrm{H}), 0.99(\mathrm{~s}, 2.40 \mathrm{H}), 0.90-0.84(\mathrm{~m}, 3.0 \mathrm{H})$.

${ }^{13} \mathrm{C}$ NMR $\left(101 \mathrm{MHz}, \mathrm{CDCl}_{3}\right) \delta 173.5,172.8,172.8,172.2,137.6,136.2,116.8,116.4$, $85.8,83.9,82.5,80.9,57.8,57.7,55.0,53.4,52.9,52.8,52.8,52.6,48.9,48.0,42.5,40.8$, $39.4,36.8,31.3,31.3,29.0,28.9,28.4,28.4,25.3,22.5,22.0,18.6,14.0,14.0$. 
DART-TOF LC/MS m/z calcd. for $\mathrm{C}_{20} \mathrm{H}_{31} \mathrm{O}_{4}\left[\mathrm{M}+\mathrm{H}^{+}\right] 335.2222$, found 335.2226.

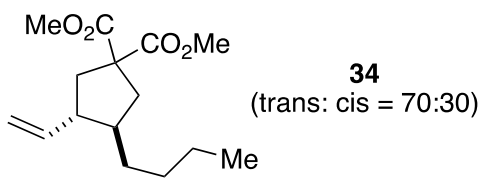

Prepared according to Procedure $\boldsymbol{C}$. Colorless oil (31.2 $\mathrm{mg}, 58 \%$ yield) as a mixture of diastereomers (trans : $\operatorname{cis}=70: 30) . \mathrm{R}_{\mathrm{f}}=0.45(10 \%$ EtOAc in hexane).

${ }^{1} \mathbf{H}$ NMR $\left(400 \mathrm{MHz}, \mathrm{CDCl}_{3}\right) \delta 5.80-5.50(\mathrm{~m}, 1 \mathrm{H}), 5.06-4.91(\mathrm{~m}, 2.0 \mathrm{H}), 3.76-3.66(\mathrm{~m}$, $6.0 \mathrm{H}), 2.76-2.62(\mathrm{~m}, 0.70 \mathrm{H}), 2.59-2.51(\mathrm{~m}, 0.30 \mathrm{H}), 2.47(\mathrm{dd}, J=13.9,7.1 \mathrm{~Hz}, 1.0 \mathrm{H})$, $2.40-2.31(\mathrm{~m}, 0.70 \mathrm{H}), 2.23-1.89(\mathrm{~m}, 3.0 \mathrm{H}), 1.78(\mathrm{dd}, J=13.4,10.8 \mathrm{~Hz}, 0.30 \mathrm{H}), 1.67-$ $1.05(\mathrm{~m}, 6.0 \mathrm{H}), 0.90-0.82(\mathrm{~m}, 3.0 \mathrm{H})$.

${ }^{13} \mathrm{C}$ NMR $\left(101 \mathrm{MHz}, \mathrm{CDCl}_{3}\right) \delta$ 168.6, 168.5, 168.4, 168.4, 135.6, 133.6, 110.5, 110.4, 72.6, 72.3, 72.0, 54.2, 53.6, 48.0, 48.0, 45.7, 41.6, 40.5, 38.3, 35.9, 35.6, 34.5, 28.1, 25.6, 25.6, 25.3, 18.1, 18.1, 9.3, 9.2.

DART-TOF LC/MS m/z calcd. for $\mathrm{C}_{15} \mathrm{H}_{25} \mathrm{O}_{4}\left[\mathrm{M}+\mathrm{H}^{+}\right] 269.1753$, found 269.1757.

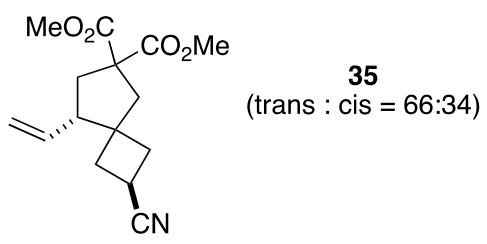

Prepared according to Procedure $\boldsymbol{C}$. Colorless oil (39.8 $\mathrm{mg}, 72 \%$ yield) as a mixture of diastereomers (trans : $\operatorname{cis}=66: 34) . \mathrm{R}_{\mathrm{f}}=0.30(20 \%$ EtOAc in hexane).

${ }^{1} \mathbf{H}$ NMR $\left(400 \mathrm{MHz}, \mathrm{CDCl}_{3}\right) \delta 5.84-5.64(\mathrm{~m}, 1.0 \mathrm{H}), 5.31-4.96(\mathrm{~m}, 2.0 \mathrm{H}), 3.90-3.45$ $(\mathrm{m}, 6.0 \mathrm{H}), 3.07-2.92(\mathrm{~m}, 0.34 \mathrm{H}), 2.88-2.73(\mathrm{~m}, 0.66 \mathrm{H}), 2.61(\mathrm{dd}, J=27.5,14.0 \mathrm{~Hz}$, $1.0 \mathrm{H}), 2.52-1.88(\mathrm{~m}, 8.0 \mathrm{H})$.

${ }^{13} \mathrm{C}$ NMR $\left(101 \mathrm{MHz}, \mathrm{CDCl}_{3}\right) \delta 172.5,172.5,172.5,136.0,135.7,122.4,122.0,118.4$, $118.3,57.4,57.3,52.3,52.9,51.5,51.4,47.5$, 47.3, 47.2, 45.3, 37.9, 37.7, 34.4, 34.4, 33.3, 32.4, 16.9, 16.4 .

DART-TOF LC/MS m/z calcd. for $\mathrm{C}_{15} \mathrm{H}_{23} \mathrm{~N}_{2} \mathrm{O}_{4}\left[\mathrm{M}+\mathrm{NH}_{4}{ }^{+}\right]$295.1658, found 295.1656.

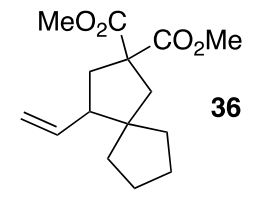

Prepared according to Procedure $\boldsymbol{C}(0.4 \mathrm{mmol})$. Colorless oil $(67.2 \mathrm{mg}, 63 \%$ yield $) . \mathrm{R}_{\mathrm{f}}=$ 0.45 (10\% EtOAc in hexane).

${ }^{1} \mathbf{H}$ NMR $\left(400 \mathrm{MHz}, \mathrm{CDCl}_{3}\right) \delta 5.78-5.60(\mathrm{~m}, 1 \mathrm{H}), 5.08-4.91(\mathrm{~m}, 2 \mathrm{H}), 3.70(\mathrm{~s}, 3 \mathrm{H})$, $3.69(\mathrm{~s}, 3 \mathrm{H}), 2.57-2.31(\mathrm{~m}, 3 \mathrm{H}), 2.19-2.01(\mathrm{~m}, 2 \mathrm{H}), 1.65-1.13(\mathrm{~m}, 8 \mathrm{H})$.

${ }^{13} \mathrm{C}$ NMR $\left(101 \mathrm{MHz}, \mathrm{CDCl}_{3}\right) \delta 173.4,173.2,137.6,116.2,57.8,53.7,52.7,52.7,51.8$, 47.4, 39.2, 37.2, 32.1, 24.7, 24.4.

DART-TOF LC/MS m/z calcd. for $\mathrm{C}_{15} \mathrm{H}_{23} \mathrm{O}_{4}\left[\mathrm{M}+\mathrm{H}^{+}\right] 267.1596$, found 267.1600. 


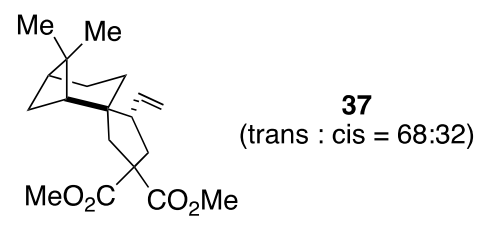

Prepared according to Procedure $\boldsymbol{C}$. Colorless oil (43.5 $\mathrm{mg}, 68 \%$ yield) as a mixture of diastereomers (trans : $\operatorname{cis}=68: 32) . \mathrm{R}_{\mathrm{f}}=0.40(10 \%$ EtOAc in hexane).

${ }^{1} \mathbf{H}$ NMR $\left(400 \mathrm{MHz}, \mathrm{CDCl}_{3}\right) \delta 5.97-5.82(\mathrm{~m}, 0.68 \mathrm{H}), 5.72-5.52(\mathrm{~m}, 0.32 \mathrm{H}), 5.09-$ $4.81(\mathrm{~m}, 2.0 \mathrm{H}), 3.75-3.65(\mathrm{~m}, 6.0 \mathrm{H}), 2.60(\mathrm{~d}, J=14.1 \mathrm{~Hz}, 0.68 \mathrm{H}), 2.50-2.24(\mathrm{~m}$, $2.64 \mathrm{H}), 2.18(\mathrm{~d}, J=14.1 \mathrm{~Hz}, 0.68 \mathrm{H}), 2.12-1.97(\mathrm{~m}, 2.0 \mathrm{H}), 1.94-1.59(\mathrm{~m}, 5.32 \mathrm{H}), 1.45$ $-1.22(\mathrm{~m}, 1.68 \mathrm{H}), 1.17(\mathrm{~s}, 3.0 \mathrm{H}), 0.99(\mathrm{~s}, 2.04 \mathrm{H}), 0.97(\mathrm{~s}, 0.96 \mathrm{H})$.

${ }^{13} \mathrm{C}$ NMR $\left(101 \mathrm{MHz}, \mathrm{CDCl}_{3}\right) \delta 173.5,173.4,173.3,173.3,139.1,138.9,115.9,114.8$, 59.4, 57.8, 55.9, 53.5, 52.8, 52.7, 52.7, 50.6, 50.2, 49.3, 48.4, 47.2, 40.2, 39.8, 39.2, 38.9, 38.3, 37.8, 29.6, 28.1, 27.8, 27.8, 27.6, 25.6, 25.4, 23.9, 23.8, 22.6.

DART-TOF LC/MS m/z calcd. for $\mathrm{C}_{19} \mathrm{H}_{32} \mathrm{NO}_{4}\left[\mathrm{M}+\mathrm{NH}_{4}{ }^{+}\right] 338.2331$, found 338.2326.

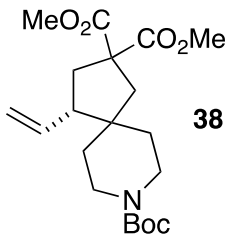

Prepared according to Procedure $\boldsymbol{C}$. Colorless oil (50.3 mg, $66 \%$ yield). $\mathrm{R}_{\mathrm{f}}=0.30(30 \%$ EtOAc in hexane).

${ }^{1}$ H NMR $\left(400 \mathrm{MHz}, \mathrm{CDCl}_{3}\right) \delta 5.63(\mathrm{ddd}, J=17.0,10.3,7.7 \mathrm{~Hz}, 1 \mathrm{H}), 5.11-4.92(\mathrm{~m}, 2 \mathrm{H})$, 3.89 (brs, 2H), $3.70(\mathrm{~s}, 3 \mathrm{H}), 3.70(\mathrm{~s}, 3 \mathrm{H}), 2.93-2.67(\mathrm{~m}, 2 \mathrm{H}), 2.57(\mathrm{~d}, J=14.2 \mathrm{~Hz}, 1 \mathrm{H})$, $2.48(\mathrm{dd}, J=12.9,6.0 \mathrm{~Hz}, 1 \mathrm{H}), 2.35-2.11(\mathrm{~m}, 2 \mathrm{H}), 2.01(\mathrm{dd}, J=14.1,1.5 \mathrm{~Hz}, 1 \mathrm{H}), 1.64$ $-1.50(\mathrm{~m}, 1 \mathrm{H}), 1.41$ (s, 9H), $1.34-1.13(\mathrm{~m}, 3 \mathrm{H})$.

${ }^{13} \mathrm{C}$ NMR $\left(101 \mathrm{MHz}, \mathrm{CDCl}_{3}\right) \delta 173.1,173.0,154.8,136.3,117.1,79.3,57.8,53.6,52.9$, 52.8, 44.3, 41.7, 37.5, 36.1, 29.7, 28.4.

DART-TOF LC/MS m/z calcd. for $\mathrm{C}_{20} \mathrm{H}_{32} \mathrm{NO}_{6}\left[\mathrm{M}+\mathrm{H}^{+}\right]$382.2230, found 382.2232.

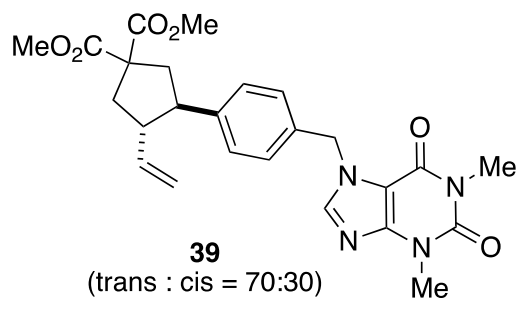

Prepared according to Procedure $\boldsymbol{C}$. Colorless oil (82.5 $\mathrm{mg}, 86 \%$ yield) as a mixture of diastereomers (trans : cis $=70: 30) . R_{f}=0.30(30 \%$ acetone in hexane).

${ }^{1} \mathbf{H}$ NMR $\left(400 \mathrm{MHz}, \mathrm{CDCl}_{3}\right) \delta 7.52(\mathrm{~s}, 0.30 \mathrm{H}), 7.51(\mathrm{~s}, 0.70 \mathrm{H}), 7.23-7.04(\mathrm{~m}, 4.0 \mathrm{H})$, 5.54 (ddd, $J=17.2,10.5,7.0 \mathrm{~Hz}, 0.30 \mathrm{H}), 5.40(\mathrm{~s}, 2.0 \mathrm{H}), 5.25$ (ddd, $J=17.1,10.3,8.0 \mathrm{~Hz}$, $0.70 \mathrm{H}), 4.86-4.68(\mathrm{~m}, 2.0 \mathrm{H}), 3.90-3.58(\mathrm{~m}, 6.0 \mathrm{H}), 3.49(\mathrm{~s}, 3 \mathrm{H}), 3.42-3.26(\mathrm{~m}$, 
$3.70 \mathrm{H}), 3.00-2.88(\mathrm{~m}, 0.70 \mathrm{H}), 2.86-2.74(\mathrm{~m}, 0.30 \mathrm{H}), 2.72-2.41(\mathrm{~m}, 3.0 \mathrm{H}), 2.33-$ $2.16(\mathrm{~m}, 1.0 \mathrm{H}), 2.14-2.03(\mathrm{~m}, 0.30 \mathrm{H})$.

${ }^{13}$ C NMR $\left(101 \mathrm{MHz}, \mathrm{CDCl}_{3}\right) \delta 172.9,172.8,172.7,172.5,155.2,151.6,148.8,142.1$, $141.4,140.9,138.5,137.8,133.7,133.3,129.0,128.3,128.1,127.8,115.8,115.6,106.9$, $106.9,58.8,58.0,52.9,52.8,52.8,50.9,50.7,49.9,49.9,47.7,47.3,42.2,40.2,38.9,38.0$, 29.7, 27.9.

DART-TOF LC/MS m/z calcd. for $\mathrm{C}_{25} \mathrm{H}_{29} \mathrm{~N}_{4} \mathrm{O}_{6}\left[\mathrm{M}+\mathrm{H}^{+}\right] 481.2087$, found 481.2089.

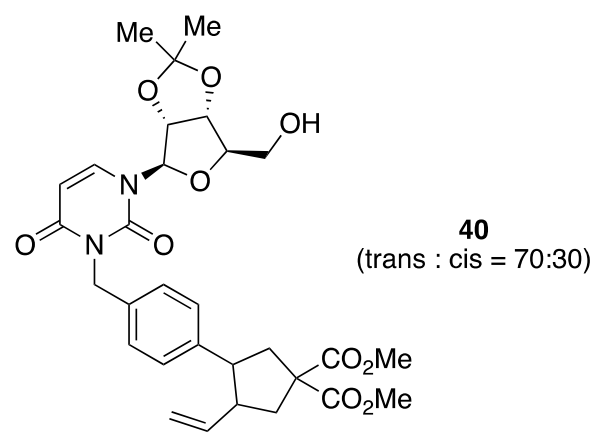

Prepared according to Procedure $\boldsymbol{C}$. Colorless oil ( $85.2 \mathrm{mg}, 73 \%$ yield) as a mixture of diastereomers (trans : cis $=70: 30)$. $\mathrm{R}_{\mathrm{f}}=0.30(30 \%$ acetone in hexane).

${ }^{1} \mathbf{H}$ NMR $\left(400 \mathrm{MHz}, \mathrm{CDCl}_{3}\right) \delta 7.40-7.31(\mathrm{~m}, 3.0 \mathrm{H}), 7.17-7.00(\mathrm{~m}, 2.0 \mathrm{H}), 5.73(\mathrm{dd}, J=$ $8.0,1.1 \mathrm{~Hz}, 1.0 \mathrm{H}), 5.66-5.54(\mathrm{~m}, 1.30 \mathrm{H}), 5.33(\mathrm{ddd}, J=17.1,10.3,7.9 \mathrm{~Hz}, 0.70 \mathrm{H}), 5.12$ $-4.76(\mathrm{~m}, 6.0 \mathrm{H}), 4.28(\mathrm{q}, J=2.9 \mathrm{~Hz}, 1.0 \mathrm{H}), 3.94-3.82(\mathrm{~m}, 1.0 \mathrm{H}), 3.81-3.69(\mathrm{~m}, 7.0 \mathrm{H})$, $3.43-3.30(\mathrm{~m}, 0.70 \mathrm{H}), 3.10-2.92(\mathrm{~m}, 1.70 \mathrm{H}), 2.88-2.77(\mathrm{~m}, 0.30 \mathrm{H}), 2.76-2.46(\mathrm{~m}$, $3.0 \mathrm{H}), 2.36(\mathrm{dd}, J=14.0,6.0 \mathrm{~Hz}, 0.70 \mathrm{H}), 2.32-2.22(\mathrm{~m}, 0.30 \mathrm{H}), 2.19-2.07(\mathrm{~m}, 0.30 \mathrm{H})$, $1.55(\mathrm{~s}, 3.0 \mathrm{H}), 1.34(\mathrm{~s}, 3.0 \mathrm{H})$.

${ }^{13} \mathrm{C}$ NMR $\left(101 \mathrm{MHz}, \mathrm{CDCl}_{3}\right) \delta 173.1,172.9,172.9,172.6,162.6,151.0,141.1,140.5$, 140.3, 138.6, 138.1, 134.6, 134.3, 129.3, 128.9, 128.4, 127.6, 115.7, 115.5, 114.2, 102.0, $96.4,96.4,86.9,84.0,80.4,62.6,58.9,58.1,52.9,52.9,52.8,51.0,50.6,50.5,47.7,47.2$, $43.9,42.5,40.2,38.9,38.1,27.3,25.3$.

ESI-TOF LC/MS m/z calcd. for $\mathrm{C}_{30} \mathrm{H}_{37} \mathrm{~N}_{2} \mathrm{O}_{10}\left[\mathrm{M}+\mathrm{H}^{+}\right]$585.2448, found 585.2453.

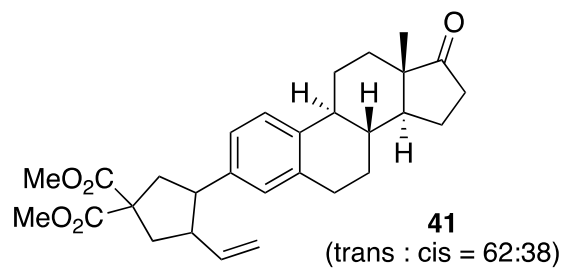

Prepared according to Procedure $\boldsymbol{C}$. Colorless oil (50.8 mg, 55\% yield) as a mixture of diastereomers (trans : $c$ is $=62: 38) . \mathrm{R}_{\mathrm{f}}=0.35(20 \%$ EtOAc in hexane).

${ }^{1} \mathbf{H}$ NMR $\left(400 \mathrm{MHz}, \mathrm{CDCl}_{3}\right) \delta 7.24-7.15(\mathrm{~m}, 1.0 \mathrm{H}), 7.05-6.82(\mathrm{~m}, 2.0 \mathrm{H}), 5.73-5.58$ $(\mathrm{m}, 0.38 \mathrm{H}), 5.49-5.32(\mathrm{~m}, 0.62 \mathrm{H}), 5.01-4.76(\mathrm{~m}, 2.0 \mathrm{H}), 4.00-3.55(\mathrm{~m}, 6.0 \mathrm{H}), 3.44-$ $3.27(\mathrm{~m}, 0.62 \mathrm{H}), 3.06-2.95(\mathrm{~m}, 0.62 \mathrm{H}), 2.92-1.91(\mathrm{~m}, 13.76 \mathrm{H}), 1.74-1.37(\mathrm{~m}, 6.0 \mathrm{H})$, $0.90(\mathrm{~s}, 3.0 \mathrm{H})$.

${ }^{13}$ C NMR $\left(101 \mathrm{MHz}, \mathrm{CDCl}_{3}\right) \delta 173.1,173.0,173.0,172.9,172.7,138.9,138.9,138.4$, 138.1, 138.1, 138.0, 138.0, 137.6, 136.4, 136.4, 136.0, 129.2, 128.8, 128.4, 128.0, 126.0, 
125.7, 125.4, 125.4, 125.1, 125.0, 124.8, 115.5, 115.3, 58.9, 58.0, 52.9, 52.8, 50.9, 50.9, $50.5,50.3,50.3,48.0,47.5,47.5,47.1,47.1,44.3,44.3,42.7,42.6,40.2,39.0,38.3,38.2$, 38.2, 38.1, 35.9, 31.6, 29.7, 29.5, 29.4, 26.6, 25.7, 21.6, 13.9 .

DART-TOF LC/MS m/z calcd. for $\mathrm{C}_{29} \mathrm{H}_{37} \mathrm{O}_{5}\left[\mathrm{M}+\mathrm{H}^{+}\right]$465.2641, found 465.2642.

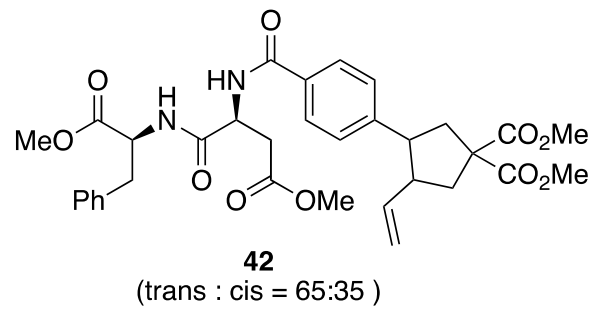

Prepared according to Procedure $\boldsymbol{C}$. Colorless oil (100.4 mg, $81 \%$ yield) as a mixture of diastereomers (trans : $\operatorname{cis}=65: 35)$. $\mathrm{R}_{\mathrm{f}}=0.30(30 \%$ acetone in hexane).

${ }^{1} \mathbf{H}$ NMR $\left(400 \mathrm{MHz}, \mathrm{CDCl}_{3}\right) \delta 7.72-7.54(\mathrm{~m}, 3.0 \mathrm{H}), 7.35-6.98(\mathrm{~m}, 8.0 \mathrm{H}), 5.69-5.56$ (m, $0.35 \mathrm{H}), 5.34$ (ddd, $J=17.1,10.3,8.2 \mathrm{~Hz}, 0.65 \mathrm{H}), 5.07-4.70(\mathrm{~m}, 4.0 \mathrm{H}), 3.83-3.76$ $(\mathrm{m}, 6.0 \mathrm{H}), 3.71(\mathrm{~s}, 6.0 \mathrm{H}), 3.55-3.44(\mathrm{~m}, 0.65 \mathrm{H}), 3.19-2.53(\mathrm{~m}, 8.0 \mathrm{H}), 2.44-2.32(\mathrm{~m}$, $1.0 \mathrm{H}), 2.26-2.13(\mathrm{~m}, 0.35 \mathrm{H})$.

${ }^{13} \mathrm{C}$ NMR $\left(101 \mathrm{MHz}, \mathrm{CDCl}_{3}\right) \delta 173.1,173.1,173.0,172.9,172.8,172.5,171.4,170.2$, 167.0, 146.2, 145.4, 138.3, 137.7, 135.6, 135.6, 131.5, 131.1, 129.1, 128.6, 128.5, 128.5, $127.9,127.4,127.0,127.0,116.1,115.9,59.0,58.2,53.5,53.0,53.0,52.9,52.9,52.4$, 52.2, 51.3, 49.3, 47.9, 47.5, 42.1, 40.4, 39.0, 38.0, 37.9, 37.7, 34.8.

ESI-TOF LC/MS m/z calcd. for $\mathrm{C}_{33} \mathrm{H}_{42} \mathrm{~N}_{3} \mathrm{O}_{10}\left[\mathrm{M}+\mathrm{NH}_{4}{ }^{+}\right] 640.2870$, found 640.2872 .

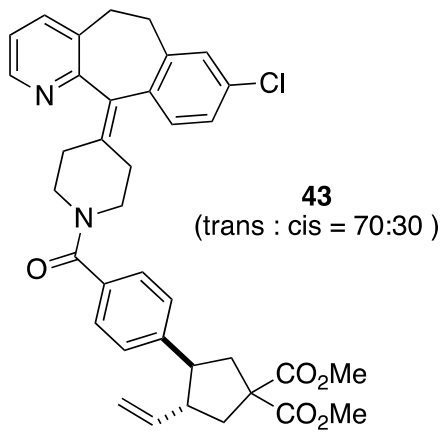

Prepared according to Procedure $\boldsymbol{C}$. Colorless oil ( $92.7 \mathrm{mg}, 74 \%$ yield) as a mixture of diastereomers (trans : cis $=70: 30)$. $R_{\mathrm{f}}=0.35$ ( $30 \%$ acetone in hexane).

${ }^{1} \mathbf{H}$ NMR $\left(400 \mathrm{MHz}, \mathrm{CDCl}_{3}\right) \delta 8.37$ (brs, $\left.1.0 \mathrm{H}\right), 7.50-6.95$ (m, 9.0H), 5.59 (ddd, $J=$ $17.2,10.5,6.9 \mathrm{~Hz}, 0.30 \mathrm{H}), 5.29(\mathrm{ddd}, J=17.0,10.3,8.0 \mathrm{~Hz}, 0.70 \mathrm{H}), 4.89-4.74(\mathrm{~m}$, $2.0 \mathrm{H}), 4.17$ (brs, $1.0 \mathrm{H}), 3.86-3.70(\mathrm{~m}, 6.0 \mathrm{H}), 3.68-3.54(\mathrm{~m}, 1 \mathrm{H}), 3.50-3.13(\mathrm{~m}$, $4.30 \mathrm{H}), 3.05-2.94(\mathrm{~m}, 0.70 \mathrm{H}), 2.91-2.08(\mathrm{~m}, 11.0 \mathrm{H})$.

${ }^{13}$ C NMR $\left(101 \mathrm{MHz}, \mathrm{CDCl}_{3}\right) \delta$ 173.0, 172.9, 172.8, 172.5, 170.4, 170.4, 146.7, 143.4, 142.7, 139.6, 138.3, 137.8, 137.6, 136.9, 134.7, 134.3, 133.9, 133.4, 133.0, 130.5, 129.0, $128.4,127.7,127.1,126.8,126.2,122.3,115.9,115.8,58.9,58.1,53.0,52.9,52.9,52.9$, 51.2, 50.9, 47.9, 47.3, 42.3, 40.3, 38.9, 38.1, 31.7, 31.6, 31.5.

ESI-TOF LC/MS m/z calcd. for $\mathrm{C}_{37} \mathrm{H}_{38} \mathrm{ClN}_{2} \mathrm{O}_{5}[\mathrm{M}+\mathrm{H}]^{+} 625.2469$, found 625.2473 . 


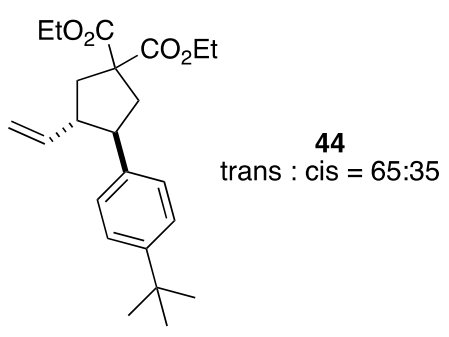

Prepared according to Procedure $\boldsymbol{C}$. Colorless oil (69.1 mg, 93\% yield) as a mixture of diastereomers (trans : $\operatorname{cis}=65: 35) . \mathrm{R}_{\mathrm{f}}=0.45(10 \%$ EtOAc in hexane).

${ }^{1} \mathbf{H}$ NMR $\left(400 \mathrm{MHz}, \mathrm{CDCl}_{3}\right) \delta 7.34-7.25(\mathrm{~m}, 2.0 \mathrm{H}), 7.20-7.02$ (m, 2.0H), 5.68 (ddd, $J$ $=17.3,10.5,6.9 \mathrm{~Hz}, 0.35 \mathrm{H}), 5.40(\mathrm{ddd}, J=17.0,10.3,7.8 \mathrm{~Hz}, 0.65 \mathrm{H}), 4.98-4.78(\mathrm{~m}$, $2.0 \mathrm{H}), 4.44-4.00(\mathrm{~m}, 4.0 \mathrm{H}), 3.45-3.33(\mathrm{~m}, 0.65 \mathrm{H}), 3.07-2.95(\mathrm{~m}, 0.65 \mathrm{H}), 2.91-2.51$ $(\mathrm{m}, 3.35 \mathrm{H}), 2.44-2.25(\mathrm{~m}, 1.0 \mathrm{H}), 2.15(\mathrm{dd}, J=13.2,10.5 \mathrm{~Hz}, 0.35 \mathrm{H}), 1.39-1.16(\mathrm{~m}$, $15.0 \mathrm{H})$.

${ }^{13} \mathbf{C}$ NMR $\left(101 \mathrm{MHz}, \mathrm{CDCl}_{3}\right) \delta 172.8,172.6,172.6,172.3,149.2,148.9,139.1,138.5$, $138.5,137.8,128.0,127.2,125.2,124.9,115.4,115.2,61.6,61.5,61.5,59.1,58.2,50.9$, 50.4, 47.6, 47.3, 42.5, 40.1, 38.8, 38.1, 34.4, 34.4, 31.4, 14.1, 14.0.

DART-TOF LC/MS m/z calcd. for $\mathrm{C}_{23} \mathrm{H}_{33} \mathrm{O}_{4}\left[\mathrm{M}+\mathrm{H}^{+}\right] 373.2379$, found 373.2381.

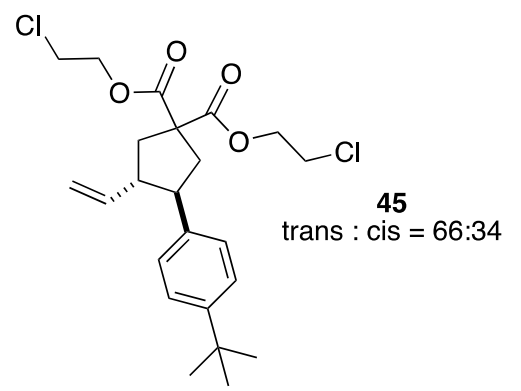

Prepared according to Procedure $\boldsymbol{C}$. Colorless oil (72.5 $\mathrm{mg}, 82 \%$ yield) as a mixture of diastereomers (trans : $\operatorname{cis}=66: 34) . \mathrm{R}_{\mathrm{f}}=0.45(10 \%$ EtOAc in hexane).

${ }^{1} \mathbf{H}$ NMR (400 MHz, $\left.\mathrm{CDCl}_{3}\right) \delta 7.34-7.28$ (m, 2.0H), $7.19-7.05$ (m, 2.0H), 5.68 (ddd, $J$ $=16.6,11.0,6.9 \mathrm{~Hz}, 0.34 \mathrm{H}), 5.41(\mathrm{ddd}, J=17.1,10.3,7.8 \mathrm{~Hz}, 0.66 \mathrm{H}), 5.01-4.78(\mathrm{~m}$, $2.0 \mathrm{H}), 4.68-4.20(\mathrm{~m}, 4.0 \mathrm{H}), 3.78-3.66(\mathrm{~m}, 4.0 \mathrm{H}), 3.45(\mathrm{dt}, J=10.5,7.4 \mathrm{~Hz}, 0.66 \mathrm{H})$, $3.11-2.98(\mathrm{~m}, 0.66 \mathrm{H}), 2.94-2.58(\mathrm{~m}, 3.34 \mathrm{H}), 2.51-2.32(\mathrm{~m}, 1.0 \mathrm{H}), 2.26-2.17(\mathrm{~m}$, $0.34 \mathrm{H}), 1.31(\mathrm{~s}, 9.0 \mathrm{H})$.

${ }^{13}$ C NMR (101 MHz, $\left.\mathrm{CDCl}_{3}\right) \delta 172.1,171.9,171.9,171.7,149.4,149.2,138.8,138.2$, $138.1,137.4,128.0,127.2$, 125.3, 125.0, 115.6, 115.5, 65.1, 65.1, 65.0, 59.0, 58.1, 50.9, 50.4, 47.6, 47.3, 42.5, 41.4, 41.4, 40.1, 38.9, 38.2, 34.4, 34.4, 31.4.

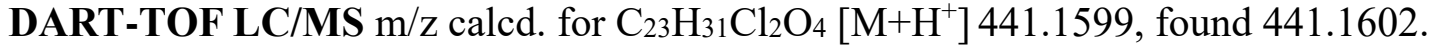




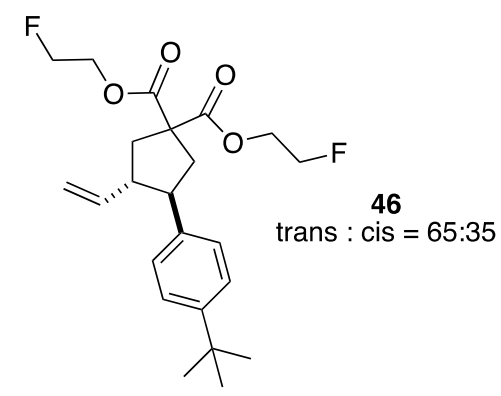

Prepared according to Procedure $\boldsymbol{C}$. Colorless oil (74.5 mg, 91\% yield) as a mixture of diastereomers (trans : $\operatorname{cis}=65: 35) . \mathrm{R}_{\mathrm{f}}=0.33(30 \%$ EtOAc in hexane).

${ }^{1} \mathbf{H}$ NMR $\left(400 \mathrm{MHz}, \mathrm{CDCl}_{3}\right) \delta 7.37-7.28(\mathrm{~m}, 2.0 \mathrm{H}), 7.20-7.04(\mathrm{~m}, 2.0 \mathrm{H}), 5.78-5.61$ $(\mathrm{m}, 0.35 \mathrm{H}), 5.41(\mathrm{ddd}, J=17.1,10.3,7.8 \mathrm{~Hz}, 0.65 \mathrm{H}), 4.99-4.81(\mathrm{~m}, 2.0 \mathrm{H}), 4.70-4.62$ $(\mathrm{m}, 2.0 \mathrm{H}), 4.58-4.52(\mathrm{~m}, 2.0 \mathrm{H}), 4.46-4.43(\mathrm{~m}, 2.0 \mathrm{H}), 4.42-4.34(\mathrm{~m}, 2.0 \mathrm{H}), 3.45(\mathrm{dt}, J$ $=10.6,7.4 \mathrm{~Hz}, 0.65 \mathrm{H}), 3.11-2.98(\mathrm{~m}, 0.65 \mathrm{H}), 2.95-2.57(\mathrm{~m}, 3.35 \mathrm{H}), 2.51-2.32(\mathrm{~m}$, $1.0 \mathrm{H}), 2.27-2.15(\mathrm{~m}, 0.35 \mathrm{H}), 1.31(\mathrm{~s}, 9.0 \mathrm{H})$.

${ }^{13} \mathrm{C}$ NMR $\left(101 \mathrm{MHz}, \mathrm{CDCl}_{3}\right) \delta 172.4,172.2,172.1,171.9,149.4,149.1,138.8,138.2$, $138.1,137.4,128.0,127.2,125.3,125.0,115.6,115.4,81.8,80.2,80.1,64.6,64.5,64.5$, 64.4, 64.3, 64.3, 58.9, 58.1, 50.8, 50.4, 47.6, 47.3, 42.5, 40.1, 38.8, 38.1, 34.4, 34.4, 31.4.

${ }^{19}$ F NMR $\left(376 \mathrm{MHz}, \mathrm{CDCl}_{3}\right) \delta$-225.09, -225.10, -225.12, -225.12, -225.16, -225.16.

DART-TOF LC/MS m/z calcd. for $\mathrm{C}_{23} \mathrm{H}_{34} \mathrm{~F}_{2} \mathrm{NO}_{4}\left[\mathrm{M}+\mathrm{NH}_{4}{ }^{+}\right] 426.2456$, found 426.2452 .

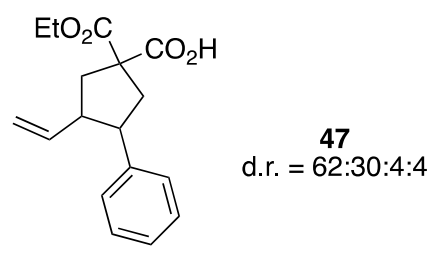

Prepared according to Procedure $\boldsymbol{C}$. Colorless oil (53.0 $\mathrm{mg}, 92 \%$ yield) as a mixture of diastereomers (d.r. $=62: 30: 4: 4) . \mathrm{R}_{\mathrm{f}}=0.35(10 \% \mathrm{MeOH}$ in $\mathrm{DCM})$.

${ }^{1} \mathbf{H}$ NMR $\left(400 \mathrm{MHz}, \mathrm{CDCl}_{3}\right) \delta 11.14$ (brs, $\left.1.0 \mathrm{H}\right), 7.43-6.79(\mathrm{~m}, 5.0 \mathrm{H}), 5.73-5.57(\mathrm{~m}$, $0.30 \mathrm{H}), 5.46-5.32(\mathrm{~m}, 0.66 \mathrm{H}), 5.28-5.22(\mathrm{~m}, 0.04 \mathrm{H}), 4.97-4.76(\mathrm{~m}, 2.0 \mathrm{H}), 4.37-$ $4.12(\mathrm{~m}, 2.0 \mathrm{H}), 3.58-3.40(\mathrm{~m}, 0.66 \mathrm{H}), 3.14-2.98(\mathrm{~m}, 0.66 \mathrm{H}), 2.97-1.93(\mathrm{~m}, 4.68 \mathrm{H})$, $1.45-1.20(\mathrm{~m}, 3.0 \mathrm{H})$.

${ }^{13}$ C NMR (101 MHz, $\left.\mathrm{CDCl}_{3}\right) \delta 178.6,178.3,178.1,172.1,171.8,141.3,140.7,140.6$, 138.6, 138.1, 138.0, 128.4, 128.3, 128.1, 127.6, 126.7, 126.3, 115.8, 115.8, 115.5, 115.5, 62.1, 62.0, 59.1, 59.1, 58.1, 51.6, 50.9, 50.9, 48.1, 48.0, 47.4, 42.6, 42.5, 40.4, 40.3, 39.0, 39.0, 38.1, 38.1, 14.0 .

DART-TOF LC/MS m/z calcd. for $\mathrm{C}_{17} \mathrm{H}_{21} \mathrm{O}_{4}\left[\mathrm{M}+\mathrm{H}^{+}\right] 289.1440$, found 289.1441.

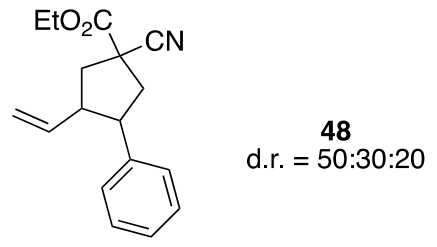


Prepared according to Procedure $\boldsymbol{C}$. Colorless oil (45.1 mg, $84 \%$ yield) as a mixture of diastereomers (d.r. $=50: 30: 20) . \mathrm{R}_{\mathrm{f}}=0.35(30 \%$ EtOAc in hexane).

${ }^{1} \mathbf{H}$ NMR $\left(400 \mathrm{MHz}, \mathrm{CDCl}_{3}\right) \delta 7.32-6.99$ (m, 5.0H), $5.67-5.50$ (m, 0.30H), $5.42-5.31$ (m, 0.20H), 5.25 (ddd, $J=17.1,10.2,8.1 \mathrm{~Hz}, 0.50 \mathrm{H}), 4.99-4.68(\mathrm{~m}, 2.0 \mathrm{H}), 4.40-4.10$ $(\mathrm{m}, 2.0 \mathrm{H}), 3.68-3.56(\mathrm{~m}, 0.50 \mathrm{H}), 3.52-3.41(\mathrm{~m}, 0.20 \mathrm{H}), 3.20-3.12(\mathrm{~m}, 0.50 \mathrm{H}), 3.09-$ $2.31(\mathrm{~m}, 4.50 \mathrm{H}), 2.27-2.15(\mathrm{~m}, 0.30 \mathrm{H}), 1.36-1.21(\mathrm{~m}, 3.0 \mathrm{H})$.

${ }^{13} \mathrm{C}$ NMR $\left(101 \mathrm{MHz} \mathrm{CDCl}_{3}\right) \delta 169.9,169.7,169.6,169.1,140.5,139.9,139.7,139.1$, $137.7,137.2$, 137.1, 136.5, 128.7, 128.6, 128.4, 128.3, 128.3, 128.2, 127.6, 127.5, 127.1, 127.0, 126.7, 121.3, 121.2, 121.1, 116.7, 116.7, 116.6, 116.1, 63.1, 51.7, 51.6, 51.2, 50.5, 48.5, 47.8, 47.8, 47.3, 47.0, 45.2, 45.1, 43.3, 43.1, 42.0, 41.6, 41.6, 40.8, 14.0, 14.0.

DART-TOF LC/MS m/z calcd. for $\mathrm{C}_{17} \mathrm{H}_{23} \mathrm{~N}_{2} \mathrm{O}_{2}\left[\mathrm{M}+\mathrm{NH}_{4}{ }^{+}\right]$287.1760, found 287.1758.

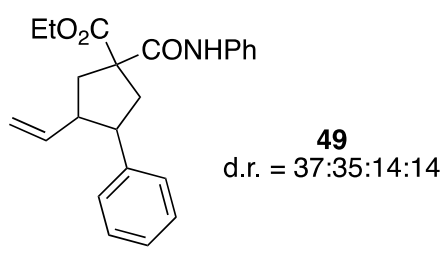

Prepared according to Procedure $\boldsymbol{C}$. Colorless oil (67.6 mg, 93\% yield) as a mixture of diastereomers (d.r. $=37: 35: 14: 14) . \mathrm{R}_{\mathrm{f}}=0.45(30 \%$ EtOAc in hexane).

${ }^{1} \mathbf{H}$ NMR $\left(400 \mathrm{MHz}, \mathrm{CDCl}_{3}\right) \delta 8.33$ (brs, 0.28H), 8.07 (brs, 0.37H), 7.99 (brs, 0.35H), $7.52-7.39(\mathrm{~m}, 2.0 \mathrm{H}), 7.32-6.96(\mathrm{~m}, 8.0 \mathrm{H}), 5.70-5.49(\mathrm{~m}, 0.30 \mathrm{H}), 5.32$ (dddd, $J=$ $17.1,10.4,7.9,1.7 \mathrm{~Hz}, 0.70 \mathrm{H}), 4.92-4.66(\mathrm{~m}, 2.0 \mathrm{H}), 4.37-4.08(\mathrm{~m}, 2.0 \mathrm{H}), 3.46$ (dt, $J=$ $10.8,7.4 \mathrm{~Hz}, 0.37 \mathrm{H}), 3.36(\mathrm{dt}, J=10.6,7.7 \mathrm{~Hz}, 0.35 \mathrm{H}), 3.09-2.40(\mathrm{~m}, 4.86 \mathrm{H}), 2.39-$ $2.29(\mathrm{~m}, 0.28 \mathrm{H}), 2.22-2.12(\mathrm{~m}, 0.14 \mathrm{H}), 1.36-1.19(\mathrm{~m}, 3.0 \mathrm{H})$.

${ }^{13} \mathrm{C}$ NMR $\left(101 \mathrm{MHz}, \mathrm{CDCl}_{3}\right) \delta 175.1,174.8,174.7,169.1,168.7,168.1,141.6,141.0$, $140.9,138.9,138.8,138.5,138.2,137.9,137.8,129.1,129.0,128.5,128.4,128.3,128.1$, $128.0,127.7,127.6,126.6,126.6,126.3,126.2$, 124.5, 124.5, 120.0, 120.0, 119.9, 119.9, 115.6, 115.6, 115.4, 115.3, 62.5, 62.4, 62.3, 61.1, 60.5, 59.3, 52.0, 51.5, 51.3, 51.0, 48.1, 48.0, 47.4, 47.3, 43.1, 42.9, 41.1, 39.5, 39.4, 38.2, 37.7, 14.1, 14.0.

DART-TOF LC/MS m/z calcd. for $\mathrm{C}_{23} \mathrm{H}_{29} \mathrm{~N}_{2} \mathrm{O}_{3}\left[\mathrm{M}+\mathrm{NH}_{4}{ }^{+}\right] 381.2178$, found 381.2173.

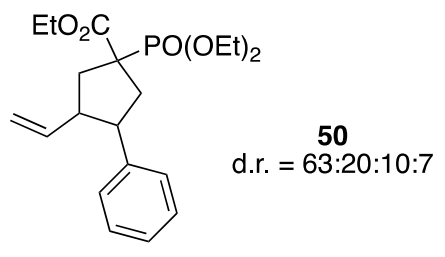

Prepared according to Procedure $\boldsymbol{C}$. Colorless oil (65.7 $\mathrm{mg}, 86 \%$ yield) as a mixture of diastereomers (d.r. $=37: 35: 14: 14) . R_{f}=0.35(30 \%$ acetone in hexane).

${ }^{1} \mathbf{H}$ NMR $\left(400 \mathrm{MHz}, \mathrm{CDCl}_{3}\right) \delta 7.26-7.05(\mathrm{~m}, 5.0 \mathrm{H}), 5.56(\mathrm{ddd}, J=17.2,9.9,7.2 \mathrm{~Hz}$, $0.32 \mathrm{H}), 5.28(\mathrm{ddd}, J=17.1,10.2,8.4 \mathrm{~Hz}, 0.68 \mathrm{H}), 4.91-4.57(\mathrm{~m}, 2.0 \mathrm{H}), 4.35-3.92(\mathrm{~m}$, $6.0 \mathrm{H}), 3.52-3.15(\mathrm{~m}, 0.73 \mathrm{H}), 3.04-2.03(\mathrm{~m}, 5.27 \mathrm{H}), 1.54-1.02(\mathrm{~m}, 9.0 \mathrm{H})$.

${ }^{13} \mathrm{C}$ NMR $\left(101 \mathrm{MHz}, \mathrm{CDCl}_{3}\right) \delta 172.3,171.8,171.7,141.9,141.4,141.3,139.2,138.9$, $138.5,137.4$, 128.6, 128.4, 128.2, 128.0, 128.0, 127.7, 127.6, 126.6, 126.5, 126.2, 126.1, 
115.8, 115.7, 115.4, 114.9, 63.1, 63.0, 62.9, 62.9, 62.9, 62.8, 62.8, 62.7, 61.8, 61.8, 55.0, 53.6, 51.6, 51.6, 51.5, 51.1, 51.0, 50.8, 50.8, 47.8, 47.7, 47.6, 47.4, 47.2, 41.1, 41.1, 40.2, 38.0, 38.0, 37.6, 37.6, 37.3, 37.3, 16.5, 16.5, 16.4, 16.4, 14.1 .

${ }^{31} \mathbf{P}$ NMR $\left(162 \mathrm{MHz}, \mathrm{CDCl}_{3}\right) \delta 27.3,26.6,26.1$.

DART-TOF LC/MS m/z calcd. for $\mathrm{C}_{20} \mathrm{H}_{33} \mathrm{NPO}_{5}\left[\mathrm{M}+\mathrm{NH}_{4}{ }^{+}\right]$398.2096, found 398.2100.

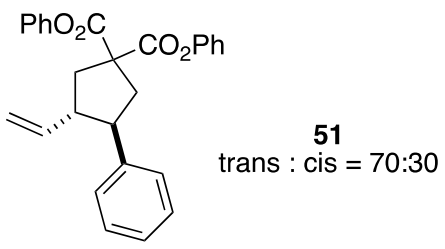

Prepared according to Procedure $\boldsymbol{C}$. Colorless oil (75.9 mg, 92\% yield) as a mixture of diastereomers (trans : $c$ is $=70: 30) . \mathrm{R}_{\mathrm{f}}=0.35(10 \%$ EtOAc in hexane).

${ }^{1} \mathbf{H}$ NMR $\left(400 \mathrm{MHz}, \mathrm{CDCl}_{3}\right) \delta 7.43-6.94(\mathrm{~m}, 15.0 \mathrm{H}), 5.64(\mathrm{ddd}, J=17.3,9.9,7.0 \mathrm{~Hz}$, $0.30 \mathrm{H}), 5.39(\mathrm{ddd}, J=17.1,10.3,7.9 \mathrm{~Hz}, 0.70 \mathrm{H}), 4.95-4.76(\mathrm{~m}, 2.0 \mathrm{H}), 3.54(\mathrm{dt}, J=$ 10.6, $7.4 \mathrm{~Hz}, 0.70 \mathrm{H}), 3.17-3.04(\mathrm{~m}, 0.70 \mathrm{H}), 3.02-2.74(\mathrm{~m}, 3.30 \mathrm{H}), 2.65-2.50(\mathrm{~m}$, $1.0 \mathrm{H}), 2.45-2.31(\mathrm{~m}, 0.30 \mathrm{H})$.

${ }^{13}$ C NMR $\left(101 \mathrm{MHz}, \mathrm{CDCl}_{3}\right) \delta 171.1,170.6,150.7,150.7,141.2,140.4,138.4,137.8$, 129.6, 128.6, 128.4, 128.2, 127.7, 126.8, 126.5, 126.3, 121.2, 121.2, 116.0, 115.9, 59.3, 58.4, 51.6, 50.9, 48.2, 47.6, 42.6, 40.4, 39.1, 38.2.

DART-TOF LC/MS m/z calcd. for $\mathrm{C}_{27} \mathrm{H}_{28} \mathrm{NO}_{4}\left[\mathrm{M}+\mathrm{NH}_{4}{ }^{+}\right] 430.2018$, found 430.2015.

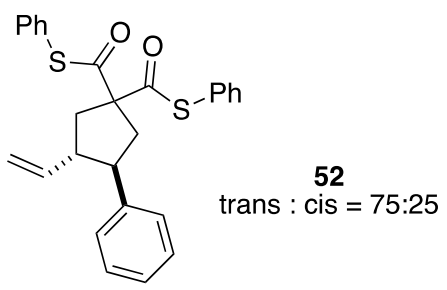

Prepared according to Procedure $\boldsymbol{C}$. Colorless oil (69.4 mg, $78 \%$ yield) as a mixture of diastereomers (trans : $c$ is $=75: 25)$. $\mathrm{R}_{\mathrm{f}}=0.40(30 \%$ EtOAc in hexane).

${ }^{1} \mathbf{H}$ NMR $\left(400 \mathrm{MHz}, \mathrm{CDCl}_{3}\right) \delta 7.53-6.96(\mathrm{~m}, 15.0 \mathrm{H}), 5.68-5.48(\mathrm{~m}, 0.25 \mathrm{H}), 5.27$ (ddd, $J=17.6,10.3,7.8 \mathrm{~Hz}, 0.75 \mathrm{H}), 4.94-4.65(\mathrm{~m}, 2.0 \mathrm{H}), 3.46-3.31(\mathrm{~m}, 0.75 \mathrm{H}), 3.07-2.41$ $(\mathrm{m}, 5.0 \mathrm{H}), 2.30(\mathrm{dd}, J=13.8,10.8 \mathrm{~Hz}, 0.25 \mathrm{H})$.

${ }^{13}$ C NMR $\left(101 \mathrm{MHz}, \mathrm{CDCl}_{3}\right) \delta 196.8,196.6,196.5,196.0,141.1,140.4,138.4,137.8$, $134.8,134.8,134.7,129.8,129.4,129.4,128.5,128.4,128.2$, 127.6, 127.0, 127.0, 126.8, 126.5, 116.1, 115.8, 74.8, 73.8, 51.3, 50.6, 47.6, 46.9, 42.3, 40.1, 38.8, 38.0.

DART-TOF LC/MS m/z calcd. for $\mathrm{C}_{27} \mathrm{H}_{28} \mathrm{NO}_{2} \mathrm{~S}_{2}\left[\mathrm{M}+\mathrm{NH}_{4}{ }^{+}\right] 462.1561$, found 462.1560 .

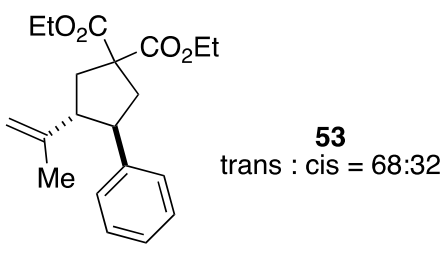


Prepared according to Procedure $\boldsymbol{C}$. Colorless oil (52.0 mg, 79\% yield) as a mixture of diastereomers (trans : $\operatorname{cis}=68: 32) . \mathrm{R}_{\mathrm{f}}=0.35(4: 1$ hex:EtOAc).

${ }^{1} \mathbf{H}$ NMR $\left(400 \mathrm{MHz}, \mathrm{CDCl}_{3}\right) \delta 7.27$ - 6.99 (m, 5.0H), $4.64-4.50$ (m, 2.0H), 4.26 - 4.09 $(\mathrm{m}, 4.0 \mathrm{H}), 3.49-3.36(\mathrm{~m}, 0.68 \mathrm{H}), 3.06-2.64(\mathrm{~m}, 2.32 \mathrm{H}), 2.56(\mathrm{dd}, J=13.6,7.4 \mathrm{~Hz}$, $0.32 \mathrm{H}), 2.49-2.34(\mathrm{~m}, 2.0 \mathrm{H}), 2.18(\mathrm{ddd}, J=25.3,13.7,11.6 \mathrm{~Hz}, 0.68 \mathrm{H}), 1.56(\mathrm{~s}, 0.96 \mathrm{H})$, $1.24-1.10(\mathrm{~m}, 8.04 \mathrm{H})$.

${ }^{13} \mathrm{C}$ NMR $\left(101 \mathrm{MHz}, \mathrm{CDCl}_{3}\right) \delta 172.7,172.6,172.6,172.1,145.0,144.1,142.4,142.1$, $128.4,127.8,127.4,126.5,126.1,112.1,111.7,61.6,61.5,59.3,57.9,53.5,49.9,49.1$, 46.8, 42.8, 40.1, 39.5, 37.8, 22.7, 19.8, 14.1.

DART-TOF LC/MS m/z calcd. for $\mathrm{C}_{20} \mathrm{H}_{30} \mathrm{NO}_{4}\left[\mathrm{M}+\mathrm{NH}_{4}{ }^{+}\right] 348.2175$, found 348.2171.

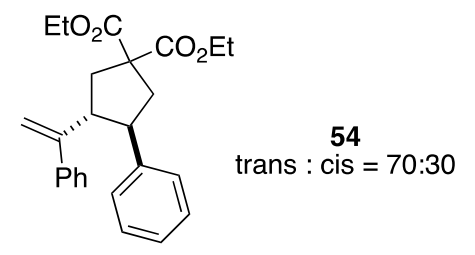

Prepared according to Procedure $\boldsymbol{C}$. Colorless oil (63.2 $\mathrm{mg}, 81 \%$ yield) as a mixture of diastereomers (trans : cis $=70: 30) . \mathrm{R}_{\mathrm{f}}=0.35(10 \%$ EtOAc in hexane).

${ }^{1} \mathbf{H}$ NMR $\left(400 \mathrm{MHz}, \mathrm{CDCl}_{3}\right) \delta 7.25-6.58(\mathrm{~m}, 10.0 \mathrm{H}), 5.10(\mathrm{~d}, J=0.9 \mathrm{~Hz}, 0.30 \mathrm{H}), 5.02$ $(\mathrm{d}, J=0.8 \mathrm{~Hz}, 0.30 \mathrm{H}), 4.95(\mathrm{~d}, J=0.9 \mathrm{~Hz}, 0.70 \mathrm{H}), 4.75(\mathrm{~d}, J=0.8 \mathrm{~Hz}, 0.70 \mathrm{H}), 4.31-$ $4.04(\mathrm{~m}, 4.0 \mathrm{H}), 3.64-3.51(\mathrm{~m}, 0.70 \mathrm{H}), 3.42(\mathrm{td}, J=8.7,5.9 \mathrm{~Hz}, 0.70 \mathrm{H}), 3.32-3.11(\mathrm{~m}$, $0.60 \mathrm{H}), 2.95(\mathrm{ddd}, J=14.5,8.6,1.2 \mathrm{~Hz}, 0.70 \mathrm{H}), 2.84(\mathrm{dd}, J=13.8,7.5 \mathrm{~Hz}, 0.30 \mathrm{H}), 2.75$ $(\mathrm{dd}, J=13.6,6.9 \mathrm{~Hz}, 0.30 \mathrm{H}), 2.64(\mathrm{dd}, J=13.2,12.1 \mathrm{~Hz}, 0.70 \mathrm{H}), 2.52$ (ddd, $J=13.2,6.1$, $1.2 \mathrm{~Hz}, 0.70 \mathrm{H}), 2.38(\mathrm{dd}, J=14.5,5.9 \mathrm{~Hz}, 0.70 \mathrm{H}), 2.21(\mathrm{dd}, J=13.8,10.9 \mathrm{~Hz}, 0.30 \mathrm{H})$, $2.01(\mathrm{dd}, J=13.6,11.2 \mathrm{~Hz}, 0.30 \mathrm{H}), 1.34-1.08(\mathrm{~m}, 6.0 \mathrm{H})$.

${ }^{13} \mathrm{C}$ NMR $\left(101 \mathrm{MHz}, \mathrm{CDCl}_{3}\right) \delta 172.6,172.6,172.3,172.1,148.9,148.0,142.7,142.0$, $142.0,141.9,128.7,128.4,128.1,127.9,127.5,127.4,127.2,127.1,126.5,126.5,126.3$, $125.9,113.2,113.2,61.7,61.6,61.6,61.5,59.2,57.7,50.3,49.7,47.0,46.3,42.8,41.7$, 40.8, 38.1, 14.1, 14.1, 14.0.

DART-TOF LC/MS m/z calcd. for $\mathrm{C}_{25} \mathrm{H}_{32} \mathrm{NO}_{4}\left[\mathrm{M}+\mathrm{NH}_{4}{ }^{+}\right] 410.2331$, found 410.2330.

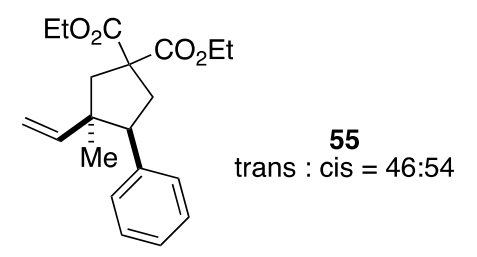

Prepared according to Procedure $\boldsymbol{C}$. Colorless oil (38.8 $\mathrm{mg}, 59 \%$ yield) as a mixture of diastereomers (trans : $\operatorname{cis}=46: 54) . \mathrm{R}_{\mathrm{f}}=0.40(10 \%$ EtOAc in hexane).

${ }^{1}$ H NMR $\left(400 \mathrm{MHz}, \mathrm{CDCl}_{3}\right) \delta 7.26-7.05(\mathrm{~m}, 5.0 \mathrm{H}), 5.83(\mathrm{dd}, J=17.4,10.7 \mathrm{~Hz}, 0.46 \mathrm{H})$, $5.46(\mathrm{dd}, J=17.4,10.9 \mathrm{~Hz}, 0.54 \mathrm{H}), 4.97-4.64(\mathrm{~m}, 2.0 \mathrm{H}), 4.26-4.05(\mathrm{~m}, 4.0 \mathrm{H}), 3.02$ $(\mathrm{dd}, J=13.4,6.6 \mathrm{~Hz}, 0.46 \mathrm{H}), 2.92(\mathrm{dd}, J=13.7,6.0 \mathrm{~Hz}, 0.54 \mathrm{H}), 2.82(\mathrm{t}, J=13.5 \mathrm{~Hz}$, $0.46 \mathrm{H}), 2.74-2.58(\mathrm{~m}, 1.08 \mathrm{H}), 2.55-2.46(\mathrm{~m}, 0.92 \mathrm{H}), 2.41-2.28(\mathrm{~m}, 1.08 \mathrm{H}), 2.22(\mathrm{~d}$, $J=14.1 \mathrm{~Hz}, 0.46 \mathrm{H}), 1.24-1.14(\mathrm{~m}, 6.0 \mathrm{H}), 1.07(\mathrm{~s}, 1.62 \mathrm{H}), 0.70(\mathrm{~s}, 1.38 \mathrm{H})$. 
${ }^{13} \mathrm{C}$ NMR $\left(101 \mathrm{MHz}, \mathrm{CDCl}_{3}\right) \delta 173.2,172.9,172.6,172.4,146.4,141.9,138.4,138.3$, $128.9,128.5,127.7,127.7,126.7,126.6,113.1,112.4,61.6,61.6,61.5,57.4,57.1,55.4$, 53.8, 48.4, 48.3, 47.0, 44.4, 38.0, 37.1, 25.7, 19.0, 14.1, 14.0.

DART-TOF LC/MS m/z calcd. for $\mathrm{C}_{20} \mathrm{H}_{30} \mathrm{NO}_{4}\left[\mathrm{M}+\mathrm{NH}_{4}{ }^{+}\right] 348.2175$, found 348.2172.

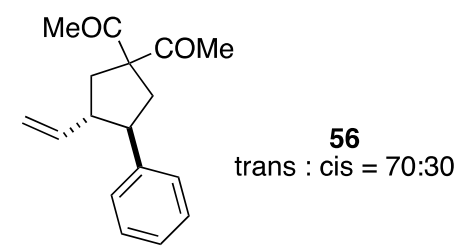

Prepared according to Procedure $\boldsymbol{C}$. Colorless oil (44.1 $\mathrm{mg}, 86 \%$ yield) as a mixture of diastereomers (trans : cis $=70: 30) . \mathrm{R}_{\mathrm{f}}=0.35$ (20\% EtOAc in hexane).

${ }^{1}$ H NMR $\left(400 \mathrm{MHz}, \mathrm{CDCl}_{3}\right) \delta 7.28-6.97(\mathrm{~m}, 5.0 \mathrm{H}), 5.61-5.44(\mathrm{~m}, 0.30 \mathrm{H}), 5.22$ (ddd, $J$ $=17.1,10.3,7.9 \mathrm{~Hz}, 0.70 \mathrm{H}), 4.90-4.66(\mathrm{~m}, 2.0 \mathrm{H}), 3.18(\mathrm{dt}, J=10.6,7.2 \mathrm{~Hz}, 0.70 \mathrm{H})$, $2.90-2.80(\mathrm{~m}, 0.70 \mathrm{H}), 2.75(\mathrm{dd}, J=13.2,7.4 \mathrm{~Hz}, 0.30 \mathrm{H}), 2.68-2.57(\mathrm{~m}, 1.40 \mathrm{H}), 2.56-$ $2.47(\mathrm{~m}, 0.30 \mathrm{H}), 2.45-2.22(\mathrm{~m}, 2.0 \mathrm{H}), 2.13-2.01(\mathrm{~m}, 6.0 \mathrm{H}), 2.00(\mathrm{dd}, J=13.3,11.2$ $\mathrm{Hz}, 0.30 \mathrm{H}), 1.84$ (dd, $J=13.3,10.8 \mathrm{~Hz}, 0.30 \mathrm{H})$.

${ }^{13}$ C NMR $\left(101 \mathrm{MHz}, \mathrm{CDCl}_{3}\right) \delta$ 204.9, 204.6, 204.5, 204.5, 141.4, 140.6, 138.7, 138.0, $128.5,128.2$, 128.1, 127.5, 126.7, 126.3, 115.7, 115.6, 74.4, 73.2, 51.2, 50.3, 47.8, 47.3, $39.3,36.9,35.5,34.3,27.0,26.4,26.3,26.0$.

DART-TOF LC/MS m/z calcd. for $\mathrm{C}_{14} \mathrm{H}_{24} \mathrm{NO}_{2}\left[\mathrm{M}+\mathrm{NH}_{4}{ }^{+}\right] 274.1807$, found 274.1804.

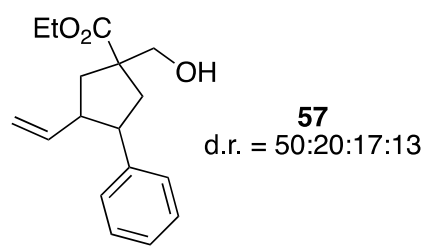

Prepared according to Procedure $\boldsymbol{C}$. Colorless oil (44.3 $\mathrm{mg}, 81 \%$ yield) as a mixture of diastereomers (d.r. $=50: 20: 17: 13) . \mathrm{R}_{\mathrm{f}}=0.30$ (30\% EtOAc in hexane).

${ }^{1}$ H NMR $\left(400 \mathrm{MHz}, \mathrm{CDCl}_{3}\right) \delta 7.33-6.96(\mathrm{~m}, 5.0 \mathrm{H}), 5.71-5.27(\mathrm{~m}, 1.0 \mathrm{H}), 4.87-4.68$ $(\mathrm{m}, 2.0 \mathrm{H}), 4.24-4.09(\mathrm{~m}, 2.0 \mathrm{H}), 3.79-3.60(\mathrm{~m}, 2.0 \mathrm{H}), 3.52(\mathrm{dt}, J=11.8,7.2 \mathrm{~Hz}$, $0.50 \mathrm{H}), 3.31(\mathrm{dt}, J=10.5,7.4 \mathrm{~Hz}, 0.17 \mathrm{H}), 3.12-1.45(\mathrm{~m}, 6.0 \mathrm{H}), 1.32-1.11(\mathrm{~m}, 3.0 \mathrm{H})$, $0.95-0.80(\mathrm{~m}, 0.33 \mathrm{H})$.

${ }^{13} \mathrm{C}$ NMR (101 MHz, $\left.\mathrm{CDCl}_{3}\right) \delta 177.7,177.6,177.6,177.4,142.0,142.0,141.3,141.3$, $139.4,139.3,139.0,138.8,128.4,128.4,128.3,128.1,128.0,127.6,127.5,126.4,126.1$, $126.1,115.3,115.2,115.2,115.0,69.2,68.1,68.0,67.5,61.2,61.1,61.1,61.1,54.3,53.8$, 52.8, 52.8, 51.8, 51.3, 51.1, 50.4, 48.2, 48.0, 47.4, 42.5, 41.6, 40.2, 39.1, 38.5, 38.2, 37.6, $36.2,14.2,14.2,14.1$.

DART-TOF LC/MS m/z calcd. for $\mathrm{C}_{17} \mathrm{H}_{26} \mathrm{NO}_{3}\left[\mathrm{M}+\mathrm{NH}_{4}{ }^{+}\right] 292.1907$, found 292.1905. 


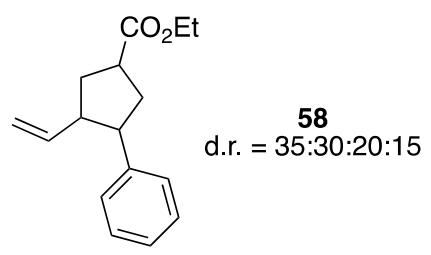

Prepared according to Procedure $\boldsymbol{C}(0.4 \mathrm{mmol})$. Colorless oil $(60.9 \mathrm{mg}, 62 \%$ yield $)$ as a mixture of diastereomers (trans $: \operatorname{cis}=35: 30: 20: 15) . \mathrm{R}_{\mathrm{f}}=0.45$ (10\% EtOAc in hexane).

${ }^{1} \mathbf{H}$ NMR (400 MHz, $\left.\mathrm{CDCl}_{3}\right) \delta 7.30-7.01$ (m, 5.0H), $5.72-5.50(\mathrm{~m}, 0.55 \mathrm{H}), 5.42-5.22$ $(\mathrm{m}, 0.45 \mathrm{H}), 4.91-4.57(\mathrm{~m}, 2.0 \mathrm{H}), 4.24-3.91(\mathrm{~m}, 2.0 \mathrm{H}), 3.45-1.74(\mathrm{~m}, 7.0 \mathrm{H}), 1.32-$ $1.04(\mathrm{~m}, 3.0 \mathrm{H})$.

${ }^{13} \mathrm{C}$ NMR $\left(101 \mathrm{MHz} \mathrm{CDCl}_{3}\right) \delta 176.9,176.4,176.3,175.9,143.2,142.6,141.8,141.5$, $140.0,139.8,139.3,138.6,128.5,128.4,128.3,128.3,128.0,128.0,127.6,127.5,126.4$, $126.3,126.1,114.9,114.8,114.6,60.5,60.5,52.6,52.3,51.2,50.8,49.0,48.8,48.1,48.1$, 42.9, 42.0, 41.8, 41.5, 38.7, 38.1, 36.6, 35.7, 34.9, 34.7, 34.2, 33.4, 14.3, 14.3.

DART-TOF LC/MS m/z calcd. for $\mathrm{C}_{14} \mathrm{H}_{24} \mathrm{NO}_{2}\left[\mathrm{M}+\mathrm{NH}_{4}{ }^{+}\right] 262.1807$, found 262.1805.

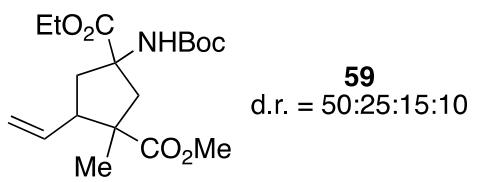

Prepared according to Procedure $\boldsymbol{C}$. Colorless oil (63.7 $\mathrm{mg}, 90 \%$ yield) as a mixture of diastereomers (trans $: \mathrm{cis}=50: 25: 15: 10) . \mathrm{R}_{\mathrm{f}}=0.35$ (20\% EtOAc in hexane).

${ }^{1}$ H NMR (400 MHz, $\left.\mathrm{CDCl}_{3}\right) \delta 5.90-5.57(\mathrm{~m}, 1.0 \mathrm{H}), 5.44-4.80(\mathrm{~m}, 3.0 \mathrm{H}), 4.28-4.04$ $(\mathrm{m}, 2.0 \mathrm{H}), 3.75-3.40(\mathrm{~m}, 3.0 \mathrm{H}), 3.36-1.72(\mathrm{~m}, 5.0 \mathrm{H}), 1.58-0.92(\mathrm{~m}, 15.0 \mathrm{H})$.

${ }^{13}$ C NMR (101 MHz, $\left.\mathrm{CDCl}_{3}\right) \delta 177.9,176.6,175.9,174.4,173.4,173.2,155.2,136.3$, 136.2 , 136.0, 135.9, 116.9, 116.7, 116.6, 65.2, 61.5, 61.4, 61.4, 61.4, 54.2, 54.0, 53.1, $52.5,52.3,52.0,51.8,51.7,51.6,51.0,49.2,48.8,48.0,42.1,28.3,24.9,22.9,20.7,14.1$, 14.1, 14.1 .

ESI-TOF LC/MS m/z calcd. for $\mathrm{C}_{18} \mathrm{H}_{30} \mathrm{NO}_{6}\left[\mathrm{M}+\mathrm{H}^{+}\right]$356.2073, found 356.2075.

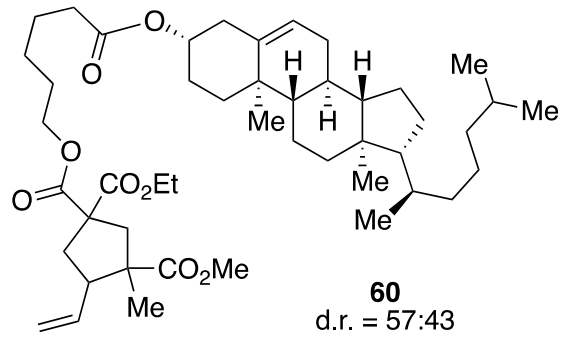

Prepared according to Procedure $\boldsymbol{C}$. Colorless oil (89.2 $\mathrm{mg}, 58 \%$ yield) as a mixture of diastereomers (d.r. $=57: 43) . \mathrm{R}_{\mathrm{f}}=0.50(10 \%$ EtOAc in hexane $)$.

${ }^{1} \mathbf{H}$ NMR $\left(400 \mathrm{MHz}, \mathrm{CDCl}_{3}\right) \delta 5.81-5.55$ (m, 1.0H), 5.36 (brs, 1.0H), $5.16-4.98(\mathrm{~m}$, $2.0 \mathrm{H}), 4.72-4.51(\mathrm{~m}, 1.0 \mathrm{H}), 4.29-3.97(\mathrm{~m}, 4.0 \mathrm{H}), 3.67(\mathrm{~s}, 1.29 \mathrm{H}), 3.60(\mathrm{~s}, 1.71 \mathrm{H}), 3.12$ $-3.01(\mathrm{~m}, 0.43 \mathrm{H}), 2.91(\mathrm{dd}, J=14.4,4.7 \mathrm{~Hz}, 0.57 \mathrm{H}), 2.83(\mathrm{dd}, J=14.3,3.2 \mathrm{~Hz}, 0.43 \mathrm{H})$, $2.58-2.44(\mathrm{~m}, 1.57 \mathrm{H}), 2.41-2.14(\mathrm{~m}, 6.0 \mathrm{H}), 2.05-0.59(\mathrm{~m}, 53.0 \mathrm{H})$. 
${ }^{13} \mathrm{C}$ NMR $\left(101 \mathrm{MHz}, \mathrm{CDCl}_{3}\right) \delta$ 176.6, 175.7, 173.1, 173.0, 172.8, 172.8, 172.8, 172.1, 172.0, 171.6, 171.5, 139.6, 136.1, 135.9, 122.6, 117.0, 116.8, 73.8, 73.8, 65.5, 65.5, 65.3, 61.7, 61.7, 61.7, 61.4, 58.9, 58.0, 56.7, 56.1, 54.6, 54.6, 53.0, 52.1, 52.0, 51.5, 51.5, 50.0, $49.8,49.7,44.8,44.7,44.6,42.3,39.7,39.5,38.9,38.8,38.1,37.4,37.0,36.6,36.2,35.8$, $34.5,34.4,31.9,31.8,28.2,28.2,28.0,27.8,25.4,25.3,24.6,24.6,24.5,24.3,23.8,22.9$, 22.9, 22.8, 22.6, 21.0, 19.3, 19.0, 18.9, 18.7, 14.1, 14.0, 11.8 .

ESI-TOF LC/MS m/z calcd. for $\mathrm{C}_{47} \mathrm{H}_{75} \mathrm{O}_{8}\left[\mathrm{M}+\mathrm{H}^{+}\right]$767.5462, found 767.5466.

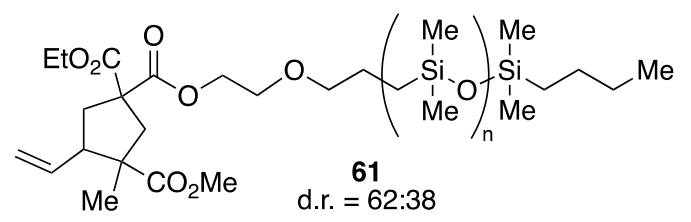

Prepared according to Procedure $\boldsymbol{C}$. Colorless oil (229.7 $\mathrm{mg}, 77 \%$ yield) as a mixture of diastereomers (d.r. $=62: 38) . \mathrm{R}_{\mathrm{f}}=0.50(10 \%$ EtOAc in hexane) .

${ }^{1} \mathbf{H}$ NMR $\left(400 \mathrm{MHz}, \mathrm{CDCl}_{3}\right) \delta 5.86-5.50(\mathrm{~m}, 1.0 \mathrm{H}), 5.15-4.91(\mathrm{~m}, 2.0 \mathrm{H}), 4.37-4.05$ $(\mathrm{m}, 4.0 \mathrm{H}), 3.77-3.54(\mathrm{~m}, 5.0 \mathrm{H}), 3.46-3.32(\mathrm{~m}, 2.0 \mathrm{H}), 3.16-3.02(\mathrm{~m}, 0.38 \mathrm{H}), 2.94(\mathrm{~d}$, $J=14.4 \mathrm{~Hz}, 0.62 \mathrm{H}), 2.86(\mathrm{dd}, J=14.3,10.6 \mathrm{~Hz}, 0.38 \mathrm{H}), 2.61-2.47(\mathrm{~m}, 1.62 \mathrm{H}), 2.44-$ $2.16(\mathrm{~m}, 2.0 \mathrm{H}), 1.66-1.50(\mathrm{~m}, 2.0 \mathrm{H}), 1.38-1.19(\mathrm{~m}, 9.0 \mathrm{H}), 1.10(\mathrm{~d}, J=1.4 \mathrm{~Hz}, 1.0 \mathrm{H})$, $0.92-0.82(\mathrm{~m}, 3.0 \mathrm{H}), 0.61-0.45(\mathrm{~m}, 4.0 \mathrm{H}), 0.28--0.17(\mathrm{~m}, 74.0 \mathrm{H})$.

${ }^{13} \mathbf{C}$ NMR $\left(101 \mathrm{MHz}, \mathrm{CDCl}_{3}\right) \delta 175.5,174.7,174.6,172.0,171.9,171.1,170.8,170.6$, 170.3, 135.1, 134.9, 134.8, 116.0, 116.0, 115.8, 73.1, 73.0, 67.2, 67.2, 63.8, 63.7, 63.6, $60.7,60.7,60.6,60.4,57.9,57.8,57.0,53.6,53.5,52.0,52.0,51.0,50.9,50.4,48.7,48.7$, 43.7, 43.6, 43.6, 37.8, 36.4, 25.3, 24.4, 22.4, 21.9, 21.8, 17.9, 17.8, 16.9, 13.1, 13.1, 13.0, $12.9,12.8,0.4,0.1,0.00,-0.4,-0.9,-1.0$.

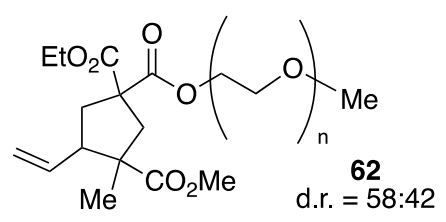

Prepared according to Procedure $\boldsymbol{C}$. Colorless oil (108.3 $\mathrm{mg}, 75 \%$ yield) as a mixture of diastereomers (d.r. $=58: 42)$. $\mathrm{R}_{\mathrm{f}}=0.30(30 \%$ acetone in hexane).

${ }^{1} \mathbf{H}$ NMR $\left(400 \mathrm{MHz}, \mathrm{CDCl}_{3}\right) \delta 5.82-5.54(\mathrm{~m}, 1.0 \mathrm{H}), 5.15-4.90(\mathrm{~m}, 2.0 \mathrm{H}), 4.36-4.09$ $(\mathrm{m}, 4.0 \mathrm{H}), 3.81-3.48(\mathrm{~m}, 44.0 \mathrm{H}), 3.35(\mathrm{~s}, 3.0 \mathrm{H}), 3.11-2.99(\mathrm{~m}, 0.42 \mathrm{H}), 2.90(\mathrm{~d}, J=$ $14.4 \mathrm{~Hz}, 0.58 \mathrm{H}), 2.83(\mathrm{dd}, J=14.3,5.6 \mathrm{~Hz}, 0.42 \mathrm{H}), 2.60-2.43(\mathrm{~m}, 1.58 \mathrm{H}), 2.41-2.14$ $(\mathrm{m}, 2.0 \mathrm{H}), 1.35-1.17(\mathrm{~m}, 4.74 \mathrm{H}), 1.08(\mathrm{~d}, J=1.8 \mathrm{~Hz}, 1.26 \mathrm{H})$.

${ }^{13} \mathrm{C}$ NMR $\left(101 \mathrm{MHz}, \mathrm{CDCl}_{3}\right) \delta 176.5,175.6,175.6,172.9,172.8,172.0,171.8,171.8$, $171.5,171.3,136.0,135.9,135.8,117.0,117.0,116.8,71.9,70.6,70.6,70.5,70.5,68.8$, $68.8,68.8,64.7,64.7,64.6,64.5,61.7,61.7,61.7,61.4,59.0,58.8,58.8,57.9,57.9,54.6$, 53.0, 53.0, 52.0, 52.0, 51.9, 51.5, 49.7, 49.7, 44.7, 44.6, 44.6, 38.8, 37.3, 37.3, 22.9, 22.77, $18.9,18.8,14.0,14.0$. 


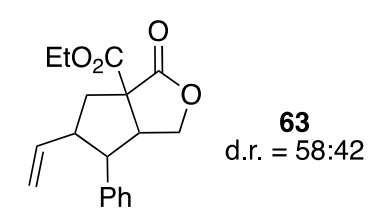

Prepared according to Procedure $\boldsymbol{C}$. Colorless oil $(25.7 \mathrm{mg}, 43 \%$ yield) as a mixture of diastereomers (d.r. $=58: 42) . \mathrm{R}_{\mathrm{f}}=0.30(10 \%$ EtOAc in hexane $)$.

${ }^{1} \mathbf{H}$ NMR $\left(400 \mathrm{MHz}, \mathrm{CDCl}_{3}\right) \delta 7.33-6.91(\mathrm{~m}, 5.0 \mathrm{H}), 5.56-5.43(\mathrm{~m}, 0.42 \mathrm{H}), 5.32-5.15$ $(\mathrm{m}, 0.58 \mathrm{H}), 4.93-4.63(\mathrm{~m}, 2.0 \mathrm{H}), 4.58-4.07(\mathrm{~m}, 4.0 \mathrm{H}), 3.77-1.69(\mathrm{~m}, 5.0 \mathrm{H}), 1.35-$ $1.05(\mathrm{~m}, 3.0 \mathrm{H})$.

${ }^{13}$ C NMR (101 MHz, CDCl3) $\delta 176.5,176.4,171.3,169.7,169.4,169.0,138.9,138.9$, $138.6,137.7,137.1,136.5,128.9,128.5,128.4,128.1,127.8,127.5,127.0,126.8,116.5$, $116.4,115.1,72.0,69.8,69.4,62.5,62.5,62.4,61.6,60.7,59.3,57.7,56.6,55.9,55.1,52.5$, 51.8, 50.4, 48.7, 43.4, 38.1, 37.5, 33.6, 14.2, 14.1 .

DART-TOF LC/MS m/z calcd. for $\mathrm{C}_{18} \mathrm{H}_{24} \mathrm{NO}_{4}\left[\mathrm{M}+\mathrm{NH}_{4}{ }^{+}\right]$318.1705, found 318.1703.

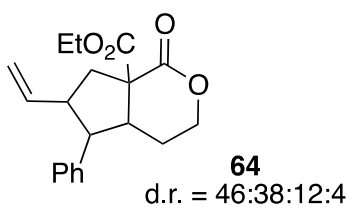

Prepared according to Procedure $\boldsymbol{C}$. Colorless oil (45.1 mg, 72\% yield) as a mixture of diastereomers (d.r. $=46: 38: 12: 4) . \mathrm{R}_{\mathrm{f}}=0.30(10 \%$ EtOAc in hexane $)$.

${ }^{1} \mathbf{H}$ NMR $\left(400 \mathrm{MHz}, \mathrm{CDCl}_{3}\right) \delta 7.35-6.96(\mathrm{~m}, 5.0 \mathrm{H}), 5.82(\mathrm{ddd}, J=17.5,10.2,7.8 \mathrm{~Hz}$, $0.04 \mathrm{H}$ ), 5.51 (ddd, $J=17.4,10.5,7.1 \mathrm{~Hz}, 0.46 \mathrm{H}$ ), 5.40 (ddd, $J=17.0,10.4,8.0 \mathrm{~Hz}$, $0.38 \mathrm{H}), 5.23(\mathrm{ddd}, J=17.2,10.2,8.1 \mathrm{~Hz}, 0.12 \mathrm{H}), 4.490-4.23(\mathrm{~m}, 2.0 \mathrm{H}), 4.48-4.07$ (m, $4.0 \mathrm{H}), 3.77-2.71(\mathrm{~m}, 3.0 \mathrm{H}), 2.69-1.70(\mathrm{~m}, 3.0 \mathrm{H}), 1.63-1.42(\mathrm{~m}, 1.0 \mathrm{H}), 1.32-1.20$ $(\mathrm{m}, 3.0 \mathrm{H})$.

${ }^{13}$ C NMR (101 MHz, CDC13) $\delta 172.1,171.7,171.5,171.4,171.2,170.1,139.9,139.5$, $138.9,138.9,137.8,137.6,128.7,128.7,128.6,128.3,128.3,127.7,127.5,127.2,126.8$, $126.6,116.0,115.9,114.6,67.9,67.2,67.0,62.6,62.4,62.1,59.5,59.1,59.0,57.6,56.9$, 50.0, 50.0, 49.5, 47.9, 46.5, 45.2, 44.2, 40.7, 39.7, 37.0, 28.0, 27.9, 22.8, 14.1, 14.0, 14.0.

DART-TOF LC/MS m/z calcd. for $\mathrm{C}_{19} \mathrm{H}_{26} \mathrm{NO}_{4}\left[\mathrm{M}+\mathrm{NH}_{4}{ }^{+}\right] 332.1862$, found 332.1858.

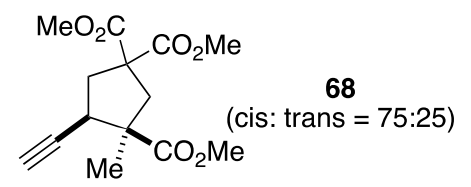

Prepared according to Procedure $\boldsymbol{C}$. Colorless oil (37.3 $\mathrm{mg}, 66 \%$ yield) as a mixture of inseparable diastereomers (cis : trans $=75: 25)$. $\mathrm{R}_{\mathrm{f}}=0.30$ (20\% EtOAc in hexane).

${ }^{1} \mathbf{H}$ NMR (400 MHz, $\left.\mathrm{CDCl}_{3}\right) \delta 3.74(\mathrm{~s}, 2.25 \mathrm{H}), 3.72(\mathrm{~s}, 3.00 \mathrm{H}), 3.71$ (s, 0.75H), 3.70 (s, $0.75 \mathrm{H}), 3.67(\mathrm{~s}, 2.25 \mathrm{H}), 3.34(\mathrm{ddd}, J=10.0,7.2,2.5 \mathrm{~Hz}, 0.25 \mathrm{H}), 2.99$ (d, $J=14.5 \mathrm{~Hz}$, $0.75 \mathrm{H}), 2.81-2.66(\mathrm{~m}, 2 \mathrm{H}), 2.60(\mathrm{dd}, J=13.0,6.9 \mathrm{~Hz}, 0.75 \mathrm{H}), 2.45(\mathrm{~d}, J=14.3 \mathrm{~Hz}$, $0.25 \mathrm{H}), 2.33(\mathrm{dd}, J=13.8,10.2 \mathrm{~Hz}, 0.25 \mathrm{H}), 2.20-2.10(\mathrm{~m}, 1.75 \mathrm{H}), 1.36(\mathrm{~s}, 2.25 \mathrm{H}), 1.33$ $(\mathrm{s}, 0.75 \mathrm{H})$. 
${ }^{13} \mathbf{C}$ NMR $\left(101 \mathrm{MHz}, \mathrm{CDCl}_{3}\right) \delta 175.8,175.0,173.0,172.0,171.7,171.4,82.1,81.7,72.1$, 71.5, 58.9, 58.1, 53.7, 53.1, 53.0, 53.0, 52.9, 52.4, 51.8, 43.8, 43.7, 41.4, 39.7, 39.2, 37.9, 29.7, 23.0, 20.5.

DART-TOF LC/MS m/z calcd. for $\mathrm{C}_{14} \mathrm{H}_{22} \mathrm{NO}_{6}\left[\mathrm{M}+\mathrm{NH}_{4}{ }^{+}\right] 300.1447$, found 300.1446 .

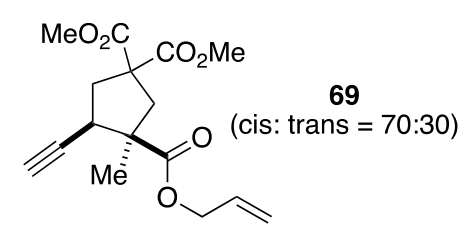

Prepared according to Procedure $\boldsymbol{C}$. Colorless oil (29.8 $\mathrm{mg}$, $48 \%$ yield) as a mixture of inseparable diastereomers (cis : trans $=70: 30)$. $R_{\mathrm{f}}=0.35$ (20\% EtOAc in hexane).

${ }^{1} \mathbf{H}$ NMR $\left(400 \mathrm{MHz}, \mathrm{CDCl}_{3}\right) \delta 5.93-5.76(\mathrm{~m}, 1.0 \mathrm{H}), 5.26(\mathrm{~d}, J=17.2,1.0 \mathrm{H}), 5.20-5.10$ $(\mathrm{m}, 1.0 \mathrm{H}), 4.59-4.44(\mathrm{~m}, 2.0 \mathrm{H}), 3.75-3.58(\mathrm{~m}, 6.0 \mathrm{H}), 3.30(\mathrm{ddd}, J=10.0,7.2,2.5 \mathrm{~Hz}$, $0.30 \mathrm{H}), 2.96(\mathrm{~d}, J=14.5 \mathrm{~Hz}, 0.70 \mathrm{H}), 2.77-2.62(\mathrm{~m}, 2.0 \mathrm{H}), 2.55(\mathrm{dd}, J=13.0,6.8 \mathrm{~Hz}$, $0.70 \mathrm{H}), 2.41(\mathrm{~d}, J=14.4 \mathrm{~Hz}, 0.30 \mathrm{H}), 2.28(\mathrm{dd}, J=13.7,10.3 \mathrm{~Hz}, 0.30 \mathrm{H}), 2.18-2.02(\mathrm{~m}$, $1.70 \mathrm{H}), 1.33$ (s, 2.10H), $1.30(\mathrm{~s}, 0.90 \mathrm{H})$.

${ }^{13} \mathrm{C}$ NMR $\left(101 \mathrm{MHz}, \mathrm{CDCl}_{3}\right) \delta 174.9,174.2,173.1,171.9,171.7,171.4,132.1,131.9$, $118.2,118.0,82.1,81.7,72.1,71.7,65.7,65.6,58.9,58.1,53.7,53.1,53.1,53.0,52.9$, 51.9, 43.8, 43.7, 41.4, 39.8, 39.3, 37.9, 29.7, 23.1, 20.5.

DART-TOF LC/MS m/z calcd. for $\mathrm{C}_{16} \mathrm{H}_{24} \mathrm{NO}_{6}\left[\mathrm{M}+\mathrm{NH}_{4}{ }^{+}\right] 326.1604$, found 326.1602 . 


\section{Reference}

1. Fujihashi, M.; Ishida, T.; Kuroda, S.; Kotra, L. P.; Pai, E. F.; Miki, K. Substrate Distortion Contributes to the Catalysis of Orotidine 5'-Monophosphate Decarboxylase. J. Am. Chem. Soc. 2013, 135, 17432-17443.

2. Ding, S.; Cao, S.; Zhu, A.; Shi, G. Wettability Switching of Electrode for Signal Amplification: Conversion of Conformational Change of Stimuli-Responsive Polymer into Enhanced Electrochemical Chiral Analysis. Anal. Chem. 2016, 88, 12219 -12226.

3. (a) Hashimoto, T.; Kawamata, Y.; Maruoka, K. An organic thiyl radical catalyst for enantioselective cyclization. Nat. Chem. 2014, 6, 702-705. (b) Emmett, M. R.; Kerr, M. A. Nucleophilic ring opening of cyclopropane hemimalonates using internal Brønsted acid activation. Org. Lett. 2011, 13, 4180-4183. (c) Seiji, Y.; Akiko, A.; Naoko, K.; Keiko, H.; Hajime, Y.; Yoshiro, H. Bull. Chem. Soc. Jpn. 1997, 70, 215-2219. (d) Pierre L. Beaulieu, P. L.; Gillard, J.; Bailey, M. D.; Boucher, C.; Duceppe, J.-C.; Simoneau, B.; Wang, X.-J.; Zhang, L.; Grozinger, K.; Houpis, I.; Farina, V.; Heimroth, H.; Krueger, T.; Schnaubelt, J. Synthesis of (1R,2S)-1-amino-2-vinylcyclopropanecarboxylic acid vinyl-ACCA derivatives: key intermediates for the preparation of inhibitors of the hepatitis C virus NS3 protease. J. Org. Chem. 2005, 70, 5869-5879. (e) Umeyama, T.; Naka, K.; Chujo, Y. Radical copolymerization of cyclic diarsine with vinyl monomers. J. Polym. Sci., Part A: Polym. Chem. 2004, 42, 3023-3028. 


\section{NMR Spectra}

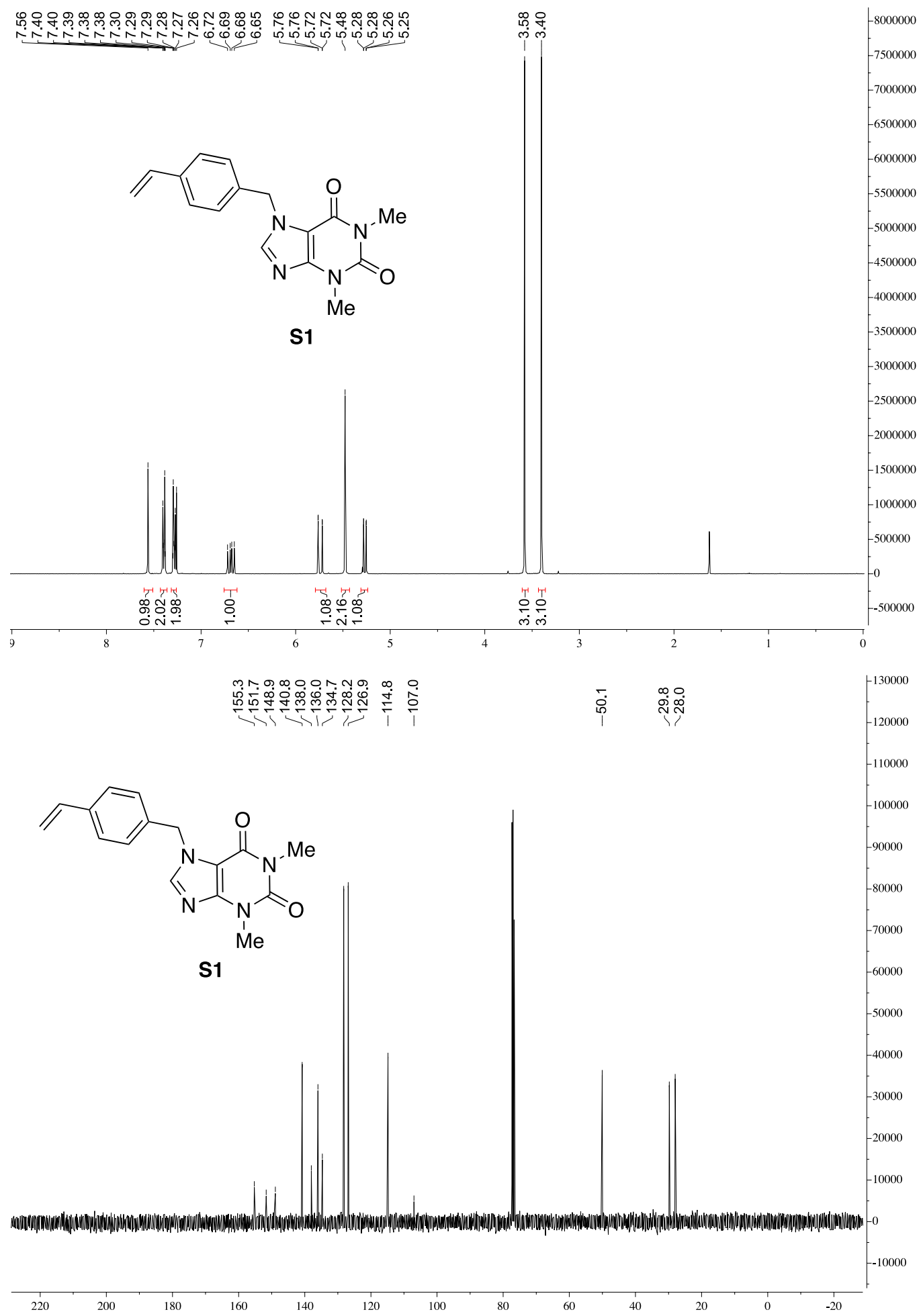




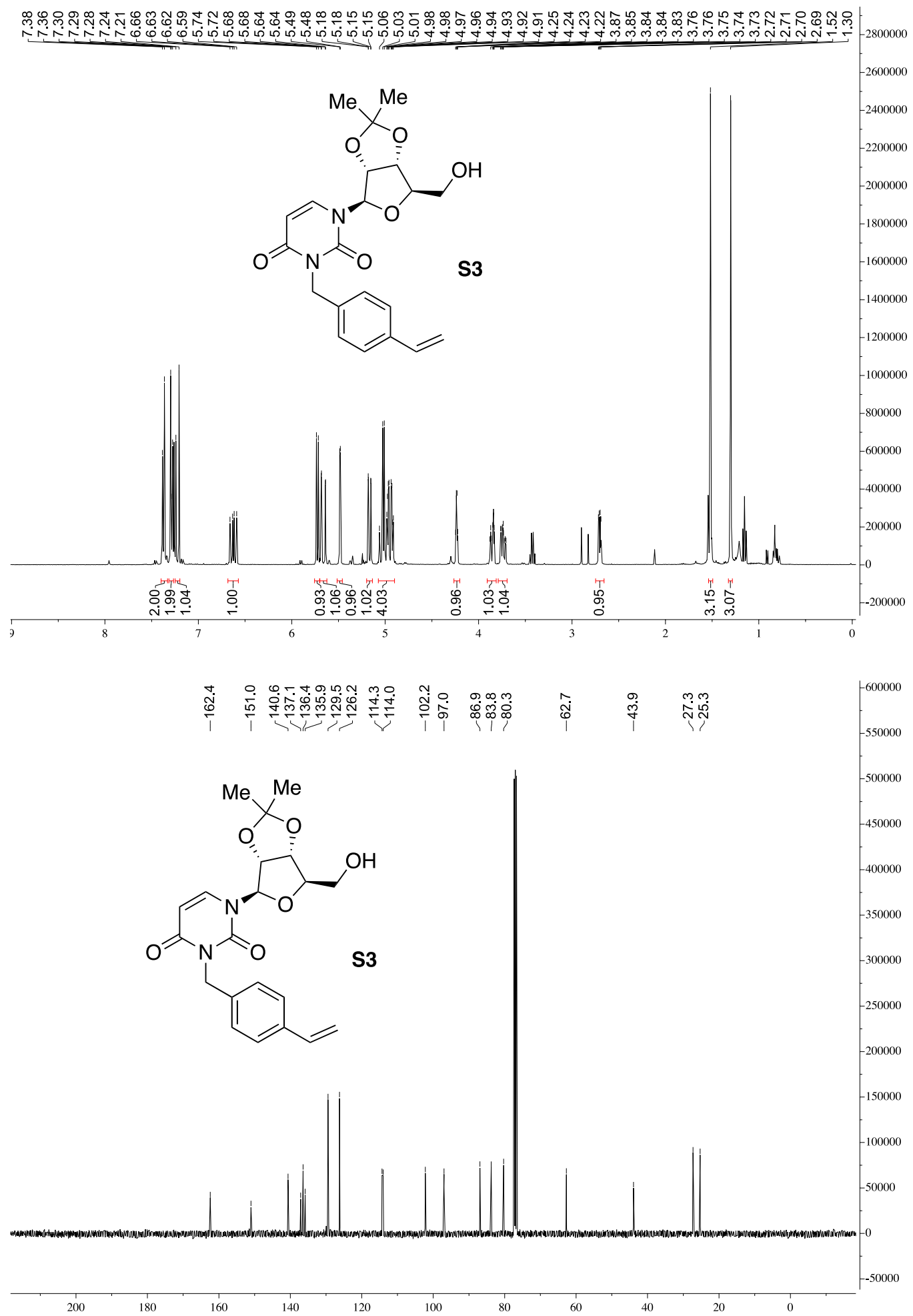



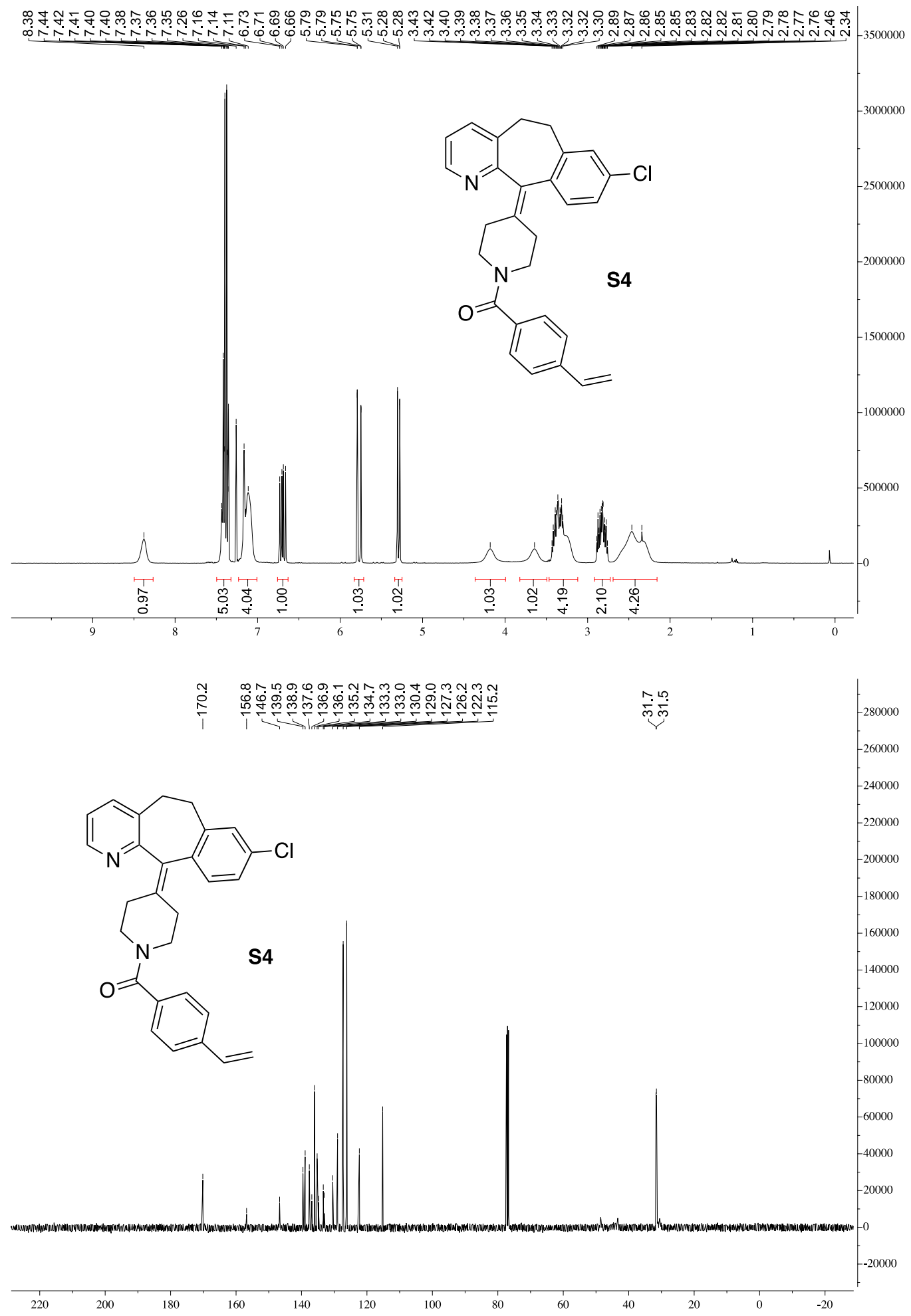


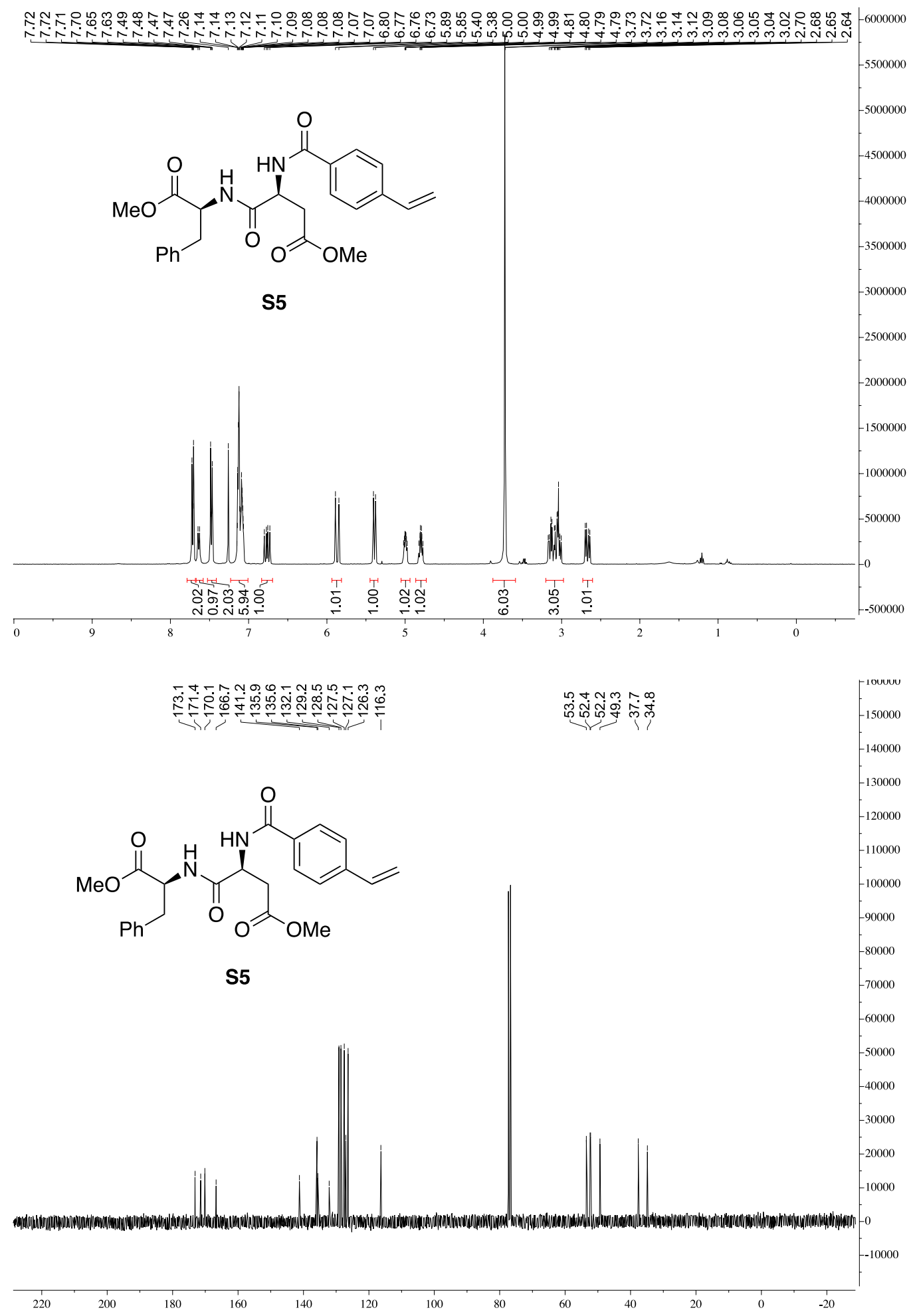




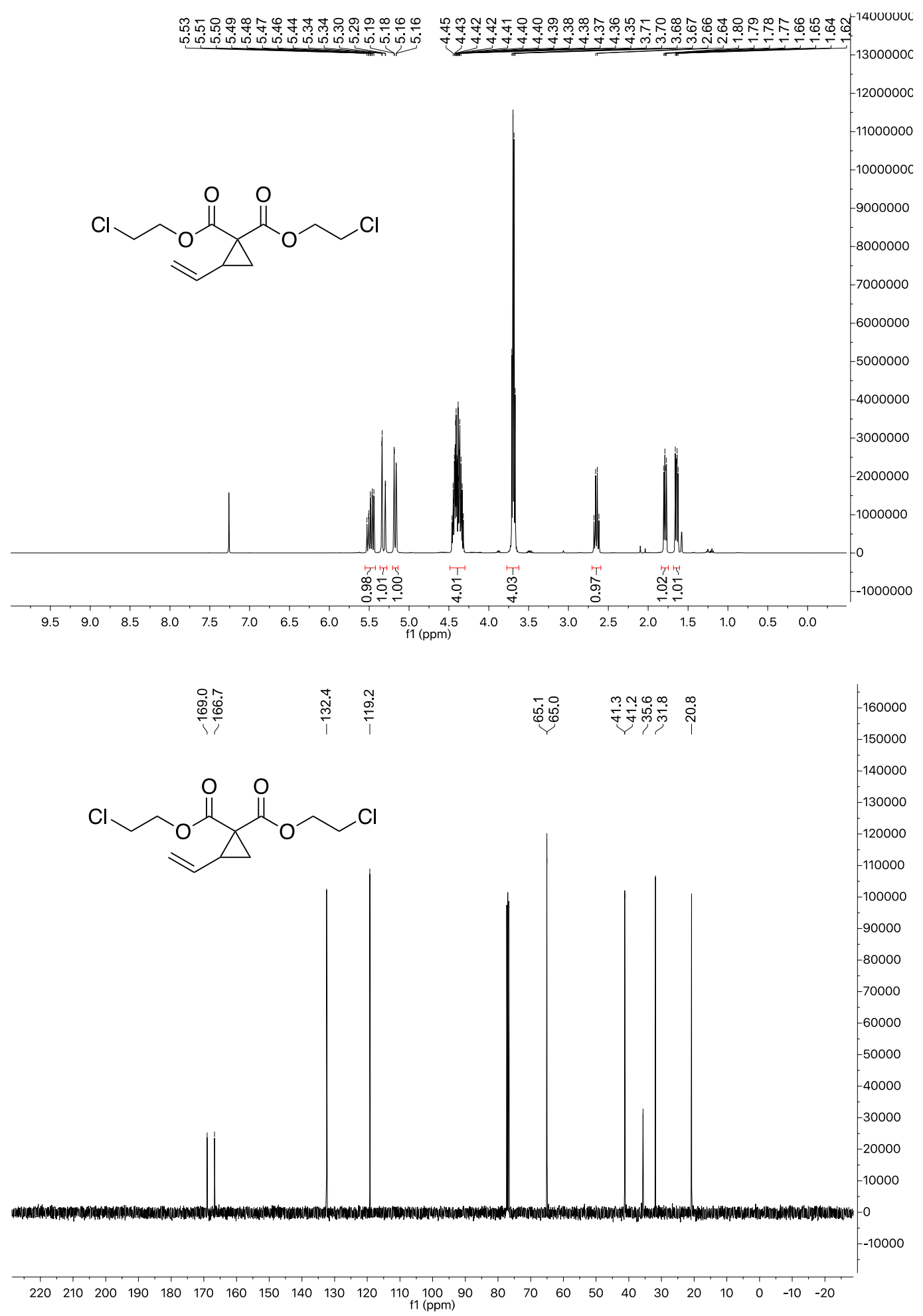




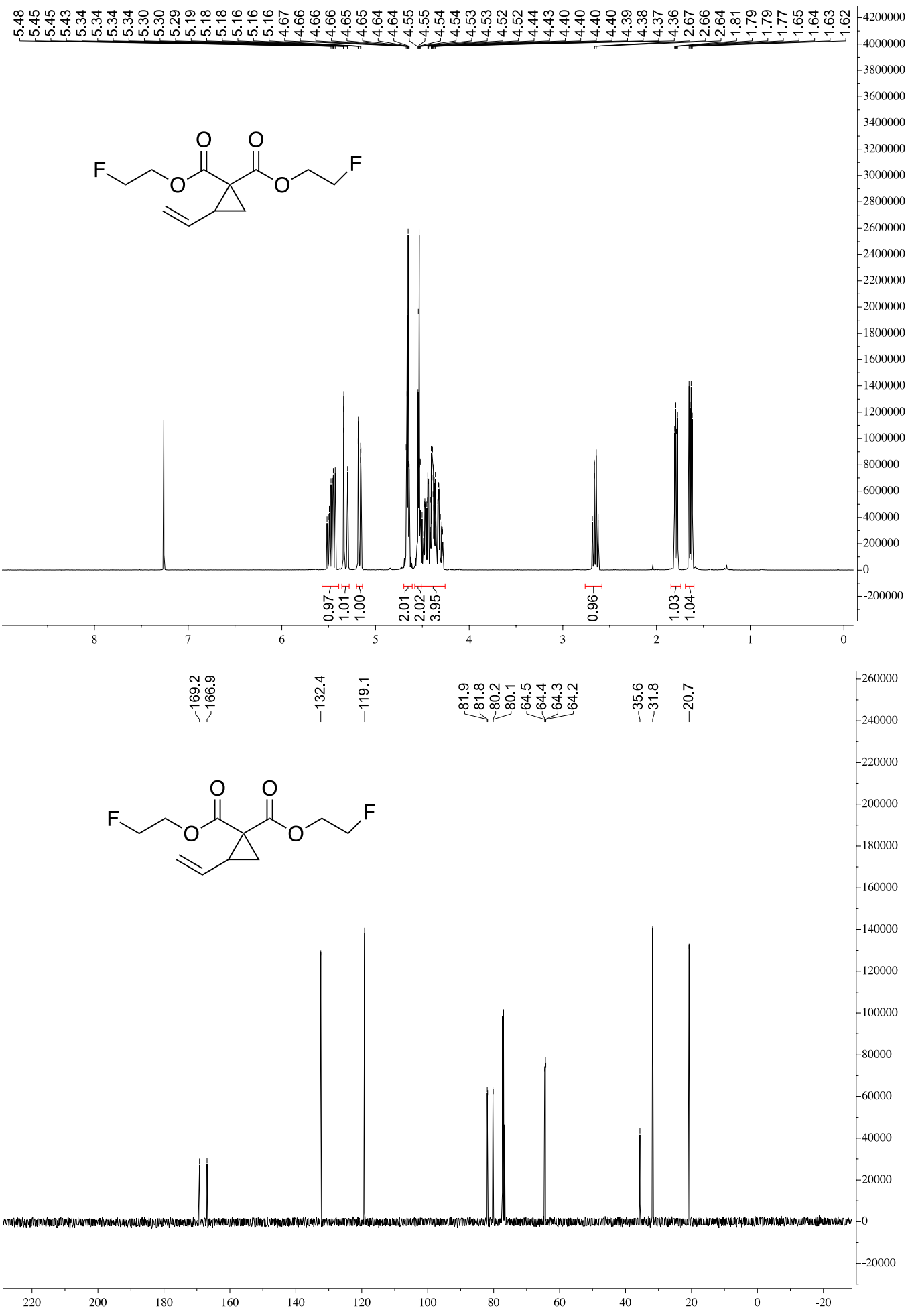




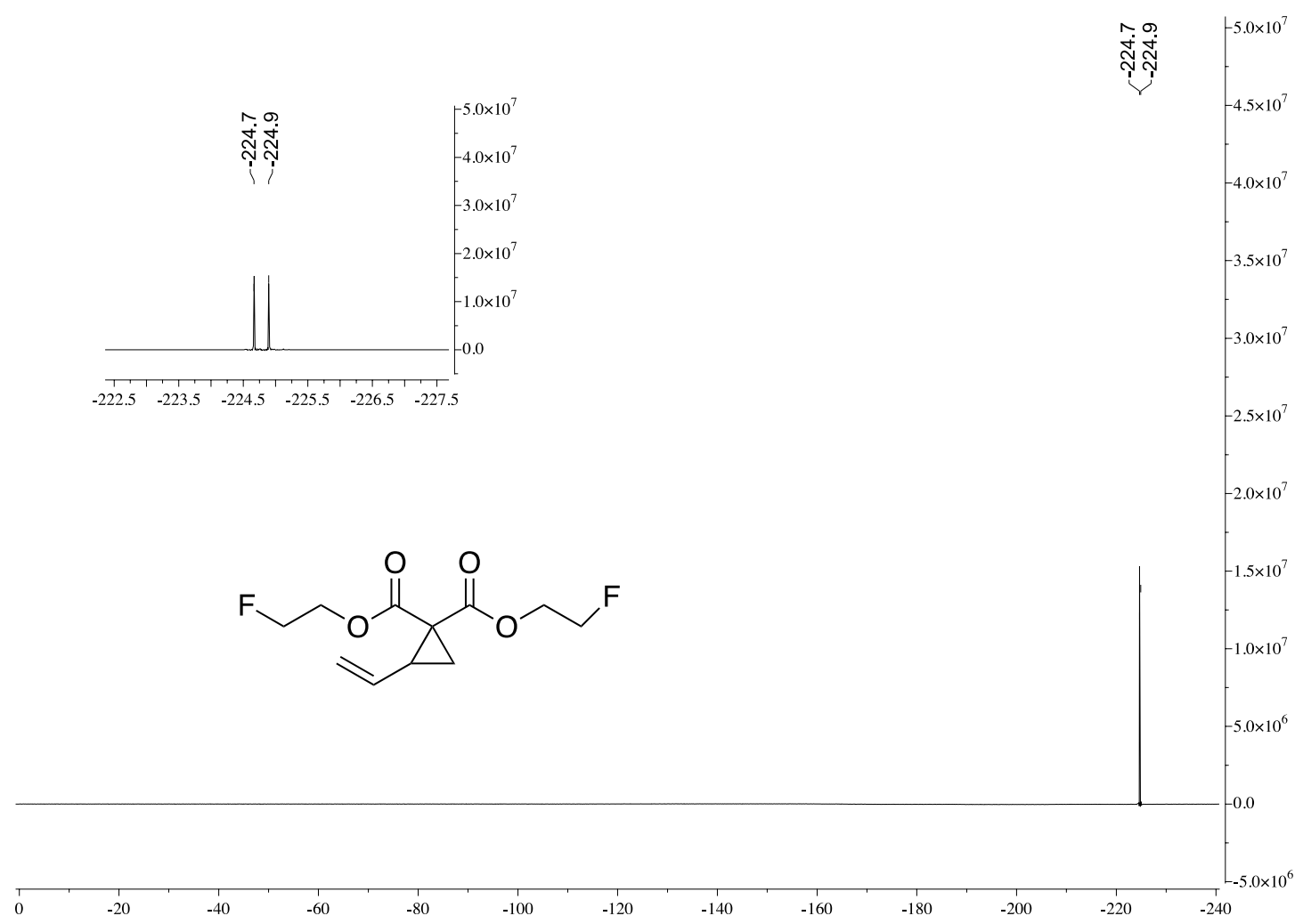



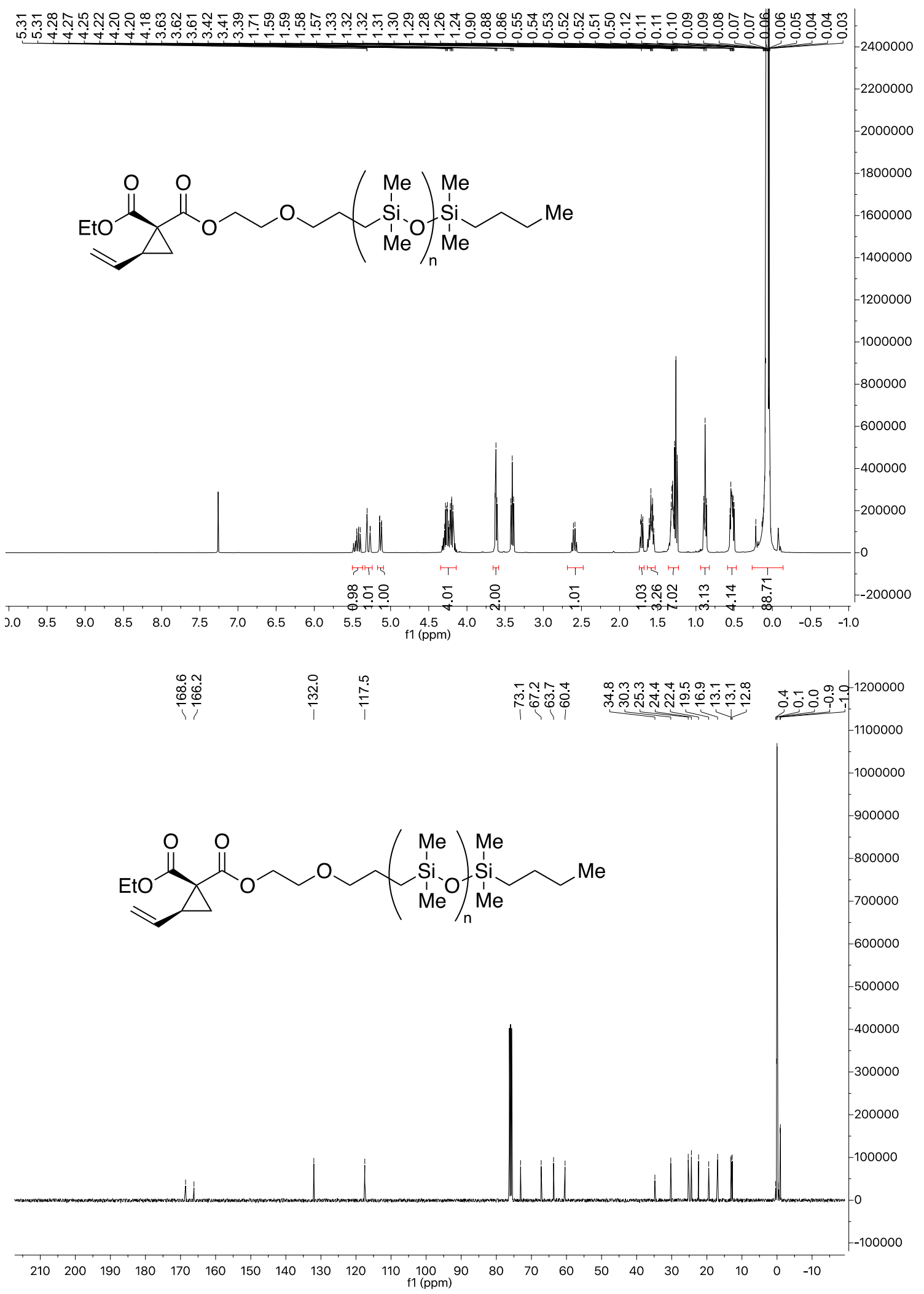


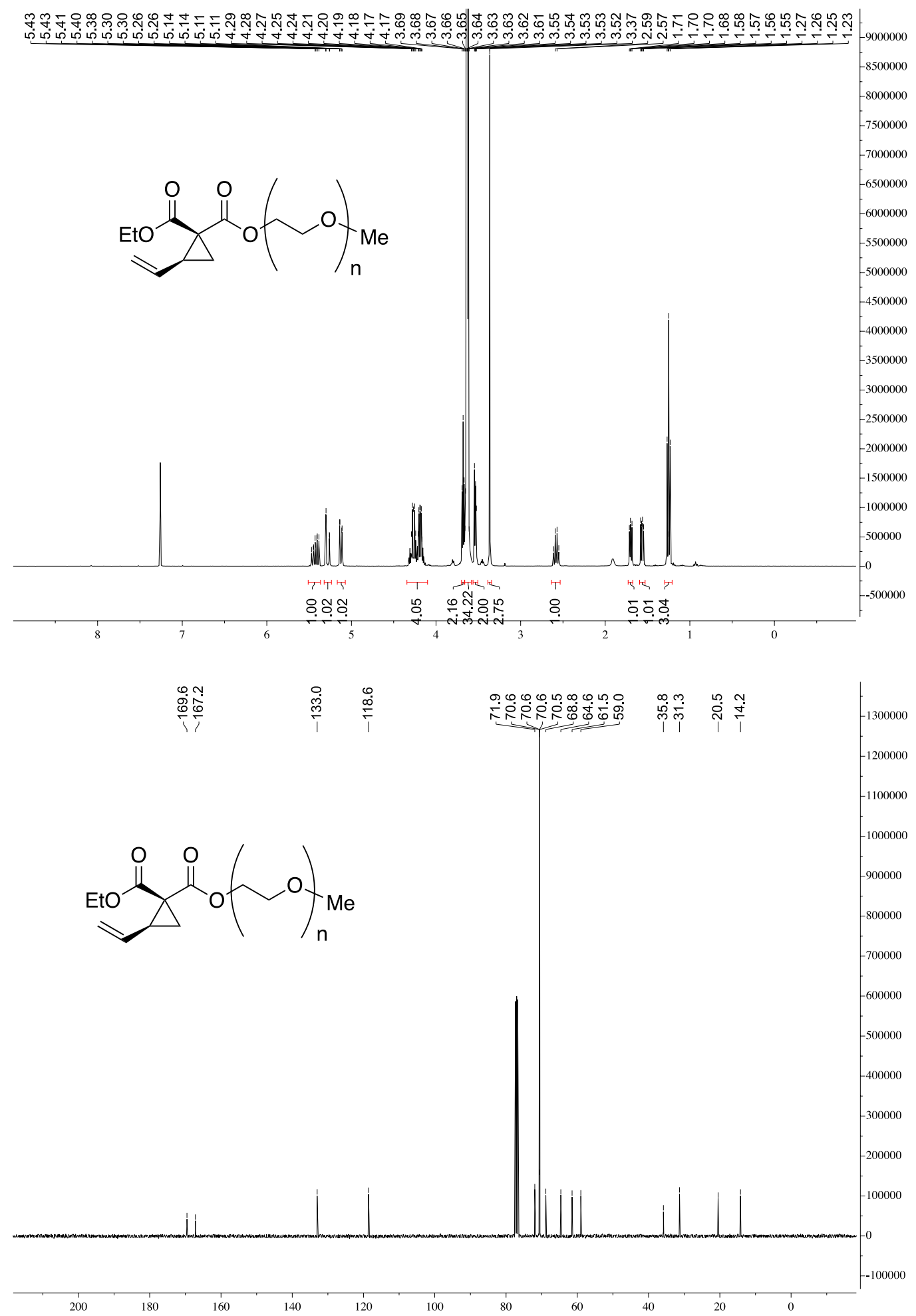




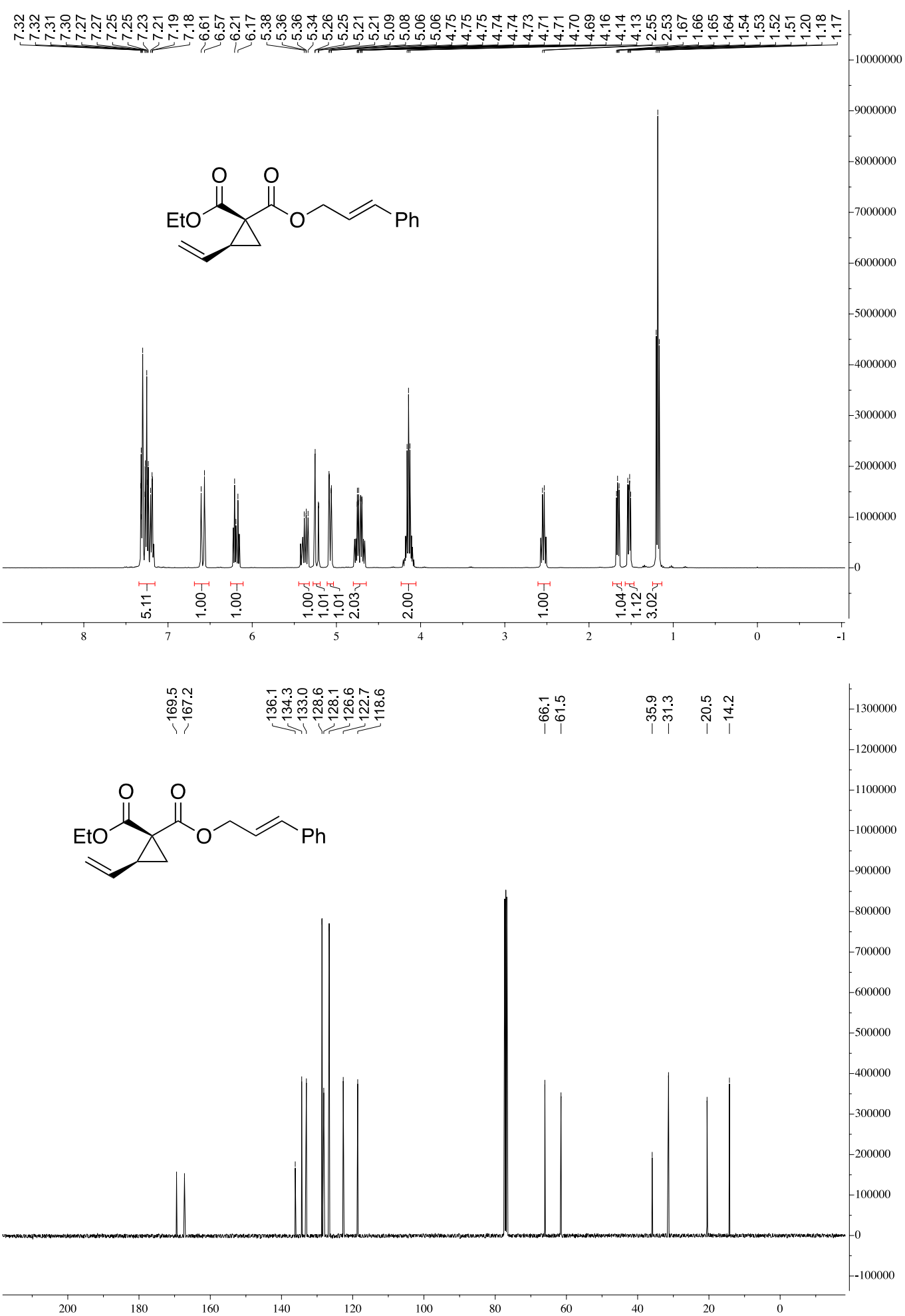




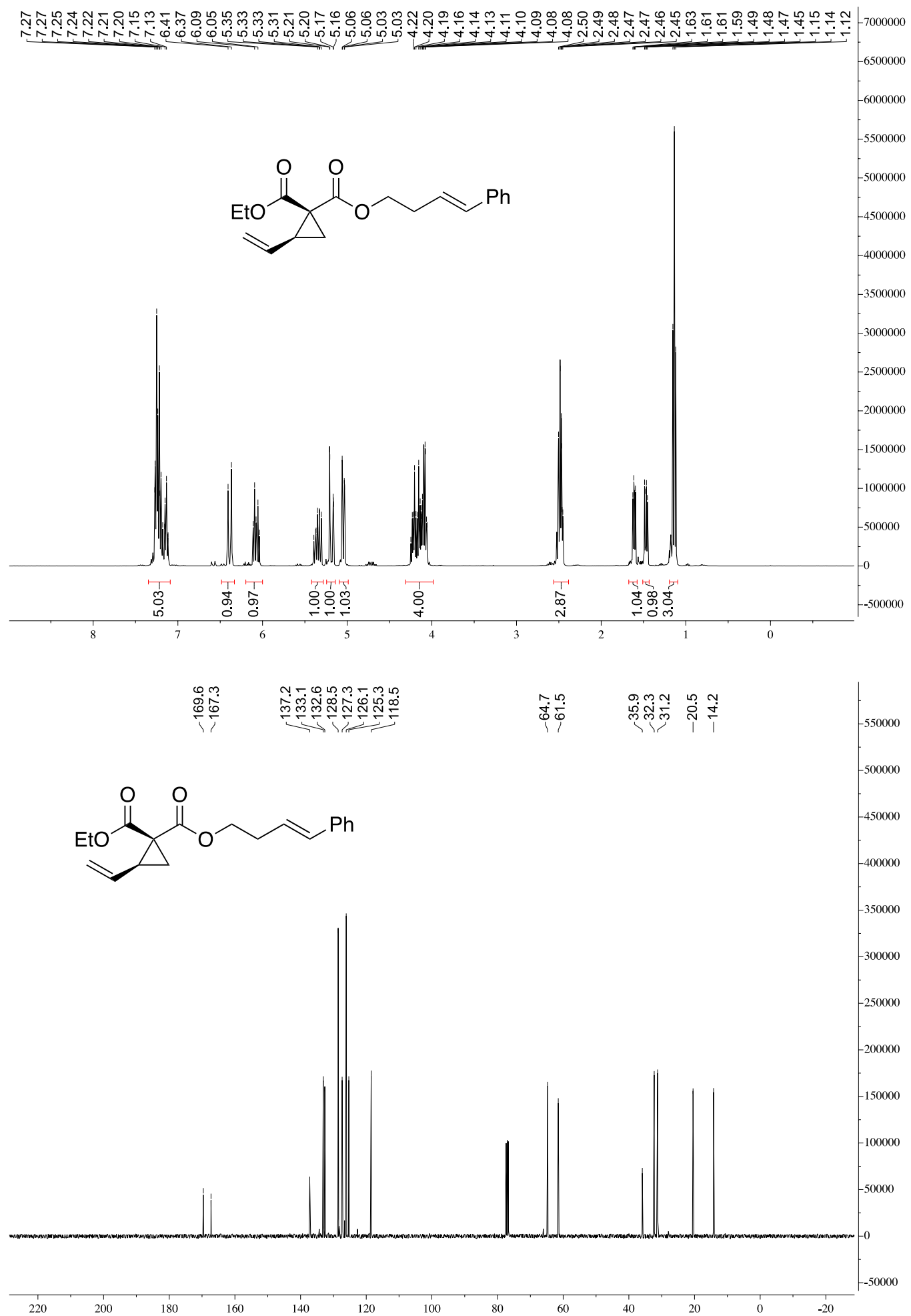




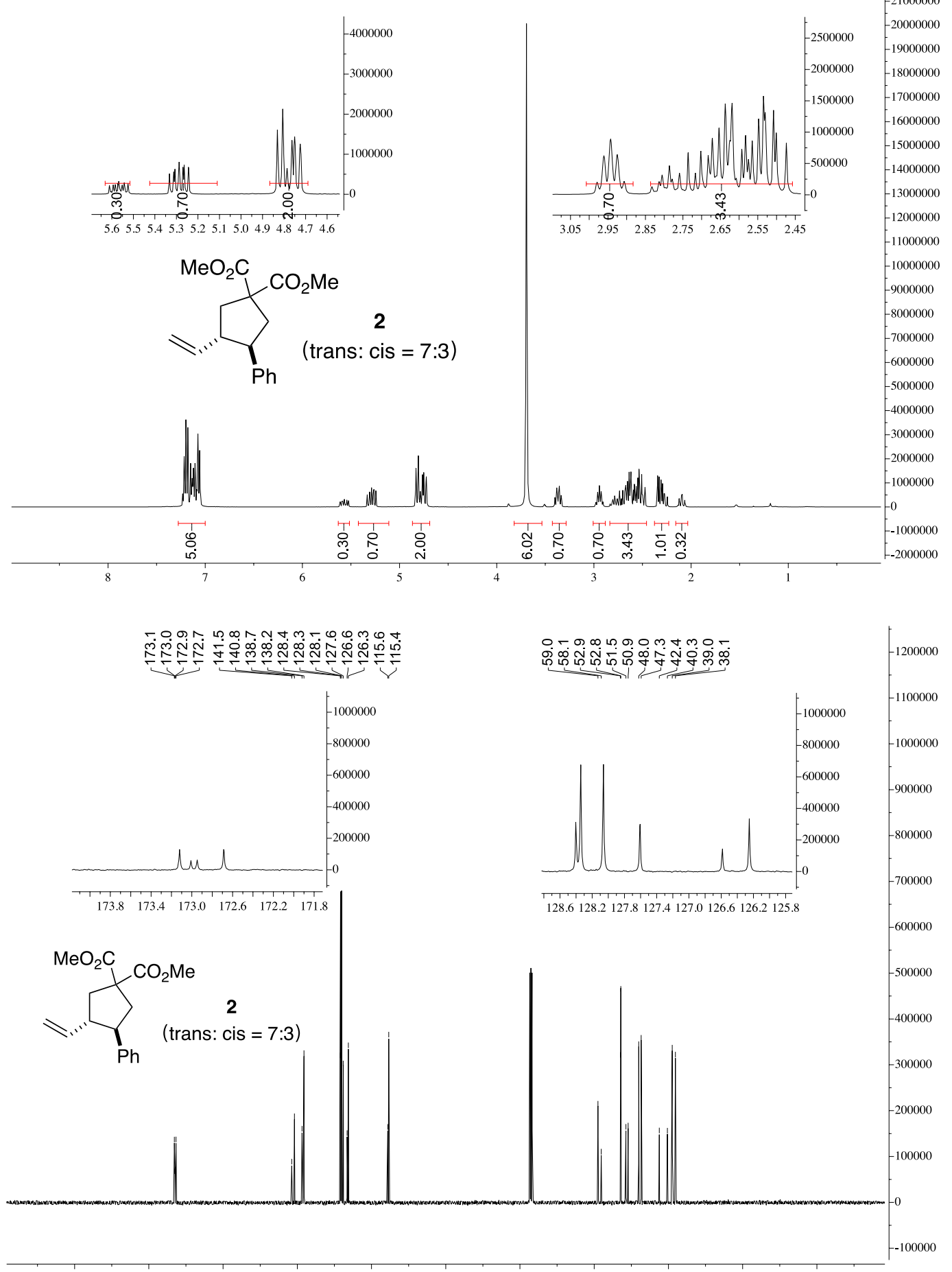

$200 \quad 180 \quad 160 \quad 140 \quad 120 \quad 100$

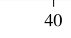



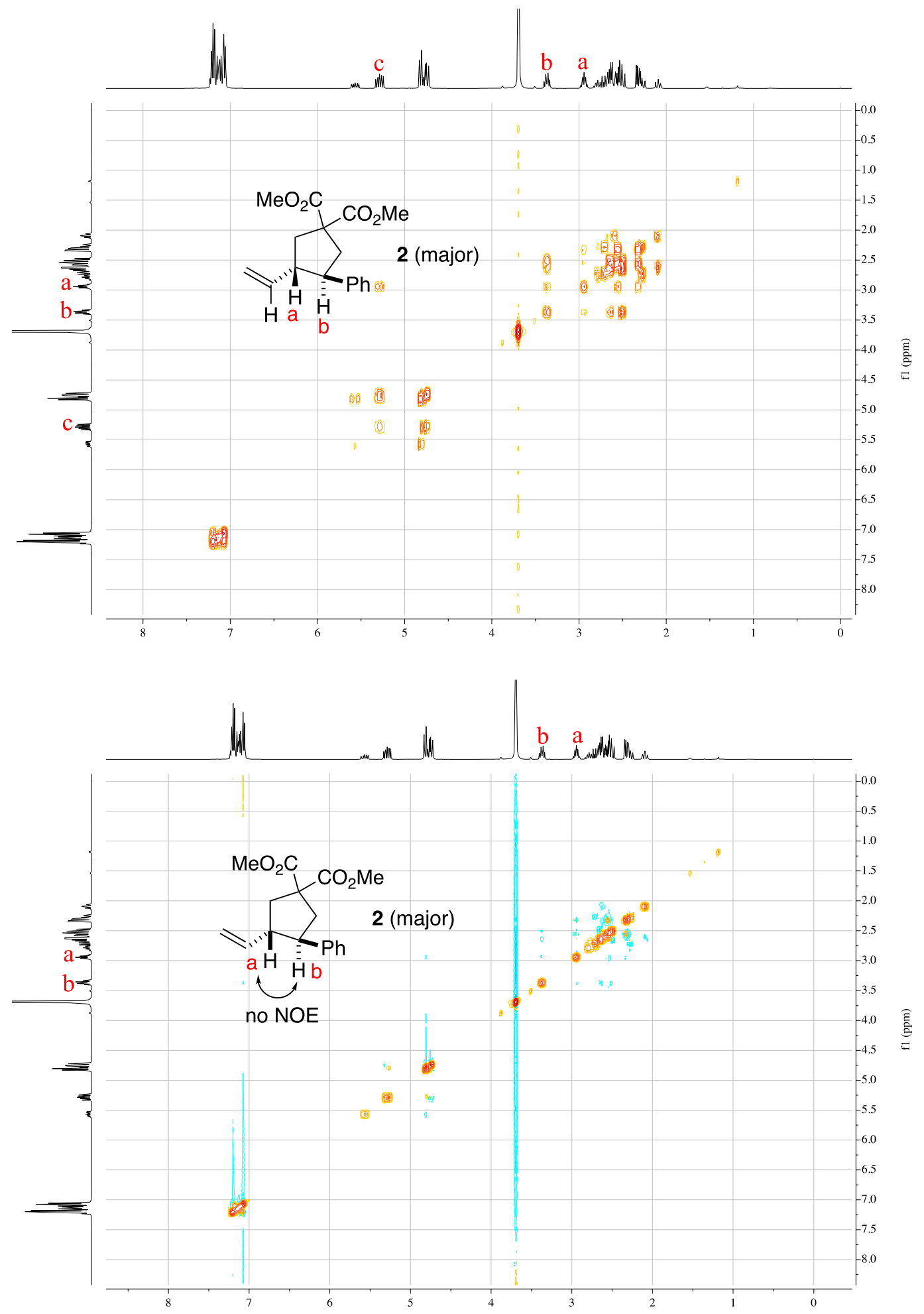

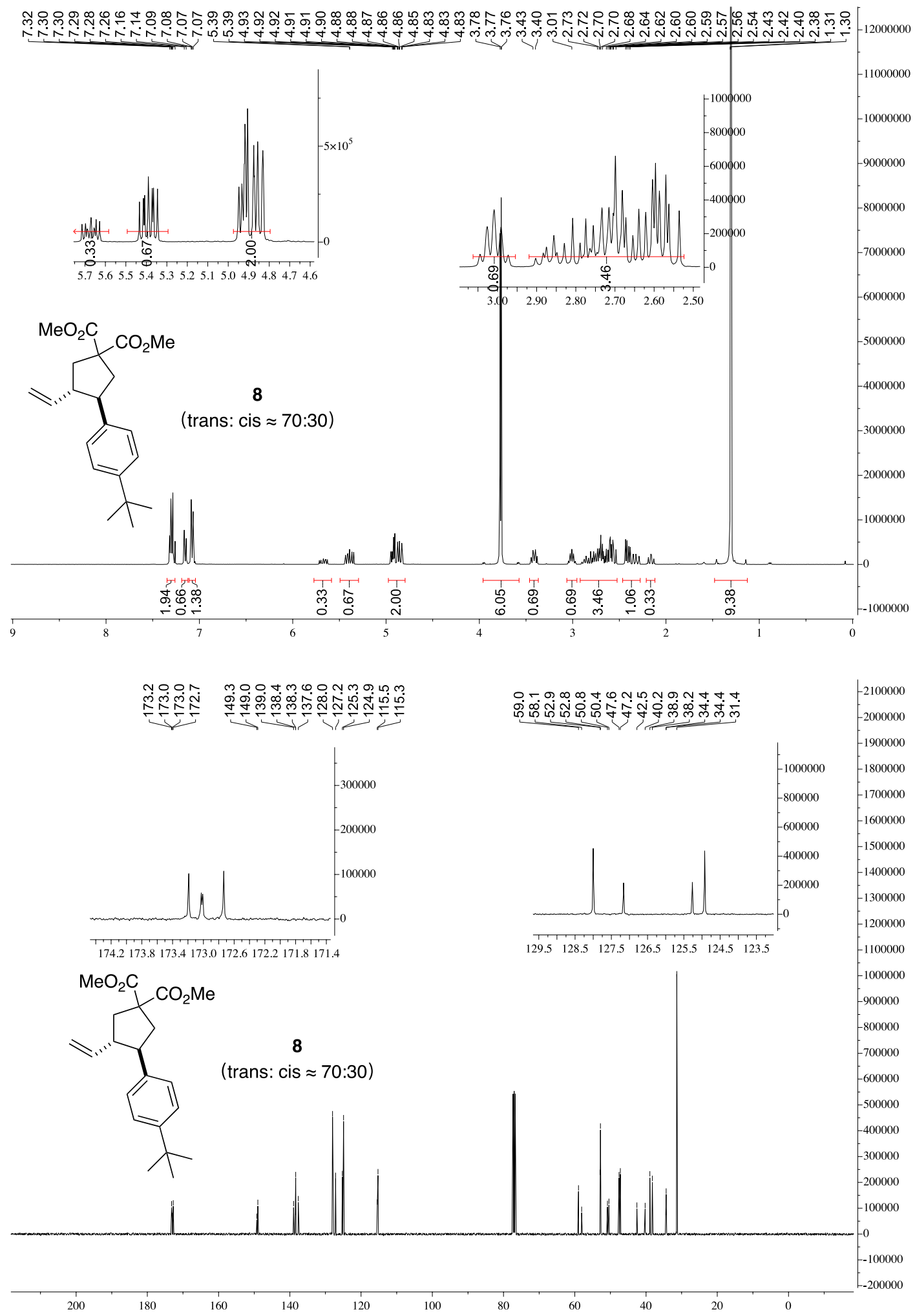


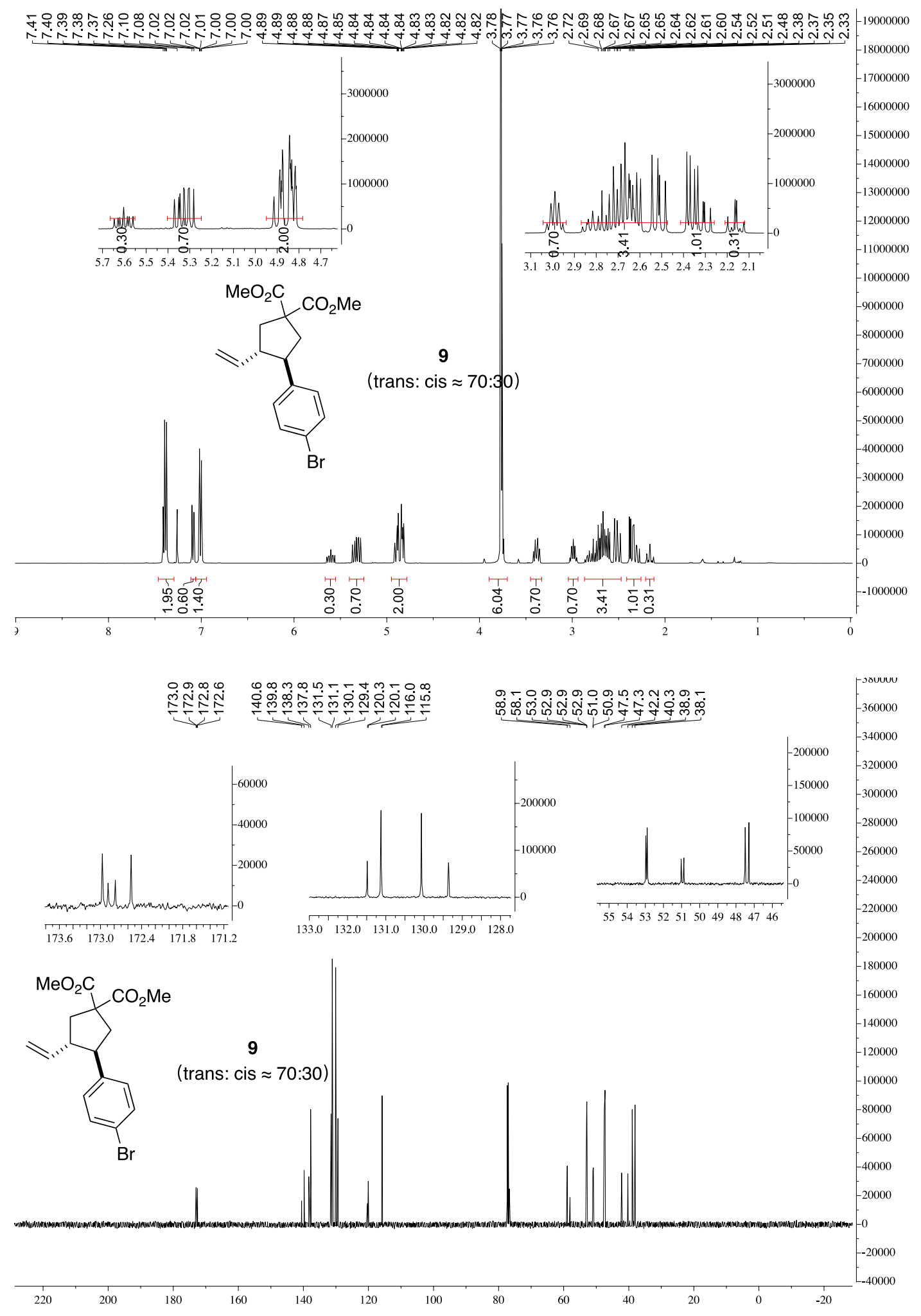




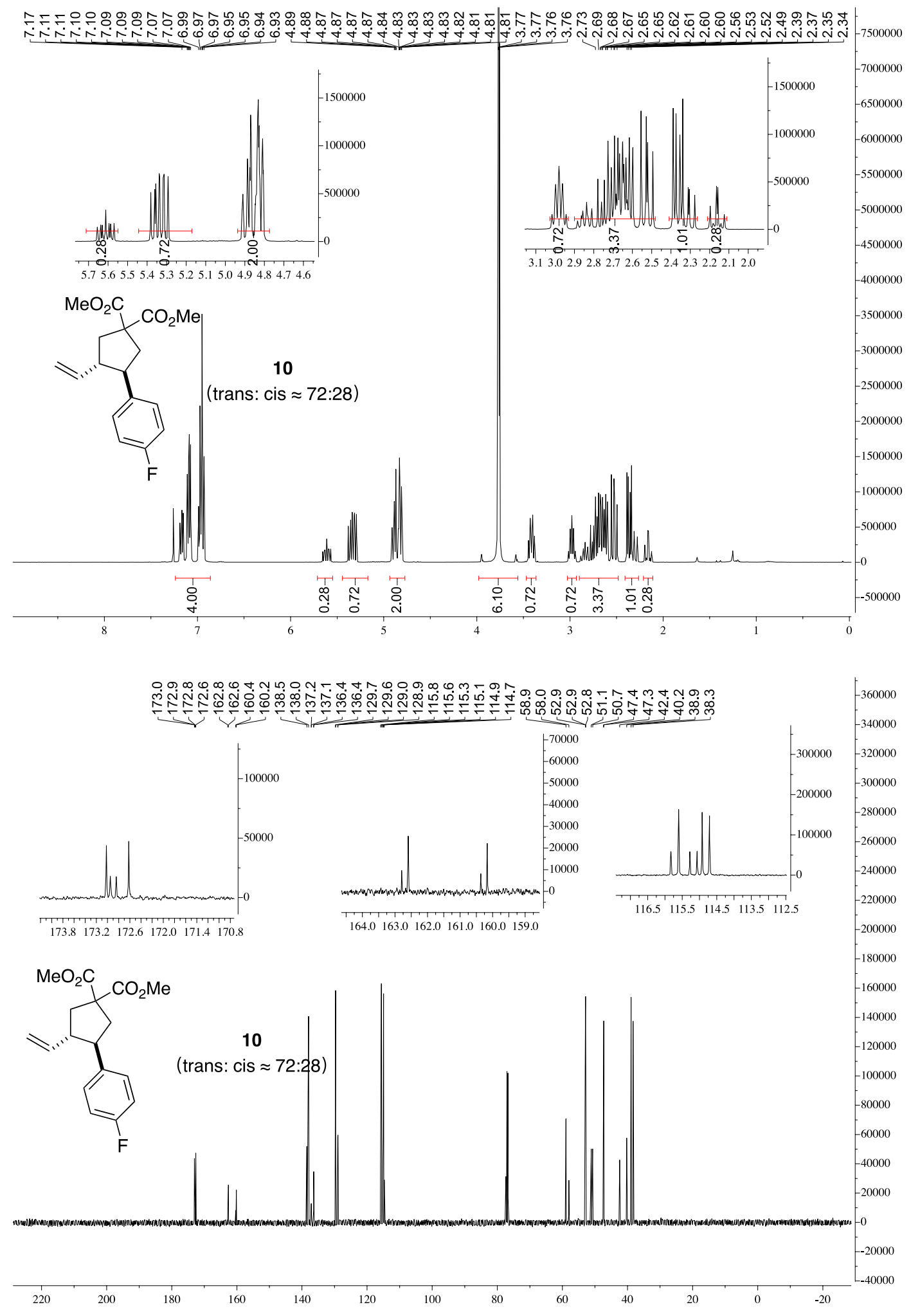




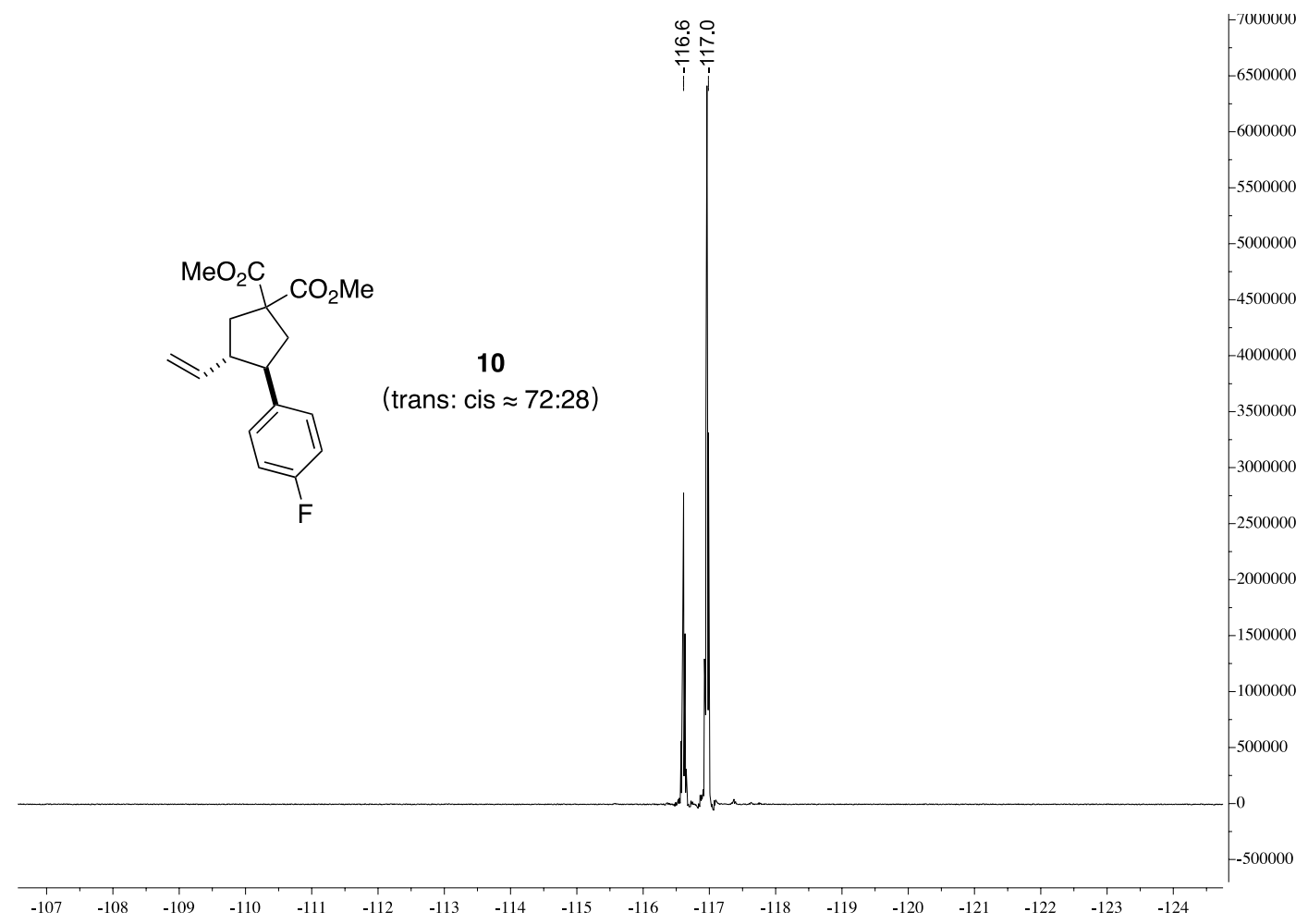



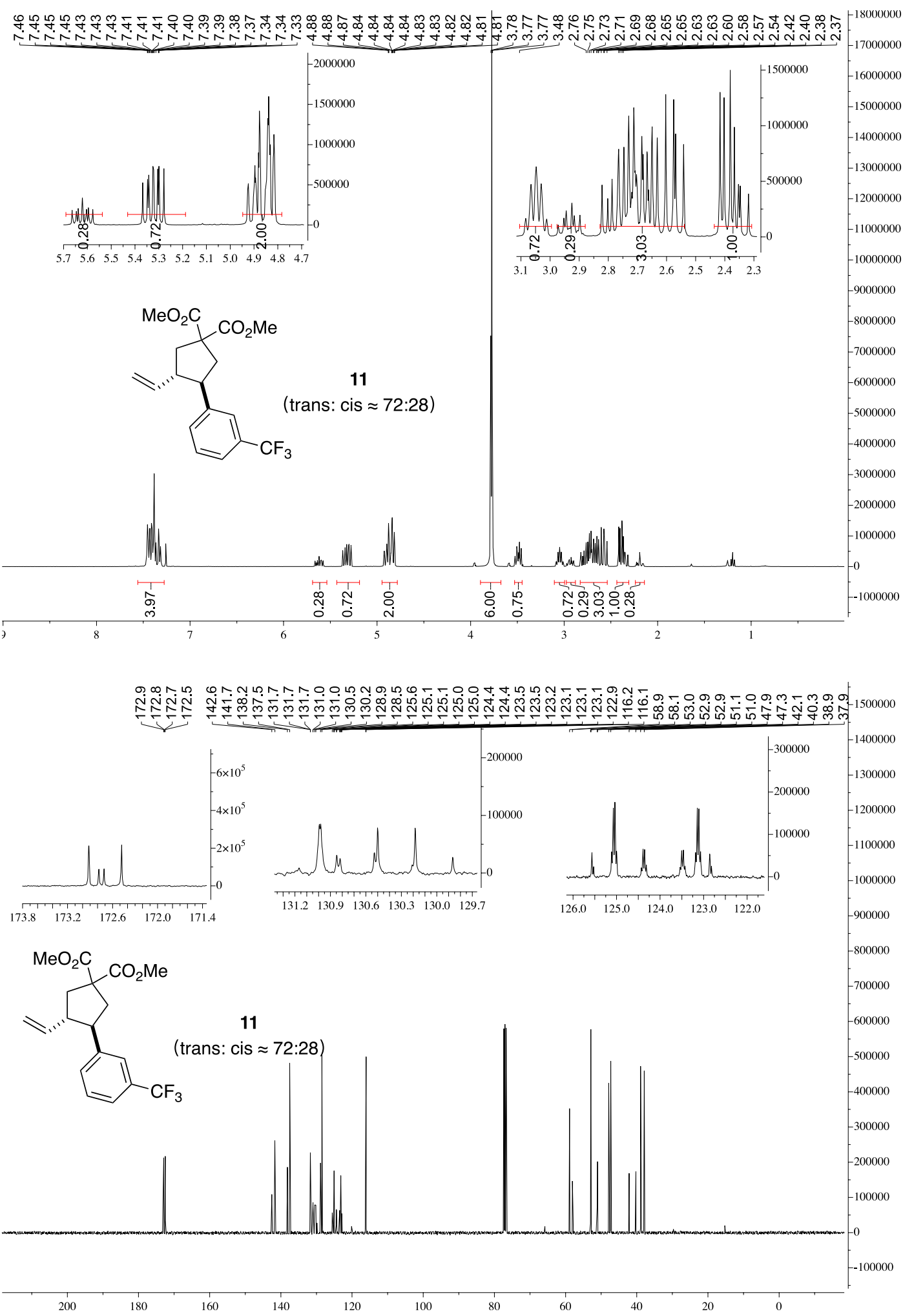


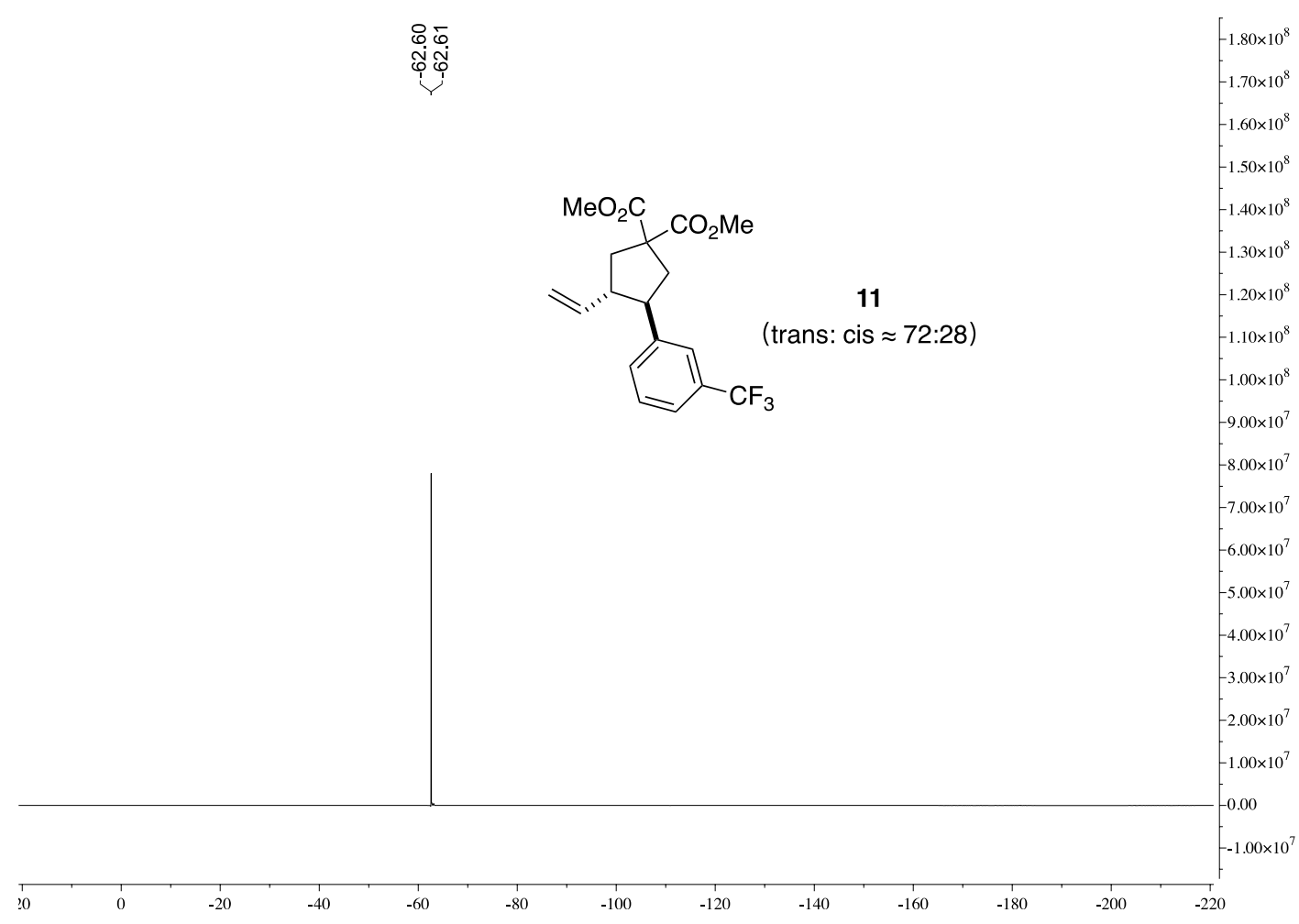




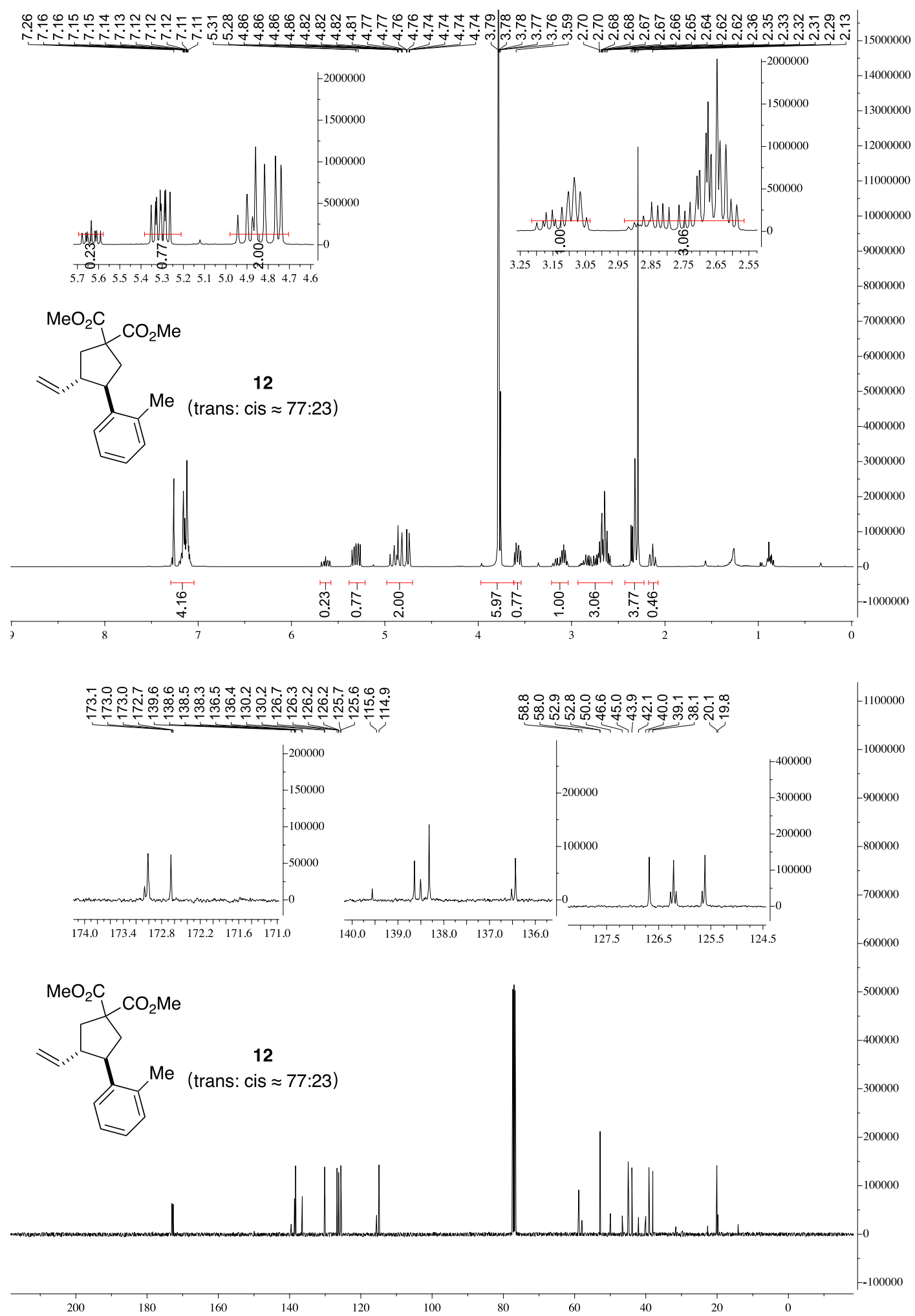




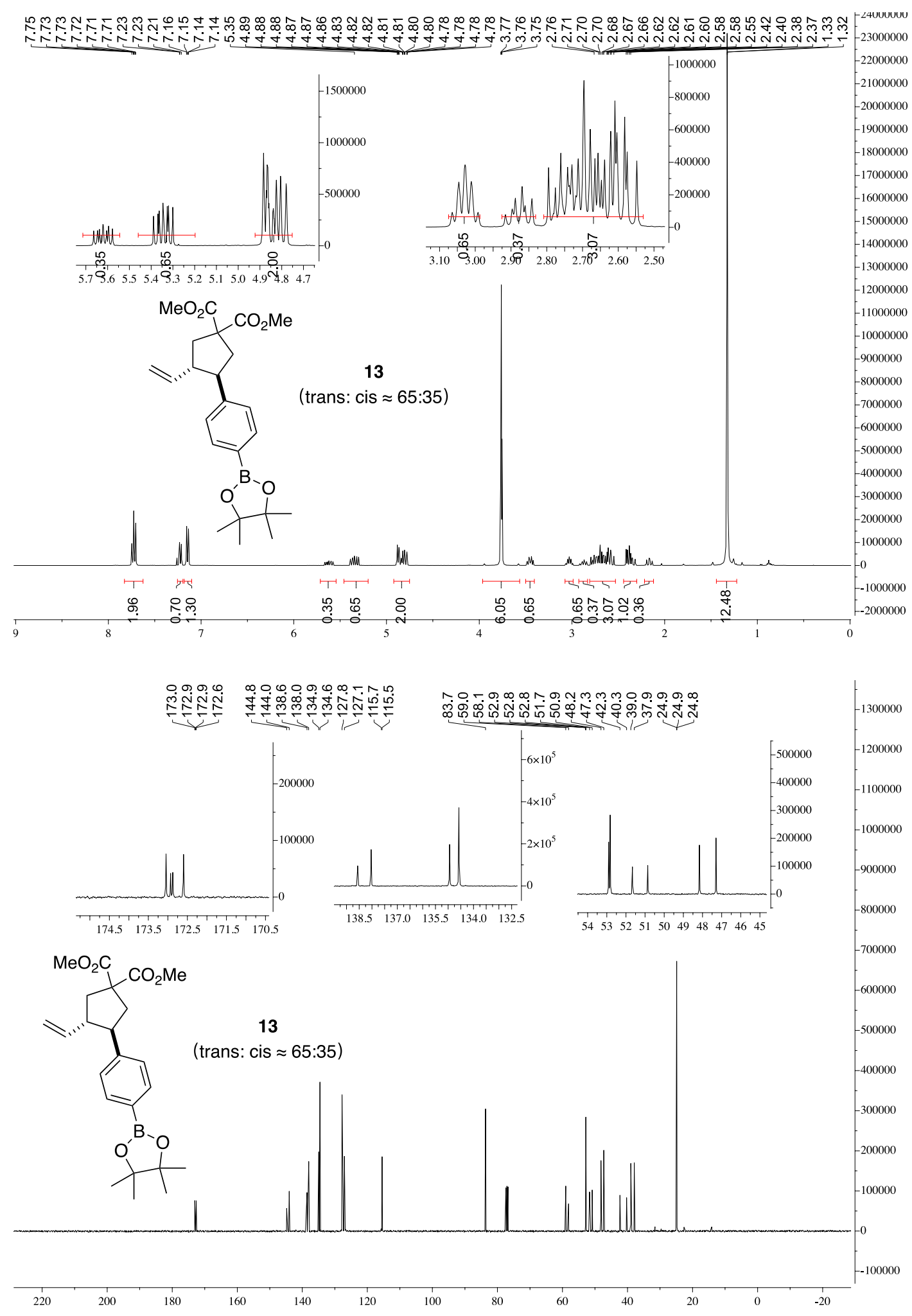




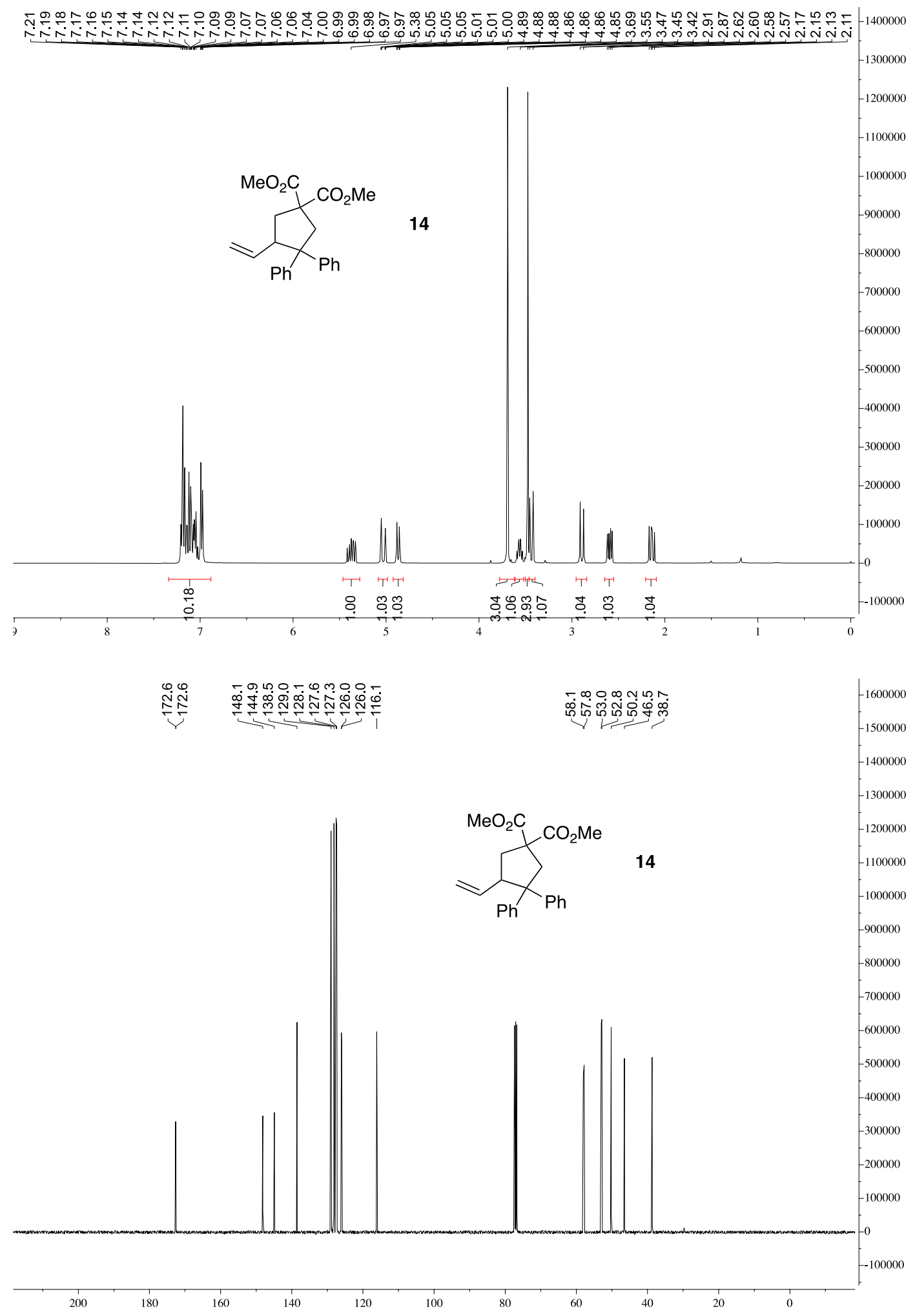




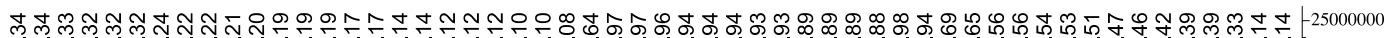

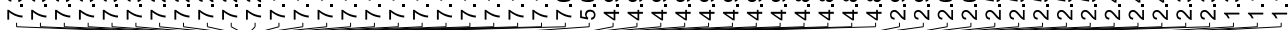
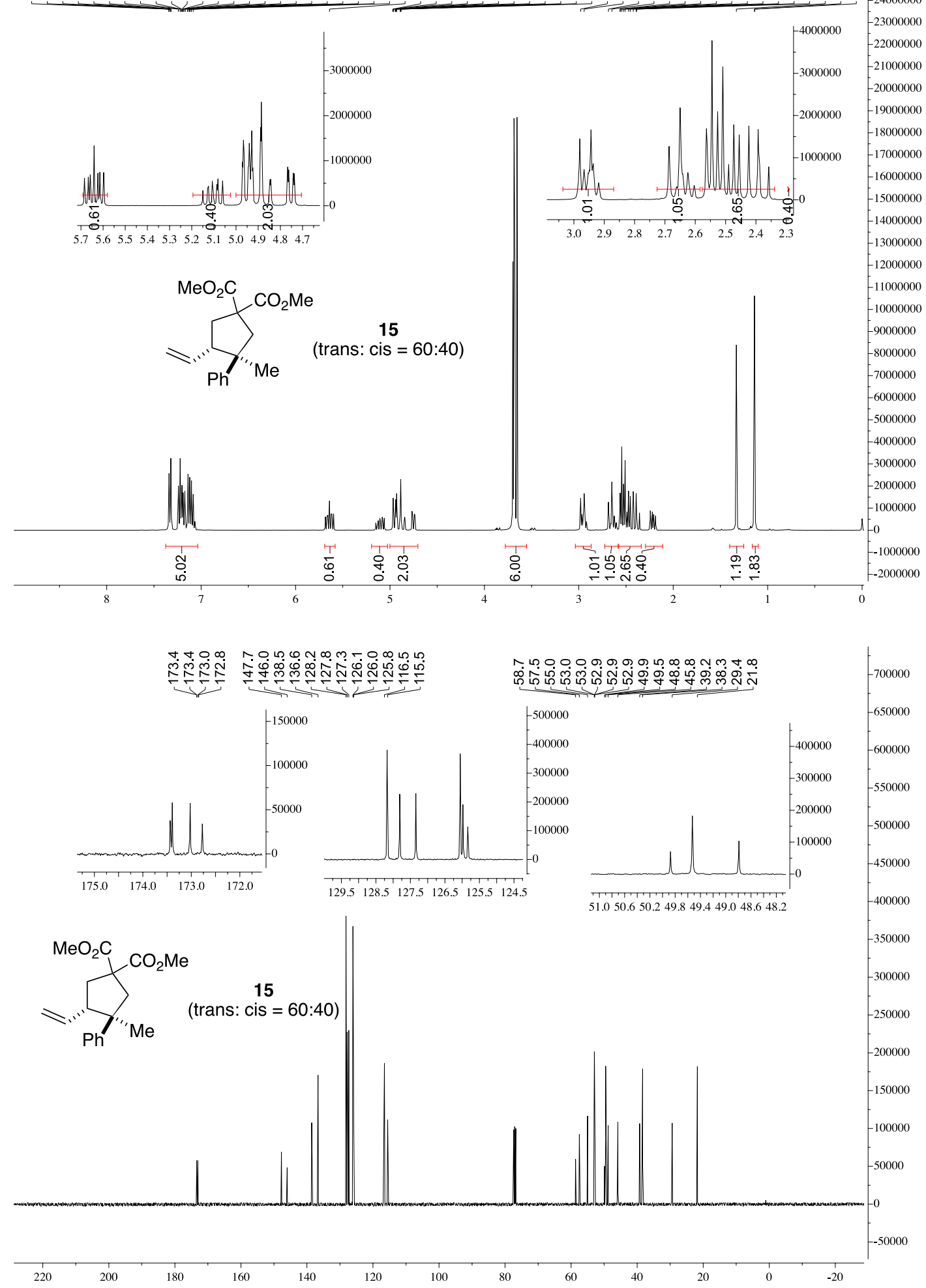


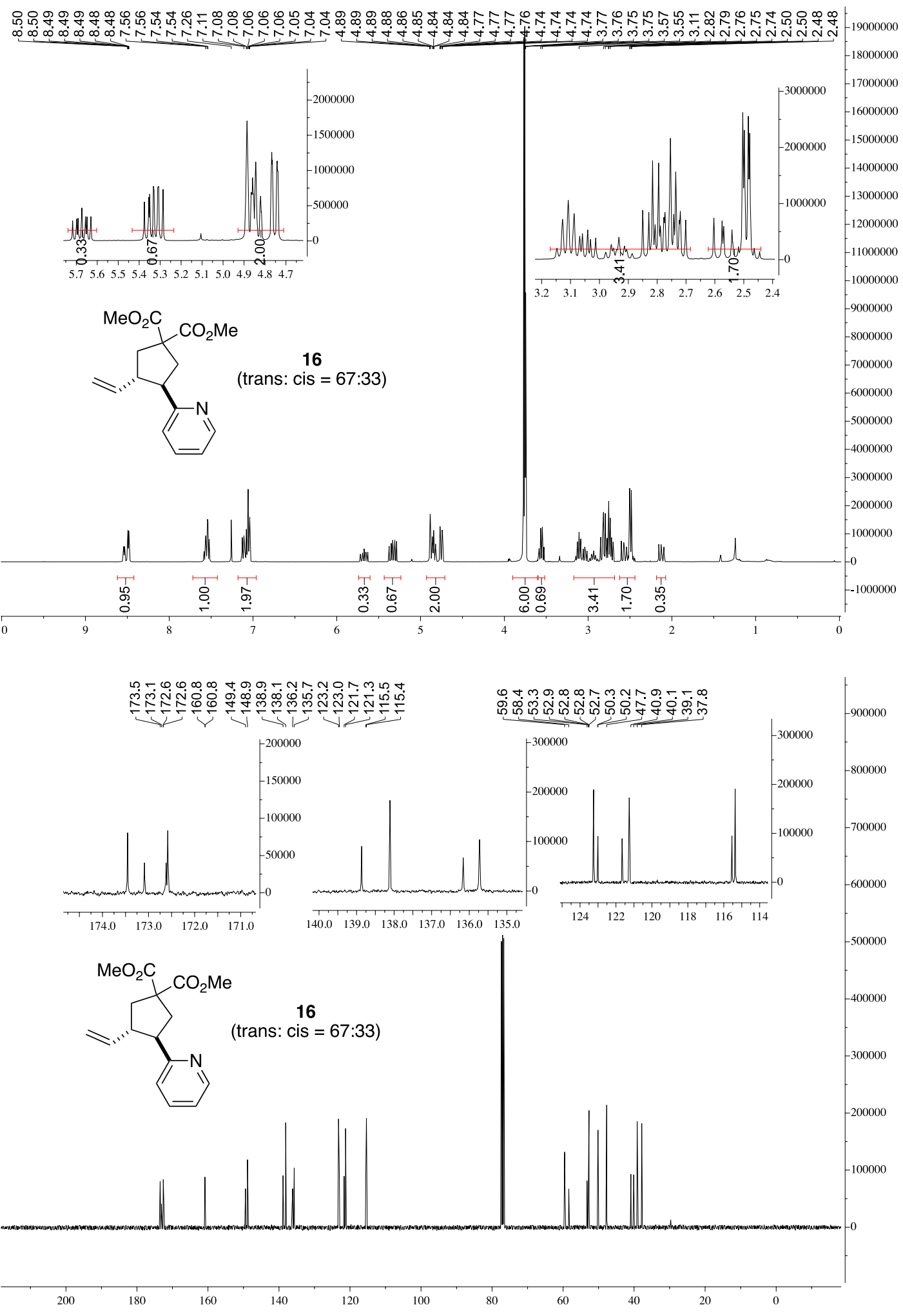




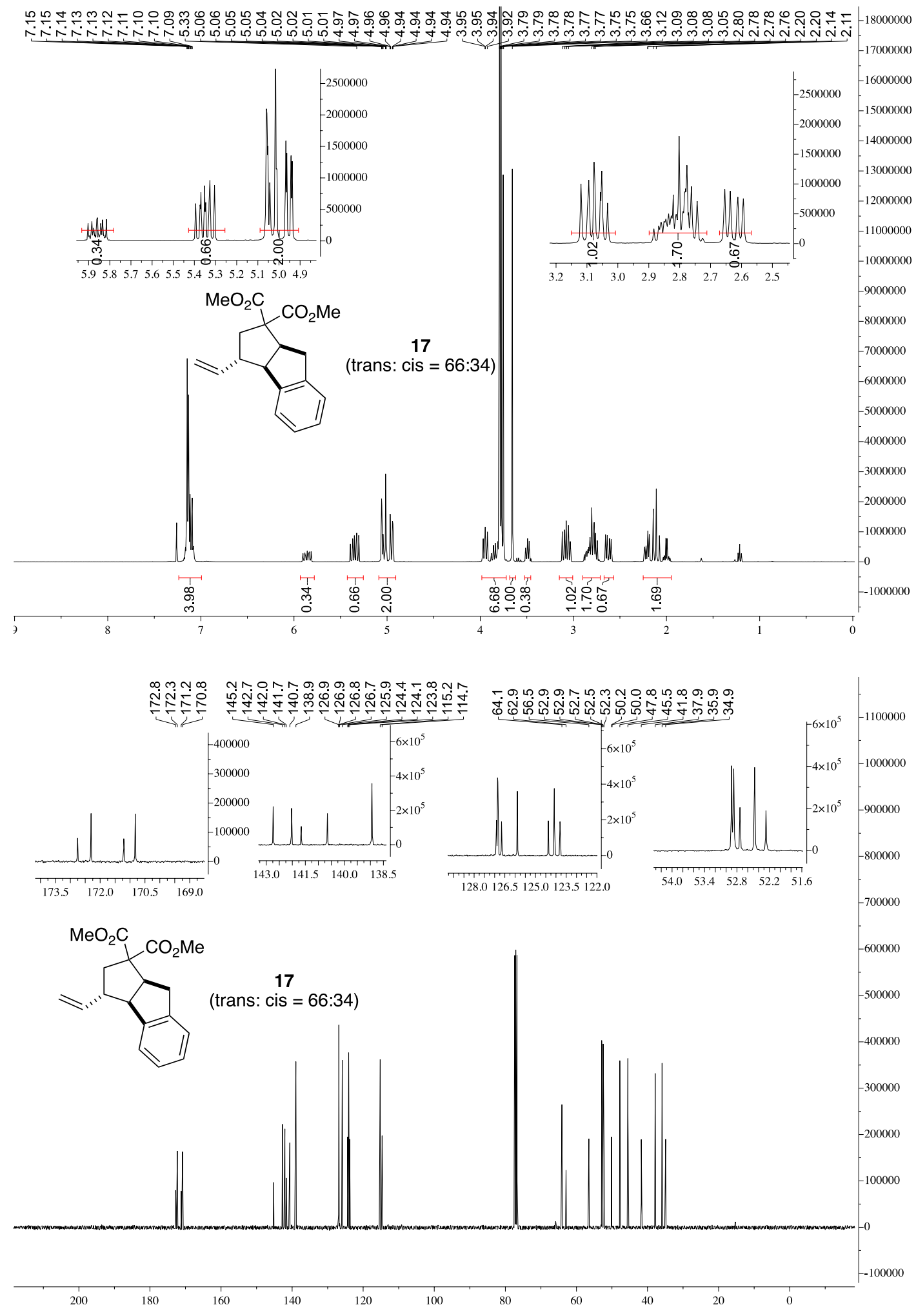




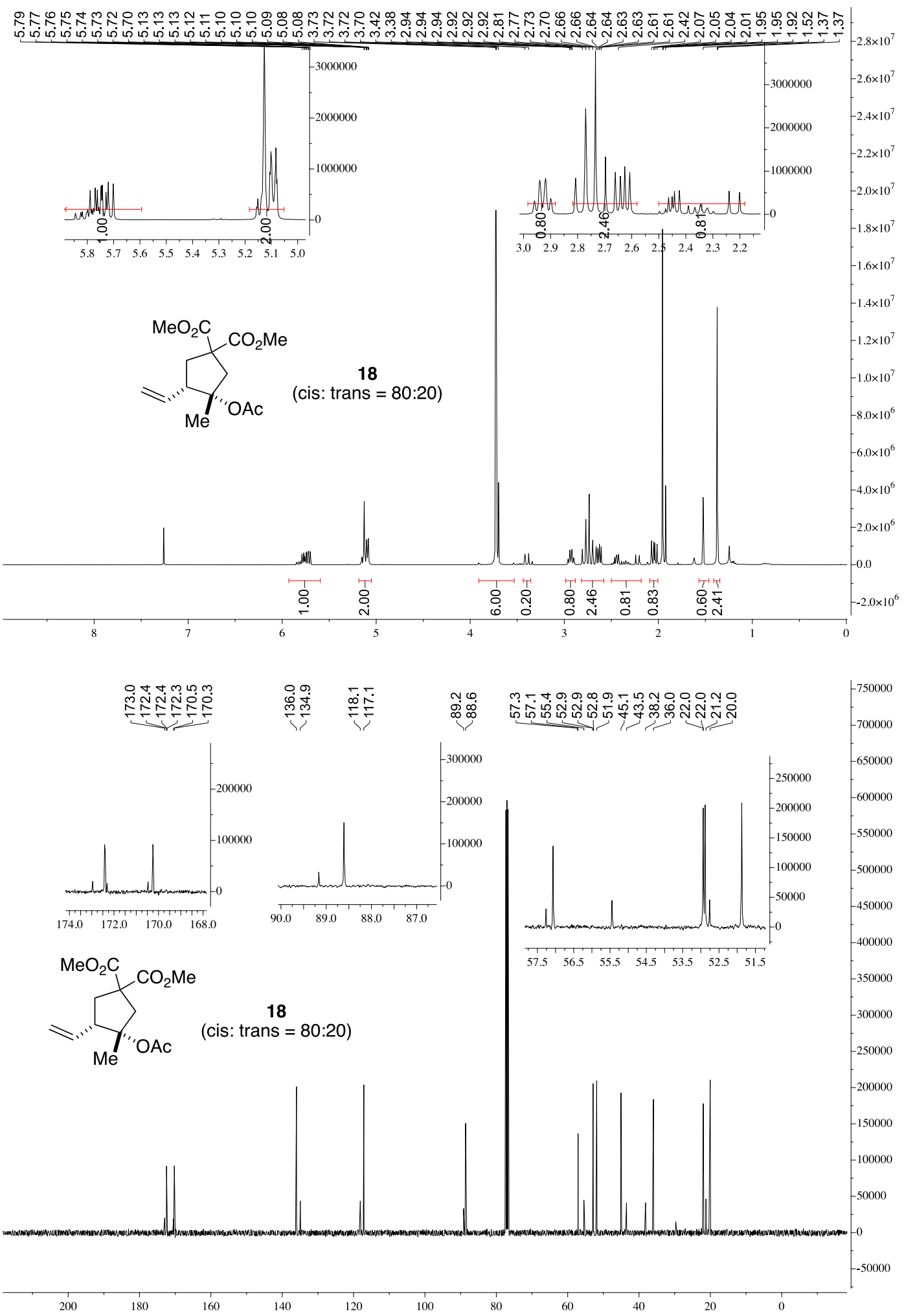




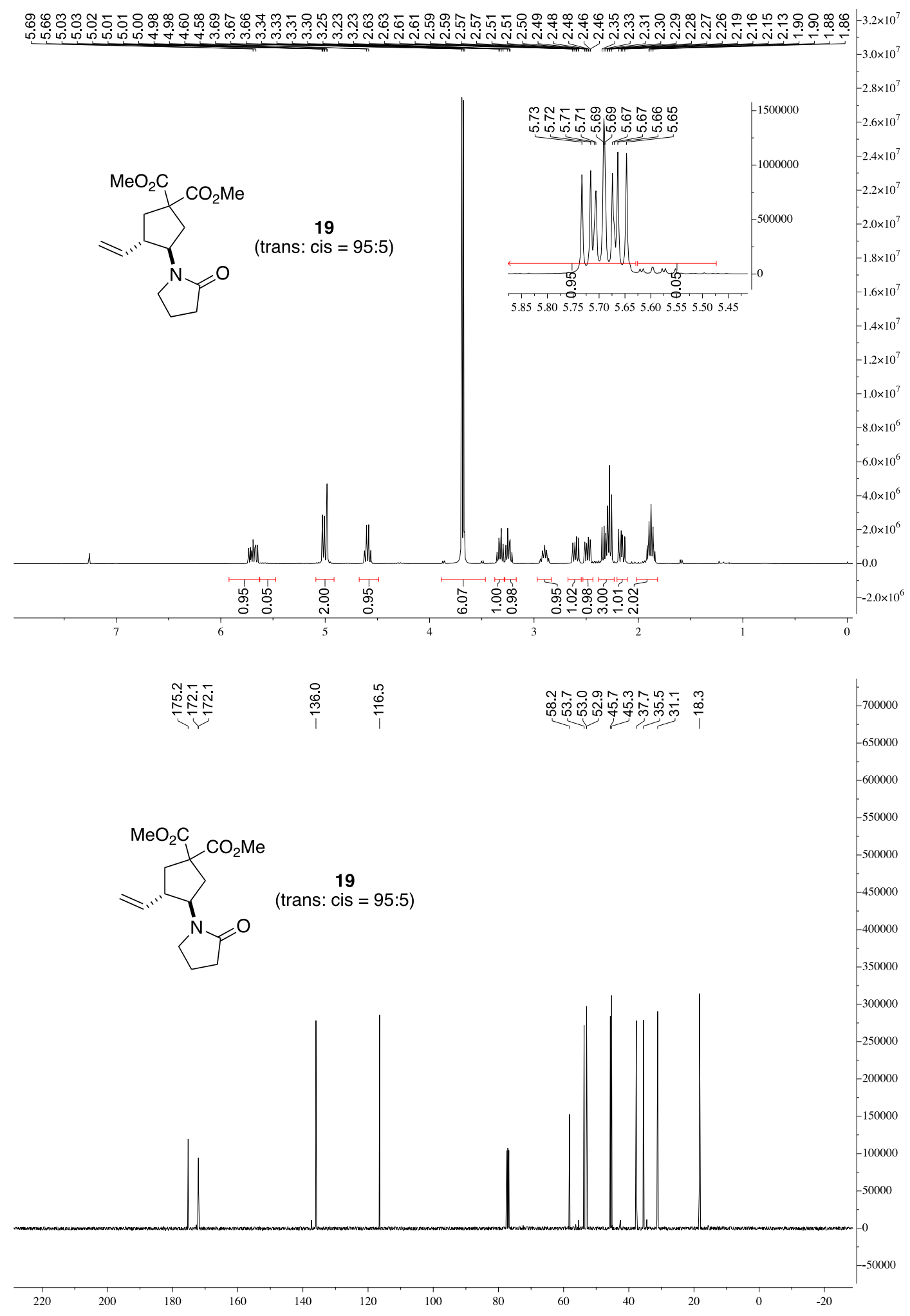




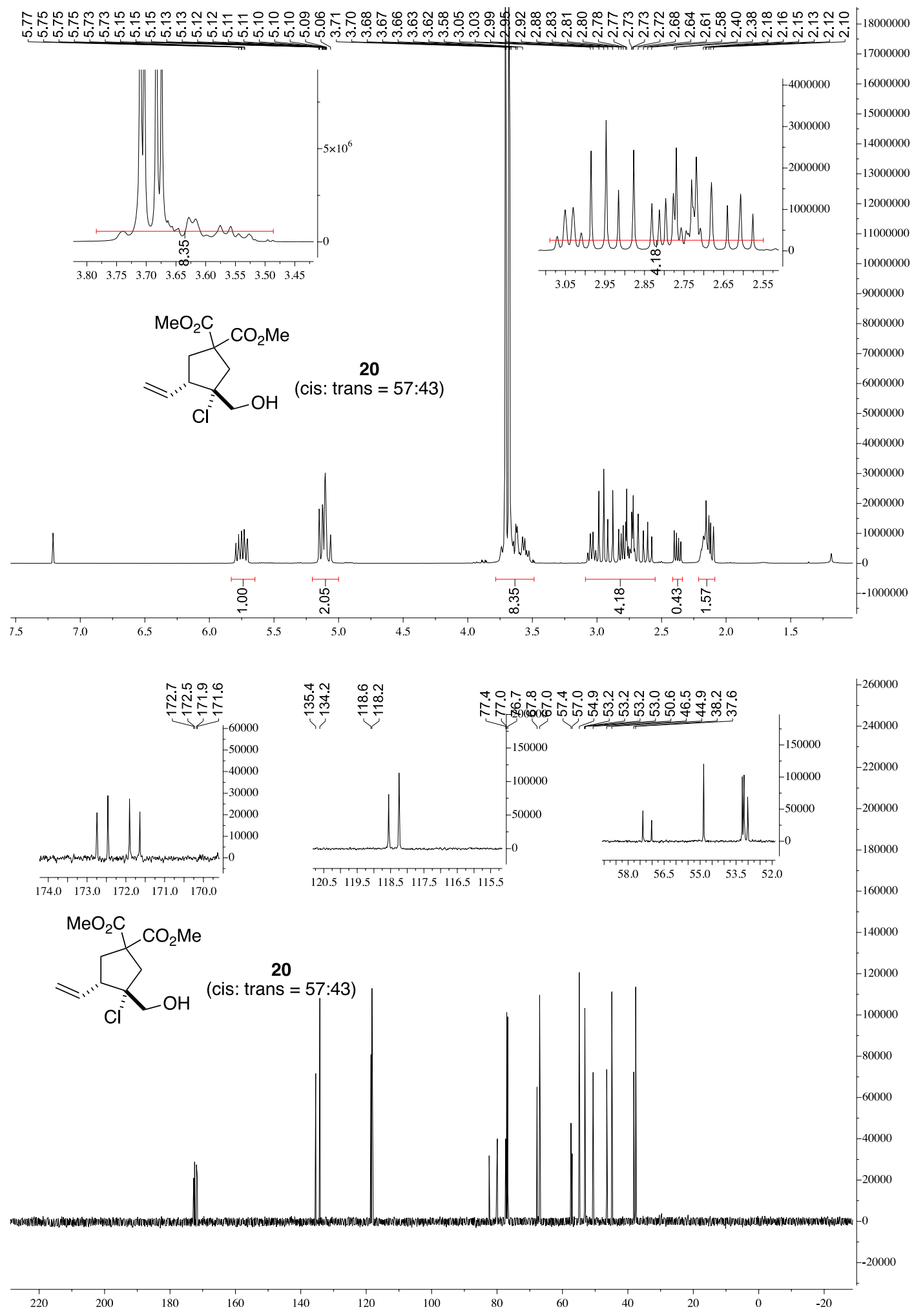



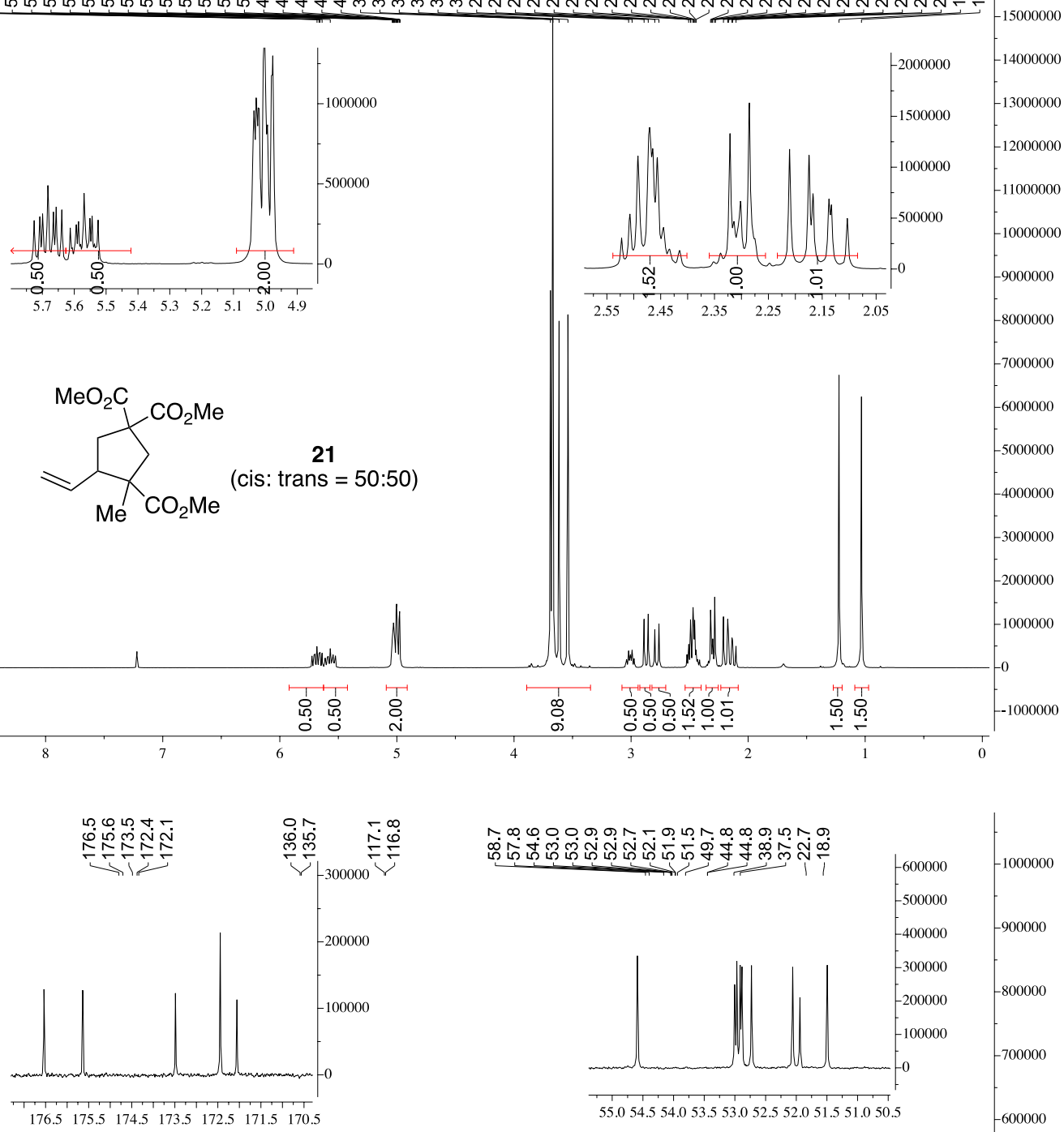

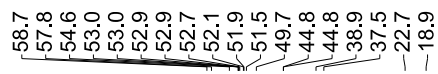

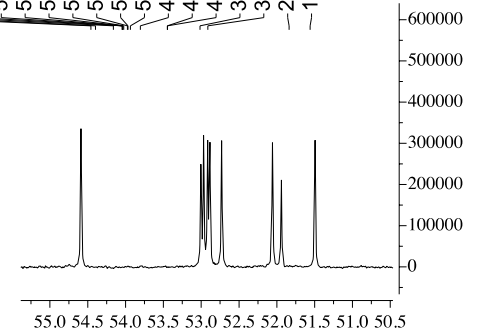

$-1000000$
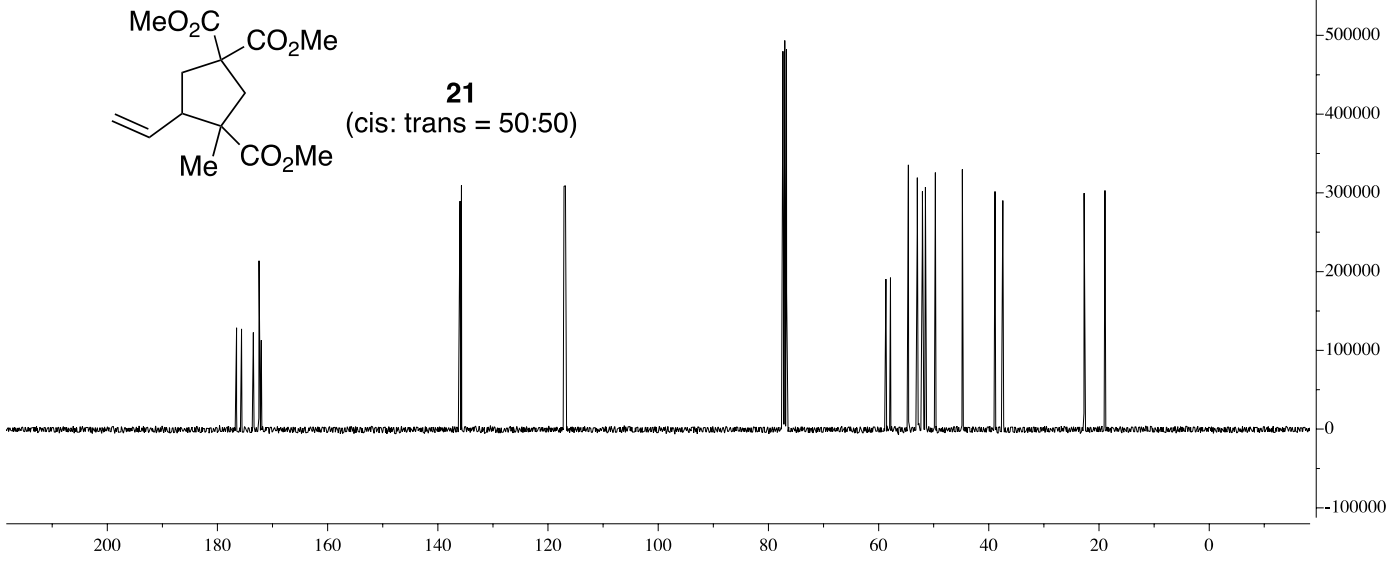


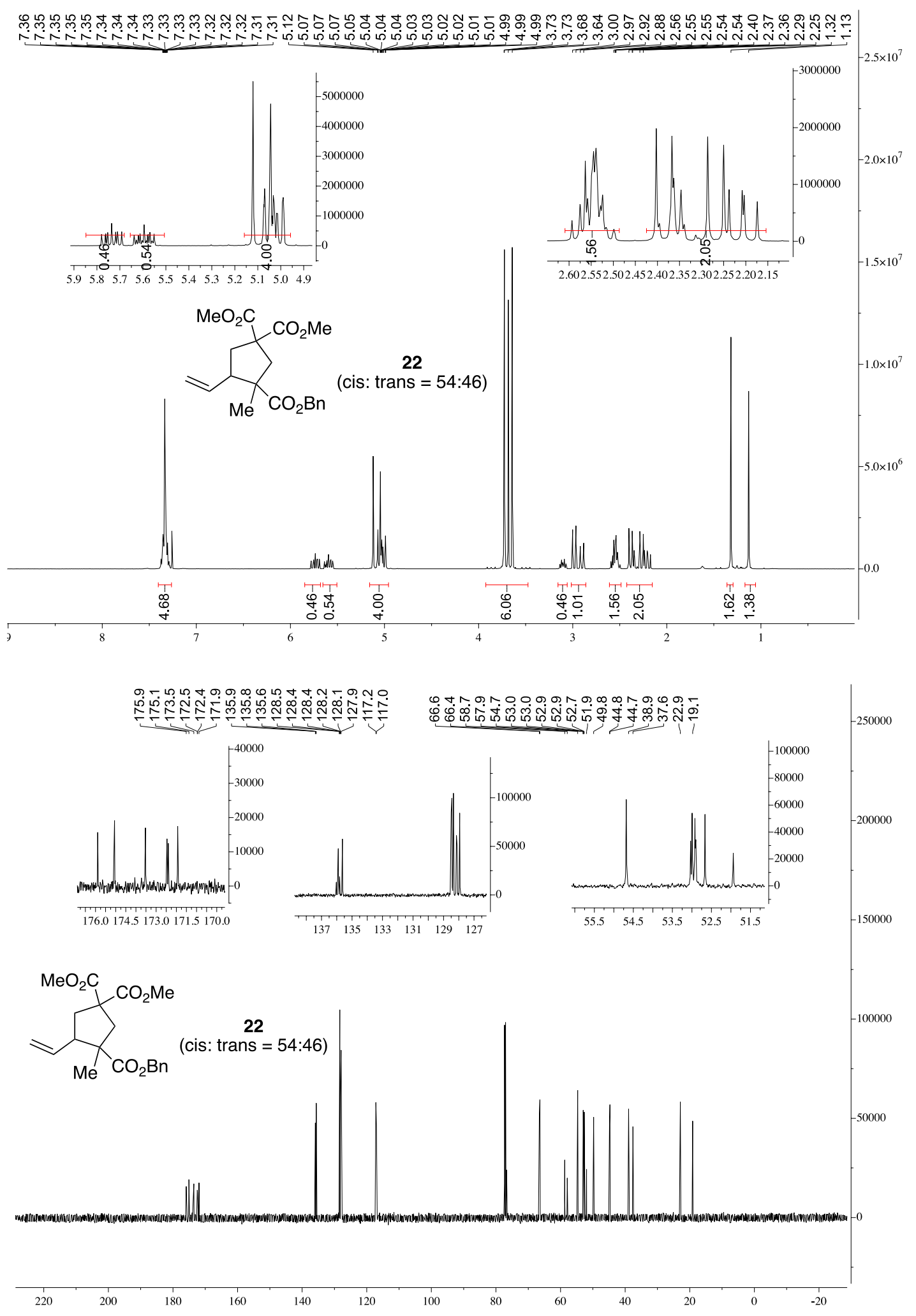




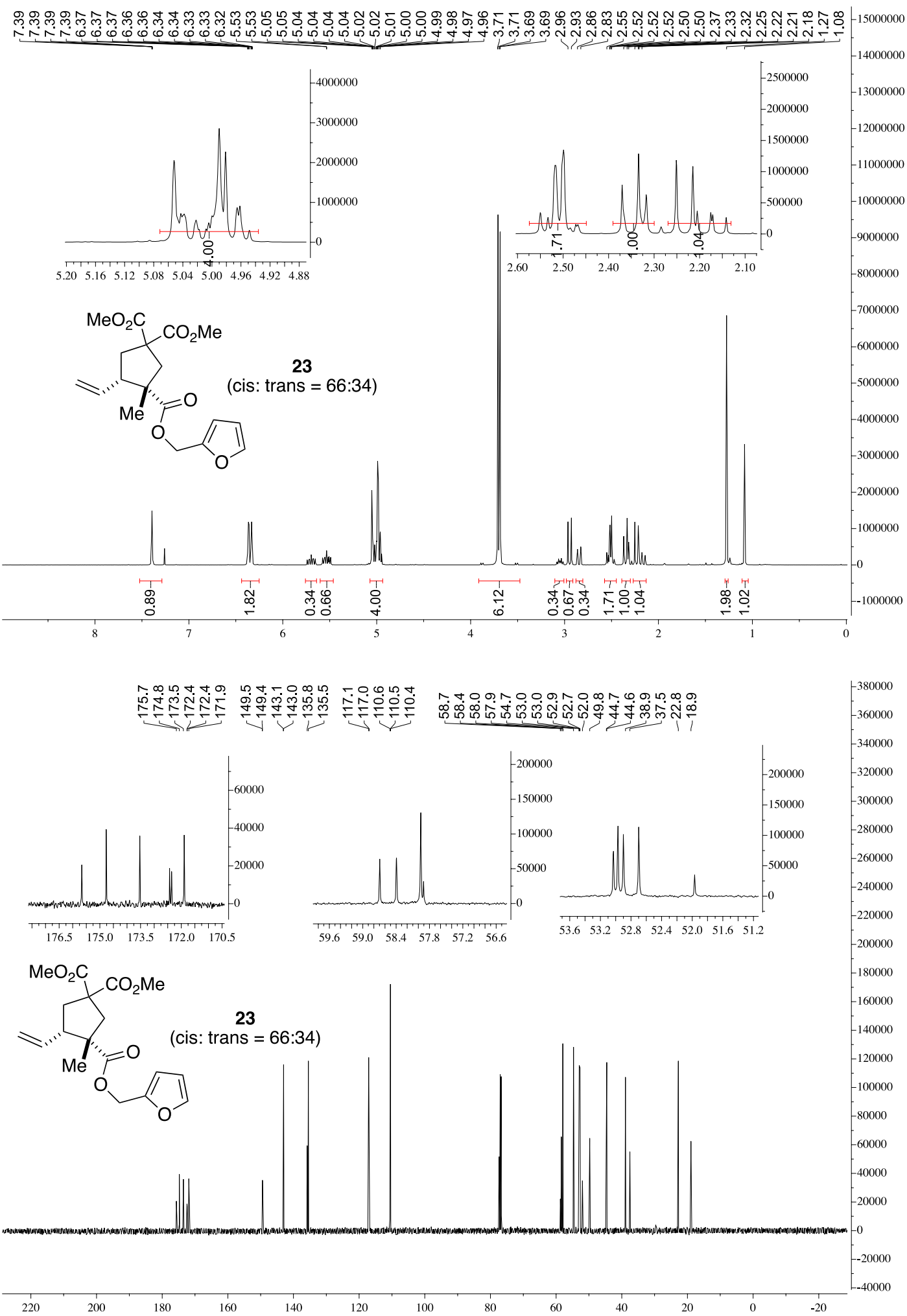




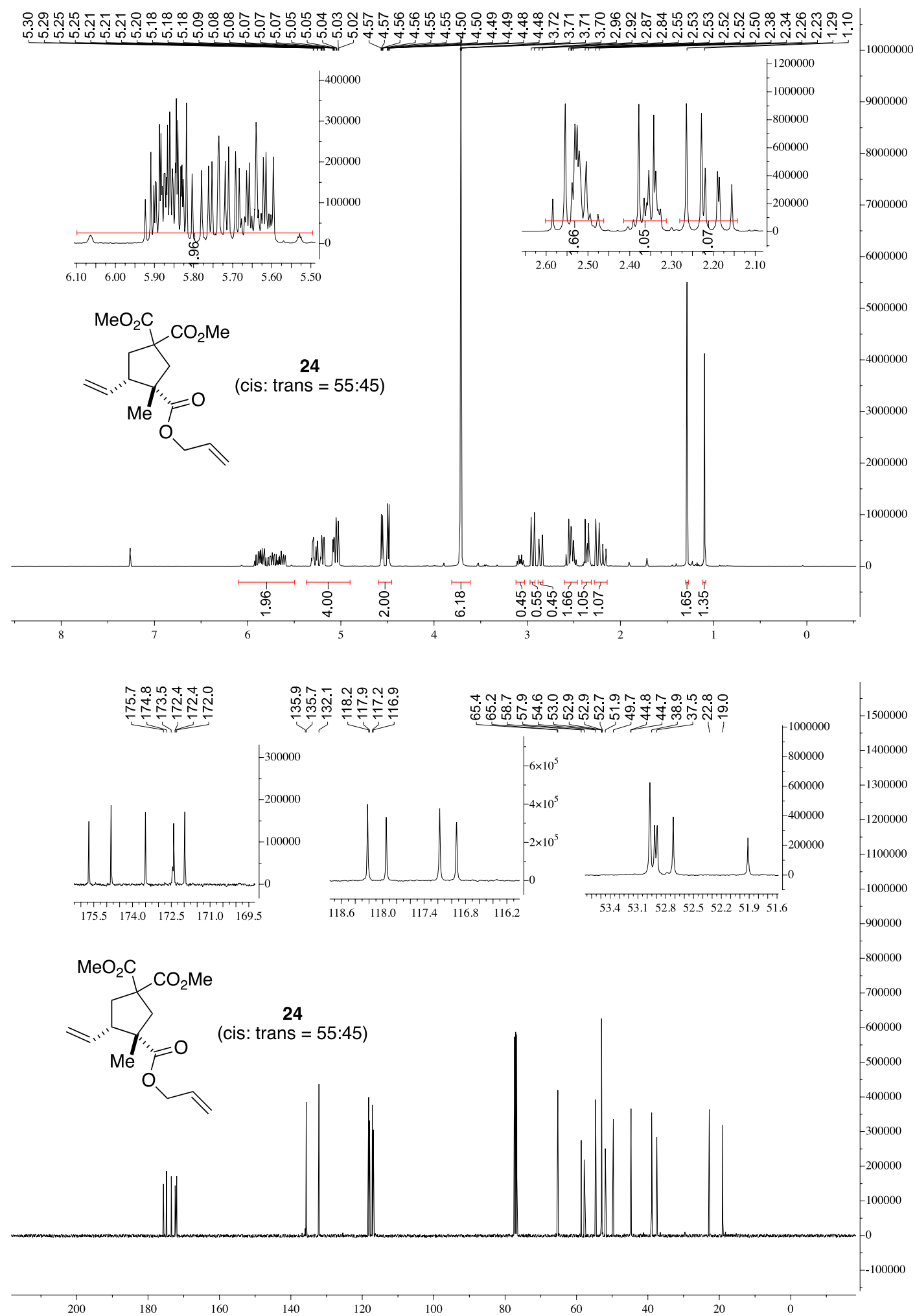




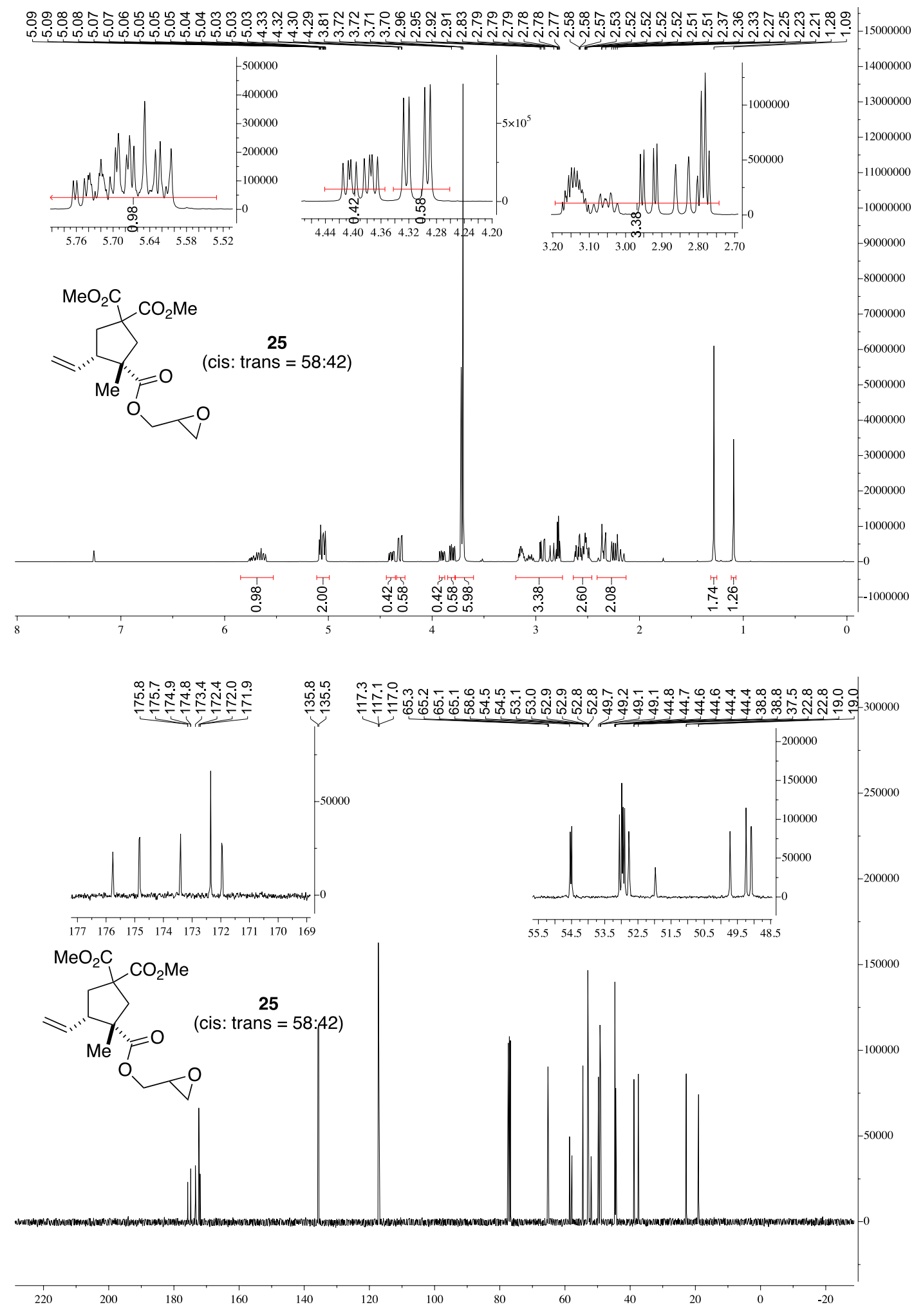



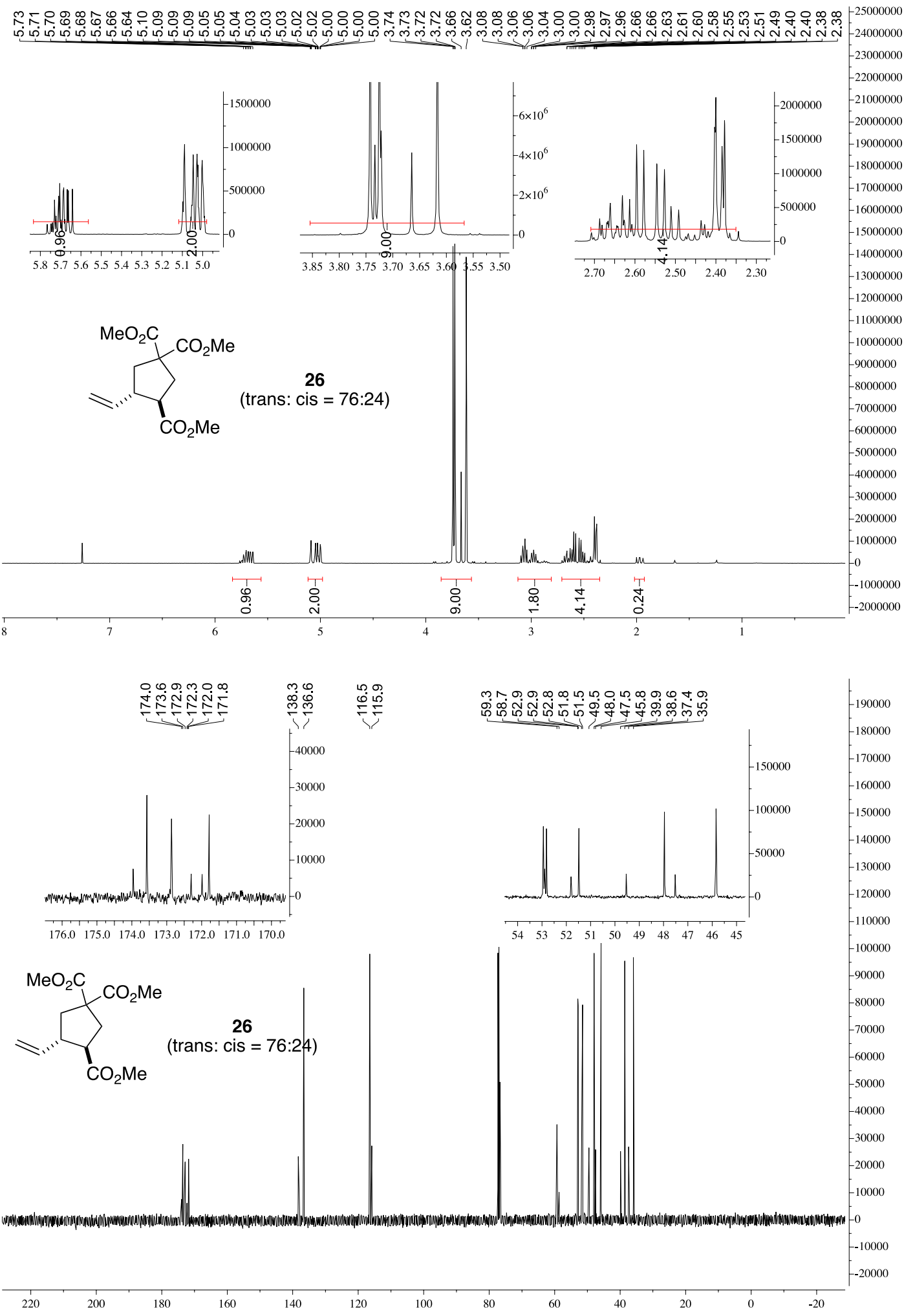

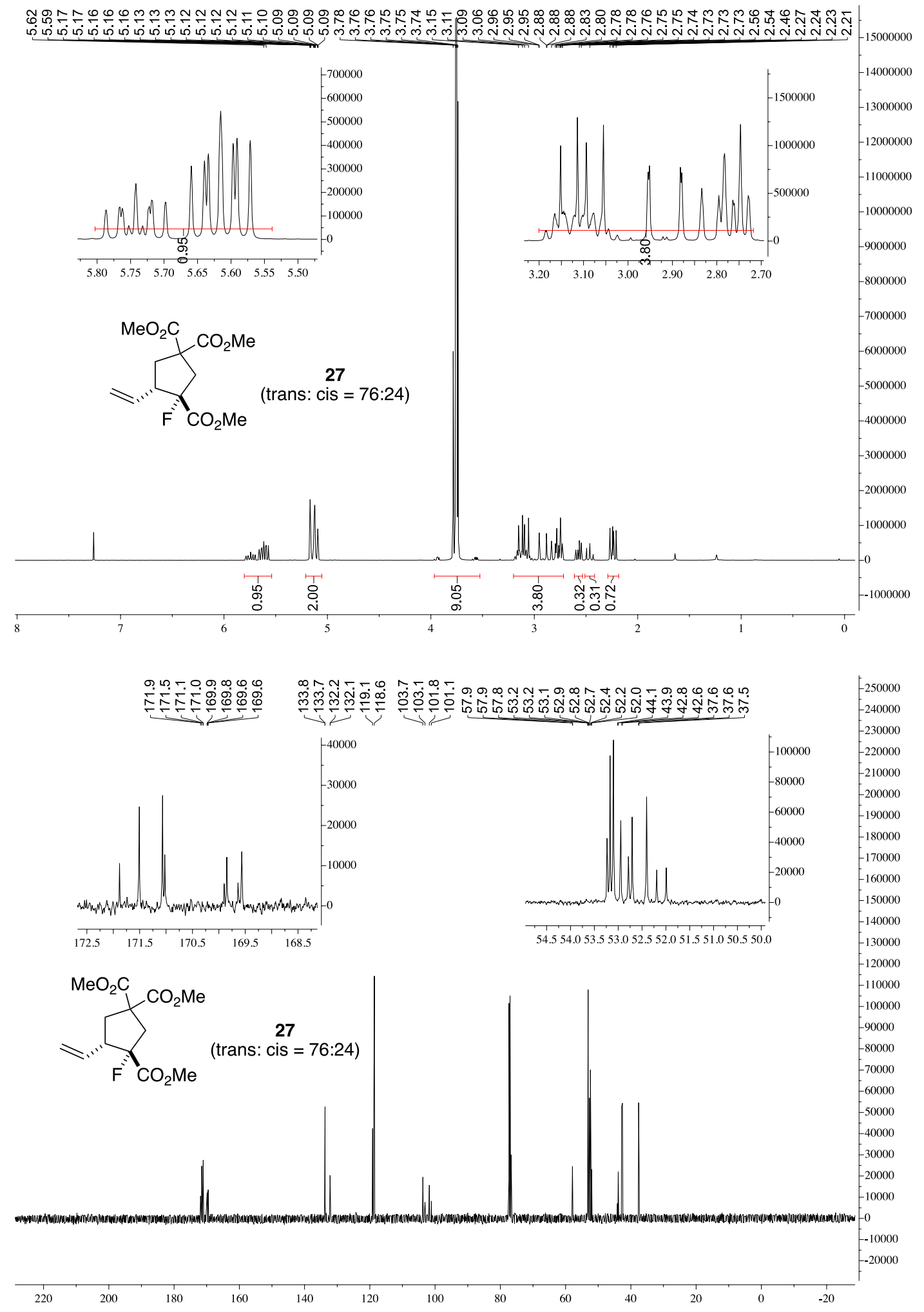


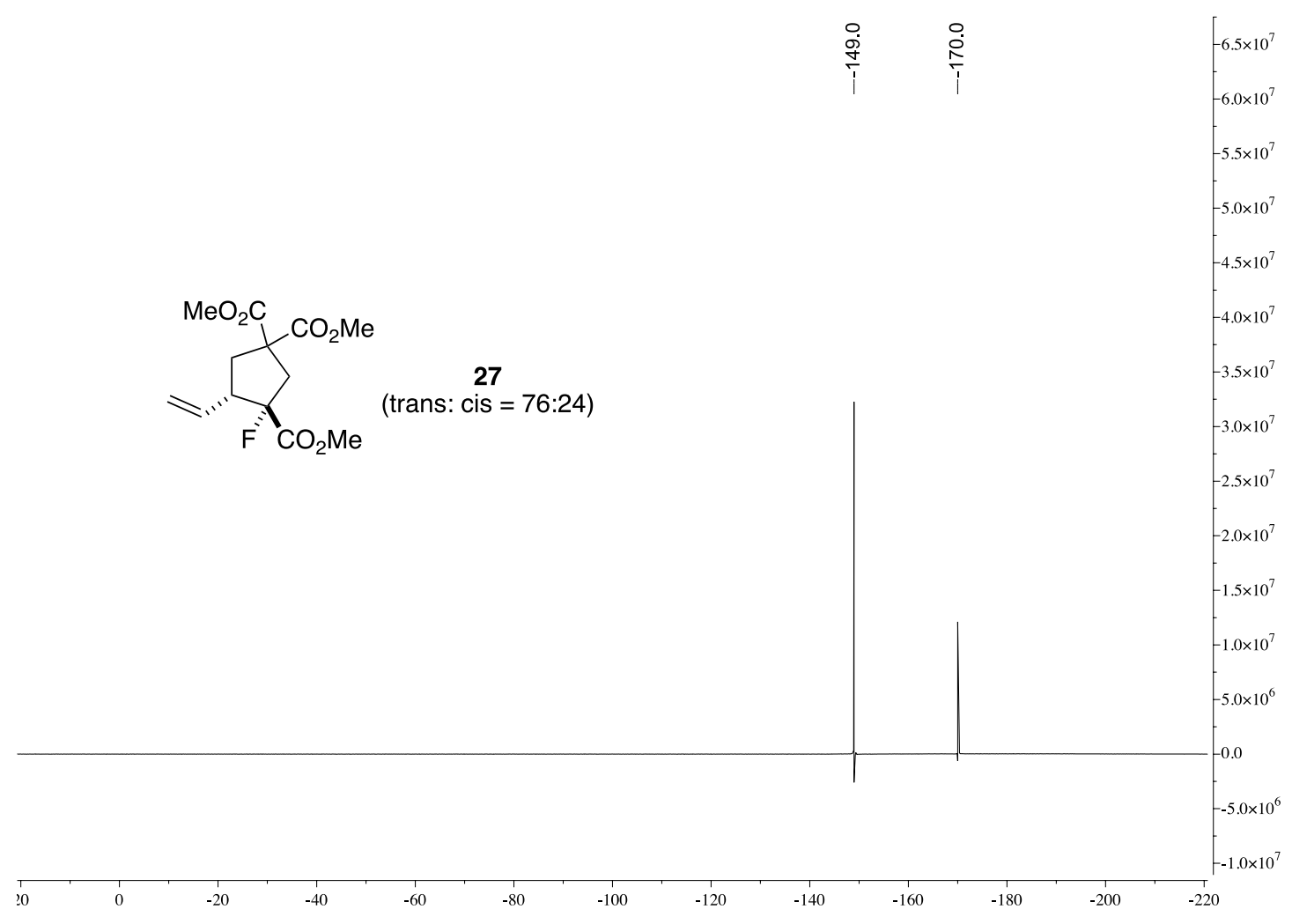




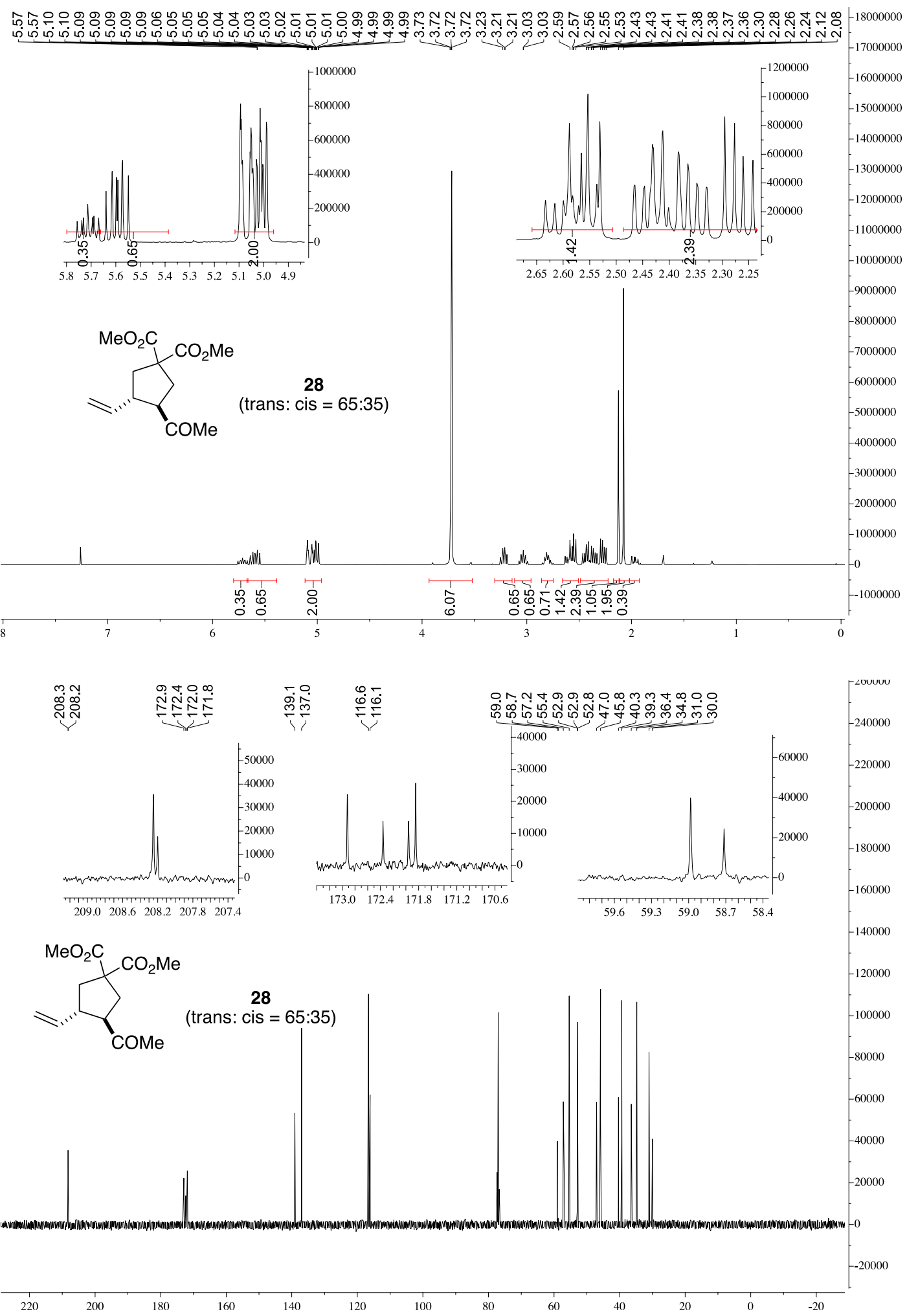




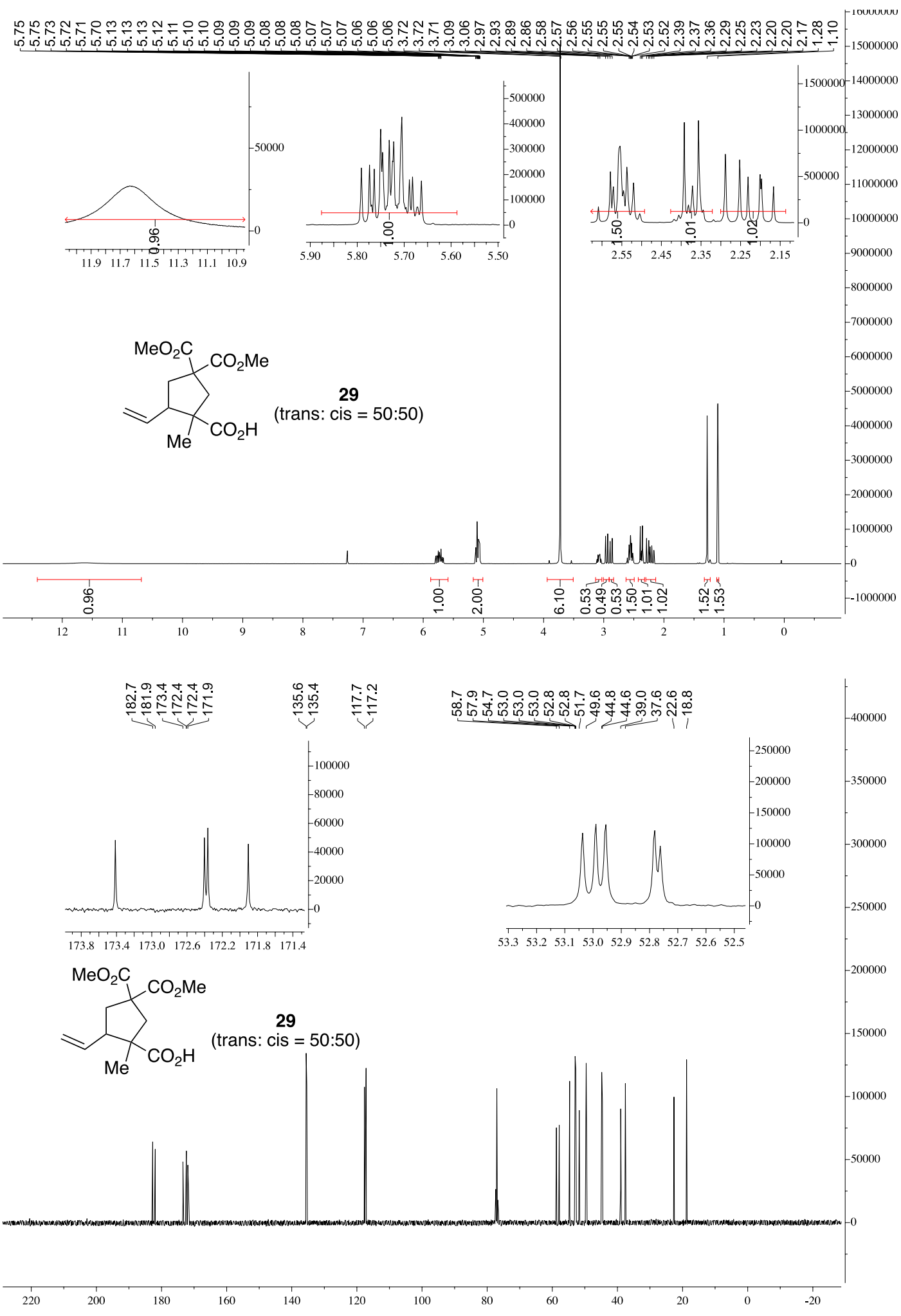




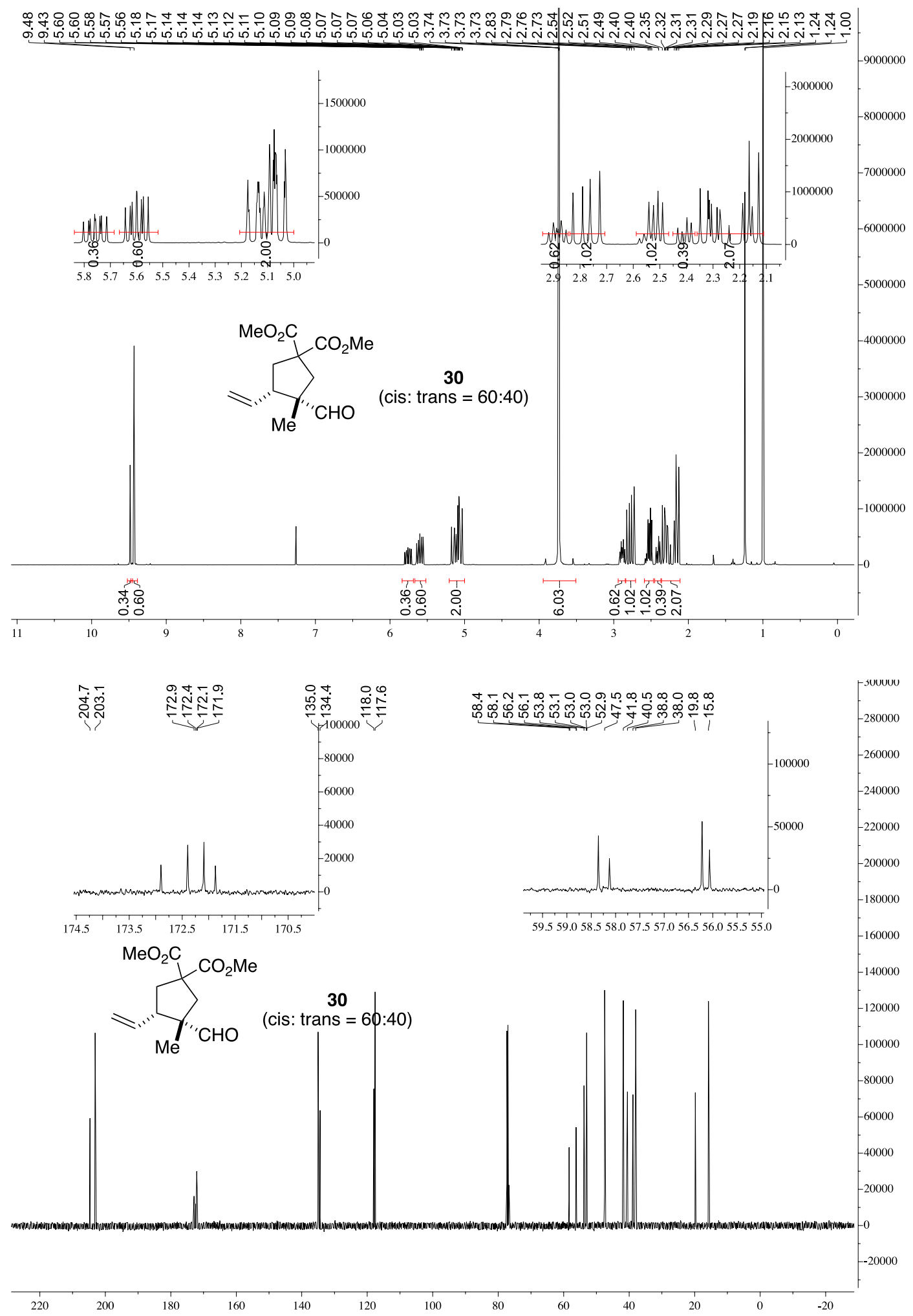




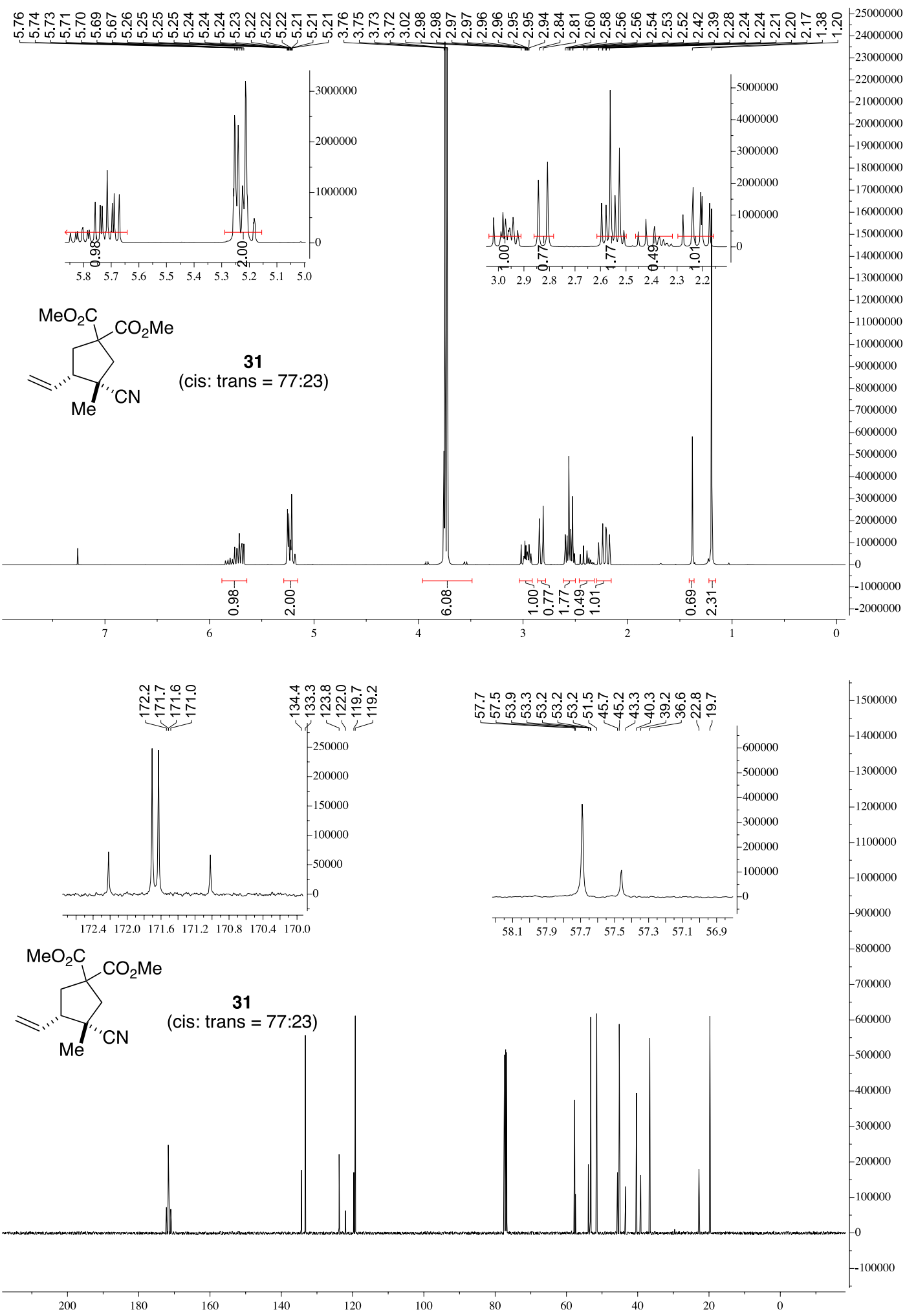




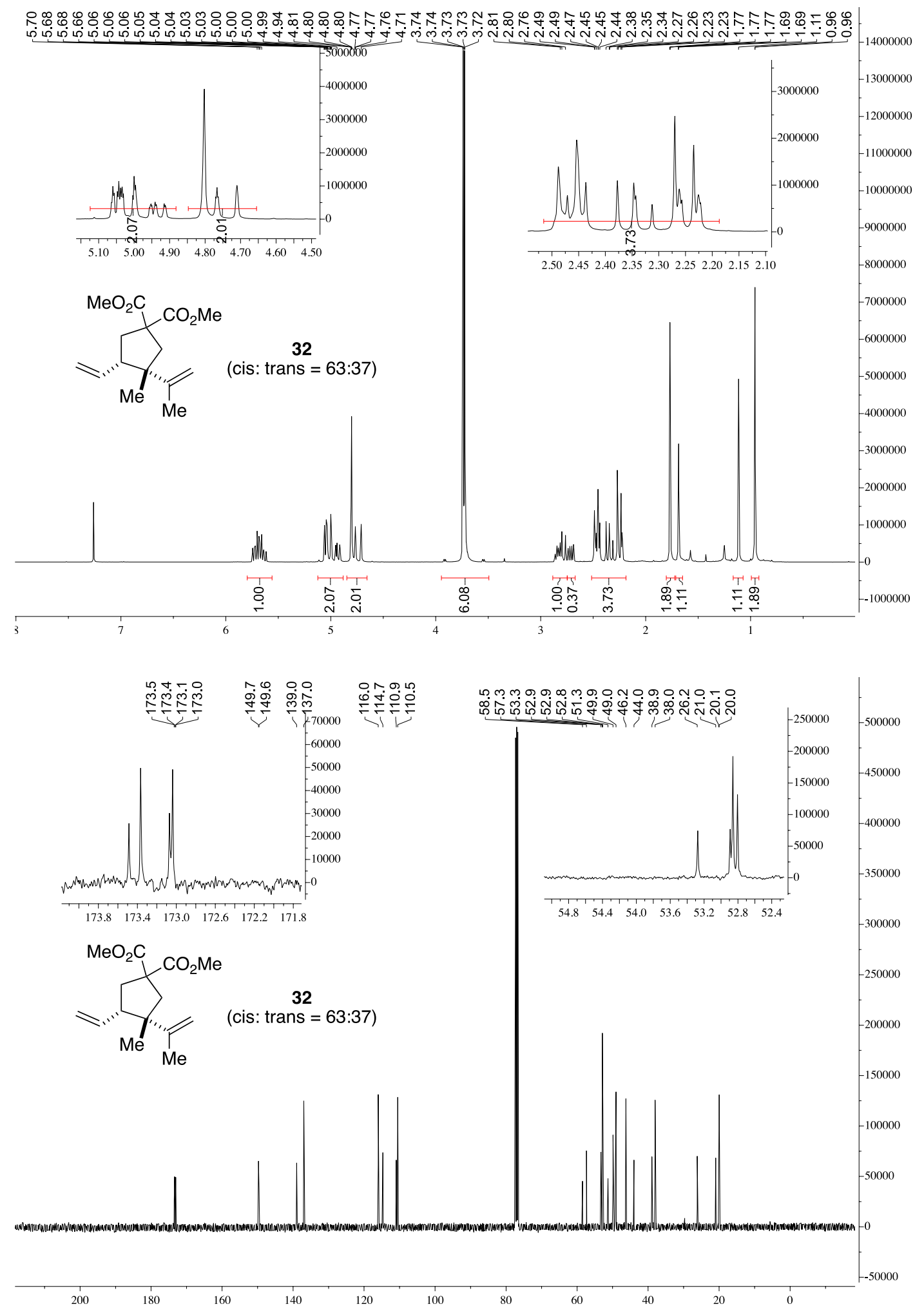




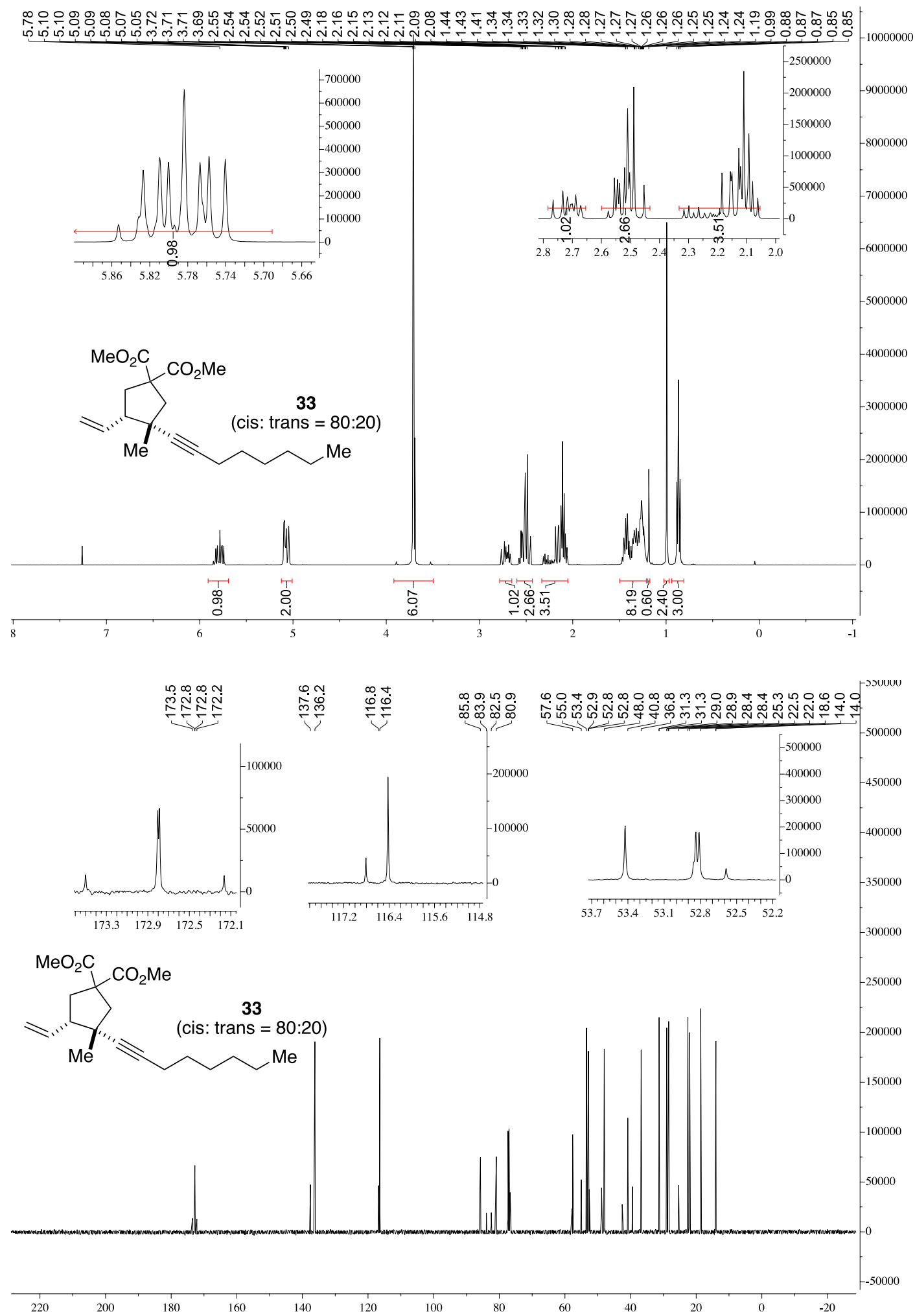




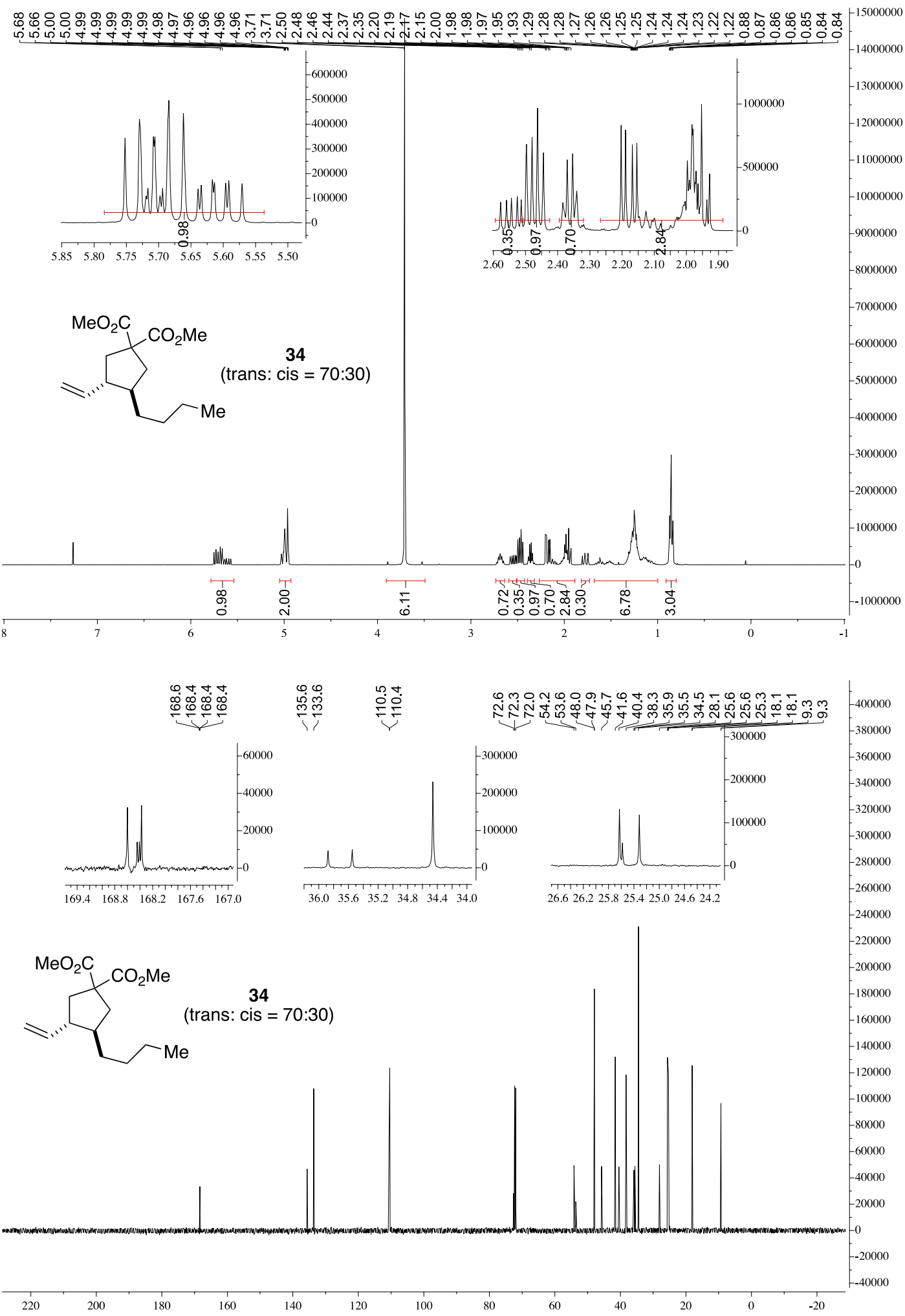



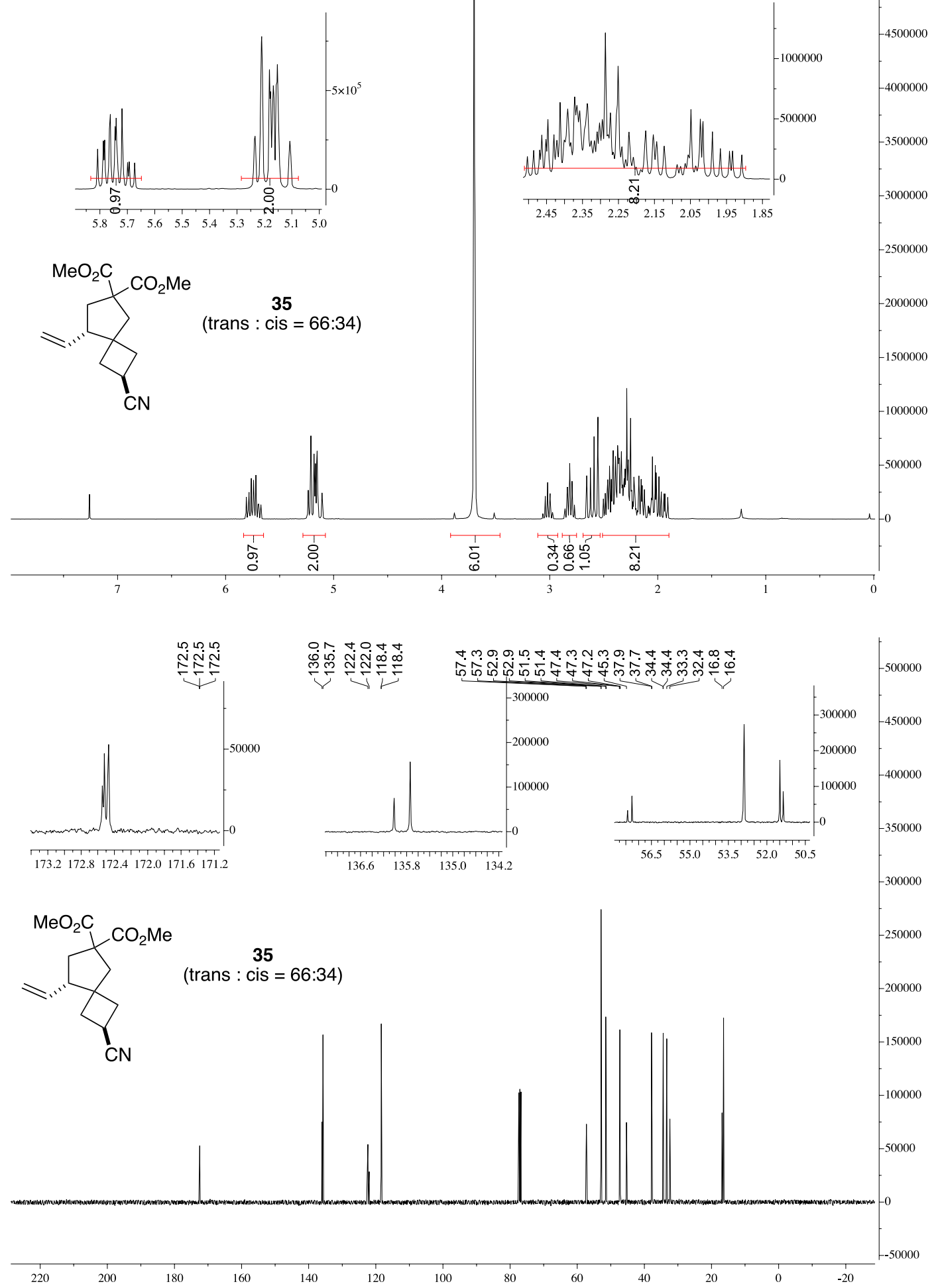


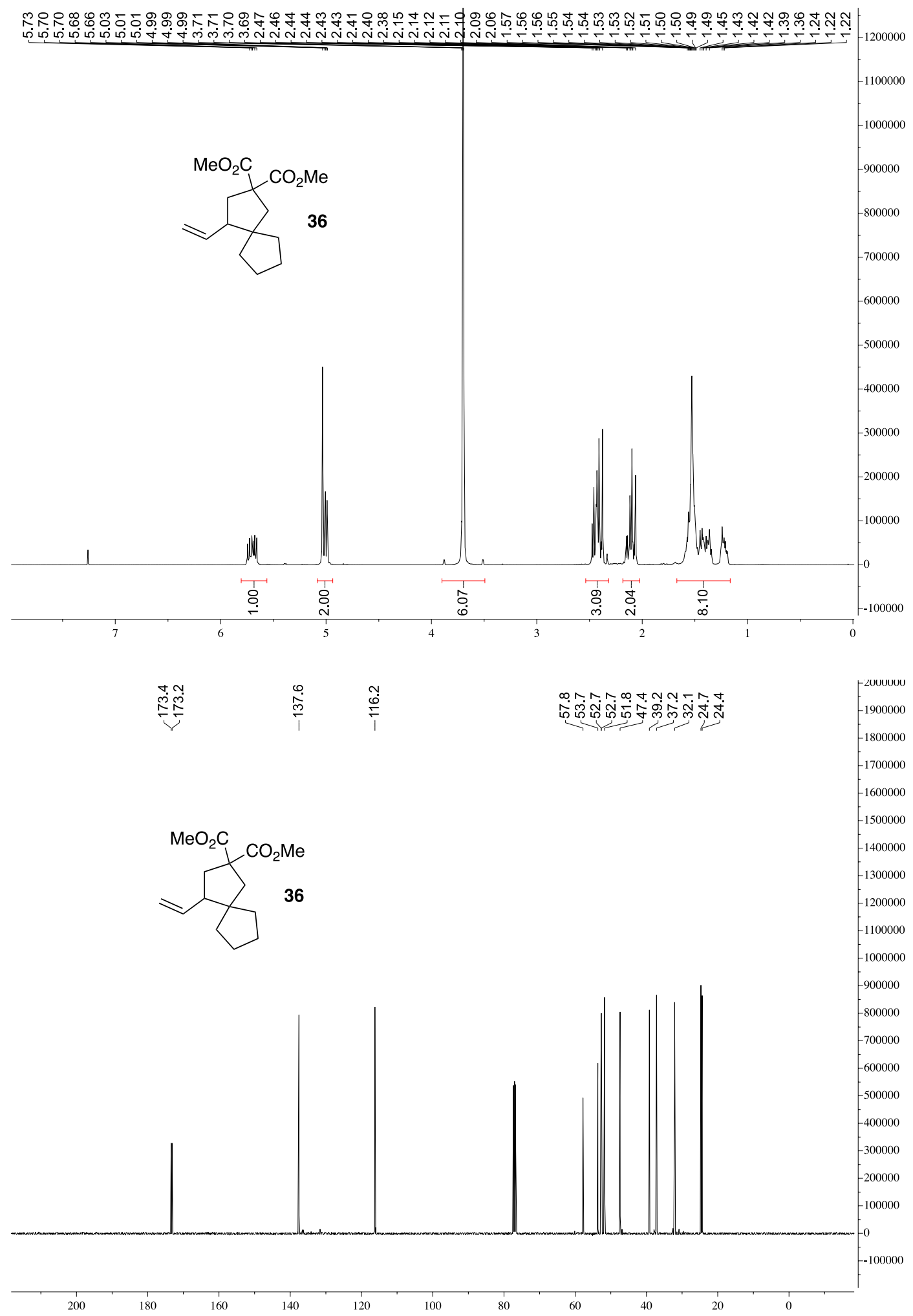




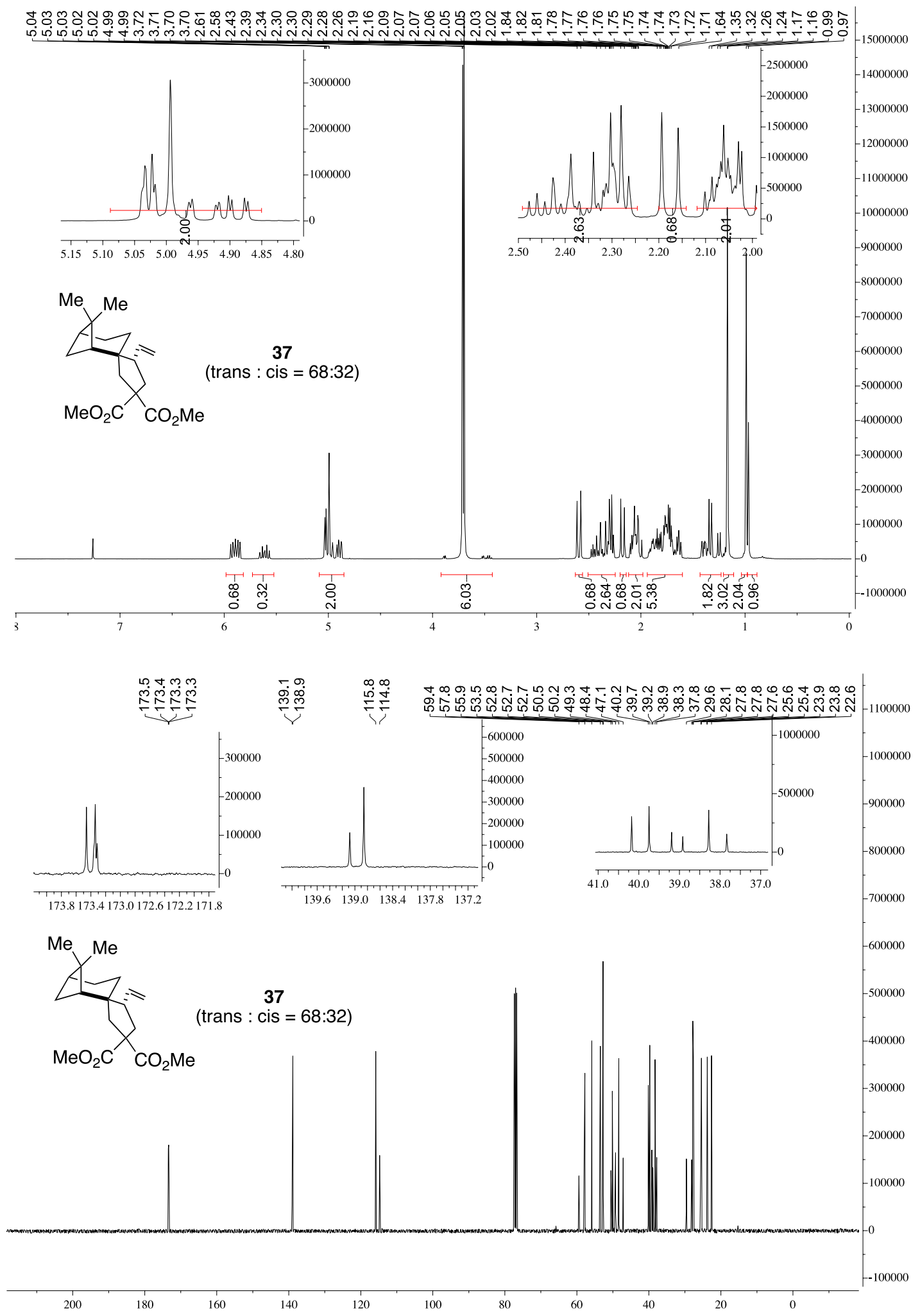




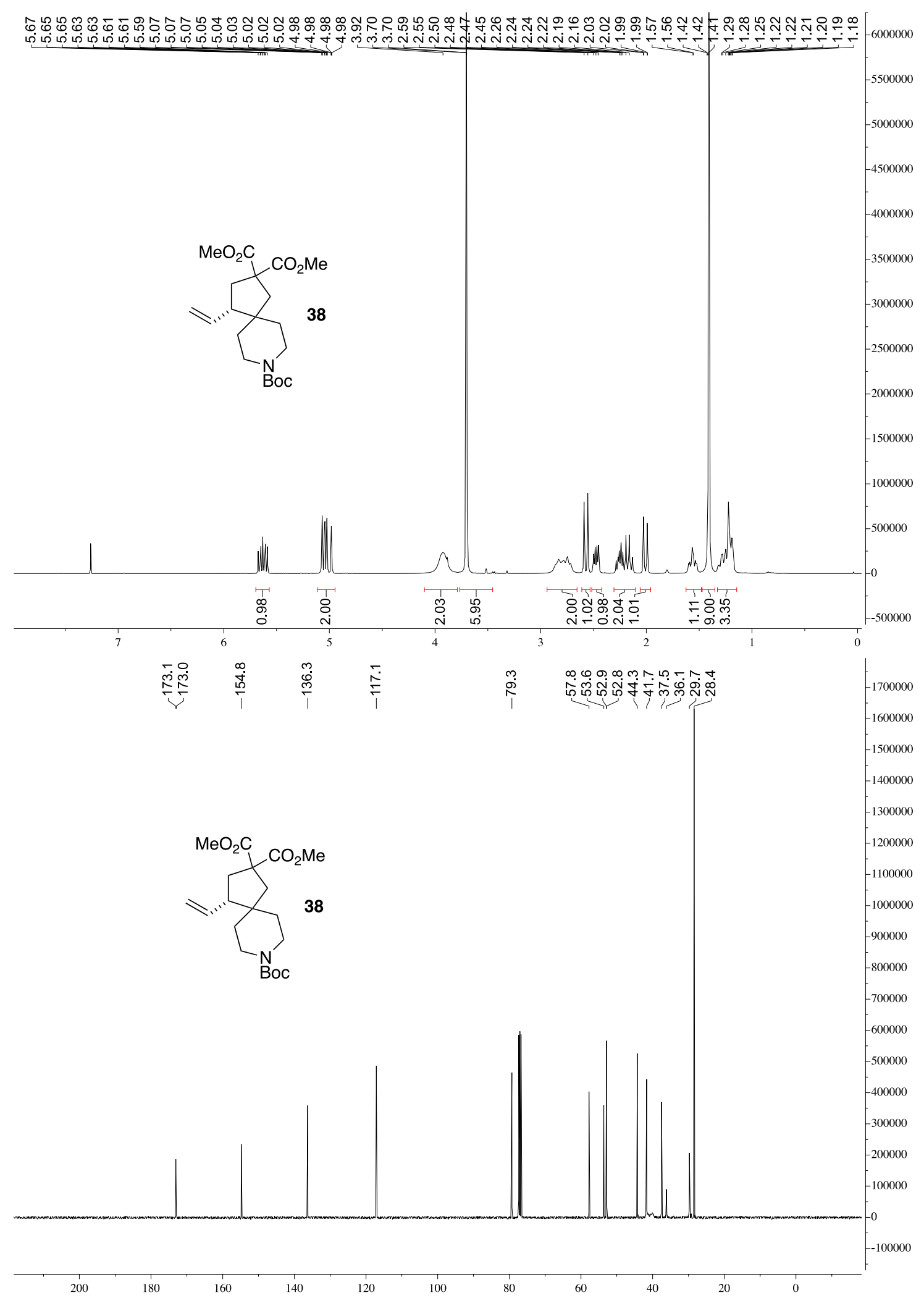




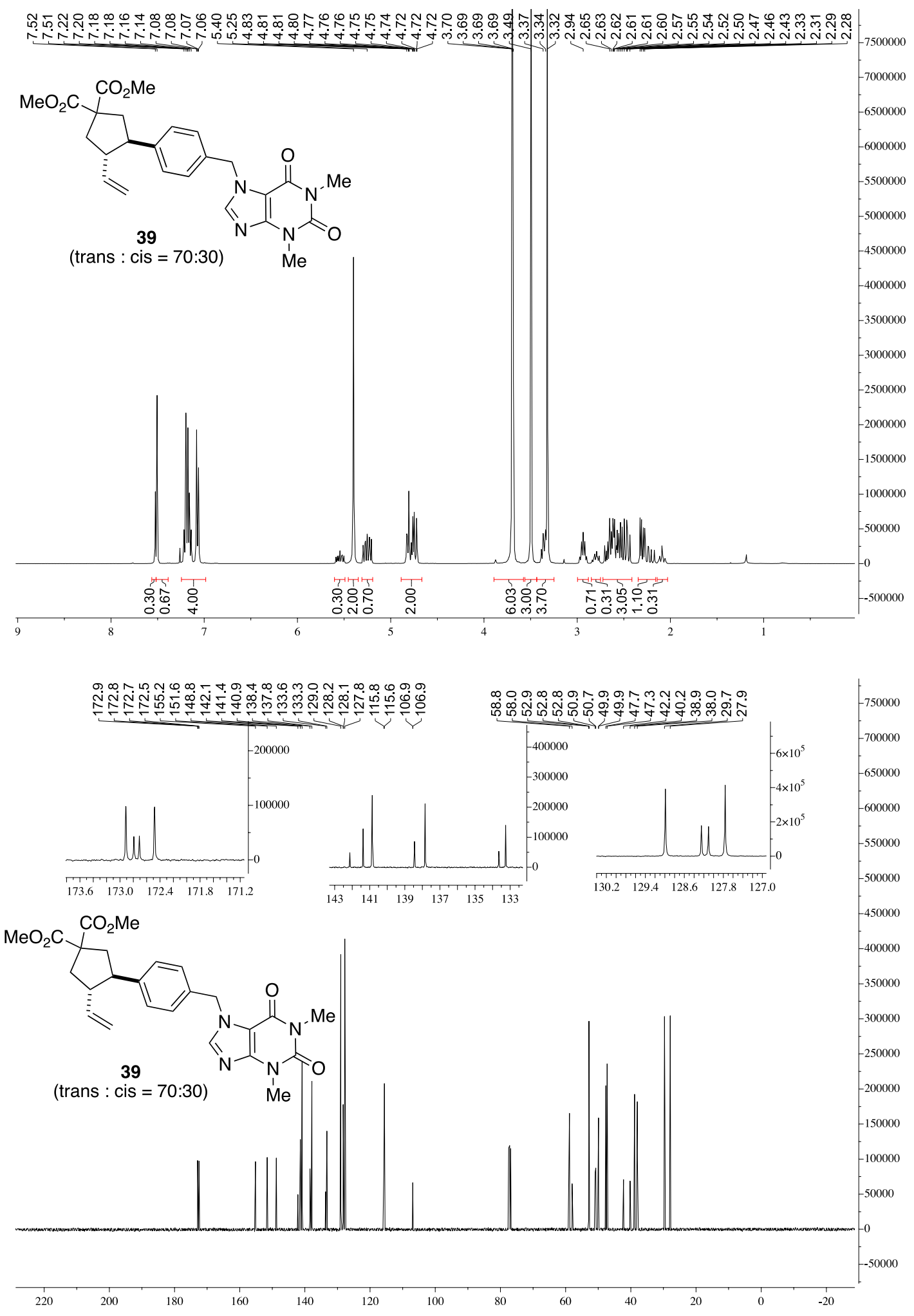




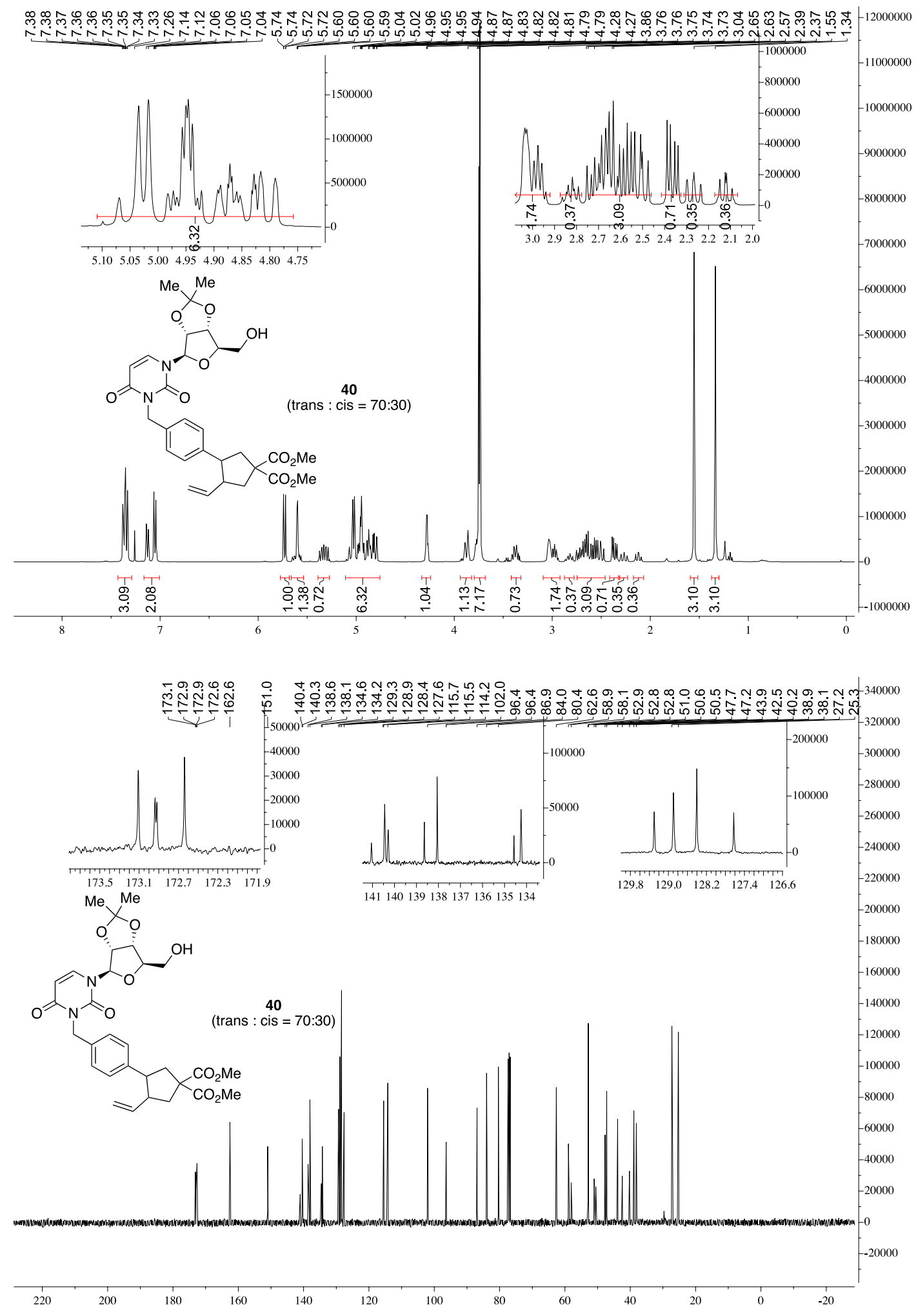



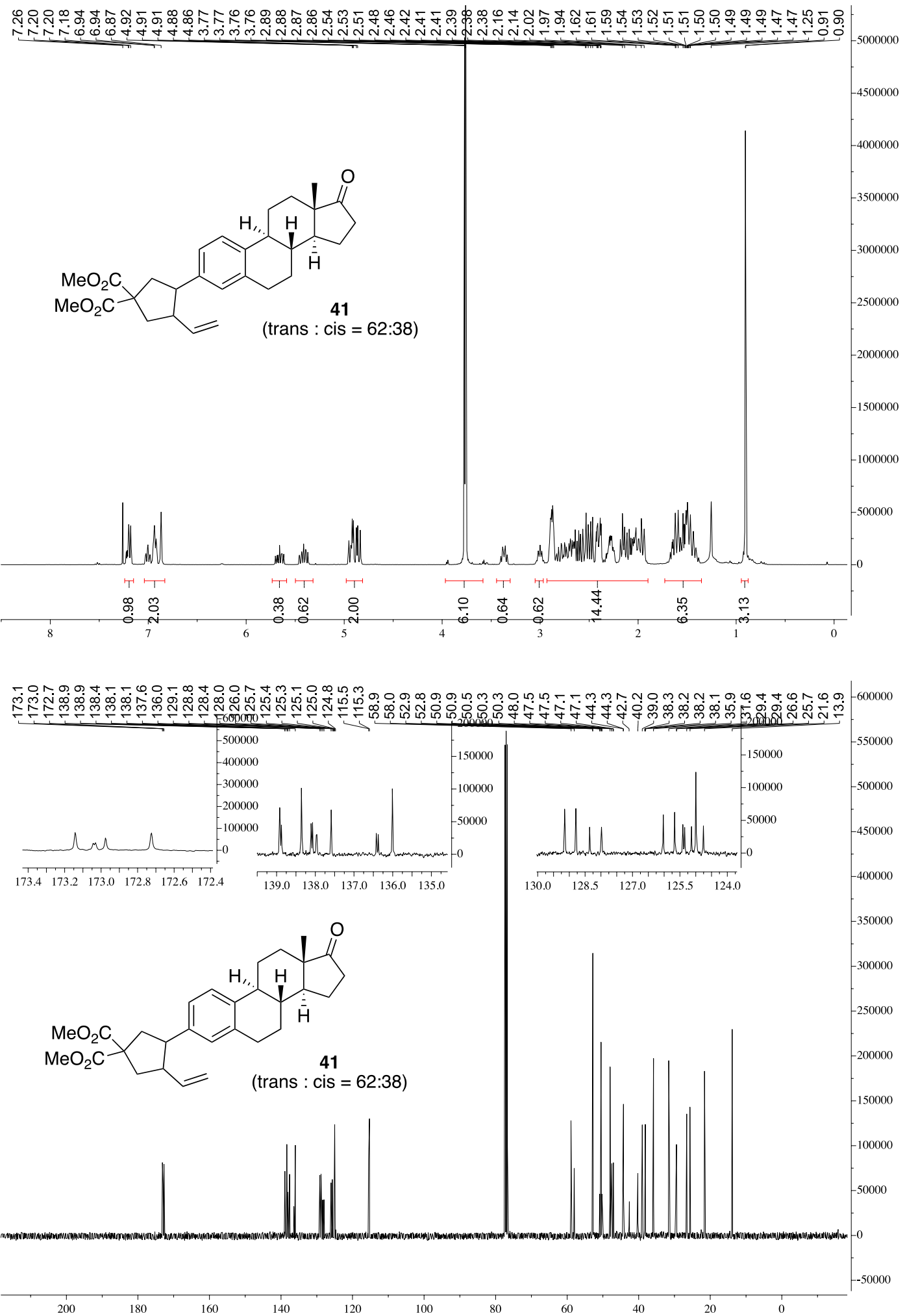

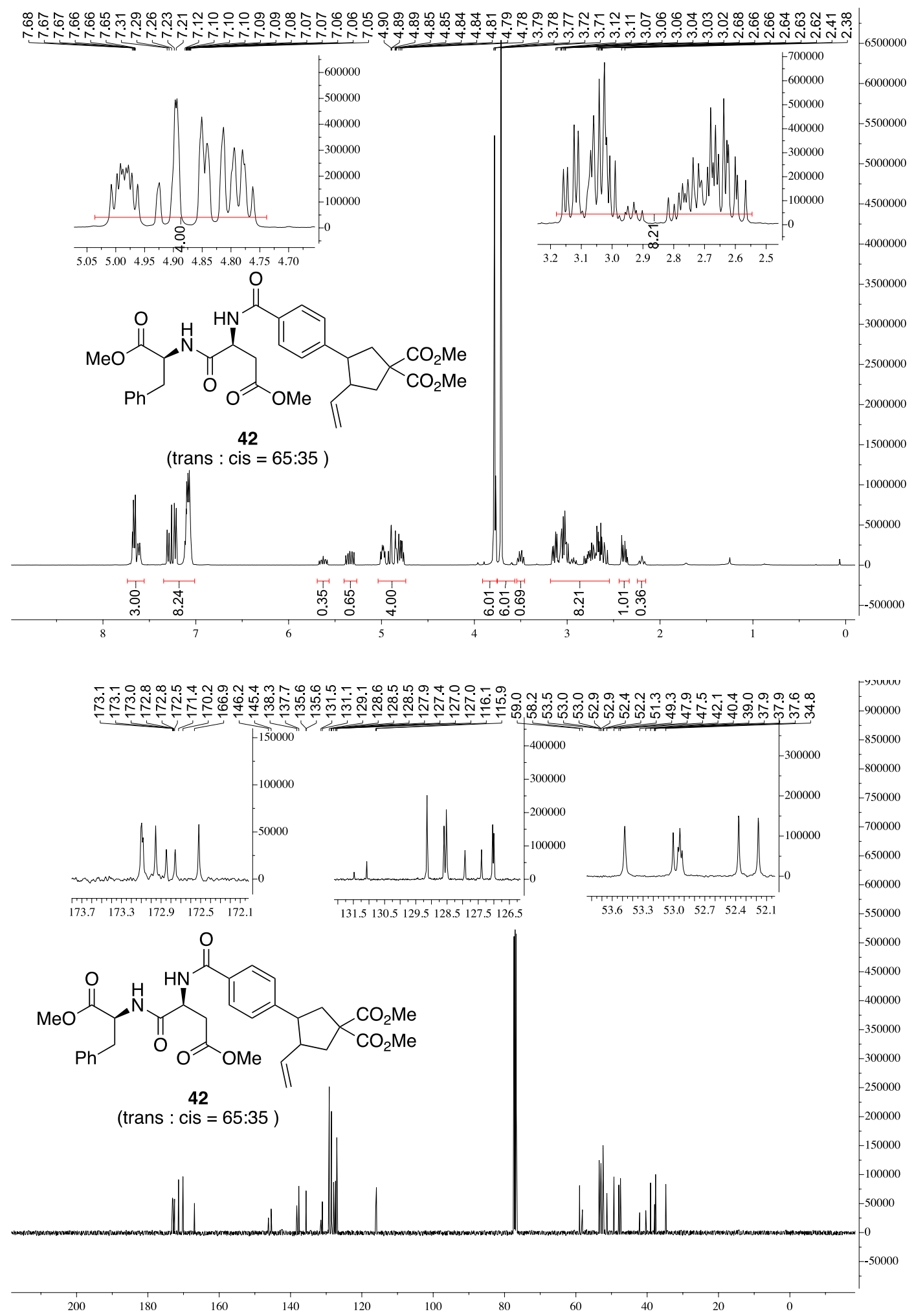

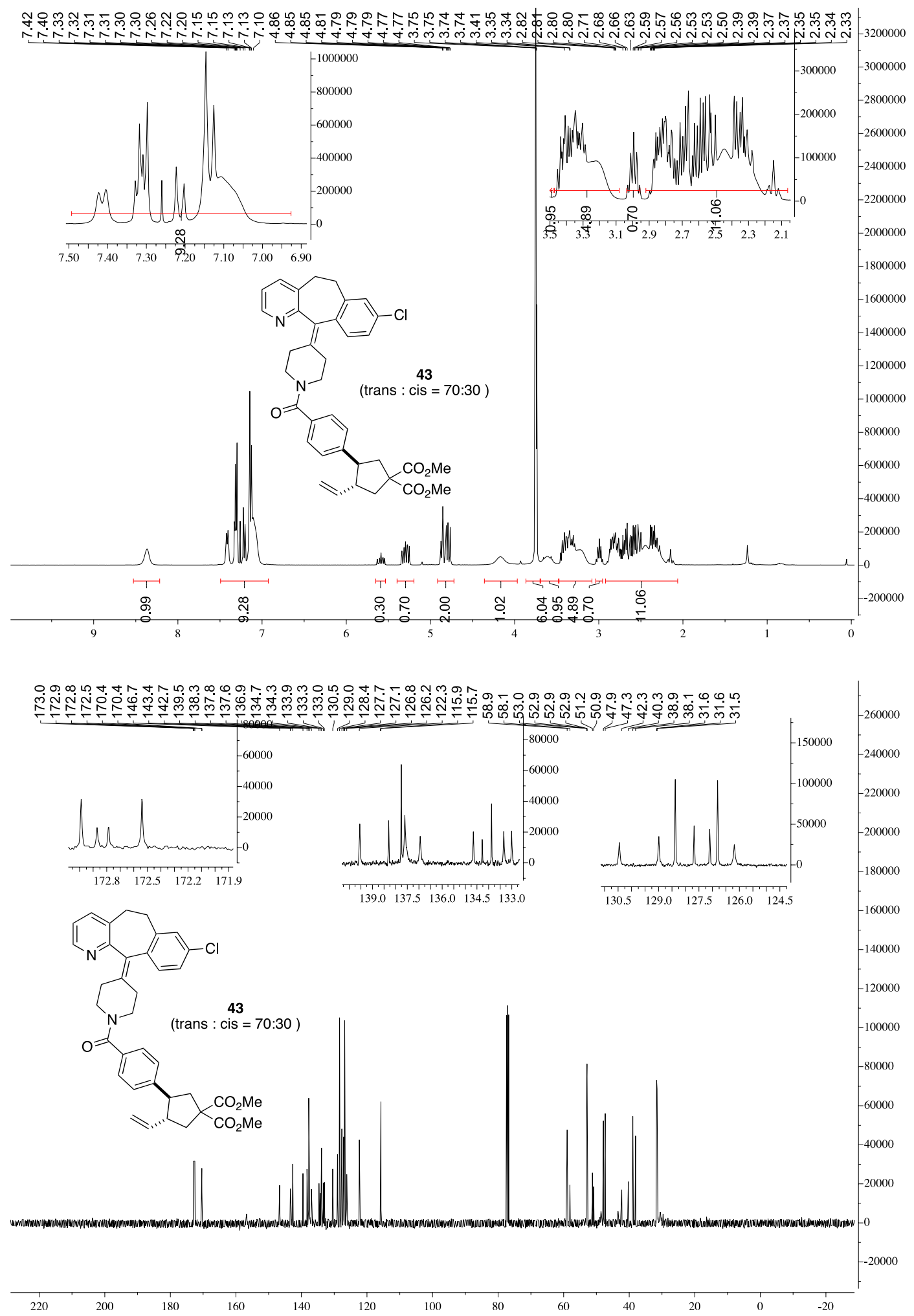


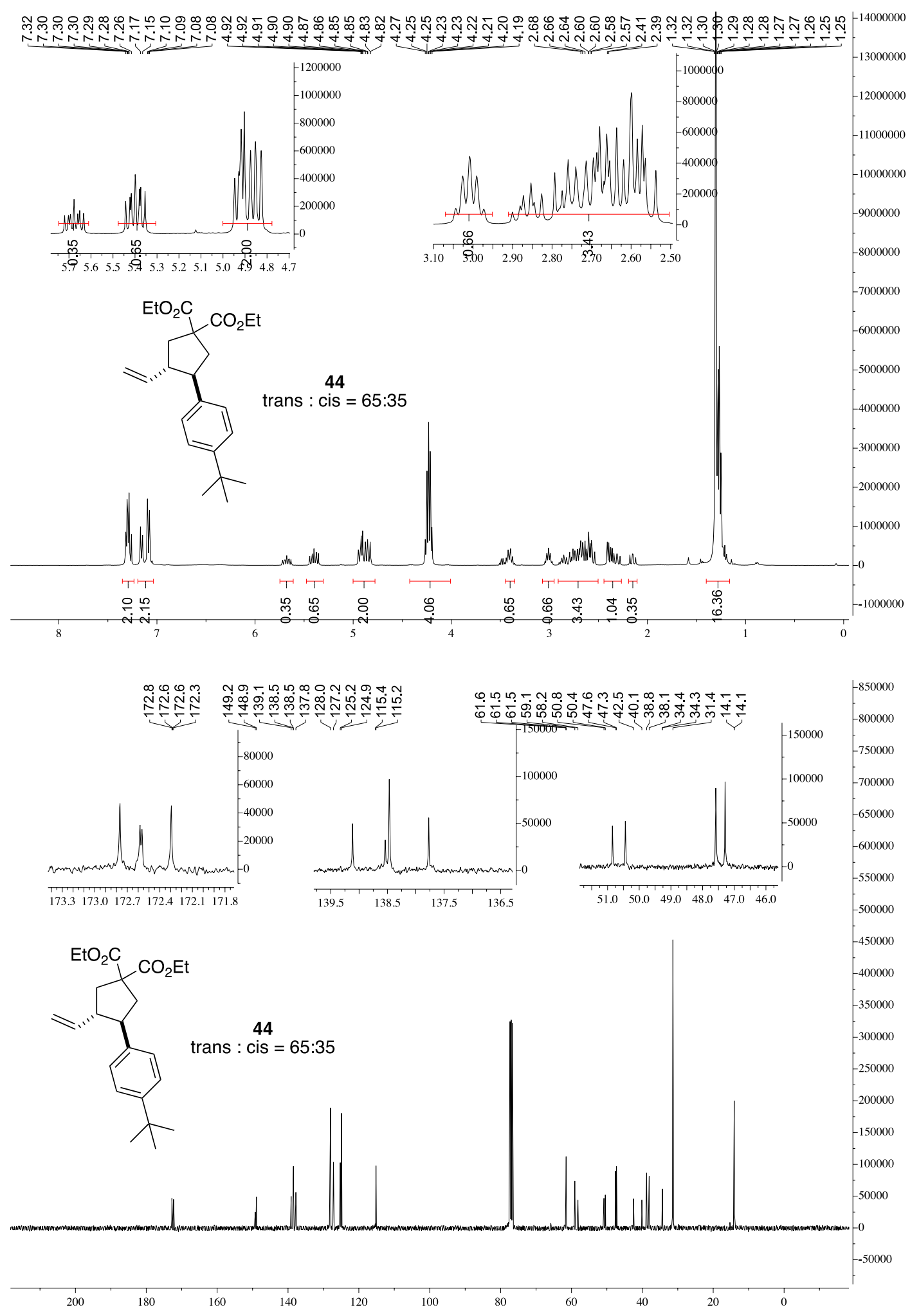




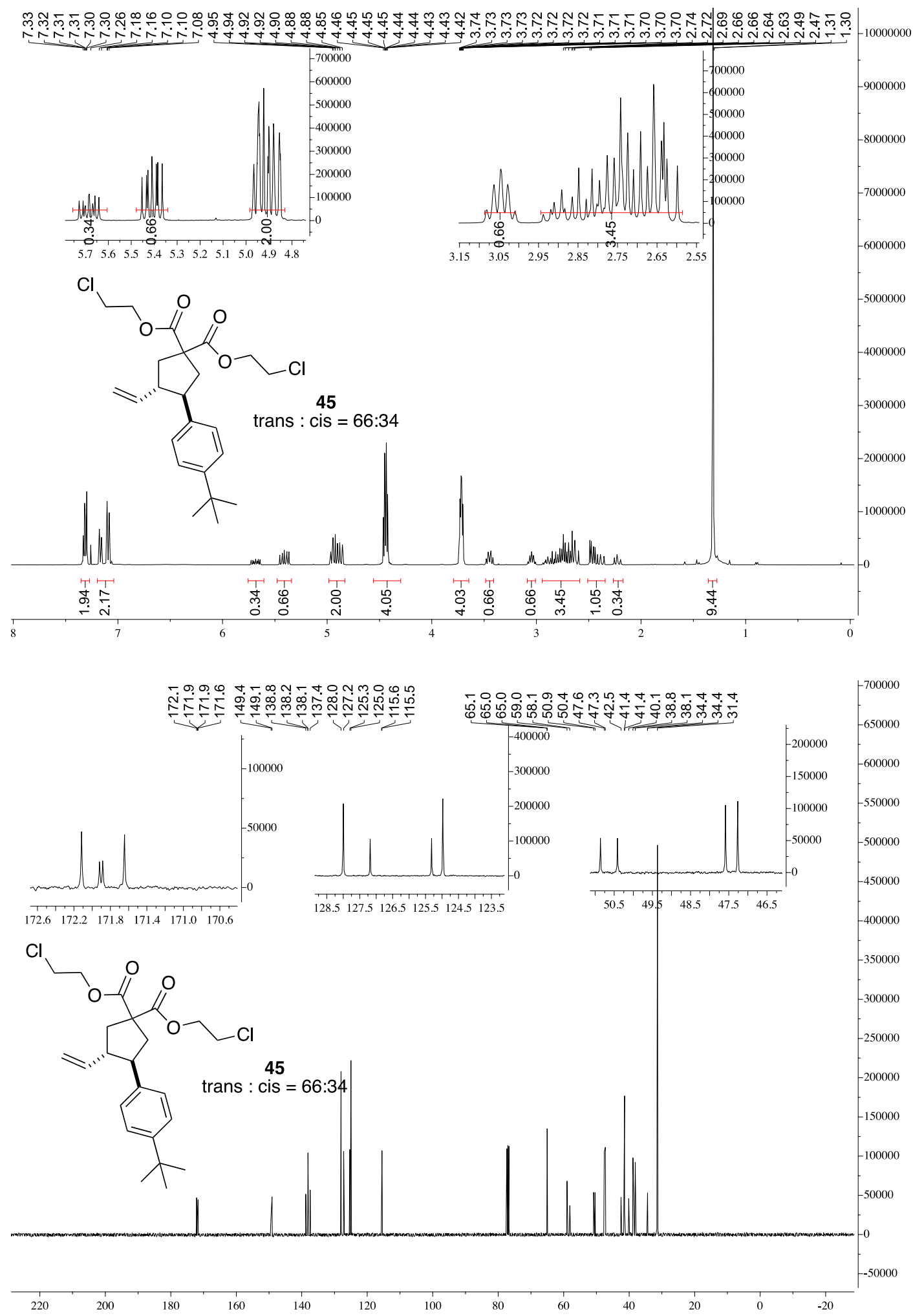




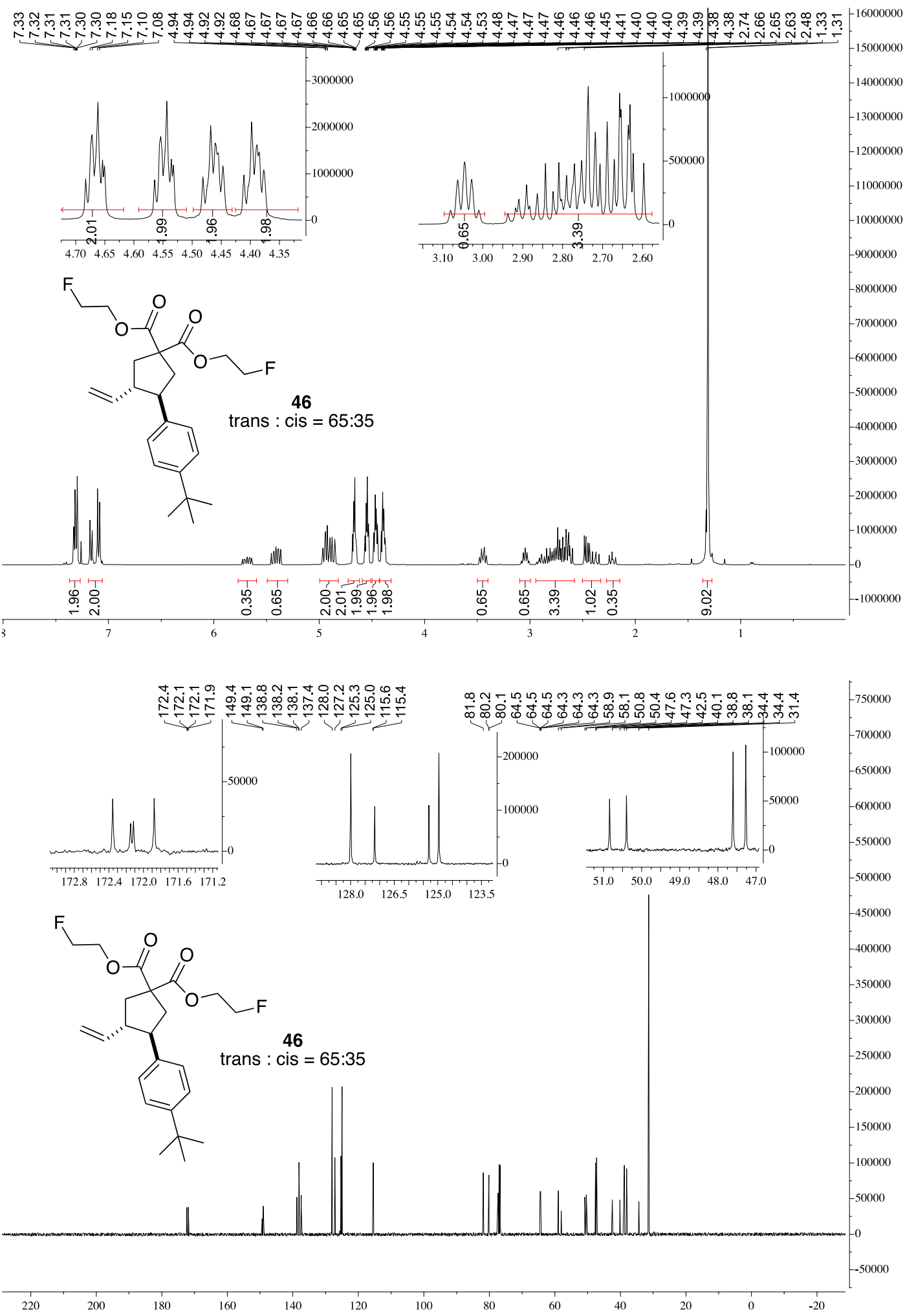




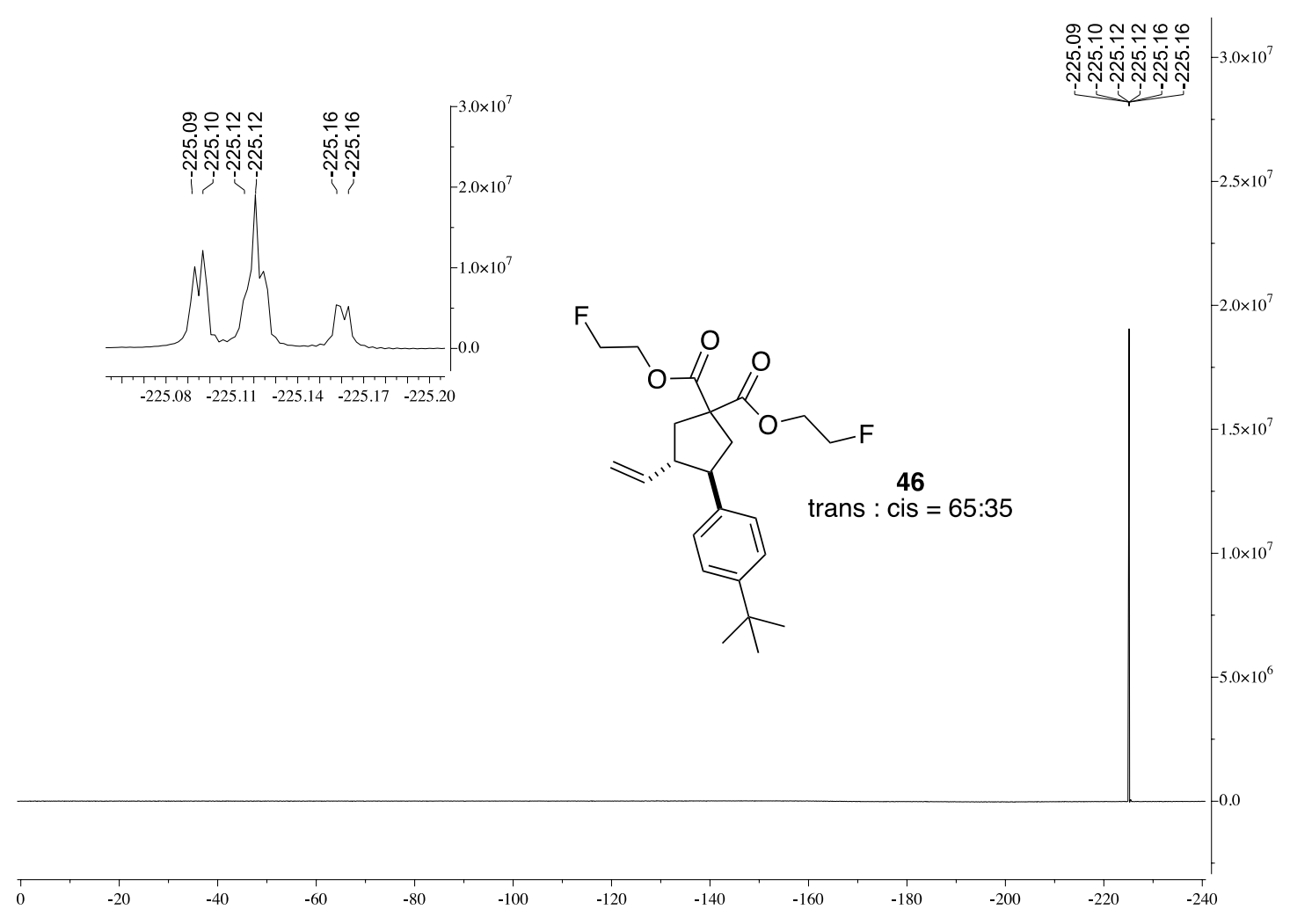




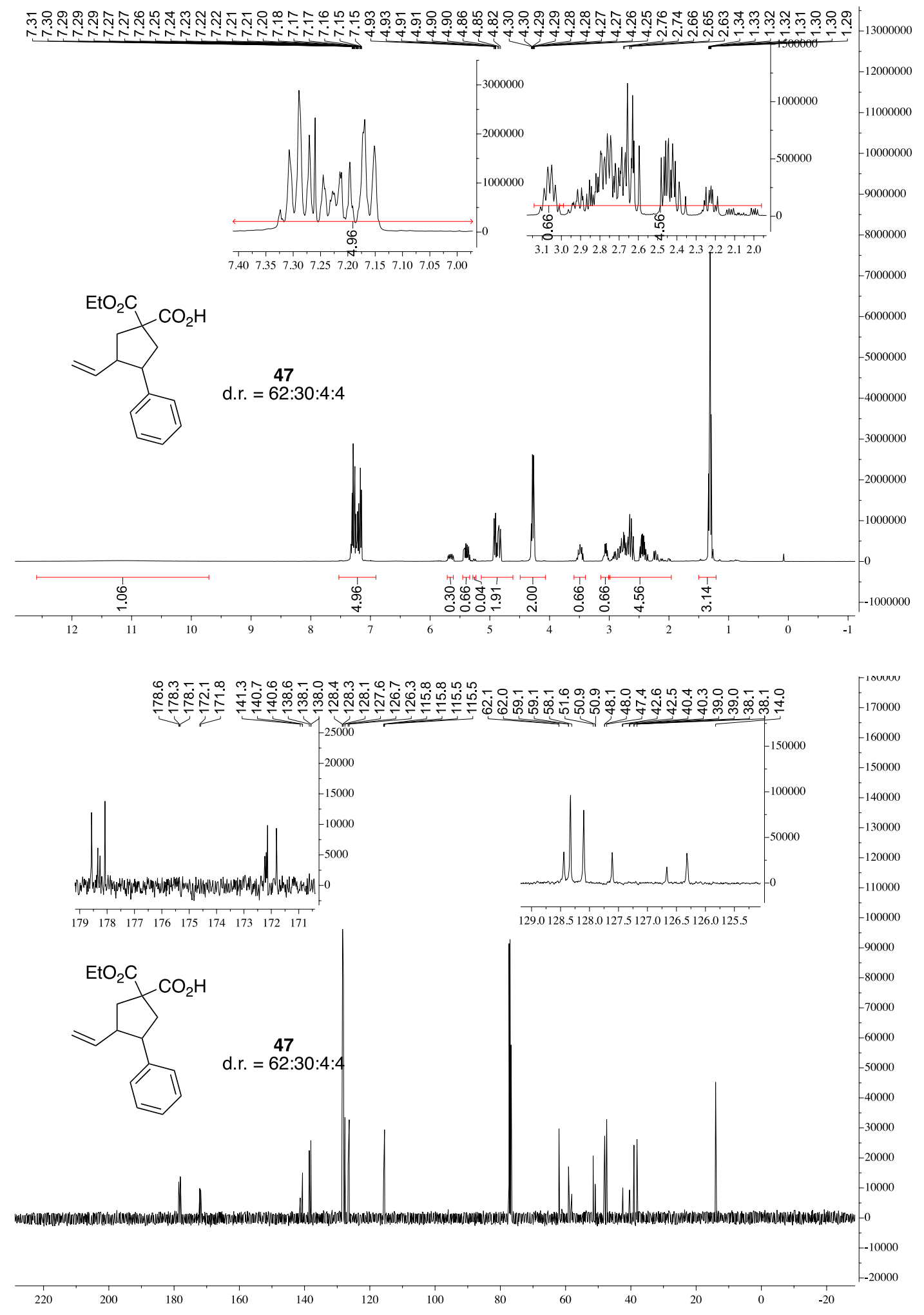



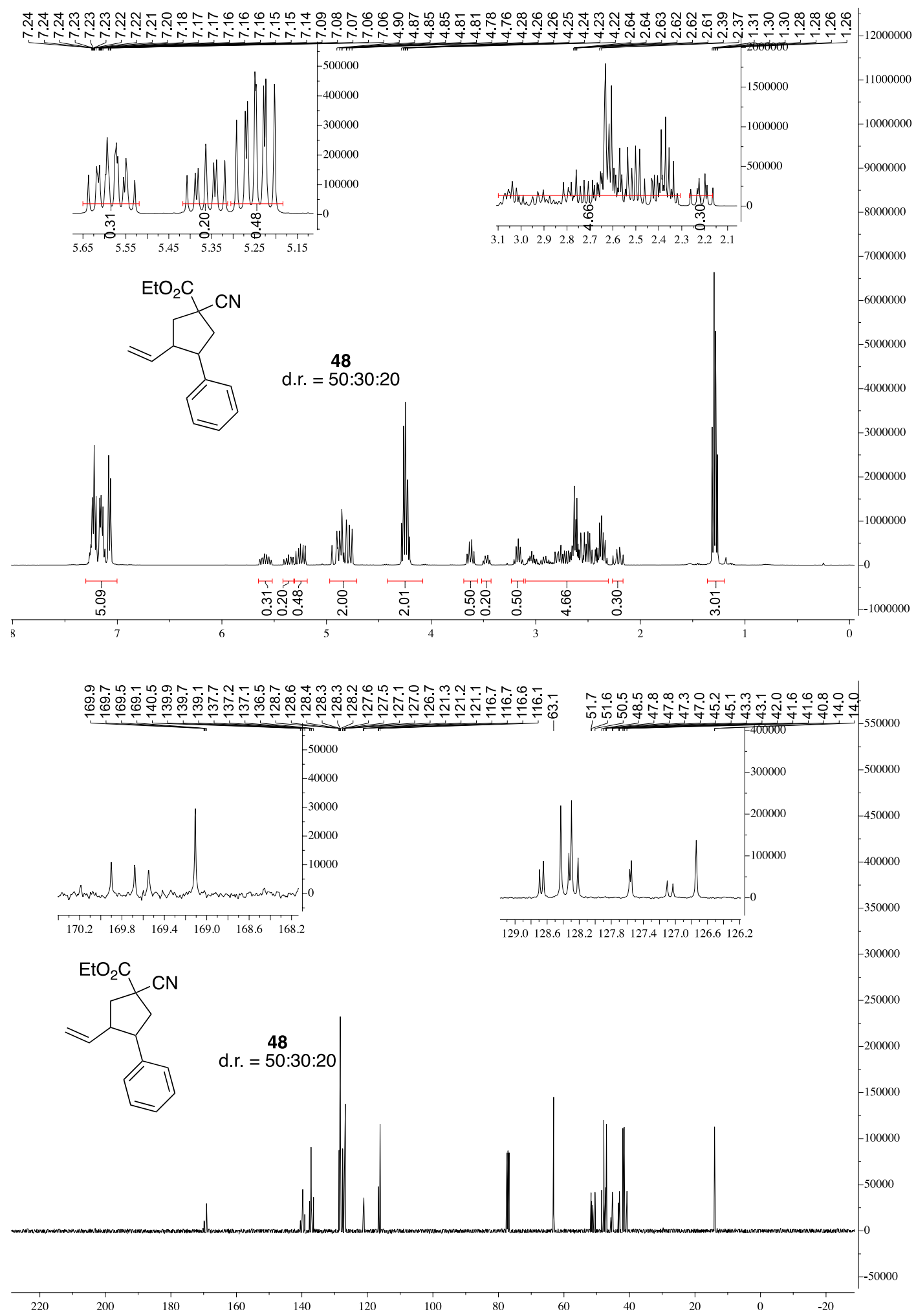

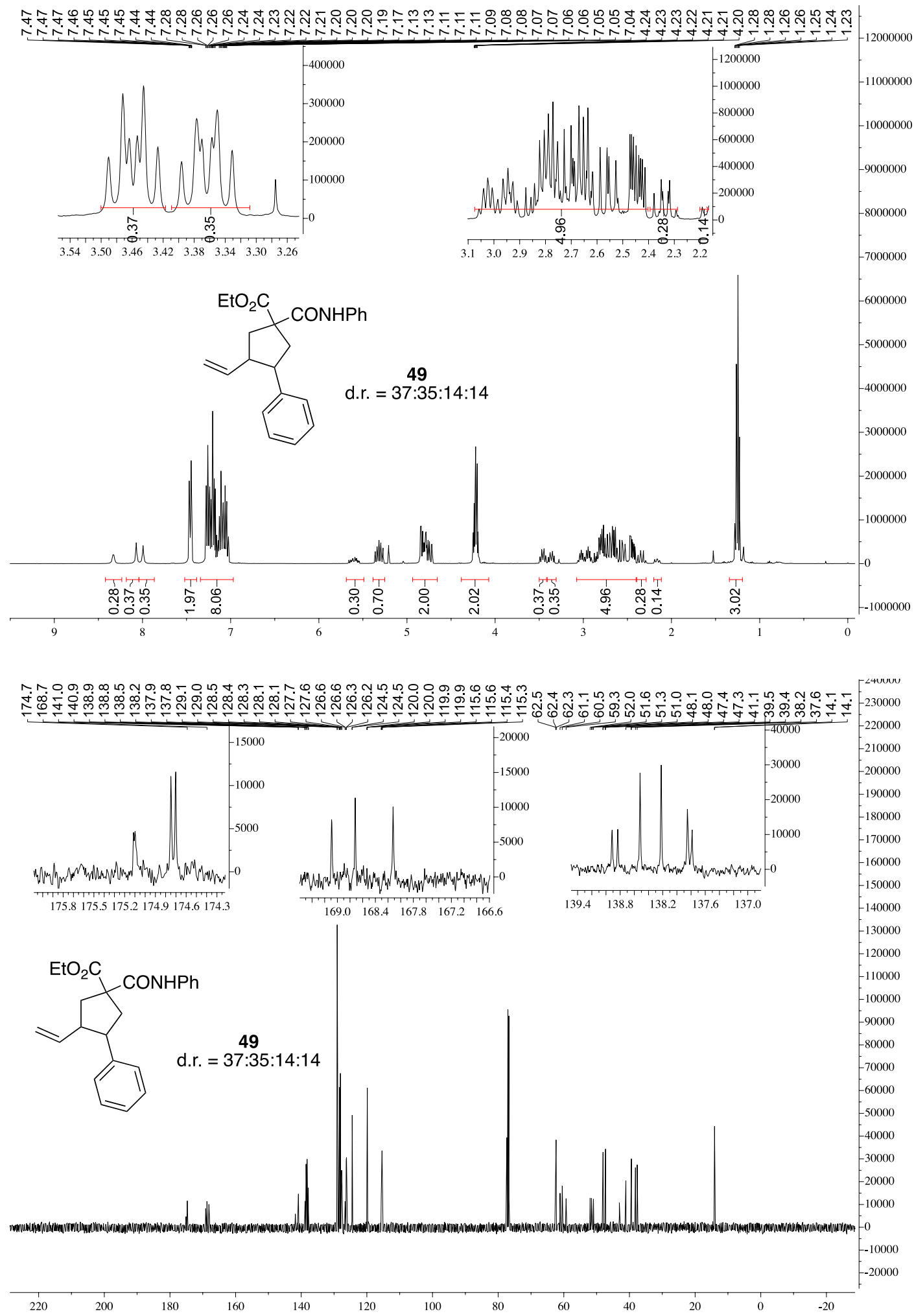

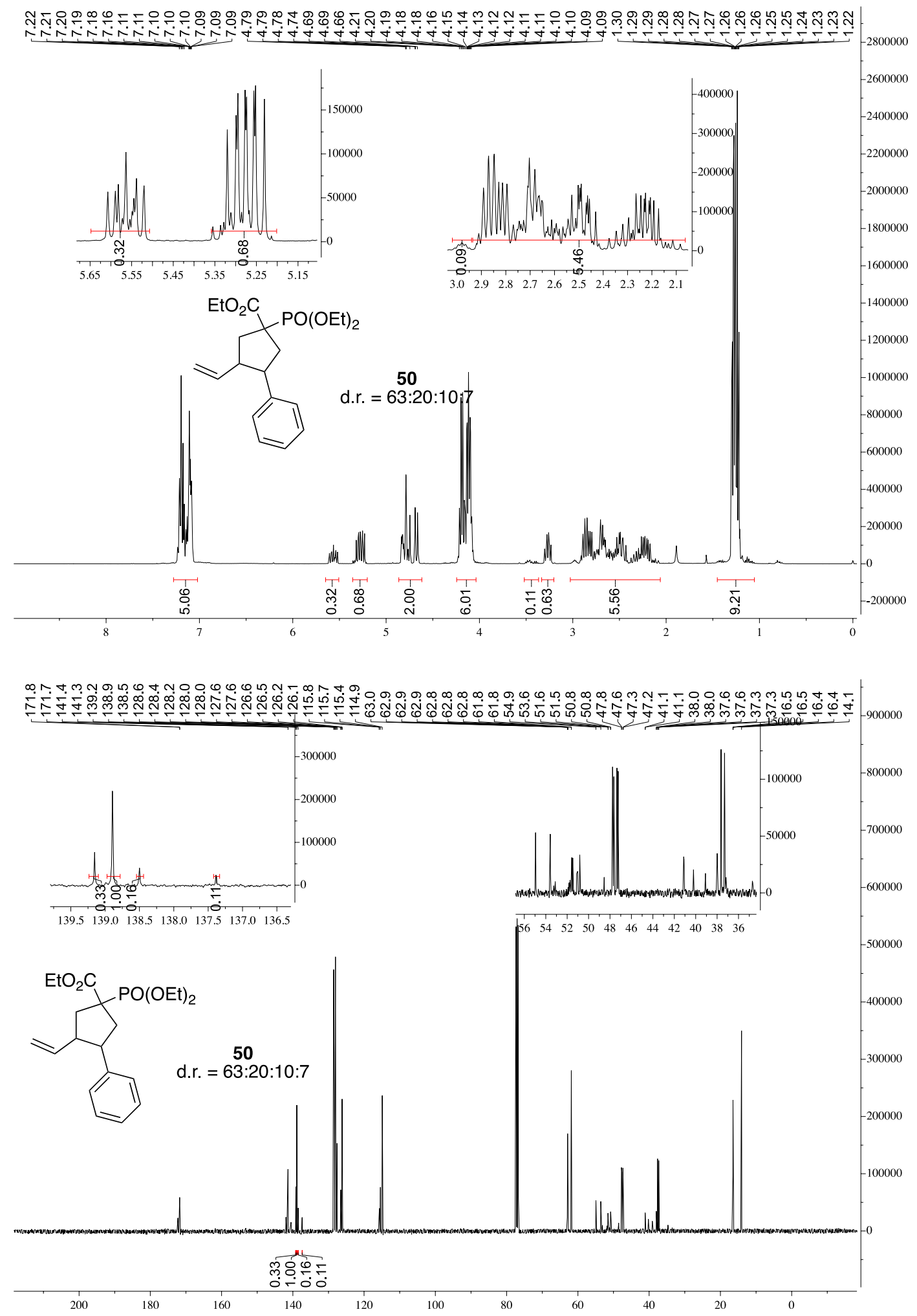


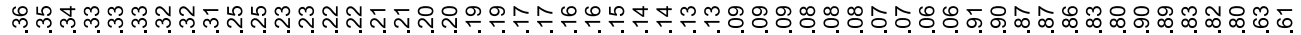

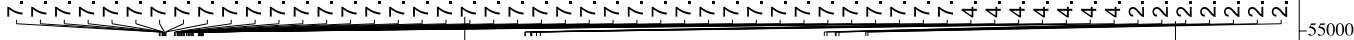
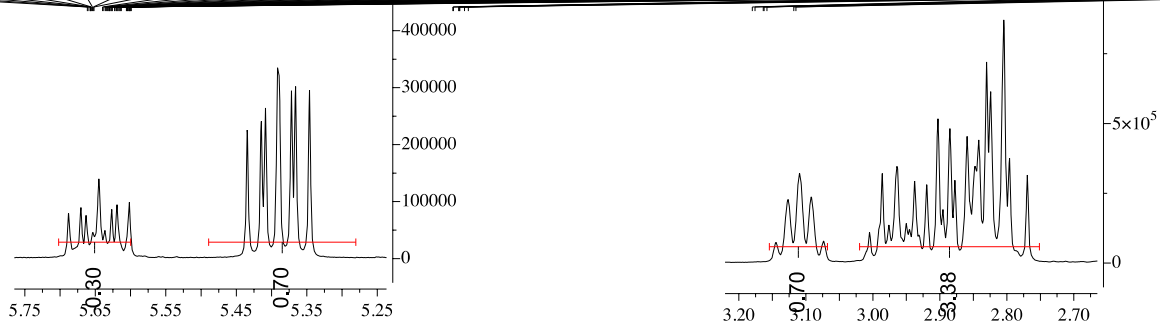

500000

$\mathrm{PhO}_{2} \mathrm{C}$
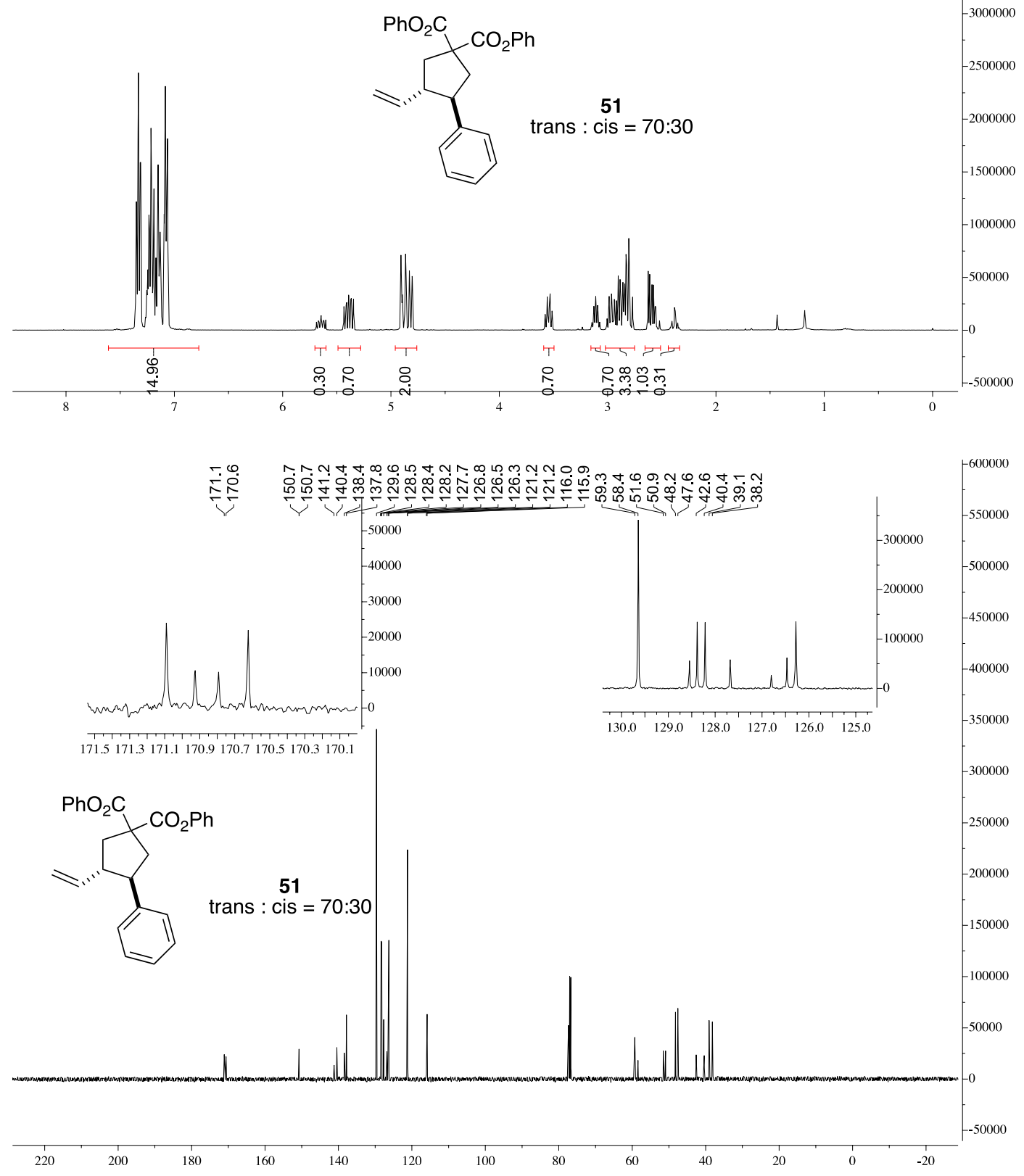


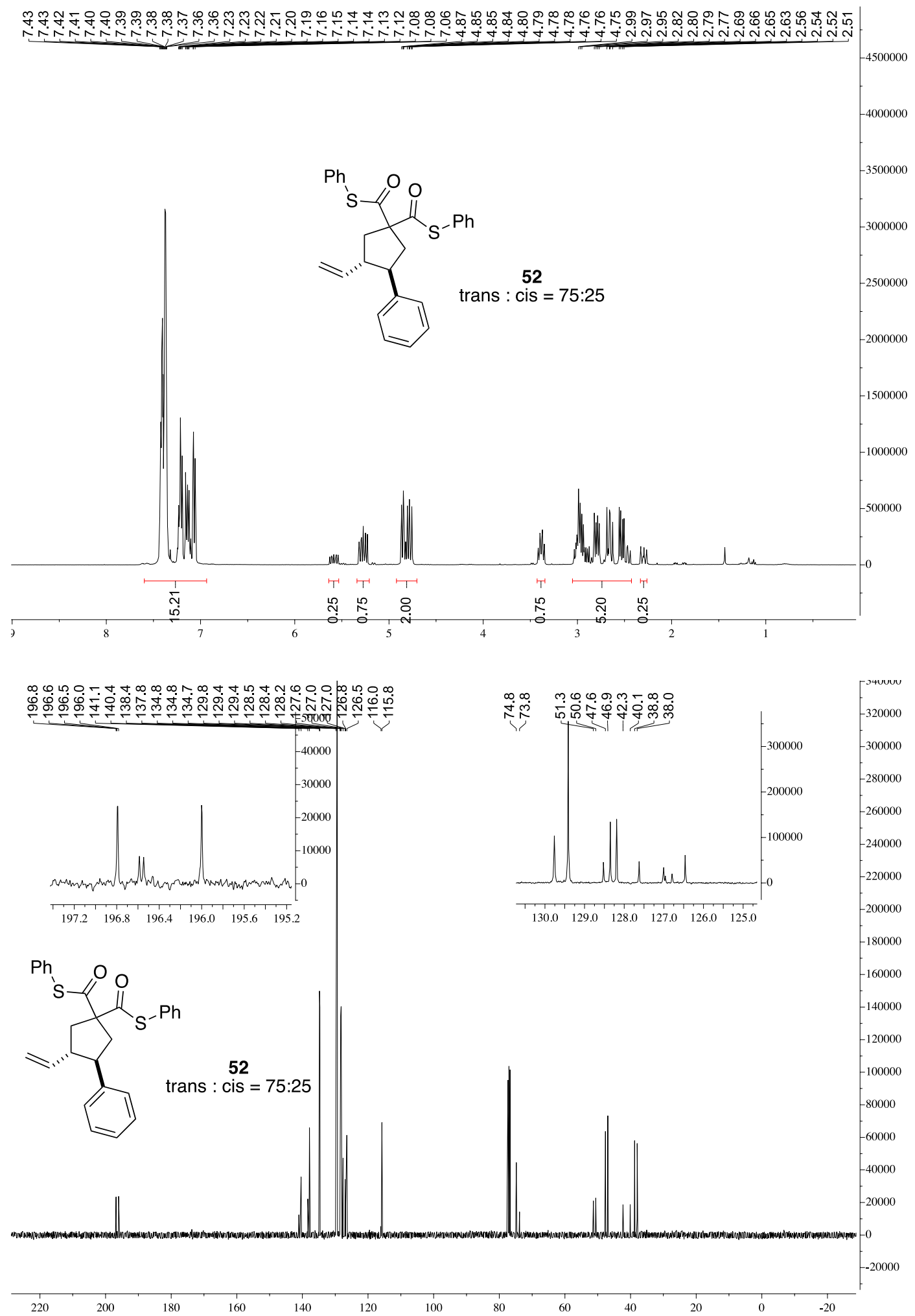




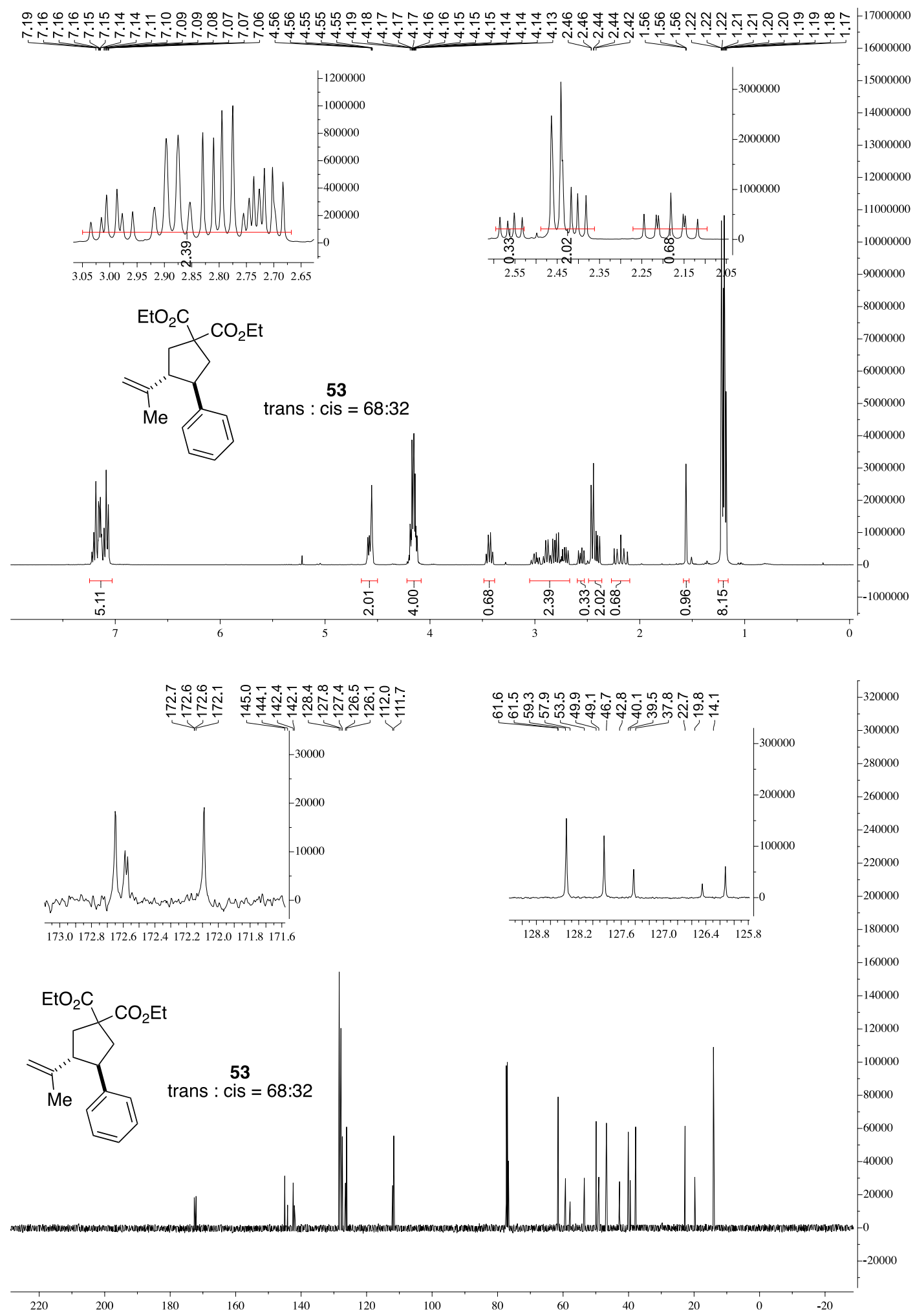




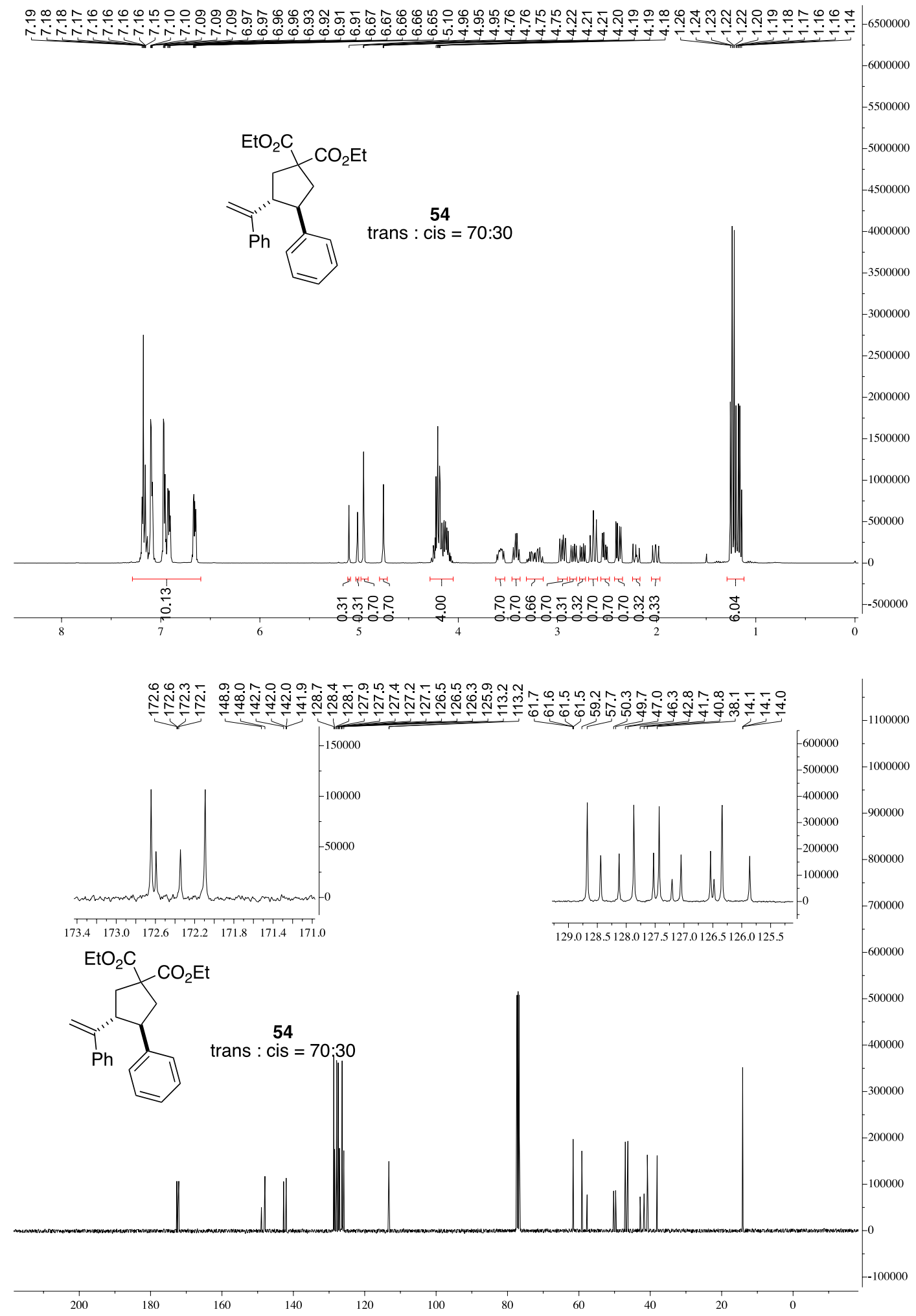




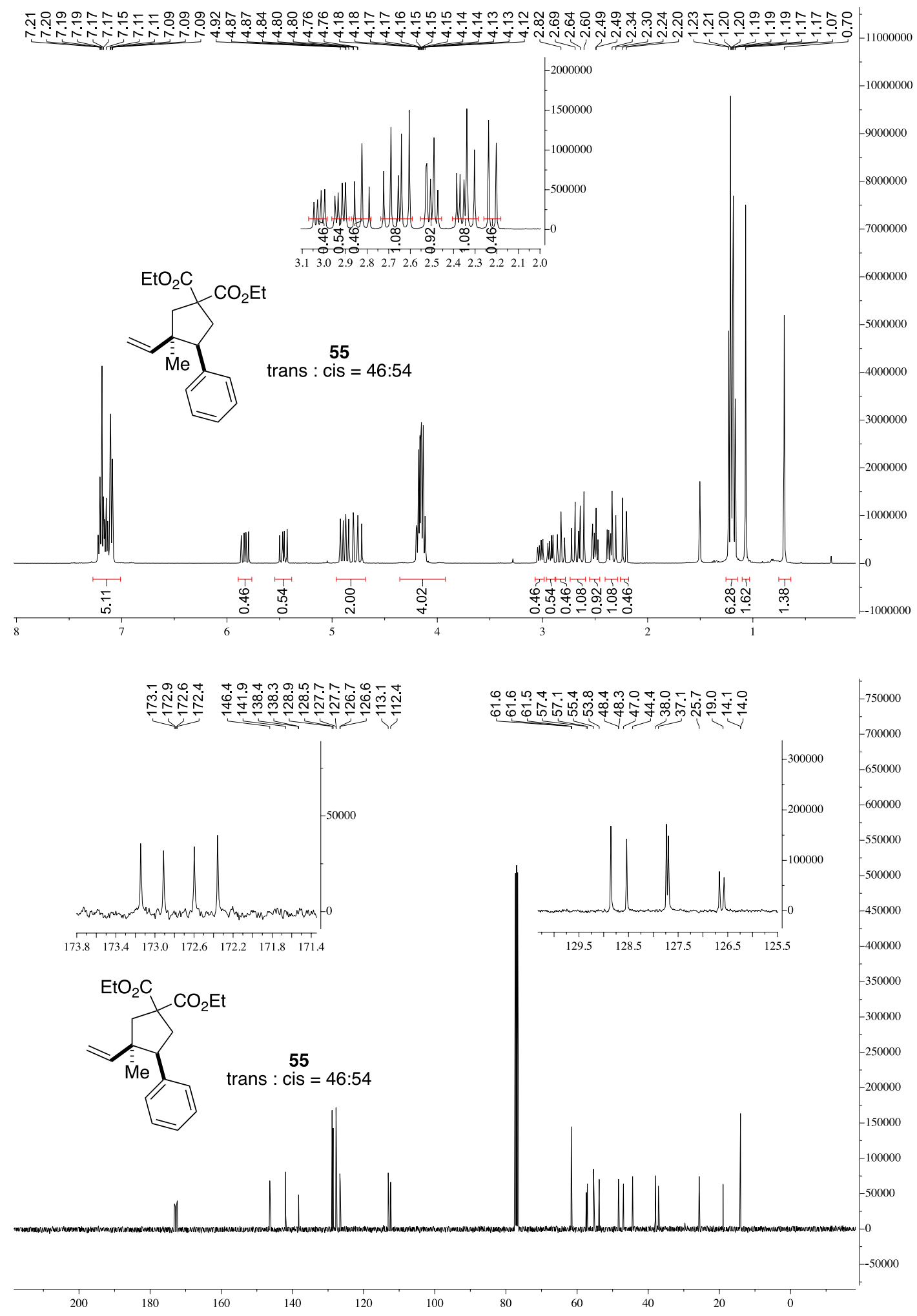




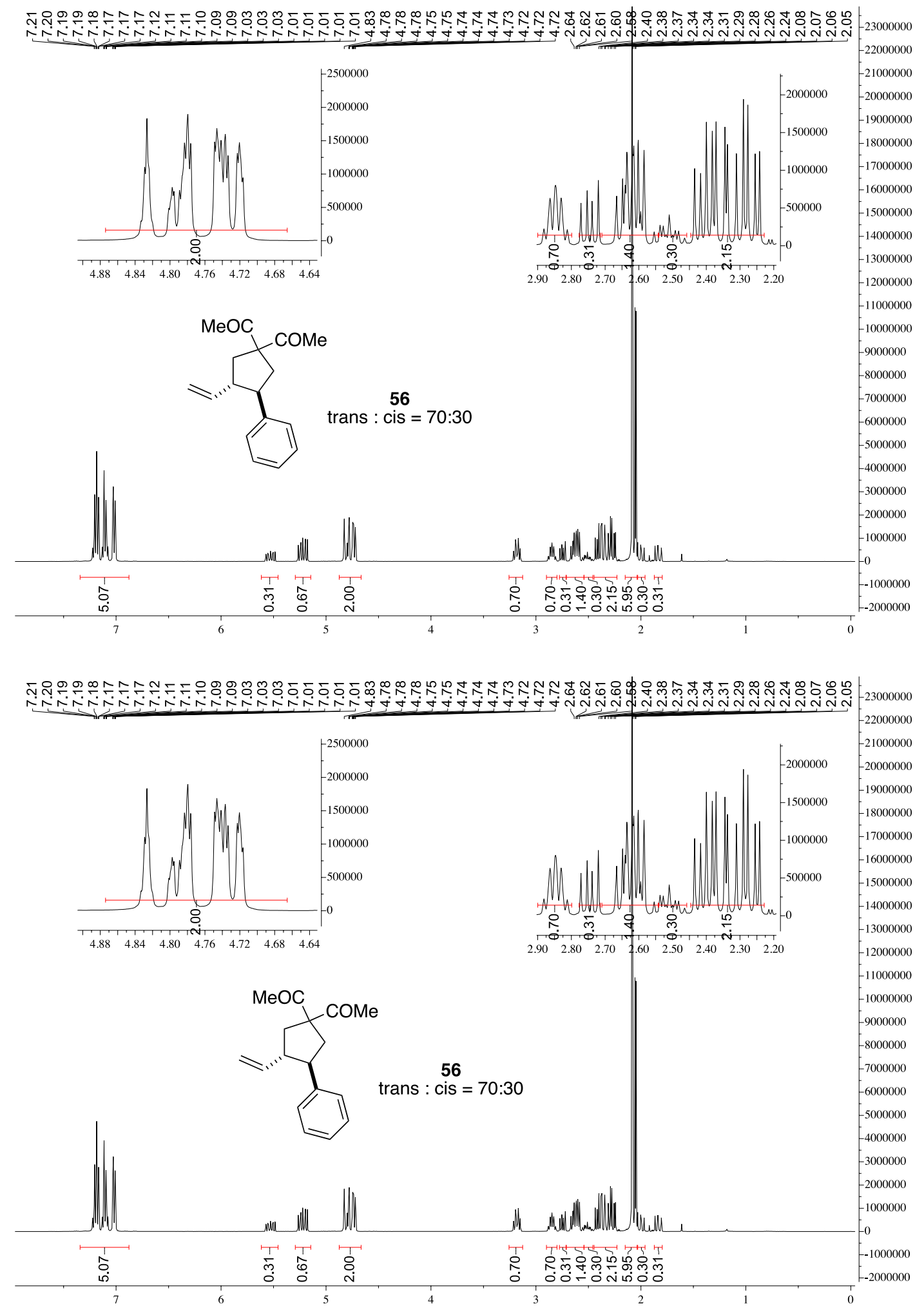



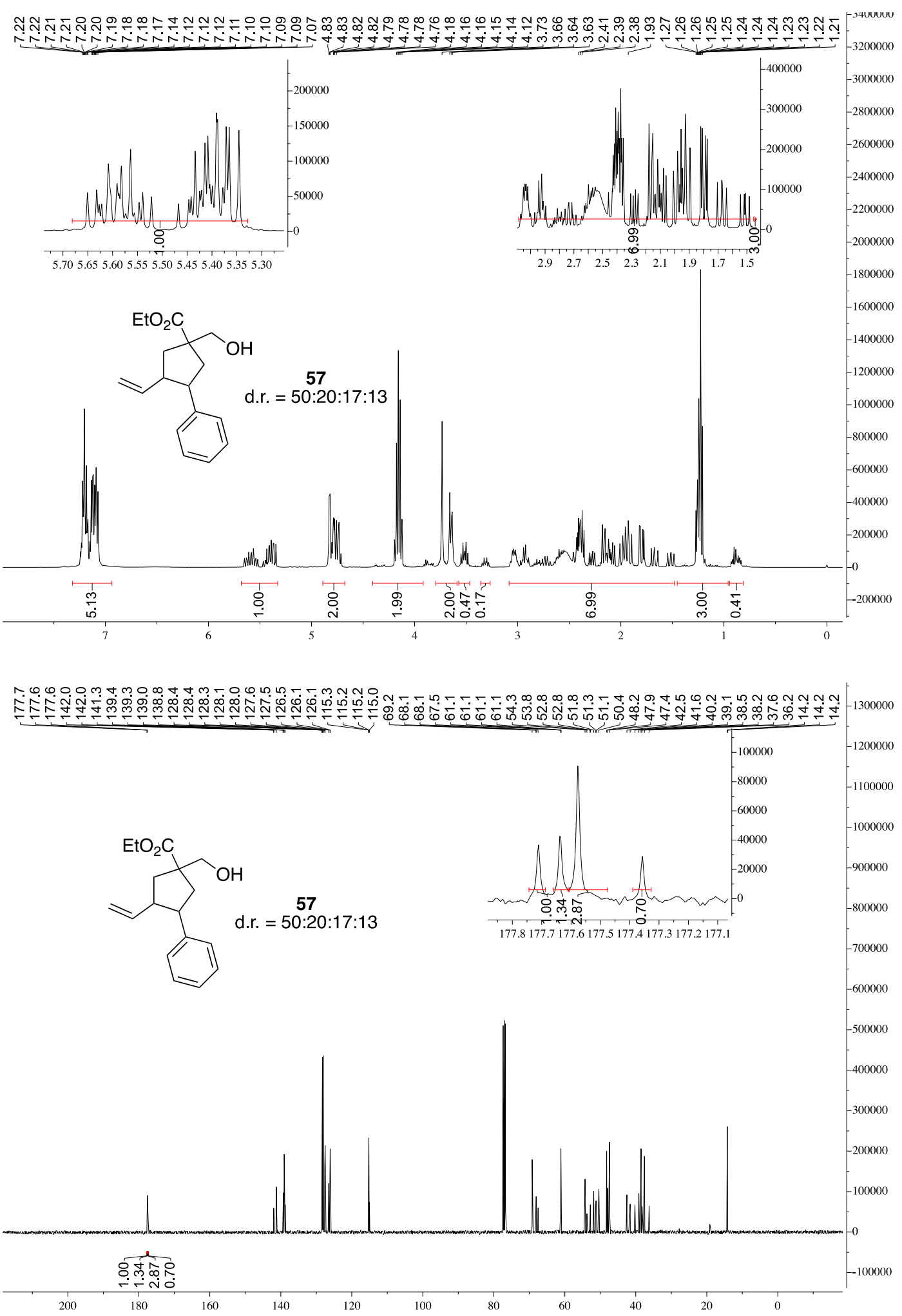

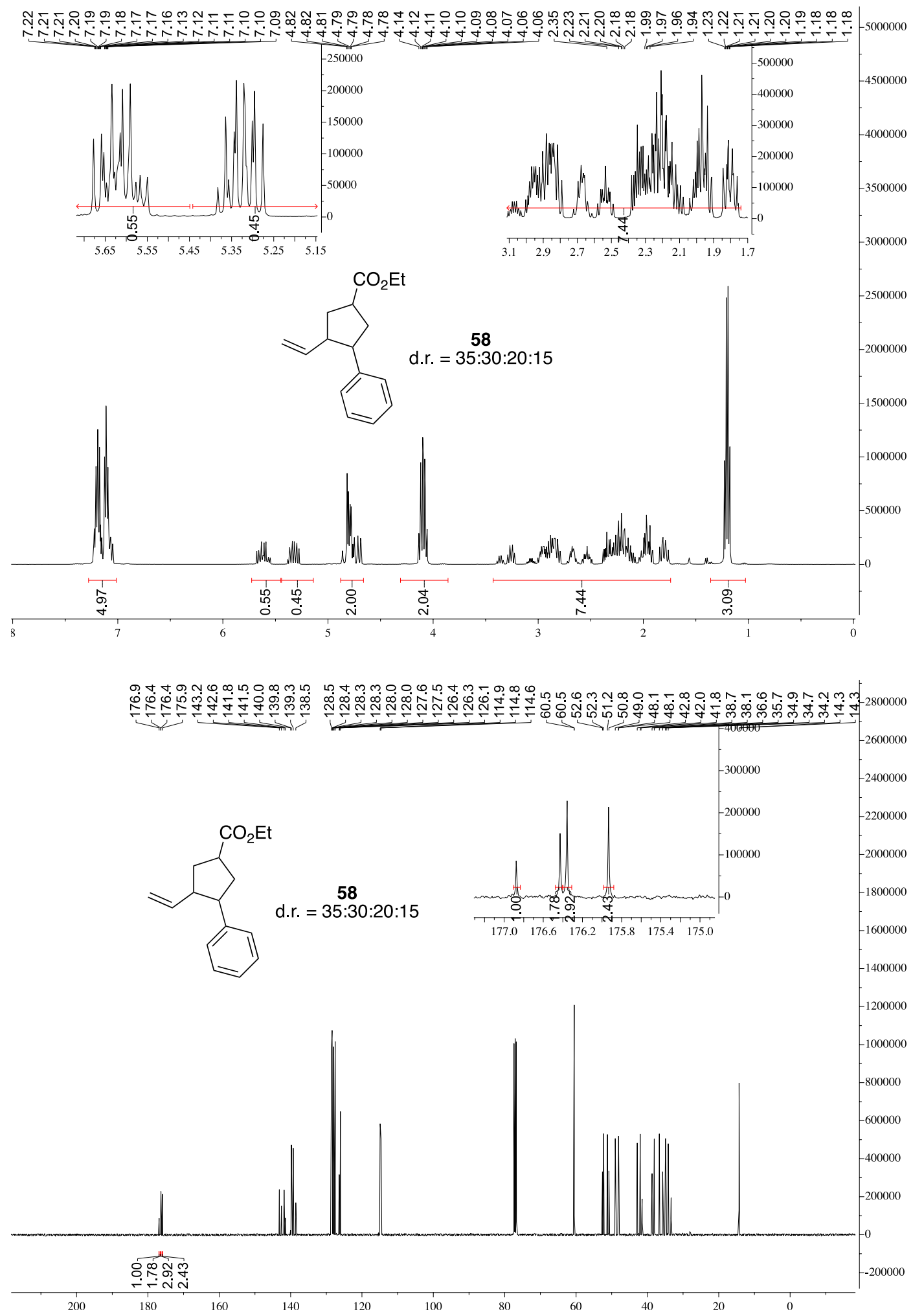


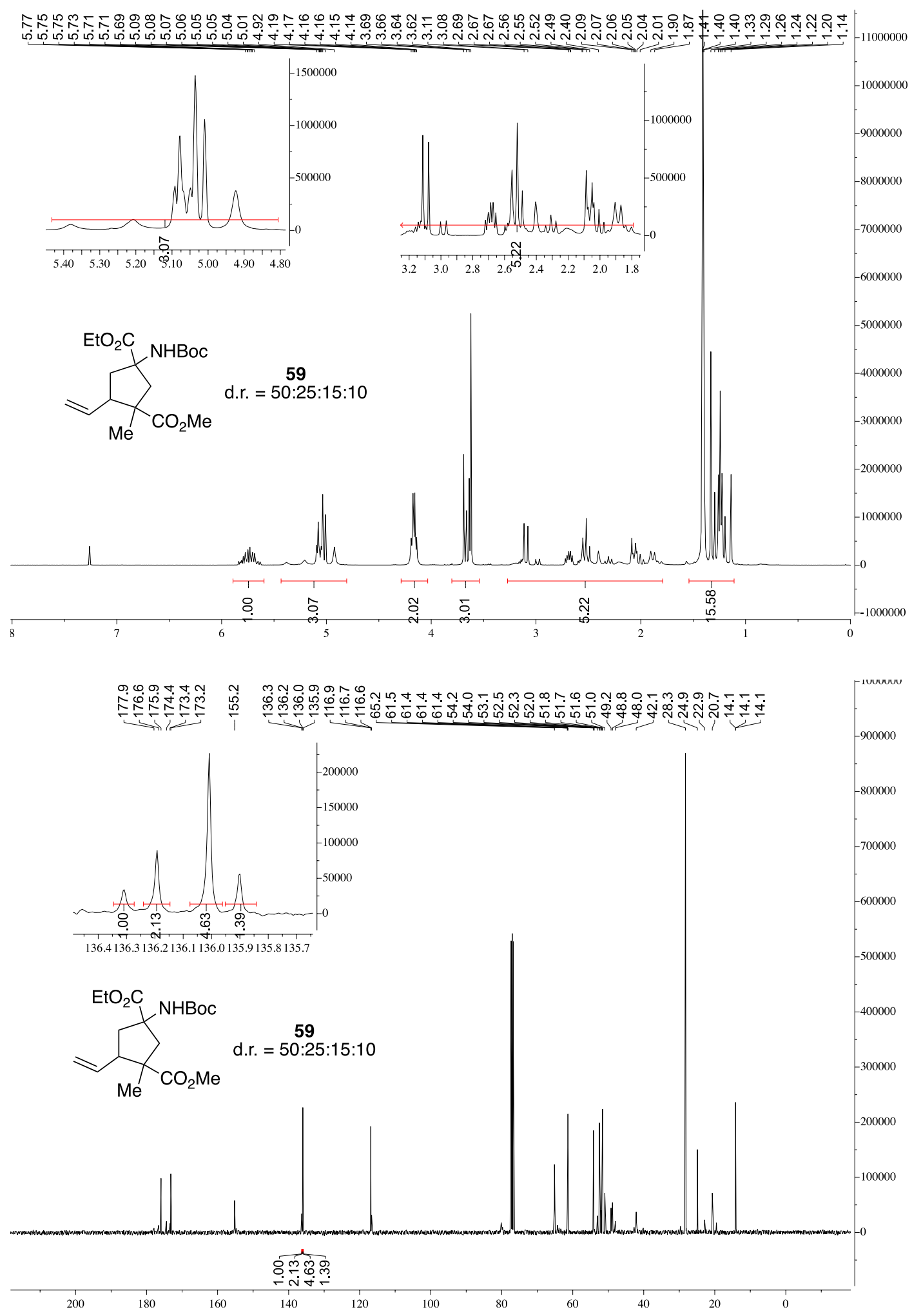




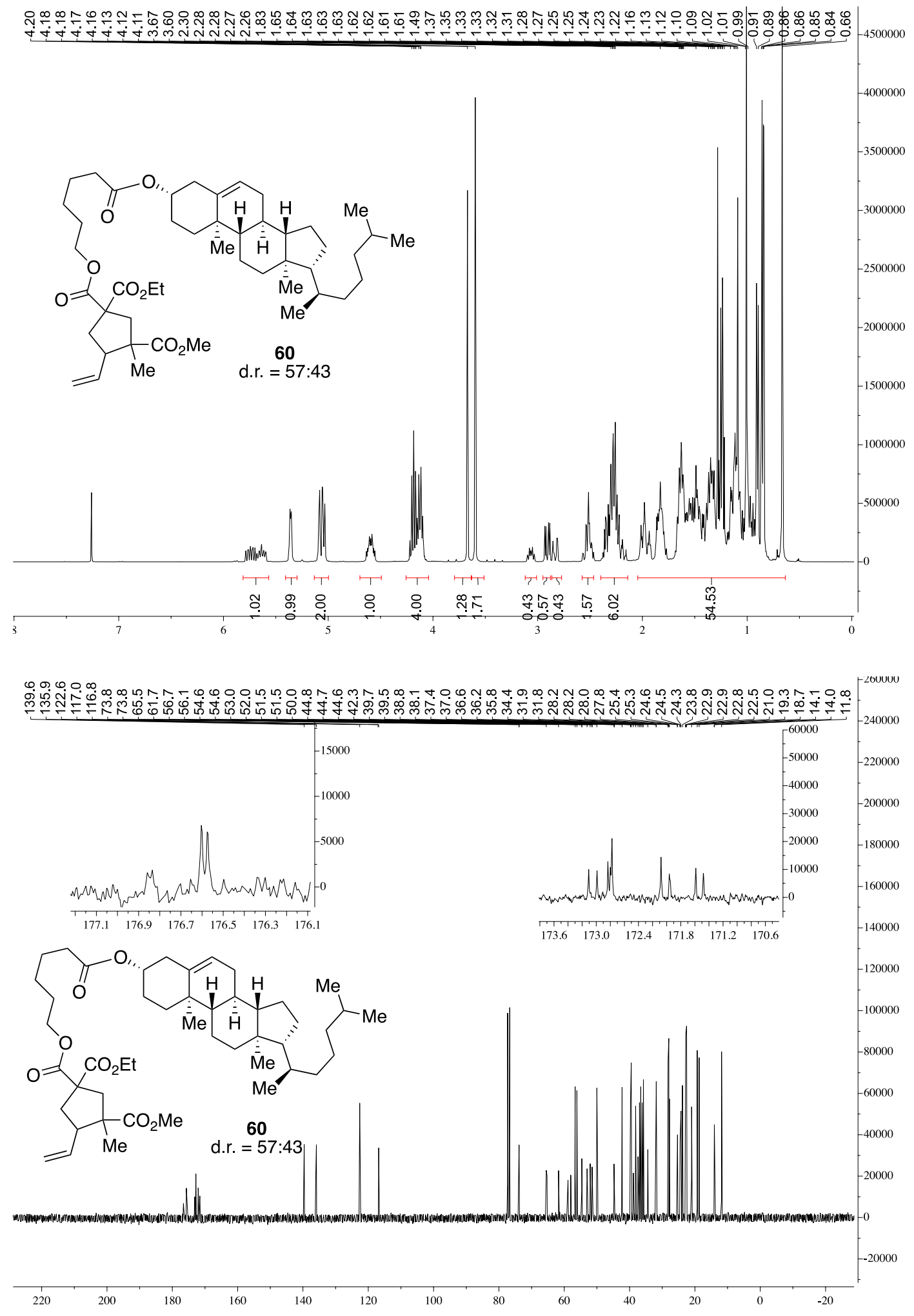



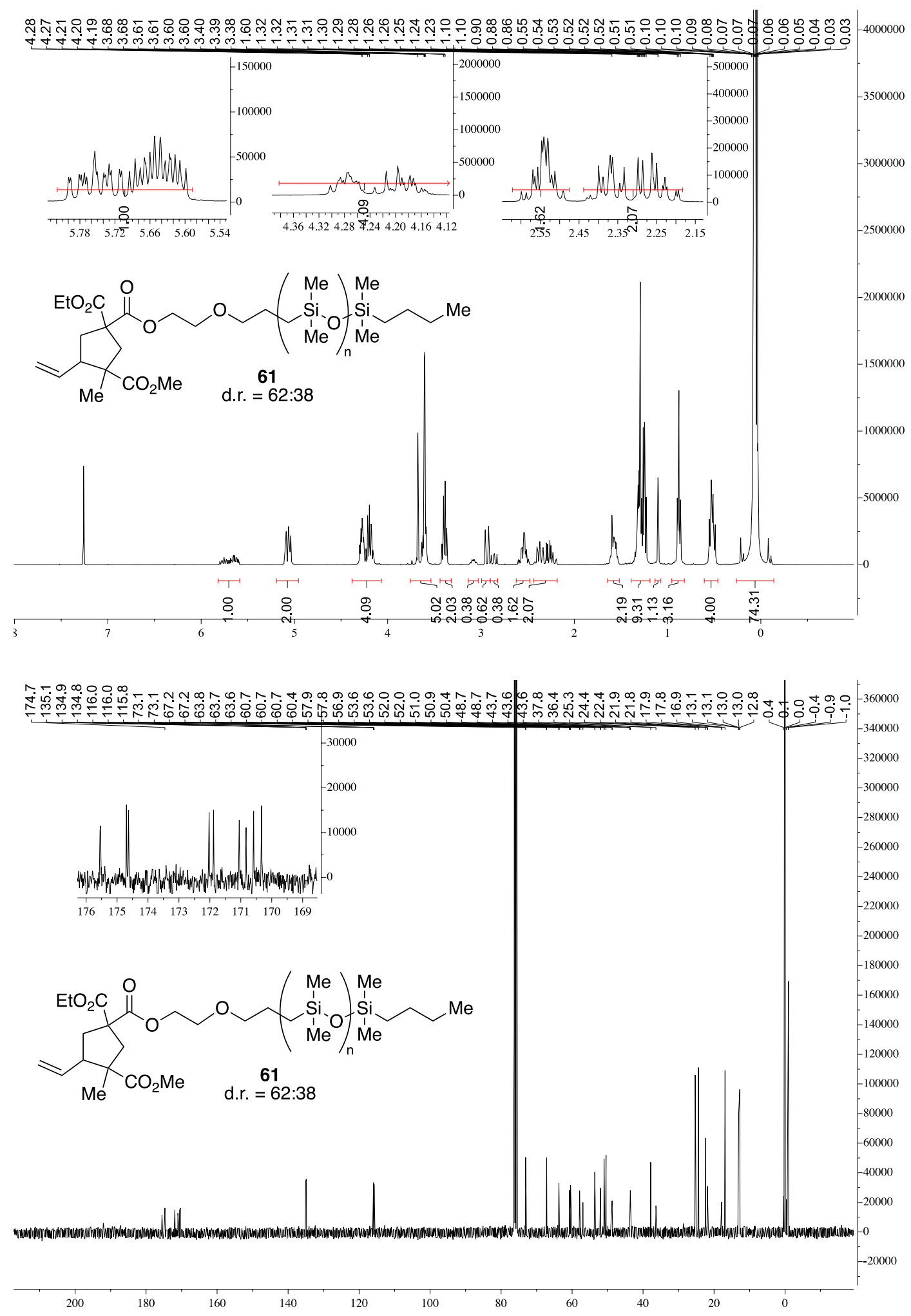

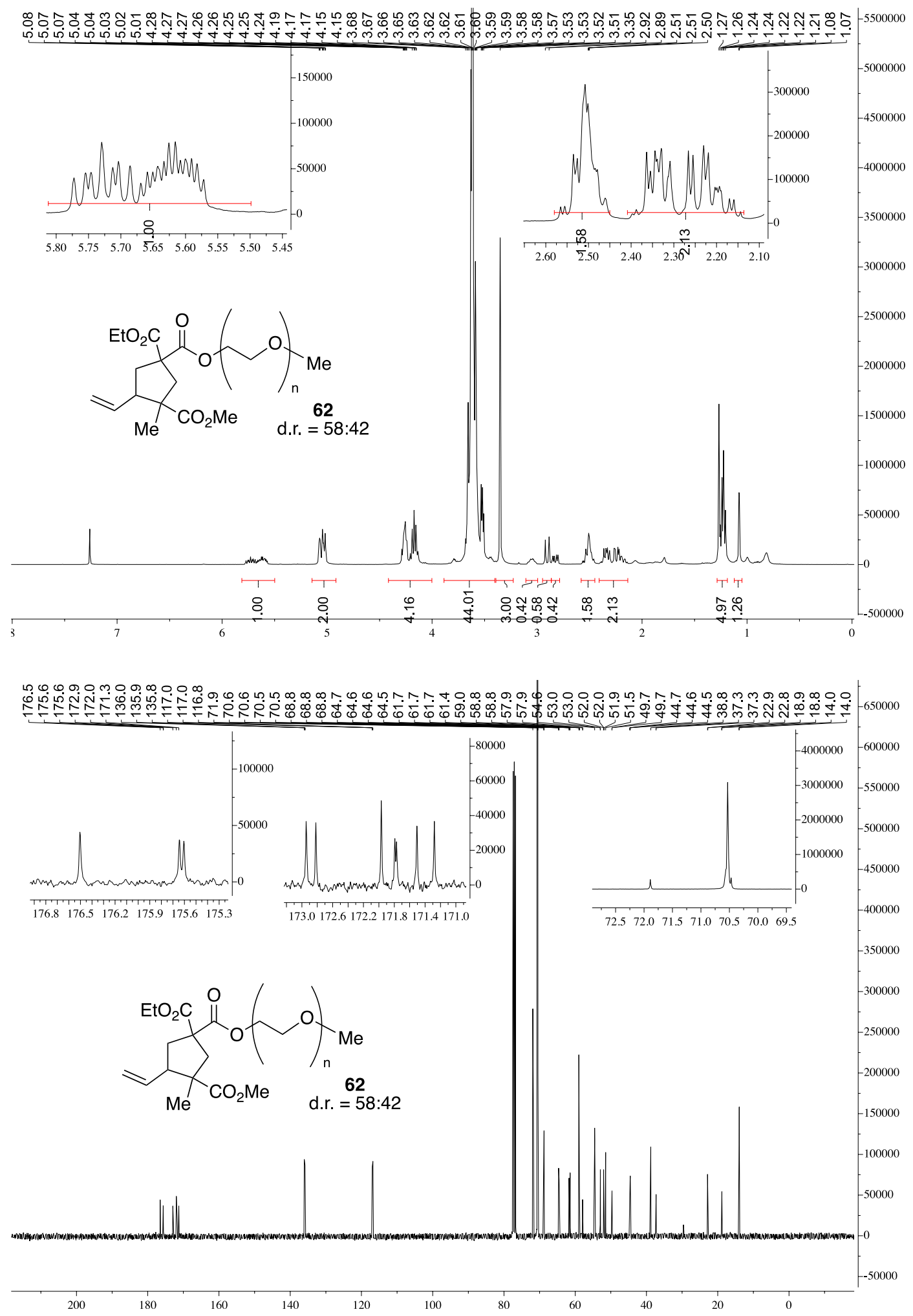


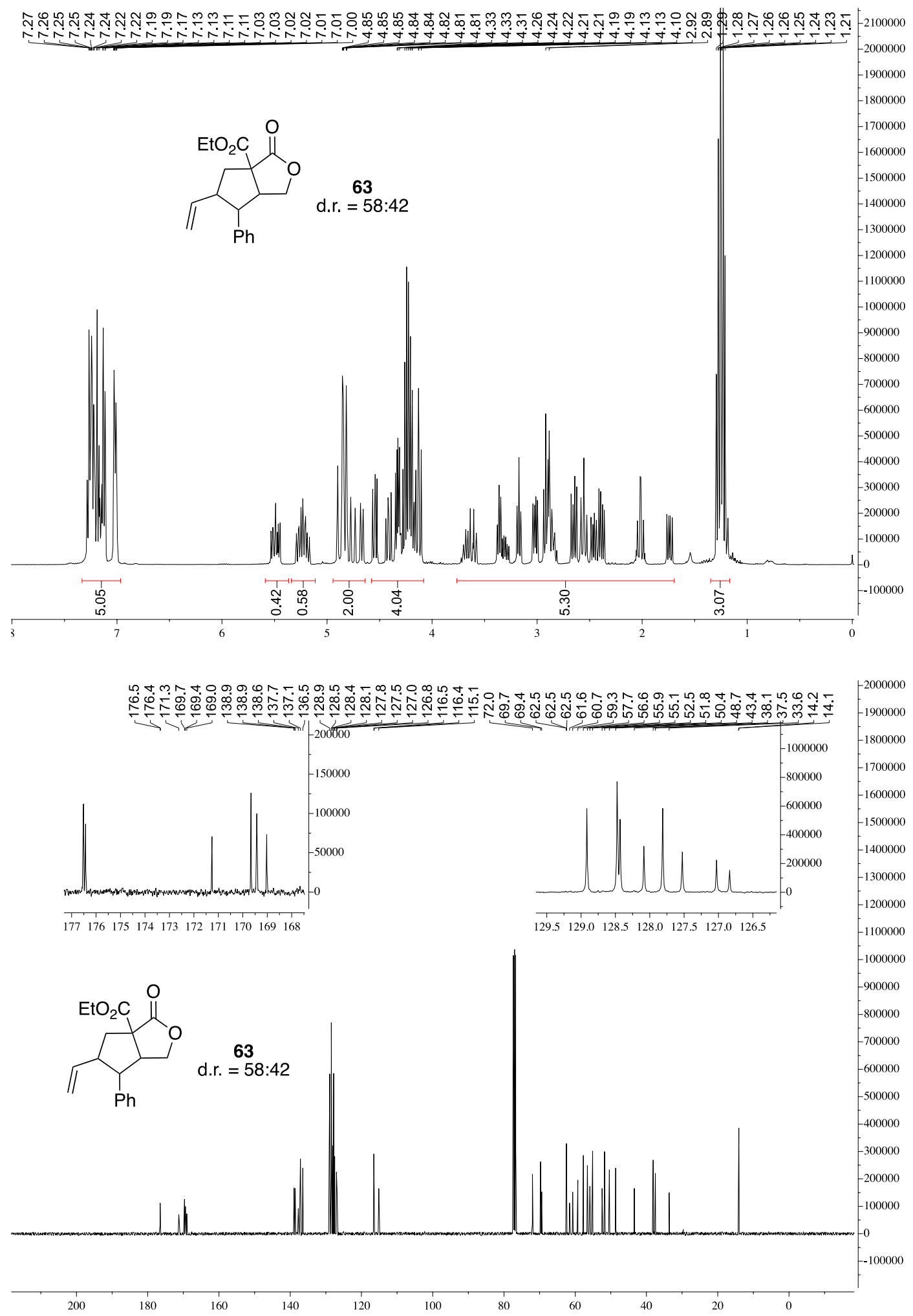



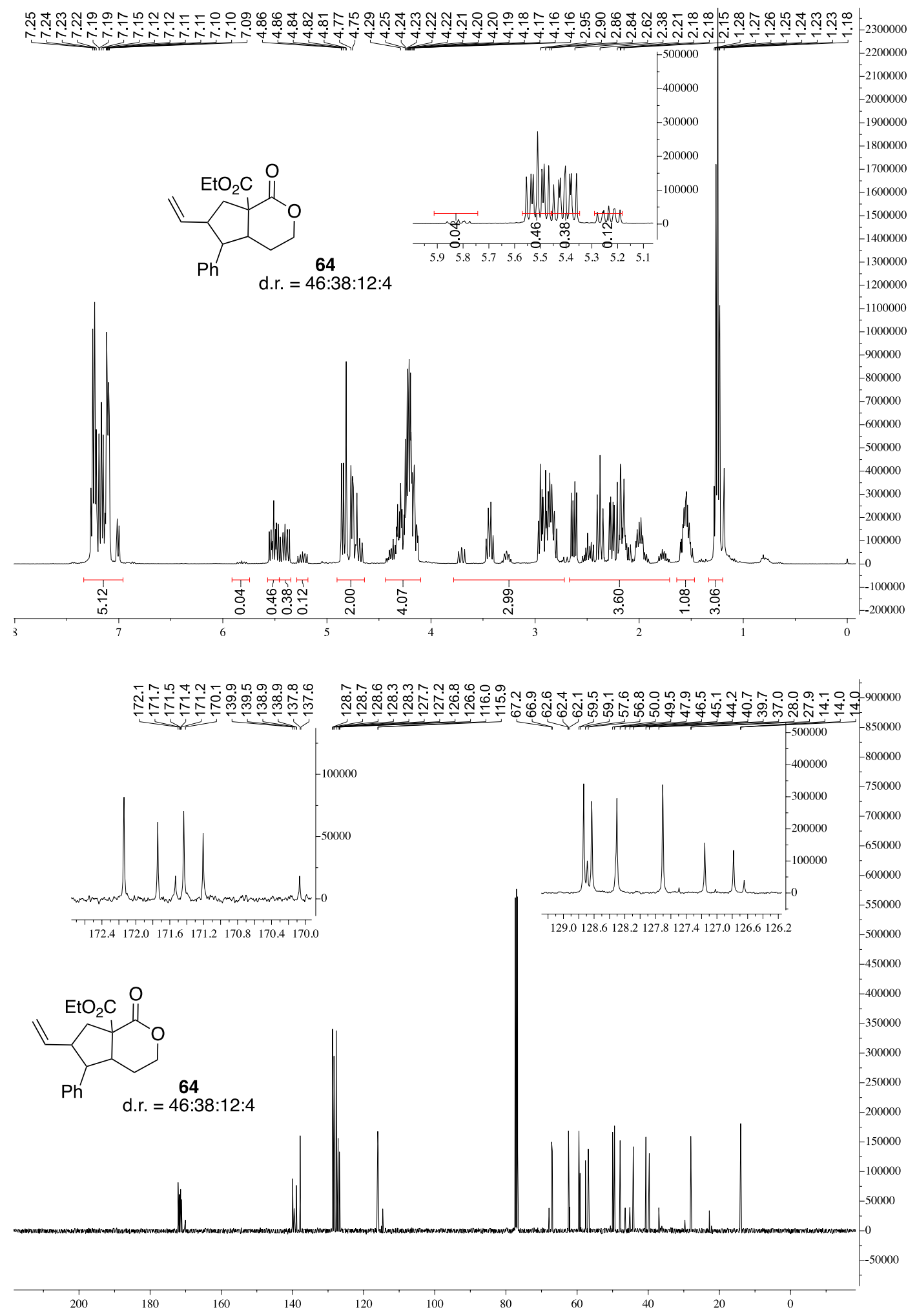


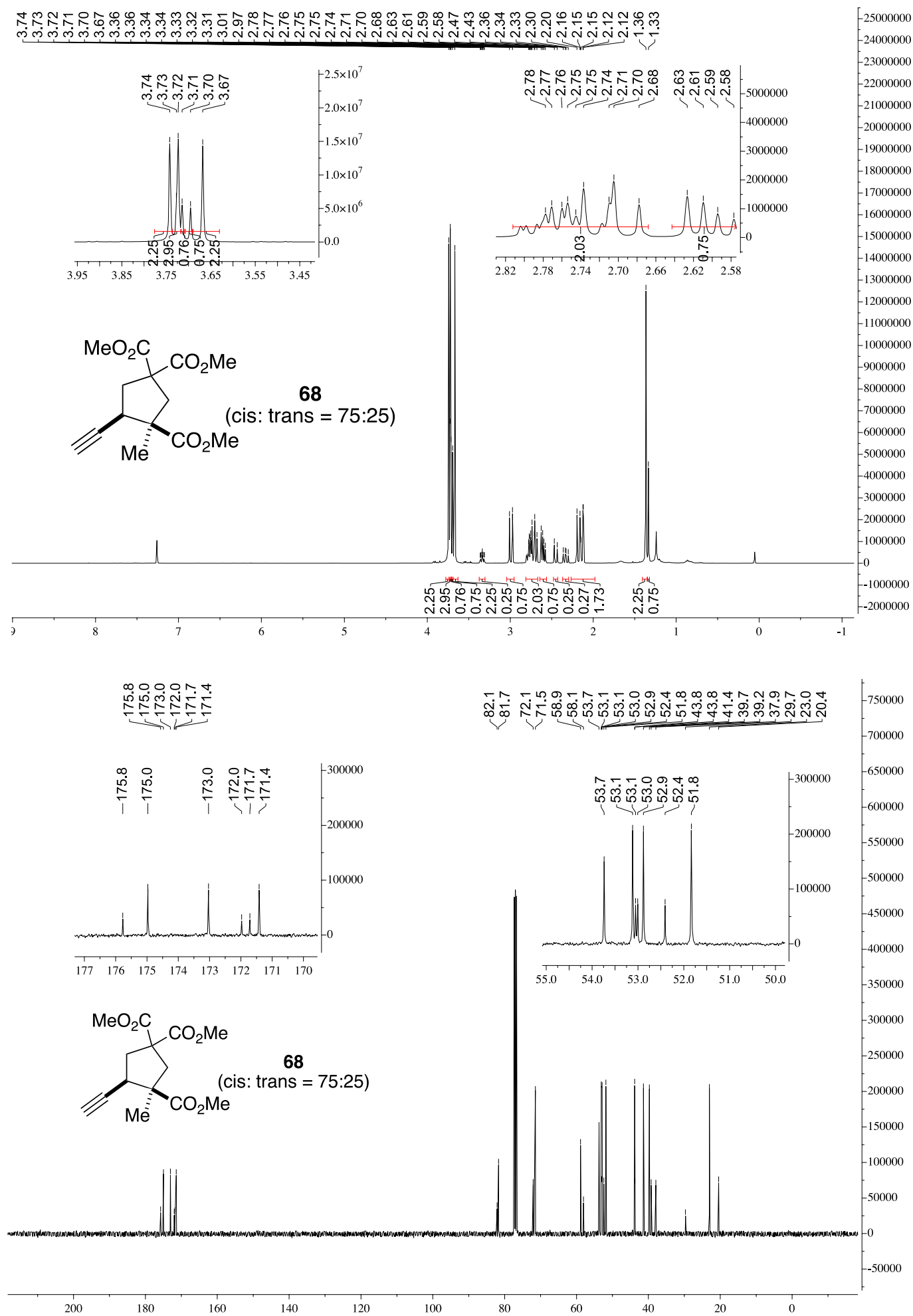




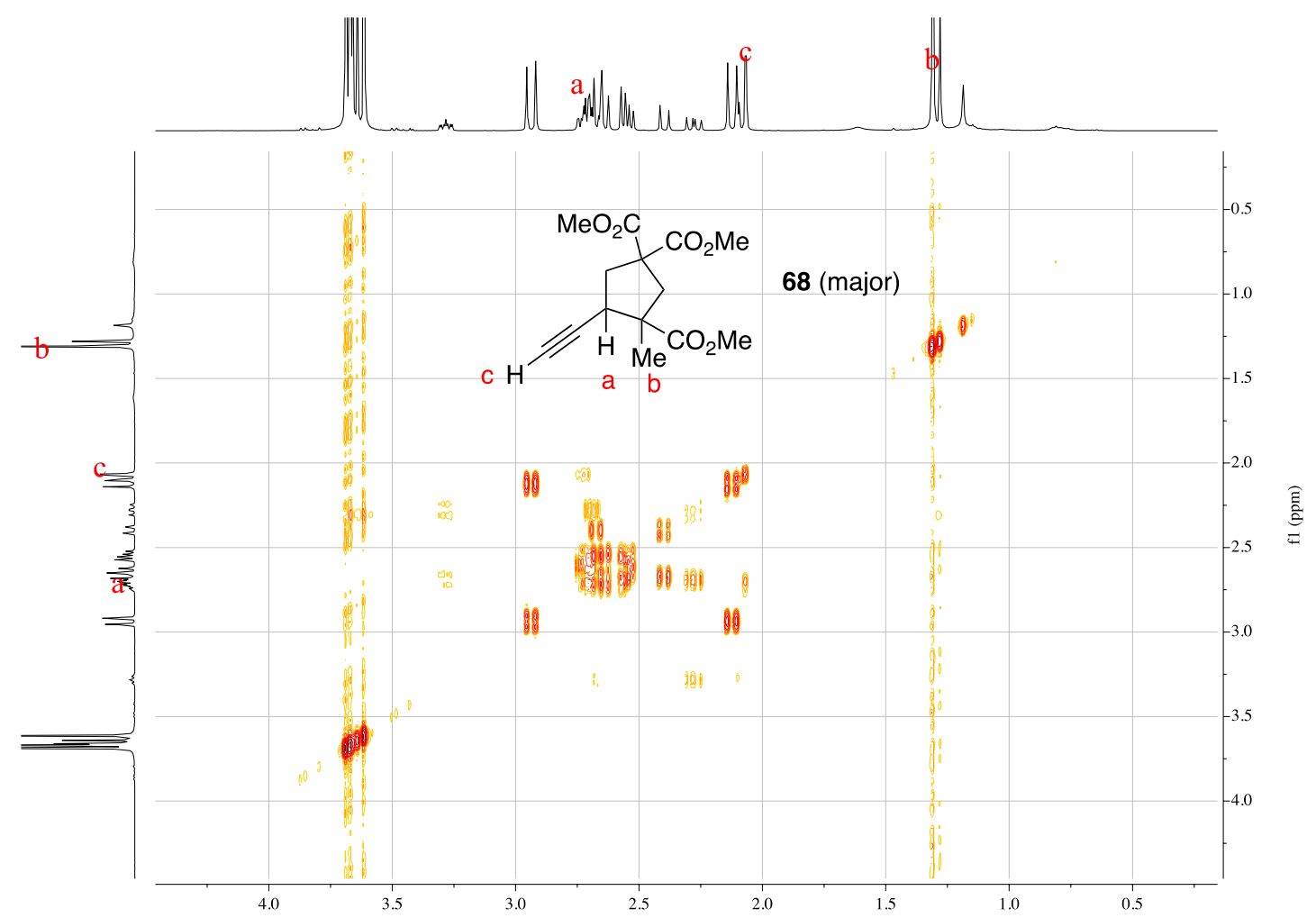




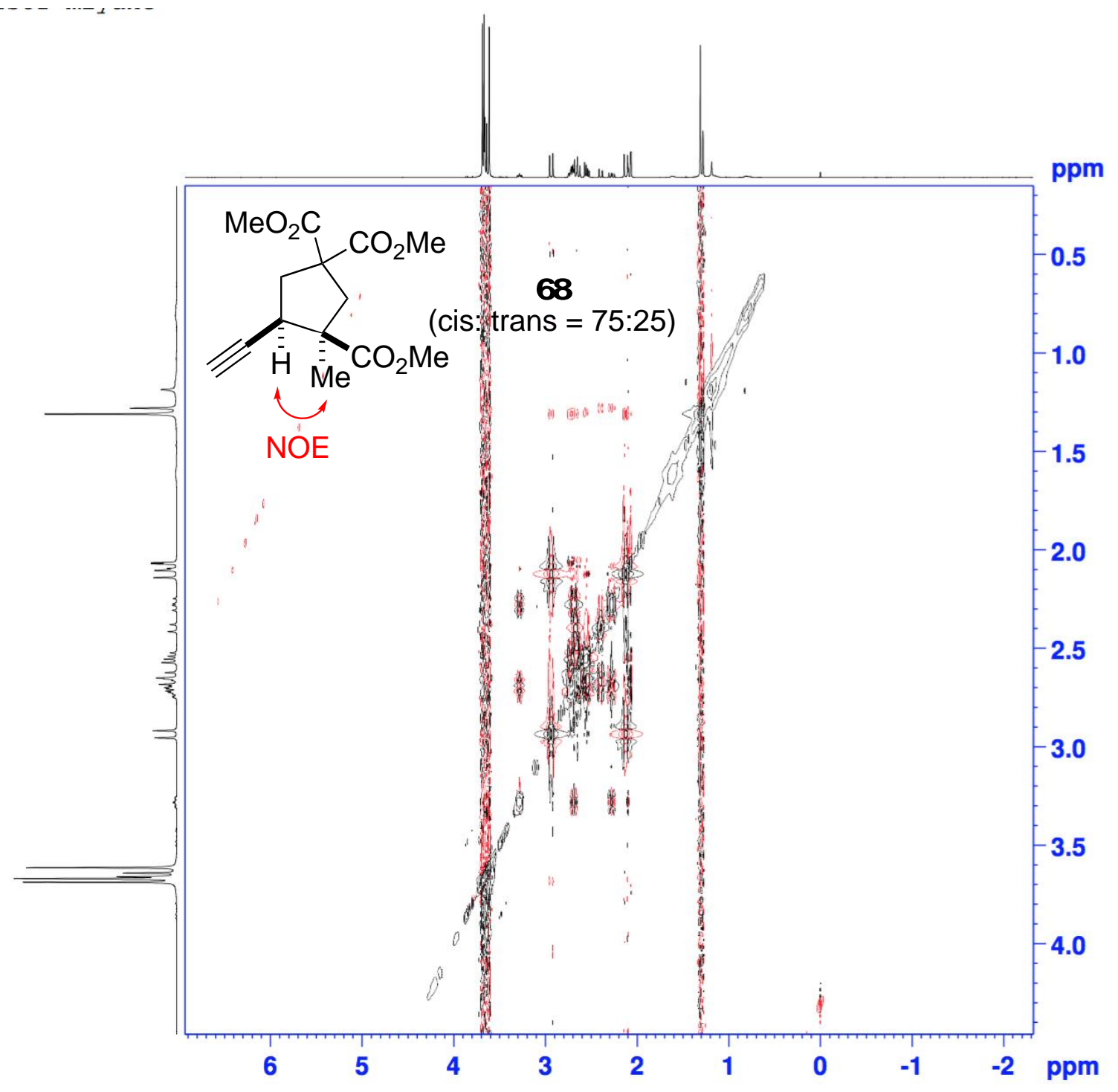



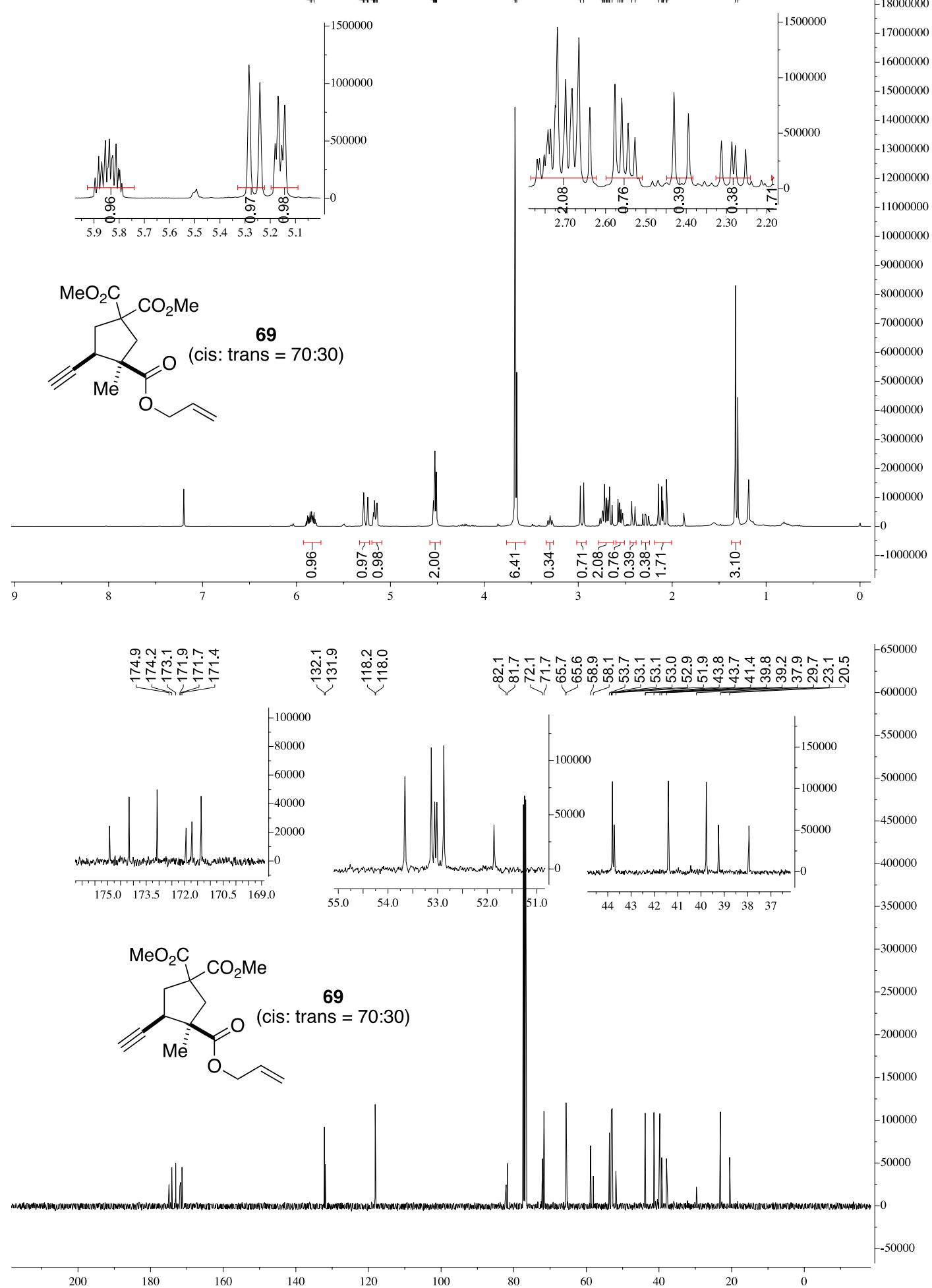